\title{
Stochastic Gravity: Theory and Applications
}

\author{
Bei Lok $\mathrm{Hu}$ \\ Department of Physics \\ University of Maryland \\ College Park, Maryland 20742-4111 \\ U.S.A. \\ email: hub@physics.umd.edu \\ http://www .physics . umd.edu/people/faculty/hu.html \\ Enric Verdaguer \\ Departament de Física Fonamental and \\ Institut de Ciències del Cosmos \\ Universitat de Barcelona \\ Av. Diagonal 647, 08028 Barcelona \\ Spain \\ email: enric.verdaguer@ub.edu
}

\section{Living Reviews in Relativity ISSN 1433-8351}

Accepted on 11 April 2008

Published on 29 May 2008

\begin{abstract}
Whereas semiclassical gravity is based on the semiclassical Einstein equation with sources given by the expectation value of the stress-energy tensor of quantum fields, stochastic semiclassical gravity is based on the Einstein-Langevin equation, which has, in addition, sources due to the noise kernel. The noise kernel is the vacuum expectation value of the (operatorvalued) stress-energy bitensor, which describes the fluctuations of quantum-matter fields in curved spacetimes. A new improved criterion for the validity of semiclassical gravity may also be formulated from the viewpoint of this theory. In the first part of this review we describe the fundamentals of this new theory via two approaches: the axiomatic and the functional. The axiomatic approach is useful to see the structure of the theory from the framework of semiclassical gravity, showing the link from the mean value of the stress-energy tensor to the correlation functions. The functional approach uses the Feynman-Vernon influence functional and the Schwinger-Keldysh closed-time-path effective action methods. In the second part, we describe three applications of stochastic gravity. First, we consider metric perturbations in a Minkowski spacetime, compute the two-point correlation functions of these perturbations and prove that Minkowski spacetime is a stable solution of semiclassical gravity. Second, we discuss structure formation from the stochastic-gravity viewpoint, which can go beyond the standard treatment by incorporating the full quantum effect of the inflaton fluctuations. Third, using the Einstein-Langevin equation, we discuss the backreaction of Hawking radiation and the behavior of metric fluctuations for both the quasi-equilibrium condition of a black-hole in a box and the fully nonequilibrium condition of an evaporating black hole spacetime. Finally, we briefly discuss the theoretical structure of stochastic gravity in relation to quantum gravity and point out directions for further developments and applications.
\end{abstract}

This review is licensed under a Creative Commons Attribution-Non-Commercial-NoDerivs 2.0 Germany License. http://creativecommons.org/licenses/by-nc-nd/2.0/de/ 


\section{Imprint / Terms of Use}

Living Reviews in Relativity is a peer reviewed open access journal published by the Max Planck Institute for Gravitational Physics, Am Mühlenberg 1, 14476 Potsdam, Germany. ISSN 1433-8351.

This review is licensed under a Creative Commons Attribution-Non-Commercial-NoDerivs 2.0 Germany License: http://creativecommons.org/licenses/by-nc-nd/2.0/de/

Because a Living Reviews article can evolve over time, we recommend to cite the article as follows:

Bei Lok Hu and Enric Verdaguer,

"Stochastic Gravity: Theory and Applications",

Living Rev. Relativity, 11, (2008), 3. [Online Article]: cited [<date $>$,

http://www.livingreviews.org/lrr-2008-3

The date given as $<$ date $>$ then uniquely identifies the version of the article you are referring to.

\section{Article Revisions}

Living Reviews supports two different ways to keep its articles up-to-date:

Fast-track revision A fast-track revision provides the author with the opportunity to add short notices of current research results, trends and developments, or important publications to the article. A fast-track revision is refereed by the responsible subject editor. If an article has undergone a fast-track revision, a summary of changes will be listed here.

Major update A major update will include substantial changes and additions and is subject to full external refereeing. It is published with a new publication number.

For detailed documentation of an article's evolution, please refer always to the history document of the article's online version at http://www.livingreviews.org/lrr-2008-3. 


\section{Contents}

1 Overview $\quad 5$

2 From Semiclassical to Stochastic Gravity $\quad \mathbf{8}$

2.1 The importance of quantum fluctuations . . . . . . . . . . . . . . . 8

3 The Einstein-Langevin Equation: Axiomatic Approach 11

3.1 Semiclassical gravity . . . . . . . . . . . . . . . . . . . . 11

3.2 Stochastic gravity . . . . . . . . . . . . . . . . . . . . 13

3.3 Validity of semiclassical gravity . . . . . . . . . . . . . . . . . . 17

3.3 .1 The large $\mathrm{N}$ expansion . . . . . . . . . . . . . . . . 18

4 The Einstein-Langevin Equation: Functional Approach $\quad 21$

4.1 Influence action for semiclassical gravity . . . . . . . . . . . . . . . . . . . 21

4.2 Influence action for stochastic gravity . . . . . . . . . . . . . . . . . . 23

4.3 Explicit form of the Einstein-Langevin equation . . . . . . . . . . . . . . . . . 25

4.3.1 The kernels for the vacuum state . . . . . . . . . . . . . 26

5 Noise Kernel and Point Separation $\quad 28$

5.1 Point separation . . . . . . . . . . . . . . . . . . . 29

$5.1 .1 n$-tensors and end-point expansions . . . . . . . . . . . . . . . . 29

5.2 Stress-energy bitensor operator and noise kernel . . . . . . . . . . . . . . . . 31

5.2 .1 Finiteness of the noise kernel . . . . . . . . . . . . . . . . . . 32

5.2 .2 Explicit form of the noise kernel . . . . . . . . . . . . . . . 33

5.2 .3 Trace of the noise kernel . . . . . . . . . . . . . . . . . 35

6 Metric Fluctuations in Minkowski Spacetime $\quad 37$

6.1 Perturbations around Minkowski spacetime . . . . . . . . . . . . . . 37

6.2 The kernels in the Minkowski background . . . . . . . . . . . . . . . . . . . 39

6.3 The Einstein-Langevin equation . . . . . . . . . . . . . . . . . . . . . . 42

6.4 Correlation functions for gravitational perturbations . . . . . . . . . . . . . . . . 44

6.4.1 Correlation functions for the linearized Einstein tensor . . . . . . . . . . 45

6.4.2 Correlation functions for the metric perturbations . . . . . . . . . . 47

6.4.3 Conformally-coupled field . . . . . . . . . . . . . . . . 48

6.5 Stability of Minkowski spacetime . . . . . . . . . . . . . . . . . . 49

6.5.1 Intrinsic metric fluctuations . . . . . . . . . . . . . . . . . . . 50

6.5 .2 Induced metric fluctuations . . . . . . . . . . . . . . . . . . 52

6.5.3 Order-reduction prescription and large $\mathrm{N} \ldots \ldots \ldots 54$

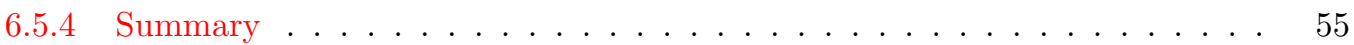

7 Structure Formation in the Early Universe $\quad \mathbf{5 7}$

7.1 The model . . . . . . . . . . . . . . . . . . . . . 57

7.2 Einstein-Langevin equation for scalar metric perturbations . . . . . . . . . . 58

7.3 Correlation functions for scalar metric perturbations . . . . . . . . . . . . . 59

7.4 Summary and outlook . . . . . . . . . . . . . . . . . 61 
8 Black Hole Backreaction and Fluctuations $\quad 63$

8.1 General issues of backreaction . . . . . . . . . . . . . . . . . . . 63

8.1.1 Regularized energy-momentum tensor . . . . . . . . . . . . . 63

8.1.2 Backreaction and fluctuation-dissipation relation . . . . . . . . . . . 64

8.1.3 Noise and fluctuations - the missing ingredient in older treatments . . . . . 65

8.2 Backreaction on black holes under quasi-static conditions . . . . . . . . . . . . 65

8.2 .1 The model . . . . . . . . . . . . . . . . 66

8.2.2 CTP effective action for the black hole . . . . . . . . . . . . . . 67

8.2 .3 Near flat case . . . . . . . . . . . . . . . . . . . . . . . . . . 69

8.2 .4 Near-horizon case . . . . . . . . . . . . . . . . . . . . 71

8.2.5 Einstein-Langevin equation . . . . . . . . . . . . . . . . . 72

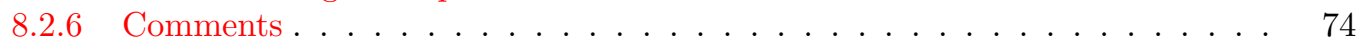

8.3 Metric fluctuations of an evaporating black hole . . . . . . . . . . . . . . . . . 75

8.3.1 Evolution of the mean geometry of an evaporating black hole . . . . . . . 75

8.3.2 Spherically-symmetric induced fluctuations . . . . . . . . . . . . . . 77

8.3 .3 Summary and prospects . . . . . . . . . . . . . . . . . . 81

8.4 Other work on metric fluctuations but without backreaction . . . . . . . . . . . 82

9 Concluding Remarks $\quad 83$

10 Acknowledgements $\quad 84$

$\begin{array}{lr}\text { References } & 85\end{array}$ 


\section{Overview}

Stochastic semiclassical gravity is a theory developed in the 1990s using semiclassical gravity (quantum fields in classical spacetimes, the dynamics of both matter and spacetime are solved self-consistently) as the starting point and aiming at a theory of quantum gravity as the goal. While semiclassical gravity is based on the semiclassical Einstein equation with the source given by the expectation value of the stress-energy tensor of quantum fields, stochastic semiclassical gravity, or stochastic gravity for short, also includes its fluctuations in a new stochastic semiclassical Einstein-Langevin equation (we will often use the shortened term stochastic gravity as there is no confusion as to the nature and source of stochasticity in gravity being induced from the quantum fields and not a priori from the classical spacetime). If the centerpiece in semiclassicalgravity theory is the vacuum expectation value of the stress-energy tensor of a quantum field and the central issues are how well the vacuum is defined and how the divergences can be controlled by regularization and renormalization, the centerpiece in stochastic semiclassical-gravity theory is the stress-energy bitensor and its expectation value known as the noise kernel. The mathematical properties of this quantity and its physical content in relation to the behavior of fluctuations of quantum fields in curved spacetimes are the central issues of this new theory. How they induce metric fluctuations and seed the structures of the universe, how they affect the black-hole horizons and the backreaction of Hawking radiance in black hole dynamics, including implications for trans-Planckian physics, are new horizons to explore. On theoretical issues, stochastic gravity is the necessary foundation to investigate the validity of semiclassical gravity and the viability of inflationary cosmology based on the appearance and sustenance of a vacuum energy-dominated phase. It is also a useful beachhead supported by well-established low-energy (sub-Planckian) physics from which to explore the connection with high-energy (Planckian) physics in the realm of quantum gravity.

In this review we summarize the major work and results of this theory since 1998. It is in the nature of a progress report rather than a review. In fact we will have room only to discuss a handful of topics of basic importance. A review of ideas leading to stochastic gravity and further developments originating from it can be found in [181, 187], a set of lectures, which include a discussion of the issue of the validity of semiclassical gravity in [207] and a pedagogical introduction of stochastic-gravity theory with a more detailed treatment of backreaction problems in cosmology and black holes in quasi-equilibrium in [208]. A comprehensive formal description of the fundamentals is given in [257, 258], while that of the noise kernel in arbitrary spacetimes can be found in $[258,304,305]$. Here we will try to mention related work so the reader can at least trace out the parallel and sequential developments. The references at the end of each topic below are representative work in which one can seek out further treatments.

Stochastic gravity theory is built on three pillars: general relativity, quantum fields and nonequilibrium statistical mechanics. The first two uphold semiclassical gravity, the last two span statistical field theory. Strictly speaking one can understand a great deal without appealing to statistical mechanics, and we will try to do so here. But concepts such as quantum open systems [88, 246, 370] and techniques such as the influence functional $[107,108]$ (which is related to the closed-time-path effective action $[14,54,56,82,87,94,222,223,227,296,323,343])$ were a great help in our understanding of the physical meaning of issues involved in the construction of this new theory. Foremost because quantum fluctuations and correlation have ascended the stage and become the focus of attention. Quantum statistical field theory and the statistical mechanics of quantum field theory $[55,57,59,61]$ also aided us in searching for the connection with quantum gravity through the retrieval of correlations and coherence.

We show the scope of stochastic gravity as follows: 


\section{Ingredients:}

(a) From General Relativity [266, 361] to Semiclassical Gravity.

(b) Quantum Field Theory in Curved Spacetimes [34, 121, 135, 362]:

i. Stress-energy tensor: Regularization and renormalization.

ii. Self-consistent solution: Backreaction problems in early universe and black holes [3, $4,5,109,137,147,148,153,154,165,193,194,251]$, and analog gravity [15, 16, $252,320,321]$.

iii. Effective action: Closed time path, initial value formulation $[14,54,56,82,87,94$, 223, 227, 296, 323, 343].

iv. Equation of motion: Real and causal [222].

(c) Nonequilibrium Statistical Mechanics (see [62] and references therein) :

i. Open quantum systems [88, 246, 370].

ii. Influence Functional: Stochastic equations [107, 108].

iii. Noise and Decoherence: Quantum to classical transition [43, 46, 99, 100, 101, 126, 131, 136, 144, 145, 146, 149, 209, 210, 211, 212, 221, 228, 229, 230, 275, 276, 277, 278, 279, 280, 299, 350, 352, 389, 390, 391, 392, 393].

(d) Decoherence in Quantum Cosmology and Emergence of Classical Spacetimes [50, 51, 143, 182, 195, 231, 283].

\section{Theory:}

(a) Dissipation from Particle Creation [54, 72, 94, 222, 223, 296]; Backreaction as Fluctuation-Dissipation Relation (FDR) [69, 76, 206, 268].

(b) Noise from Fluctuations of Quantum Fields [58, 181, 183].

(c) Einstein-Langevin Equations [52, 58, 73, 74, 192, 206, 248, 256, 257, 258].

(d) Metric Fluctuations in Minkowski spacetime [259].

\section{Issues:}

(a) Validity of Semiclassical Gravity [10, 111, 169, 170, 198, 203, 204, 223, 237, 303, 329].

(b) Viability of Vacuum Dominance and Inflationary Cosmology.

(c) Stress-Energy Bitensor and Noise Kernel: Regularization Reassessed [304, 305].

\section{Applications: Early Universe and Black Holes:}

(a) Wave Propagation in Stochastic Geometry [205].

(b) Black Hole Horizon Fluctuations: Spontaneous/Active versus Induced/Passive [20, 21, 114, 261, 289, 305, 336, 338, 377].

(c) Noise induced inflation [67].

(d) Structure Formation [53, 60, 262, 263, 316]; trace anomaly-driven inflation [162, 339, 355].

(e) Black Hole Backreaction and Fluctuations [69, 70, 76, 199, 200, 201, 268, 324, 325, 332].

\section{Related Topics:}

(a) Metric Fluctuations and Trans-Planckian Problem [20, 21, 261, 273, 289].

(b) Spacetime Foam, Loop and Spin Foam [32, 78, 79, 85, 116, 122, 123, 124, 271, 272]. 
(c) Universal 'Metric Conductance' Fluctuations [328].

\section{Ideas:}

(a) General Relativity as Geometro-Hydrodynamics [105, 164, 185, 189, 216, 356, 357]; Emergent Gravity [139, 173, 240, 326].

(b) Semiclassical Gravity as Mesoscopic Physics [186, 190].

(c) From Stochastic to Quantum Gravity:

i. Via Correlation hierarchy of interacting quantum fields [57, 61, 187, 188].

ii. Possible relation to string theory and matrix theory.

iii. Other major approaches to quantum gravity [281].

For lack of space we list only the latest work in the respective topics above, describing ongoing research. The reader should consult the references therein for earlier work and background material. We do not seek a complete coverage here, but will discuss only those selected topics in theory, issues and applications. We use the $(+,+,+)$ sign conventions of [266,361], and units in which $c=\hbar=1$. 


\section{From Semiclassical to Stochastic Gravity}

There are three main steps that led to the recent development of stochastic gravity. The first step begins with quantum field theory in curved spacetime [34, 93, 121, 135, 362], which describes the behavior of quantum matter fields propagating in a specified (not dynamically determined by the quantum matter field as source) background gravitational field. In this theory the gravitational field is given by the classical spacetime metric determined from classical sources by the classical Einstein equations and the quantum fields propagate as test fields in such a spacetime. Some important processes described by quantum field theory in curved spacetime are particle creation from the vacuum, effects of vacuum fluctuations and polarizations in the early universe [29, 30, 31, 81, 93, 117, 179, 293, 327, 386, 387], and Hawking radiation in black holes [155, 156, 213, 294, 358].

The second step in the description of the interaction of gravity with quantum fields is backreaction, i.e., the effect of quantum fields on spacetime geometry. The source here is the expectation value of the stress-energy operator for matter fields in some quantum state in the spacetime, a classical observable. However, since this object is quadratic in the field operators, which are only well-defined as distributions on the spacetime, it involves ill-defined quantities. It contains ultraviolet divergences, the removal of which requires a renormalization procedure [83, 84, 93]. The final expectation value of the stress-energy operator using a reasonable regularization technique is essentially unique, modulo some terms, which depend on the spacetime curvature and which are independent of the quantum state. This uniqueness was proved by Wald [359, 360] who investigated the criteria that a physically meaningful expectation value of the stress-energy tensor ought to satisfy.

The theory obtained from a self-consistent solution of the geometry of the spacetime and the quantum field is known as semiclassical gravity. Incorporating the backreaction of the quantum matter field into the spacetime is thus the central task in semiclassical gravity. One assumes a general class of spacetime, in which the quantum fields live and on which they act and seek a solution that satisfies simultaneously the Einstein equation for the spacetime and the field equations for the quantum fields. The Einstein equation, which has the expectation value of the stress-energy operator of the quantum matter field as its source, is known as the semiclassical Einstein equation. Semiclassical gravity was first investigated in cosmological backreaction problems [3, 4, 109, 137, $147,148,153,154,193,194,251]$; an example is the damping of anisotropy in Bianchi universes by the backreaction of vacuum particle creation. The effect of quantum field processes, such as particle creation, was used to explain why the universe is so isotropic at the present in the context of chaotic cosmology [27, 28, 265] in the late 1970s prior to the inflationary-cosmology proposal of the 1980s [2, 140, 243, 244], which assumes the vacuum expectation value of an inflaton field as the source, another, perhaps more well-known, example of semiclassical gravity.

\subsection{The importance of quantum fluctuations}

For a free quantum field, semiclassical gravity is fairly well understood. The theory is in some sense unique, since the only reasonable c-number stress-energy tensor that one may construct [359, 360] with the stress-energy operator is a renormalized expectation value. However, the scope and limitations of the theory are not so well understood. It is expected that the semiclassical theory will break down at the Planck scale. One can conceivably assume that it will also break down when the fluctuations of the stress-energy operator are large [111, 237]. Calculations of the fluctuations of the energy density for Minkowski, Casimir and hot flat spaces as well as Einstein and de Sitter universes are available [86, 198, 237, 258, 259, 282, 302, 303, 304, 305, 313, 316]. It is less clear, however, how to quantify what a large fluctuation is, and different criteria have been proposed [10,11, 113, 115, 198, 237, 303, 384, 385]. The issue of the validity of semiclassical gravity viewed in the light of quantum fluctuations was discussed in our Erice lectures [207]. More 
recently in [203, 204] a new criterion has been proposed for the validity of semiclassical gravity. It is based on quantum fluctuations of the semiclassical metric and incorporates, in a unified and self-consistent way, previous criteria that have been used [10, 111, 169, 237]. One can see the essence of the validity problem in the following example inspired by Ford [111].

Let us assume a quantum state formed by an isolated system, which consists of a superposition with equal amplitude of one configuration of mass $M$ with the center of mass at $X_{1}$ and another configuration of the same mass with the center of mass at $X_{2}$. The semiclassical theory, as described by the semiclassical Einstein equation, predicts that the center of mass of the gravitational field of the system is centered at $\left(X_{1}+X_{2}\right) / 2$. However, one would expect that if we send a succession of test particles to probe the gravitational field of the above system, half of the time they would react to a gravitational field of mass $M$ centered at $X_{1}$ and half of the time to the field centered at $X_{2}$. The two predictions are clearly different; note that the fluctuation in the position of the center of masses is on the order of $\left(X_{1}-X_{2}\right)^{2}$. Although this example raises the issue of the importance of fluctuations to the mean, a word of caution should be added to the effect that it should not be taken too literally. In fact, if the previous masses are macroscopic, the quantum system decoheres very quickly [392, 393] and, instead of being described by a pure quantum state, it is described by a density matrix, which diagonalizes in a certain pointer basis. For observables associated with such a pointer basis, the density matrix description is equivalent to that provided by a statistical ensemble. The results will differ, in any case, from the semiclassical prediction.

In other words, one would expect that a stochastic source that describes the quantum fluctuations should enter into the semiclassical equations. A significant step in this direction was made in [181] where it was proposed that one view the backreaction problem in the framework of an open quantum system: the quantum fields acting as the "environment" and the gravitational field as the "system". Following this proposal a systematic study of the connection between semiclassical gravity and open quantum systems resulted in the development of a new conceptual and technical framework in which (semiclassical) Einstein-Langevin equations were derived $[52,58,73,74,192,206,248]$. The key technical factor to most of these results was the use of the influence-functional method of Feynman and Vernon [108], when only the coarse-grained effect of the environment on the system is of interest. Note that the word semiclassical put in parentheses refers to the fact that the noise source in the Einstein-Langevin equation arises from the quantum field, while the background spacetime is classical; generally we will not carry this word since there is no confusion that the source, which contributes to the stochastic features of this theory, comes from quantum fields.

In the language of the consistent-histories formulation of quantum mechanics [43, 99, 100, 101, 126, 136, 144, 145, 146, 149, 209, 210, 211, 212, 228, 229, 230, 275, 276, 277, 278, 279, 280, 299, 350], for the existence of a semiclassical regime for the dynamics of the system, one has two requirements. The first is decoherence, which guarantees that probabilities can be consistently assigned to histories describing the evolution of the system, and the second is that these probabilities should peak near histories, which correspond to solutions of classical equations of motion. The effect of the environment is crucial, on the one hand, to provide decoherence and, on the other hand, to produce both dissipation and noise in the system through backreaction, thus inducing a semiclassical stochastic dynamic in the system. As shown by different authors [46, 127, 131, 221, 352, 389, 390, 391, 392, 393], indeed over a long history predating the current revival of decoherence, stochastic semiclassical equations are obtained in an open quantum system after a coarse-graining of the environmental degrees of freedom and a further coarse-graining in the system variables. It is expected, but has not yet been shown, that this mechanism could also work for decoherence and classicalization of the metric field. Thus far, the analogy could only be made formally [256] or under certain assumptions, such as adopting the Born-Oppenheimer approximation in quantum cosmology [297, 298].

An alternative axiomatic approach to the Einstein-Langevin equation, without invoking the 
open-system paradigm, was later suggested based on the formulation of a self-consistent dynamical equation for a perturbative extension of semiclassical gravity able to account for the lowest-order stress-energy fluctuations of matter fields [257]. It was shown that the same equation could be derived, in this general case, from the influence functional of Feynman and Vernon [258]. The field equation is deduced via an effective action, which is computed assuming that the gravitational field is a c-number. The important new element in the derivation of the Einstein-Langevin equation, and of stochastic-gravity theory, is the physical observable that measures the stress-energy fluctuations, namely, the expectation value of the symmetrized bitensor constructed with the stress-energy tensor operator: the noise kernel. It is interesting to note that the Einstein-Langevin equation can also be understood as a useful intermediary tool to compute symmetrized two-point correlations of the quantum metric perturbations on the semiclassical background, independent of a suitable classicalization mechanism [317]. 


\section{The Einstein-Langevin Equation: Axiomatic Approach}

In this section we introduce stochastic semiclassical gravity, or stochastic gravity for short, in an axiomatic way. It is introduced as an extension of semiclassical gravity motivated by the search for self-consistent equations, which describe the backreaction of the quantum stress-energy fluctuations on the gravitational field [257].

\subsection{Semiclassical gravity}

Semiclassical gravity describes the interaction of a classical gravitational field with quantum matter fields. This theory can be formally derived as the leading $1 / N$ approximation of quantum gravity interacting with $N$ independent and identical free quantum fields [152, 175, 176, 348], which interact with gravity only. By keeping the value of $N G$ finite, where $G$ is Newton's gravitational constant, one arrives at a theory in which formally the gravitational field can be treated as a c-number field (i.e., quantized at tree level) and matter fields are fully quantized. The semiclassical theory may be summarized as follows.

Let $\left(\mathcal{M}, g_{a b}\right)$ be a globally hyperbolic four-dimensional spacetime manifold $\mathcal{M}$ with metric $g_{a b}$ and consider a real scalar quantum field $\phi$ of mass $m$ propagating on that manifold; we assume a scalar field for simplicity. The classical action $S_{m}$ for this matter field is given by the functional

$$
S_{m}[g, \phi]=-\frac{1}{2} \int d^{4} x \sqrt{-g}\left[g^{a b} \nabla_{a} \phi \nabla_{b} \phi+\left(m^{2}+\xi R\right) \phi^{2}\right],
$$

where $\nabla_{a}$ is the covariant derivative associated with the metric $g_{a b}, \xi$ is a coupling parameter between the field and the scalar curvature of the underlying spacetime $R$ and $g=\operatorname{det} g_{a b}$.

The field may be quantized in the manifold using the standard canonical quantization formalism [34, 121, 362]. The field operator in the Heisenberg representation $\hat{\phi}$ is an operator-valued distribution solution of the Klein-Gordon equation,

$$
\left(\square-m^{2}-\xi R\right) \hat{\phi}=0,
$$

where $\square=\nabla_{a} \nabla^{a}$. We may write the field operator as $\hat{\phi}[g ; x)$ to indicate that it is a functional of the metric $g_{a b}$ and a function of the spacetime point $x$. This notation will be used also for other operators and tensors.

The classical stress-energy tensor is obtained by functional derivation of this action in the usual way:

$$
T^{a b}(x)=\frac{2}{\sqrt{-g}} \frac{\delta S_{m}}{\delta g_{a b}},
$$

leading to

$$
\begin{aligned}
T^{a b}[g, \phi]= & \nabla^{a} \phi \nabla^{b} \phi-\frac{1}{2} g^{a b}\left(\nabla^{c} \phi \nabla_{c} \phi+m^{2} \phi^{2}\right) \\
& +\xi\left(g^{a b} \square-\nabla^{a} \nabla^{b}+G^{a b}\right) \phi^{2}
\end{aligned}
$$

where $G_{a b}$ is the Einstein tensor. With the notation $T^{a b}[g, \phi]$ we explicitly indicate that the stress-energy tensor is a functional of the metric $g_{a b}$ and the field $\phi$.

The next step is to define a stress-energy tensor operator $\hat{T}^{a b}[g ; x)$. Naively, one would replace the classical field $\phi[g ; x)$ in the above functional with the quantum operator $\hat{\phi}[g ; x)$, but this procedure involves taking the product of two distributions at the same spacetime point; this is illdefined and we need a regularization procedure. There are several regularization methods, which one may use. One is the point-splitting or point-separation regularization method [83, 84], in which one introduces a point $y$ in the neighborhood of the point $x$ and then uses as the regulator the 
vector tangent at point $x$ of the geodesic joining $x$ and $y$; this method is discussed in [303, 304, 305] and in Section 5. Another well-known method is dimensional regularization, in which one works in $n$ dimensions, where $n$ is not necessarily an integer, and then uses as the regulator the parameter $\epsilon=n-4$; this method is implicitly used in this section. The regularized stress-energy operator using the Weyl ordering prescription, i.e., symmetrical ordering, can be written as

$$
\hat{T}^{a b}[g]=\frac{1}{2}\left\{\nabla^{a} \hat{\phi}[g], \nabla^{b} \hat{\phi}[g]\right\}+\mathcal{D}^{a b}[g] \hat{\phi}^{2}[g],
$$

where $\mathcal{D}^{a b}[g]$ is the differential operator

$$
\mathcal{D}^{a b} \equiv(\xi-1 / 4) g^{a b} \square+\xi\left(R^{a b}-\nabla^{a} \nabla^{b}\right)
$$

Note that if dimensional regularization is used, the field operator $\hat{\phi}[g ; x)$ propagates in an $n$ dimensional spacetime. Once the regularization prescription has been introduced, a regularized and renormalized stress-energy operator $\hat{T}_{a b}^{R}[g ; x)$ may be defined as

$$
\hat{T}_{a b}^{R}[g ; x)=\hat{T}_{a b}[g ; x)+F_{a b}^{C}[g ; x) \hat{I},
$$

which differs from the regularized $\hat{T}_{a b}[g ; x)$ by the identity operator times some tensor counterterms $F_{a b}^{C}[g ; x)$, which depend on the regulator and are local functionals of the metric; see [258] for details. The field states can be chosen in such a way that for any pair of physically acceptable states, i.e., Hadamard states in the sense of [362], $|\psi\rangle$ and $|\varphi\rangle$, the matrix element $\left\langle\psi\left|T_{a b}^{R}\right| \varphi\right\rangle$, defined as the limit when the regulator takes the physical value, is finite and satisfies Wald's axioms [121, 359]. These counter-terms can be extracted from the singular part of a SchwingerDeWitt series [44, 83, 84, 121]. The choice of these counter-terms is not unique, but this ambiguity can be absorbed into the renormalized coupling constants, which appear in the equations of motion for the gravitational field.

The semiclassical Einstein equation for the metric $g_{a b}$ can then be written as

$$
G_{a b}[g]+\Lambda g_{a b}-2\left(\alpha A_{a b}+\beta B_{a b}\right)[g]=8 \pi G\left\langle\hat{T}_{a b}^{R}[g]\right\rangle
$$

where $\left\langle\hat{T}_{a b}^{R}[g]\right\rangle$ is the expectation value of the operator $\hat{T}_{a b}^{R}[g, x)$ after the regulator takes the physical value in some physically acceptable state of the field on $\left(\mathcal{M}, g_{a b}\right)$. Note that both the stress tensor and the quantum state are functionals of the metric, hence the notation. The parameters $G, \Lambda, \alpha$ and $\beta$ are the renormalized coupling constants, respectively the gravitational constant, the cosmological constant and two dimensionless coupling constants, which are zero in the classical Einstein equation. These constants must be understood as the result of "dressing" the bare constants, which appear in the classical action before renormalization. The values of these constants must be determined by experiment. The left-hand side of Equation (8) may be derived from the gravitational action

$$
S_{g}[g]=\frac{1}{8 \pi G} \int d^{4} x \sqrt{-g}\left[\frac{1}{2} R-\Lambda+\alpha C_{a b c d} C^{a b c d}+\beta R^{2}\right]
$$

where $C_{a b c d}$ is the Weyl tensor. The tensors $A_{a b}$ and $B_{a b}$ come from functional derivatives with respect to the metric of the terms quadratic in the curvature in Equation (9); they are explicitly 
given by

$$
\begin{aligned}
A^{a b}= & \frac{1}{\sqrt{-g}} \frac{\delta}{\delta g_{a b}} \int d^{4} \sqrt{-g} C_{c d e f} C^{c d e f} \\
= & \frac{1}{2} g^{a b} C_{c d e f} C^{c d e f}-2 R^{a c d e} R_{c d e}^{b}+4 R^{a c} R_{c}^{b}-\frac{2}{3} R R^{a b} \\
& -2 \square R^{a b}+\frac{2}{3} \nabla^{a} \nabla^{b} R+\frac{1}{3} g^{a b} \square R, \\
B^{a b}= & \frac{1}{\sqrt{-g}} \frac{\delta}{\delta g_{a b}} \int d^{4} \sqrt{-g} R^{2} \\
= & \frac{1}{2} g^{a b} R^{2}-2 R R^{a b}+2 \nabla^{a} \nabla^{b} R-2 g^{a b} \square R,
\end{aligned}
$$

where $R_{a b c d}$ and $R_{a b}$ are the Riemann and Ricci tensors, respectively. These two tensors are, like the Einstein and metric tensors, symmetric and divergence-less: $\nabla^{a} A_{a b}=0=\nabla^{a} B_{a b}$.

A solution of semiclassical gravity consists of a spacetime $\left(\mathcal{M}, g_{a b}\right)$, a quantum field operator $\hat{\phi}[g]$, which satisfies the evolution Equation (2) and a physically acceptable state $|\psi[g]\rangle$ for this field, such that Equation (8) is satisfied when the expectation value of the renormalized stress-energy operator is evaluated in this state.

For a free quantum field this theory is robust in the sense that it is self-consistent and fairly well understood. As long as the gravitational field is assumed to be described by a classical metric, the above semiclassical Einstein equations seem to be the only plausible dynamical equation for this metric: the metric couples to matter fields via the stress-energy tensor and for a given quantum state the only physically observable c-number stress-energy tensor that one can construct is the above renormalized expectation value. However, lacking a full quantum-gravity theory, the scope and limits of the theory are not so well understood. It is assumed that the semiclassical theory will break down at Planck scales, which is when simple order-of-magnitude estimates suggest that the quantum effects of gravity should not be ignored, because the energy of a quantum fluctuation in a Planck-size region, as determined by the Heisenberg uncertainty principle, is comparable to the gravitational energy of that fluctuation.

The theory is expected to break down when the fluctuations of the stress-energy operator are large [111]. A criterion based on the ratio of the fluctuations to the mean was proposed by Kuo and Ford [237] (see also work over zeta-function methods [86, 302]). This proposal was questioned by Phillips and $\mathrm{Hu}[198,303,304]$ because it does not contain a scale at which the theory is probed or how accurately the theory can be resolved. They suggested the use of a smearing scale or point-separation distance for integrating over the bitensor quantities, which is equivalent to a stipulation of the resolution level of measurements; see also the response by Ford [113, 115]. A different criterion was recently suggested by Anderson et al. [10, 11] based on linear-response theory. A partial summary of this issue can be found in our Erice Lectures [207].

More recently, in collaboration with A. Roura [203, 204], we have proposed a criterion for the validity of semiclassical gravity, which is based on the stability of the solutions of the semiclassical Einstein equations with respect to quantum metric fluctuations. The two-point correlations for the metric perturbations can be described in the framework of stochastic gravity, which is closely related to the quantum theory of gravity interacting with $N$ matter fields, to leading order in a $1 / N$ expansion. We will describe these developments in the following sections.

\subsection{Stochastic gravity}

The purpose of stochastic gravity is to extend semiclassical theory to account for these fluctuations in a self-consistent way. A physical observable that describes these fluctuations to lowest order is 
the noise kernel bitensor, which is defined through the two-point correlation of the stress-energy operator as

$$
N_{a b c d}[g ; x, y)=\frac{1}{2}\left\langle\left\{\hat{t}_{a b}[g ; x), \hat{t}_{c d}[g ; y)\right\}\right\rangle,
$$

where the curly brackets mean anticommutator, and where

$$
\hat{t}_{a b}[g ; x) \equiv \hat{T}_{a b}[g ; x)-\left\langle\hat{T}_{a b}[g ; x)\right\rangle .
$$

This bitensor can also be written $N_{a b, c^{\prime} d^{\prime}}[g ; x, y)$, or $N_{a b, c^{\prime} d^{\prime}}(x, y)$ as we do in Section 5 , to emphasize that it is a tensor with respect to the first two indices at the point $x$ and a tensor with respect to the last two indices at the point $y$, but we shall not follow this notation here. The noise kernel is defined in terms of the unrenormalized stress-tensor operator $\hat{T}_{a b}[g ; x)$ on a given background metric $g_{a b}$, thus a regulator is implicitly assumed on the right-hand side of Equation (12). However, for a linear quantum field, the above kernel - the expectation function of a bitensor - is free of ultraviolet divergences because the regularized $T_{a b}[g ; x)$ differs from the renormalized $T_{a b}^{R}[g ; x)$ by the identity operator times some tensor counterterms (see Equation (7)) so that in the subtraction (13) the counterterms cancel. Consequently the ultraviolet behavior of $\left\langle\hat{T}_{a b}(x) \hat{T}_{c d}(y)\right\rangle$ is the same as that of $\left\langle\hat{T}_{a b}(x)\right\rangle\left\langle\hat{T}_{c d}(y)\right\rangle$, and $\hat{T}_{a b}$ can be replaced by the renormalized operator $\hat{T}_{a b}^{R}$ in Equation (12); an alternative proof of this result is given in [304, 305]. The noise kernel should be thought of as a distribution function, the limit of coincidence points has meaning only in the sense of distributions. The bitensor $N_{a b c d}[g ; x, y)$, or $N_{a b c d}(x, y)$ for short, is real and positive semi-definite, as a consequence of $\hat{T}_{a b}^{R}$ being self-adjoint. A simple proof is given in [208].

Once the fluctuations of the stress-energy operator have been characterized, we can perturbatively extend the semiclassical theory to account for such fluctuations. Thus we will assume that the background spacetime metric $g_{a b}$ is a solution of the semiclassical Einstein equations (8) and we will write the new metric for the extended theory as $g_{a b}+h_{a b}$, where we will assume that $h_{a b}$ is a perturbation to the background solution. The renormalized stress-energy operator and the state of the quantum field may now be denoted by $\hat{T}_{a b}^{R}[g+h]$ and $|\psi[g+h]\rangle$, respectively, and $\left\langle\hat{T}_{a b}^{R}[g+h]\right\rangle$ will be the corresponding expectation value.

Let us now introduce a Gaussian stochastic tensor field $\xi_{a b}[g ; x)$ defined by the following correlators:

$$
\left\langle\xi_{a b}[g ; x)\right\rangle_{s}=0, \quad\left\langle\xi_{a b}[g ; x) \xi_{c d}[g ; y)\right\rangle_{s}=N_{a b c d}[g ; x, y),
$$

where $\langle\ldots\rangle_{s}$ means statistical average. The symmetry and positive semi-definite property of the noise kernel guarantees that the stochastic field tensor $\xi_{a b}[g, x)$, or $\xi_{a b}(x)$ for short, just introduced is well-defined. Note that this stochastic tensor captures only partially the quantum nature of the fluctuations of the stress-energy operator as it assumes that cumulants of higher order are zero.

An important property of this stochastic tensor is that it is covariantly conserved in the background spacetime $\nabla^{a} \xi_{a b}[g ; x)=0$. In fact, as a consequence of the conservation of $\hat{T}_{a b}^{R}[g]$ one can see that $\nabla_{x}^{a} N_{a b c d}(x, y)=0$. Taking the divergence in Equation (14) one can then show that $\left\langle\nabla^{a} \xi_{a b}\right\rangle_{s}=0$ and $\left\langle\nabla_{x}^{a} \xi_{a b}(x) \xi_{c d}(y)\right\rangle_{s}=0$ so that $\nabla^{a} \xi_{a b}$ is deterministic and represents with certainty the zero vector field in $\mathcal{M}$.

For a conformal field, i.e., a field whose classical action is conformally invariant, $\xi_{a b}$ is traceless: $g^{a b} \xi_{a b}[g ; x)=0$; so that, for a conformal matter field the stochastic source gives no correction to the trace anomaly. In fact, from the trace anomaly result, which states that $g^{a b} \hat{T}_{a b}^{R}[g]$ is, in this case, a local c-number functional of $g_{a b}$ times the identity operator, we have that $g^{a b}(x) N_{a b c d}[g ; x, y)=0$. It then follows from Equation (14) that $\left\langle g^{a b} \xi_{a b}\right\rangle_{s}=0$ and $\left\langle g^{a b}(x) \xi_{a b}(x) \xi_{c d}(y)\right\rangle_{s}=0$; an alternative proof based on the point-separation method is given in [304, 305], see also Section 5.

All these properties make it quite natural to incorporate into the Einstein equations the stressenergy fluctuations by using the stochastic tensor $\xi_{a b}[g ; x)$ as the source of the metric perturbations. 
Thus we will write the following equation:

$$
G_{a b}[g+h]+\Lambda\left(g_{a b}+h_{a b}\right)-2\left(\alpha A_{a b}+\beta B_{a b}\right)[g+h]=8 \pi G\left(\left\langle\hat{T}_{a b}^{R}[g+h]\right\rangle+\xi_{a b}[g]\right) .
$$

This equation is in the form of a (semiclassical) Einstein-Langevin equation; it is a dynamical equation for the metric perturbation $h_{a b}$ to linear order. It describes the backreaction of the metric to the quantum fluctuations of the stress-energy tensor of matter fields, and gives a firstorder extension to semiclassical gravity as described by the semiclassical Einstein equation (8).

Note that we refer to the Einstein-Langevin equation as a first-order extension to the semiclassical Einstein equation of semiclassical gravity and the lowest-level representation of stochastic gravity. However, stochastic gravity has a much broader meaning; it refers to the range of theories based on second and higher-order correlation functions. Noise can be defined in effectively-open systems (e.g., correlation noise [61] in the Schwinger-Dyson equation hierarchy) to some degree, but one should not expect the Langevin form to prevail. In this sense we say stochastic gravity is the intermediate theory between semiclassical gravity (a mean field theory based on the expectation values of the energy momentum tensor of quantum fields) and quantum gravity (the full hierarchy of correlation functions retaining complete quantum coherence [187, 188].

The renormalization of the operator $\hat{T}_{a b}[g+h]$ is carried out exactly as in the previous case, now in the perturbed metric $g_{a b}+h_{a b}$. Note that the stochastic source $\xi_{a b}[g ; x)$ is not dynamical; it is independent of $h_{a b}$, since it describes the fluctuations of the stress tensor on the semiclassical background $g_{a b}$.

An important property of the Einstein-Langevin equation is that it is gauge invariant under the change of $h_{a b}$ by $h_{a b}^{\prime}=h_{a b}+\nabla_{a} \zeta_{b}+\nabla_{b} \zeta_{a}$, where $\zeta^{a}$ is a stochastic vector field on the background manifold $\mathcal{M}$. Note that a tensor such as $R_{a b}[g+h]$ transforms as $R_{a b}\left[g+h^{\prime}\right]=R_{a b}[g+h]+\mathcal{L}_{\zeta} R_{a b}[g]$ to linear order in the perturbations, where $\mathcal{L}_{\zeta}$ is the Lie derivative with respect to $\zeta^{a}$. Now, let us write the source tensors in Equations (15) and (8) to the left-hand sides of these equations. If we substitute $h$ with $h^{\prime}$ in this new version of Equation (15), we get the same expression, with $h$ instead of $h^{\prime}$, plus the Lie derivative of the combination of tensors, which appear on the left-hand side of the new Equation (8). This last combination vanishes when Equation (8) is satisfied, i.e., when the background metric $g_{a b}$ is a solution of semiclassical gravity.

From the statistical average of Equation (15) we have that $g_{a b}+\left\langle h_{a b}\right\rangle_{s}$ must be a solution of the semiclassical Einstein equation linearized around the background $g_{a b}$; this solution has been proposed as a test for the validity of the semiclassical approximation $[10,11]$ a point that will be further discussed in Section 3.3.

The stochastic equation (15) predicts that the gravitational field has stochastic fluctuations over the background $g_{a b}$. This equation is linear in $h_{a b}$, thus its solutions can be written as follows,

$$
h_{a b}(x)=h_{a b}^{0}(x)+8 \pi G \int d^{4} x^{\prime} \sqrt{-g\left(x^{\prime}\right)} G_{a b c d}^{\mathrm{ret}}\left(x, x^{\prime}\right) \xi^{c d}\left(x^{\prime}\right),
$$

where $h_{a b}^{0}(x)$ is the solution of the homogeneous equation containing information on the initial conditions and $G_{a b c d}^{\mathrm{ret}}\left(x, x^{\prime}\right)$ is the retarded propagator of Equation (15) with vanishing initial conditions. Form this we obtain the two-point correlation functions for the metric perturbations:

$$
\begin{aligned}
\left\langle h_{a b}(x) h_{c d}(y)\right\rangle_{s}= & \left\langle h_{a b}^{0}(x) h_{c d}^{0}(y)\right\rangle_{s}+ \\
& (8 \pi G)^{2} \int d^{4} x^{\prime} d^{4} y^{\prime} \sqrt{g\left(x^{\prime}\right) g\left(y^{\prime}\right)} G_{a b e f}^{\mathrm{ret}}\left(x, x^{\prime}\right) N^{e f g h}\left(x^{\prime}, y^{\prime}\right) G_{c d g h}^{\mathrm{ret}}\left(y, y^{\prime}\right) \\
\equiv & \left\langle h_{a b}(x) h_{c d}(y)\right\rangle_{\mathrm{int}}+\left\langle h_{a b}(x) h_{c d}(y)\right\rangle_{\mathrm{ind}} .
\end{aligned}
$$

There are two different contributions to the two-point correlations, which we have distinguished in the second equality. The first one is connected to the fluctuations of the initial state of the 
metric perturbations and we will refer to them as intrinsic fluctuations. The second contribution is proportional to the noise kernel and is thus connected with the fluctuations of the quantum fields; we will refer to them as induced fluctuations. To find these two-point stochastic correlation functions one needs to know the noise kernel $N_{a b c d}(x, y)$. Explicit expressions of this kernel in terms of the two-point Wightman functions is given in [258], expressions based on point-splitting methods have also been given in [304, 315]. Note that the noise kernel should be thought of as a distribution function, the limit of coincidence points has meaning only in the sense of distributions.

The two-point stochastic correlation functions for the metric perturbations of Equation (17) satisfy a very important property. In fact, it can be shown that they correspond exactly to the symmetrized two-point correlation functions for the quantum metric perturbations in the large $N$ expansion, i.e., the quantum theory describing the interaction of the gravitational field with $N$ arbitrary free fields and expanded in powers of $1 / N$. To leading order for the graviton propagator one finds that

$$
\left\langle\left\{\hat{h}_{a b}(x), \hat{h}_{c d}(y)\right\}\right\rangle=2\left\langle h_{a b}(x) h_{c d}(y)\right\rangle_{s},
$$

where $\hat{h}_{a b}(x)$ is the quantum operator corresponding to the metric perturbations and the statistical average in Equation (17) for the homogeneous solutions is now taken with respect to the Wigner distribution that describes the initial quantum state of the metric perturbations. The Lorentz gauge condition $\nabla^{a}\left(h_{a b}-(1 / 2) \eta_{a b} h_{c}^{c}\right)=0$, as well as an initial condition to completely fix the gauge of the initial state, should be implicitly understood. Moreover, since there are now $N$ scalar fields, the stochastic source has been rescaled so that the two-point correlation defined by Equation (14) should be $1 / N$ times the noise kernel of a single field. This result was implicitly obtained in the Minkowski background in [259] where the two-point correlation in the stochastic context was computed for the linearized metric perturbations. This stochastic correlation exactly agrees with the symmetrized part of the graviton propagator computed by Tomboulis [348] in the quantum context of gravity interacting with $N$ Fermion fields, where the graviton propagator is of order $1 / N$. This result can be extended to an arbitrary background in the context of the large $N$ expansion; a sketch of the proof with explicit details in the Minkowski background can be found in [203]. This connection between the stochastic correlations and the quantum correlations was noted and studied in detail in the context of simpler open quantum systems [66]. Stochastic gravity goes beyond semiclassical gravity in the following sense. The semiclassical theory, which is based on the expectation value of the stress-energy tensor, carries information on the field two-point correlations only, since $\left\langle\hat{T}_{a b}\right\rangle$ is quadratic in the field operator $\hat{\phi}$. The stochastic theory, on the other hand, is based on the noise kernel 12, which is quartic in the field operator. However, it does not carry information on the graviton-graviton interaction, which in the context of the large $N$ expansion gives diagrams of order $1 / N^{2}$. This will be illustrated in Section 3.3.1. Furthermore, the retarded propagator also gives information on the commutator

$$
\left\langle\left[\hat{h}_{a b}(x), \hat{h}_{c d}(y)\right]\right\rangle=16 \pi i G\left(G_{a b c d}^{\mathrm{ret}}(y, x)-G_{a b c d}^{\mathrm{ret}}(x, y)\right),
$$

so that combining the commutator with the anticommutator, the quantum two-point correlation functions are determined. Moreover, assuming a Gaussian initial state with vanishing expectation value for the metric perturbations, any $n$-point quantum correlation function is determined by the two-point quantum correlations and thus by the stochastic approach. Consequently, one may regard the Einstein-Langevin equation as a useful intermediary tool to compute the correlation functions for the quantum metric perturbations.

We should, however, also emphasize that Langevin-like equations are obtained to describe the quantum to classical transition in open quantum systems, when quantum decoherence takes place by coarse-graining of the environment as well as by suitable coarse-graining of the system variables [101, 127, 144, 146, 150, 374]. In those cases the stochastic correlation functions describe actual classical correlations of the system variables. Examples can be found in the case of a 
moving charged particle in an electromagnetic field in quantum electrodynamics [219] and in several quantum Brownian models [64, 65, 66].

\subsection{Validity of semiclassical gravity}

As we have emphasized earlier, the scope and limits of semiclassical gravity are not well understood because we still lack a fully well-understood quantum theory of gravity. From the semiclassical Einstein equations it also seems clear that the semiclassical theory should break down when the quantum fluctuations of the stress tensor are large. Ford [111] was among the first to have emphasized the importance of these quantum fluctuations. It is less clear, however, how to quantify the size of these fluctuations. Kuo and Ford [237] used the variance of the fluctuations of the stress tensor operator compared to the mean value as a measure of the validity of semiclassical gravity. $\mathrm{Hu}$ and Phillips pointed out $[198,303]$ that such a criterion should be refined by considering the backreaction of those fluctuations on the metric. Ford and collaborators also noticed that the metric fluctuations associated with the matter fluctuations can be meaningfully classified as $a c$ tive [114, 384, 385] and passive [111, 112, 115, 237], which correspond to our intrinsic and induced fluctuations, respectively, and have studied their properties in different contexts [35, 36, 37]. However, these fluctuations are not treated in a unified way and their precise relation to the quantum correlation function for the metric perturbations is not discussed. Furthermore, the full-averaged backreaction of the matter fields is not included self-consistently and the contribution from the vacuum fluctuations in Minkowski space is discarded.

A different approach to the validity of semiclassical gravity was pioneered by Horowitz [169, 170], who studied the stability of a semiclassical solution with respect to linear metric perturbations. In the case of a free quantum matter field in its Minkowski vacuum state, flat spacetime is a solution of semiclassical gravity. The equations describing those metric perturbations involve higher-order derivatives and Horowitz found unstable runaway solutions that grow exponentially with characteristic timescales comparable to the Planck time; see also the analysis by Jordan [223]. Later, Simon [329, 330] argued that those unstable solutions lie beyond the expected domain of validity of the theory and emphasized that only those solutions, which resulted from truncating perturbative expansions in terms of the square of the Planck length, are physically acceptable [329, 330]. Further discussion was provided by Flanagan and Wald [110], who advocated the use of an order-reduction prescription first introduced by Parker and Simon [295]. More recently Anderson, Molina-París and Mottola have taken up the issue of the validity of semiclassical gravity [10, 11] again. Their starting point is the fact that the semiclassical Einstein equation will fail to provide a valid description of the dynamics of the mean spacetime geometry whenever the higherorder radiative corrections to the effective action, involving loops of gravitons or internal graviton propagators, become important. Next, they argue qualitatively that such higher-order radiative corrections cannot be neglected if the metric fluctuations grow without bound. Finally, they propose a criterion to characterize the growth of the metric fluctuations, and hence the validity of semiclassical gravity, based on the stability of the solutions of the linearized semiclassical equation. Following these approaches, the Minkowski metric is shown to be a stable solution of semiclassical gravity with respect to small metric perturbations.

As emphasized in $[10,11]$ the above criteria may be understood as based on semiclassical gravity itself. It is certainly true that stability is a necessary condition for the validity of a semiclassical solution, but one may also look for criteria within extensions of semiclassical gravity. In the absence of a quantum theory of gravity, such criteria may be found in some more modest extensions. Thus, Ford [111] considered graviton production in linearized quantum gravity and compared the results with the production of gravitational waves in semiclassical gravity. Ashtekar [13] and Beetle [22] found large quantum-gravity effects in three-dimensional quantum-gravity models. In a more recent paper [203] (see also [204]), we advocate for a criteria within the stochastic gravity approach and 
since stochastic gravity extends semiclassical gravity by incorporating the quantum stress-tensor fluctuations of the matter fields, this criteria is structurally the most complete to date.

It turns out that this validity criteria is equivalent to the validity criteria that one might advocate within the large $N$ expansion; that is the quantum theory describing the interaction of the gravitational field with $N$ identical free matter fields. In the leading order, namely the limit in which $N$ goes to infinity and the gravitational constant is appropriately rescaled, the theory reproduces semiclassical gravity. Thus, a natural extension of semiclassical gravity is provided by the next to leading order. It turns out that the symmetrized two-point quantum-correlation functions of the metric perturbations in the large $N$ expansion are equivalent to the two-point stochastic metric-correlation functions predicted by stochastic gravity. Our validity criterion can then be formulated as follows: a solution of semiclassical gravity is valid when it is stable with respect to quantum metric perturbations. This criterion involves the consideration of quantumcorrelation functions of the metric perturbations, since the quantum field describing the metric perturbations $\hat{h}_{a b}(x)$ is characterized not only by its expectation value but also by its $n$-point correlation functions.

It is important to emphasize that the above validity criterion incorporates in a unified and selfconsistent way the two main ingredients of the criteria exposed above. Namely, the criteria based on the quantum stress-tensor fluctuations of the matter fields, and the criteria based on the stability of semiclassical solutions against classical metric perturbations. The former is incorporated through the induced metric fluctuations, and the later through the intrinsic fluctuations introduced in Equation (17). Whereas information on the stability of intrinsic-metric fluctuations can be obtained from an analysis of the solutions of the perturbed semiclassical Einstein equation (the homogeneous part of Equation (15)), the effect of induced-metric fluctuations is accounted for only in stochastic gravity (the full inhomogeneous Equation (15)). We will illustrate these criteria in Section 6.5 by studying the stability of Minkowski spacetime as a solution of semiclassical gravity.

\subsubsection{The large $\mathrm{N}$ expansion}

To illustrate the relation between the semiclassical, stochastic and quantum theories, a simplified model of scalar gravity interacting with $N$ scalar fields is considered here.

The large $N$ expansion has been successfully used in quantum chromodynamics to compute some nonperturbative results. This expansion re-sums and rearranges Feynman perturbative series, including self-energies. For gravity interacting with $N$ matter fields, it shows that graviton loops are of higher order than matter loops. To illustrate the large $N$ expansion, let us first consider the following toy model of gravity, which we will simplify as a scalar field $h$, interacting with a single scalar field $\phi$ described by the action

$$
\begin{aligned}
S= & \frac{1}{\kappa} \int d^{4} x\left(\partial_{a} h \partial^{a} h+h \partial_{a} h \partial^{a} h+\ldots\right) \\
& -\int d^{4} x\left(\partial_{a} \phi \partial^{a} \phi+m^{2} \phi^{2}\right)+\int d^{4} x\left(h \partial_{a} \phi \partial^{a} \phi+\ldots\right),
\end{aligned}
$$

where $\kappa=8 \pi G$ and we have assumed that the interaction is linear in the (dimensionless) scalar gravitational field $h$ and quadratic in the matter field $\phi$ to simulate in a simplified way the coupling of the metric with the stress tensor of the matter fields. We have also included a self-coupling graviton term of $O\left(h^{3}\right)$, which also appears in perturbative gravity beyond the linear approximation.

We may now compute the graviton dressed propagator perturbatively as the following series of Feynman diagrams (Figure 1). The first diagram is just the free graviton propagator, which is of $O(\kappa)$, as one can see from the kinetic term for the graviton in Equation (20). The next diagram is one loop of matter with two external legs, which are the graviton propagators. This diagram has two vertices with one graviton propagator and two matter field propagators. Since the vertices 
and the matter propagators contribute with 1 and each graviton propagator contributes with a $\kappa$ this diagram is of order $O\left(\kappa^{2}\right)$. The next diagram contains two loops of matter, three gravitons and consequently it is of order $O\left(\kappa^{3}\right)$. There will also be terms with one graviton loop and two graviton propagators as external legs and three graviton propagators at the two vertices due to the $O\left(h^{3}\right)$ term in the action (20). Since there are four graviton propagators, which carry a $\kappa^{4}$, but two vertices, which have $\kappa^{-2}$, this diagram is of order $O\left(\kappa^{2}\right)$, like the term with one matter loop. Thus, in this perturbative expansion, a graviton loop and a matter loop both contribute at the same order to the dressed graviton propagator.

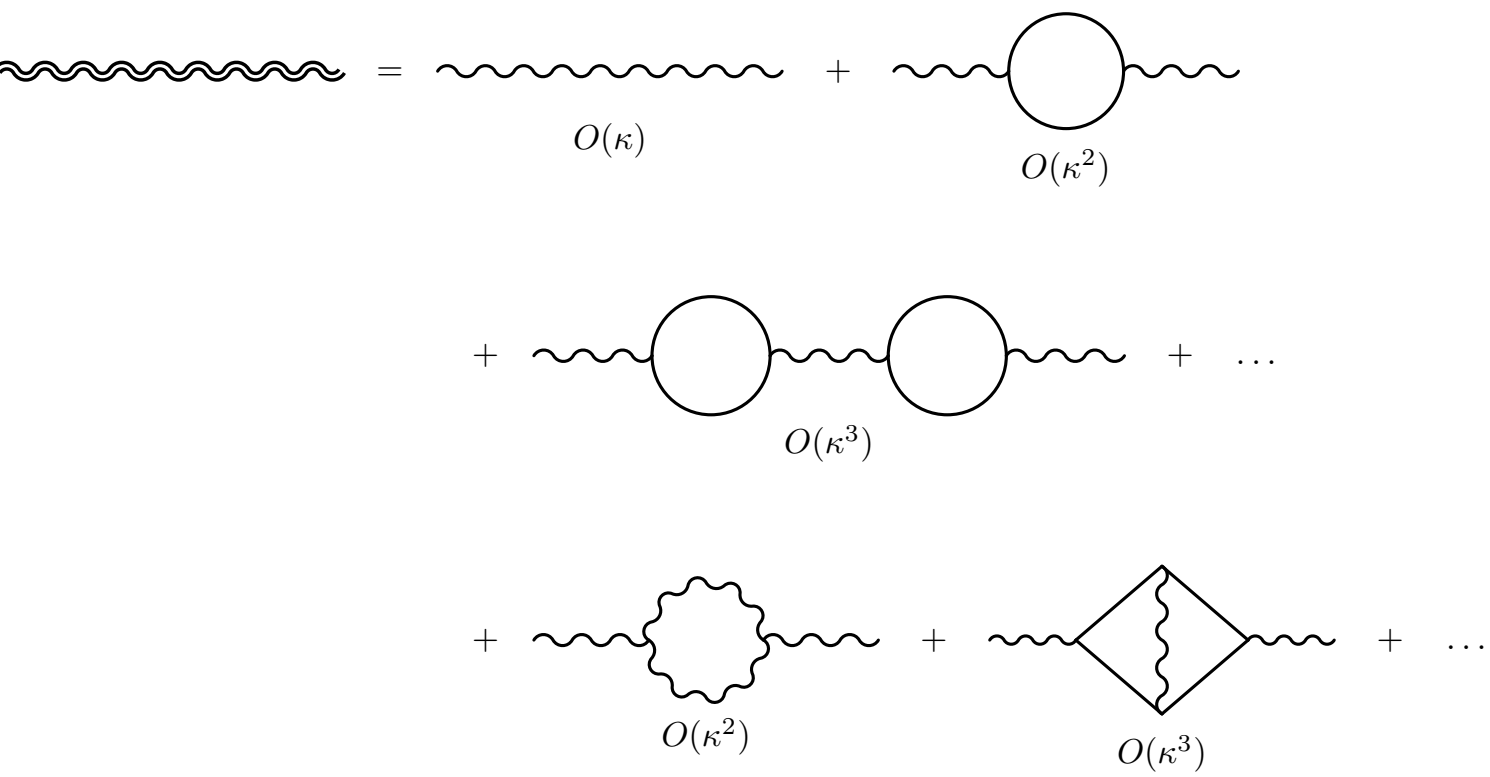

Figure 1: Series of Feynman diagrams expanded in powers of $\kappa$ for the dressed propagator of the graviton interacting with a single matter field according to the toy model described by the action (20).

Let us now consider the large $N$ expansion. We assume that the gravitational field is coupled with a large number of identical fields $\phi_{j}, j=1, \ldots, N$, which couple only with $h$. Next we rescale the gravitational coupling in such a way that $\bar{\kappa}=\kappa N$ is finite even when $N$ goes to infinity. The action of this system is:

$$
\begin{aligned}
S= & \frac{N}{\bar{\kappa}} \int d^{4} x\left(\partial_{a} h \partial^{a} h+h \partial_{a} h \partial^{a} h+\ldots\right) \\
& -\sum_{j}^{N} \int d^{4} x\left(\partial_{a} \phi_{j} \partial^{a} \phi_{j}+m^{2} \phi^{2}\right)+\sum_{j}^{N} \int d^{4} x\left(h \partial_{a} \phi_{j} \partial^{a} \phi_{j}+\ldots\right) .
\end{aligned}
$$

Now an expansion in powers of $1 / N$ of the dressed graviton propagator is given by the following series of Feynman diagrams (Figure 2). The first diagram is the free graviton propagator, which is now of order $O(\bar{\kappa} / N)$. The following diagrams are $N$ identical Feynman diagrams with one loop of matter and two graviton propagators as external legs, each diagram, due to the two graviton propagators, is of order $O\left(\bar{\kappa}^{2} / N^{2}\right)$, but since there are $N$ of them the sum can be represented by a single diagram with a loop of matter of weight $N$, and therefore this diagram is of order $O\left(\bar{\kappa}^{2} / N\right)$. This means that it is of the same order as the first diagram in an expansion in $1 / N$. Then there are the diagrams with two loops of matter and three graviton propagators, as before we can assign 
a weight of $N$ to each loop and taking into account the three graviton propagators this diagram is of order $O\left(\bar{\kappa}^{3} / N\right)$, and so on. This means that to order $1 / N$ the dressed graviton propagator contains all the perturbative series in powers of $\bar{\kappa}$ of the matter loops.

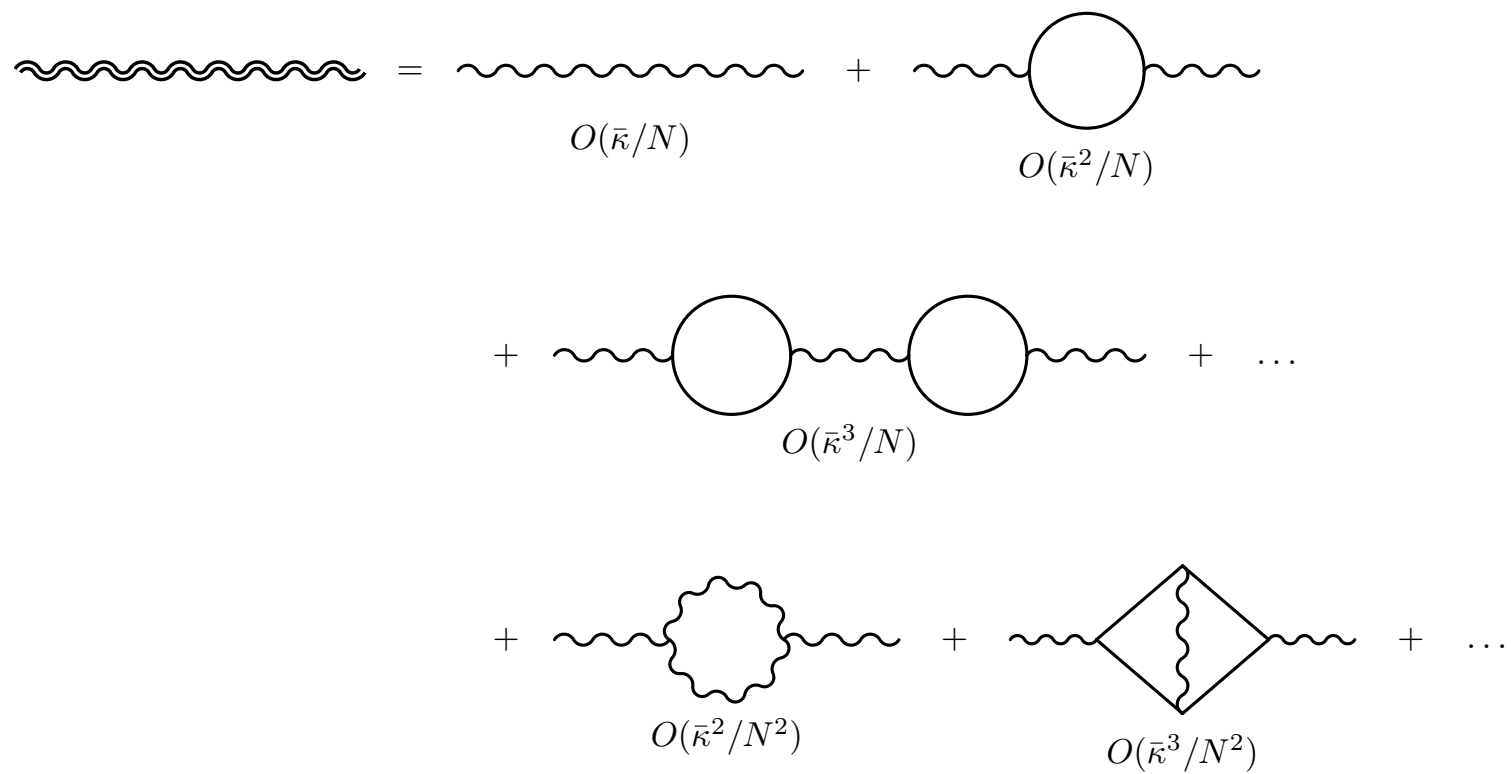

Figure 2: Series of Feynman diagrams expanded in powers of $1 / N$ for the dressed propagator of the graviton interacting with $N$ matter fields oaccording to the toy model described by the action (21).

Next, there is a diagram with one graviton loop and two graviton legs. Let us count the order of this diagram: it contains four graviton propagators and two vertices, the propagators contribute as $(\bar{\kappa} / N)^{4}$ and the vertices as $(N / \bar{\kappa})^{2}$, thus this diagram is of $O\left(\bar{\kappa}^{2} / N^{2}\right)$. Therefore graviton loops contribute to a higher order in the $1 / N$ expansion than matter loops. Similarly there are $N$ diagrams with one loop of matter with an internal graviton propagator and two external graviton legs. Thus we have three graviton propagators and, since there are $N$ of them, their sum is of order $O\left(\bar{\kappa}^{3} / N^{2}\right)$. To summarize, we have that when $N \rightarrow \infty$ there are no graviton propagators and gravity is classical, yet the matter fields are quantized. This is semiclassical gravity as was first described in [152]. Then we go to the next-to-leading order $1 / N$. Now the graviton propagator includes all matter-loop contributions, but no contributions from graviton loops or internal graviton propagators in matter loops. This is what stochastic gravity reproduces.

That stochastic gravity is connected to the large $N$ expansion can be seen from the stochastic correlations of linear metric perturbations on the Minkowski background computed in [259]. These correlations are in exact agreement with the imaginary part of the graviton propagator found by Tomboulis in the large $N$ expansion for the quantum theory of gravity interacting with $N$ Fermio fields [348]. This has been proven in detail in [203]; see also [204], where the case of a general background is also briefly discussed. 


\section{The Einstein-Langevin Equation: Functional Approach}

The Einstein-Langevin equation (15) may also be derived by a method based on functional techniques [258]. Here we will summarize these techniques starting with semiclassical gravity.

In semiclassical gravity functional methods were used to study the backreaction of quantum fields in cosmological models $[109,147,153]$. The primary advantage of the effective-action approach is, in addition to the well-known fact that it is easy to introduce perturbation schemes like loop expansion and nPI formalisms, that it yields a fully self-consistent solution. (For a general discussion in the semiclassical context of these two approaches, equation of motion versus effective action, contrast, e.g., [137, 193, 194, 251] with the above references and [3, 4, 148, 154]. See also comments in Section 8 on the black-hole backreaction problem comparing the approach by York et al. [380, 381, 382] to that of Sinha, Raval and Hu [332].

The well known in-out effective-action method treated in textbooks, however, led to equations of motion, which were not real because they were tailored to compute transition elements of quantum operators rather than expectation values. The correct technique to use for the backreaction problem is the Schwinger-Keldysh [14, 56, 82, 87, 227, 323, 343] closed-time-path (CTP) or 'in-in' effective action. These techniques were adapted to the gravitational context $[54,72,94,222,223,296]$ and applied to different problems in cosmology. One could deduce the semiclassical Einstein equation from the CTP effective action for the gravitational field (at tree level) with quantum matter fields.

Furthermore, in this case the CTP functional formalism turns out to be related [58, 69, 70, $73,134,239,247,256,258,267,343]$ to the influence-functional formalism of Feynman and Vernon [108], since the full quantum system may be understood as consisting of a distinguished subsystem (the "system" of interest) interacting with the remaining degrees of freedom (the environment). Integrating out the environment variables in a CTP path integral yields the influence functional, from which one can define an effective action for the dynamics of the system [58, 134, 191, 206]. This approach to semiclassical gravity is motivated by the observation [181] that in some open quantum systems classicalization and decoherence [46, 131, 221, 352, 389, 390, 391, 392, 393] on the system may be brought about by interaction with an environment, the environment being in this case the matter fields and some "high-momentum" gravitational modes [50, 51, 143, 182, 195, 231, 283, 374]. Unfortunately, since the form of a complete quantum theory of gravity interacting with matter is unknown, we do not know what these "high-momentum" gravitational modes are. Such a fundamental quantum theory might not even be a field theory, in which case the metric and scalar fields would not be fundamental objects [187]. Thus, in this case, we cannot attempt to evaluate the influence action of Feynman and Vernon starting from the fundamental quantum theory and performing the path integrations in the environment variables. Instead, we introduce the influence action for an effective quantum field theory of gravity and matter [95, 96, 97, 98, 297, 298, 331], in which such "high-momentum" gravitational modes are assumed to have already been "integrated out."

\subsection{Influence action for semiclassical gravity}

Let us formulate semiclassical gravity in this functional framework. Adopting the usual procedure of effective field theories [63,95, 96, 97, 98, 366, 367], one has to take the effective action for the metric and the scalar field of the most general local form compatible with general covariance: $S[g, \phi] \equiv S_{\mathrm{g}}[g]+S_{\mathrm{m}}[g, \phi]+\ldots$, where $S_{\mathrm{g}}[g]$ and $S_{\mathrm{m}}[g, \phi]$ are given by Equations (9) and (1), respectively, and the dots stand for terms of order higher than two in the curvature and in the number of derivatives of the scalar field. Here, we shall neglect the higher-order terms as well as self-interaction terms for the scalar field. The second order terms are necessary to renormalize one-loop ultraviolet divergences of the scalar field stress-energy tensor, as we have already seen. Since $\mathcal{M}$ is a globally hyperbolic manifold, we can foliate it by a family of $t=$ constant Cauchy 
hypersurfaces $\Sigma_{t}$, and we will indicate the initial and final times by $t_{\mathrm{i}}$ and $t_{\mathrm{f}}$, respectively.

The influence functional corresponding to the action (1) describing a scalar field in a spacetime (coupled to a metric field) may be introduced as a functional of two copies of the metric, denoted by $g_{a b}^{+}$and $g_{a b}^{-}$, which coincide at some final time $t=t_{\mathrm{f}}$. Let us assume that, in the quantum effective theory, the state of the full system (the scalar and the metric fields) in the Schrödinger picture at the initial time $t=t_{\mathrm{i}}$ can be described by a density operator, which can be written as the tensor product of two operators on the Hilbert spaces of the metric and of the scalar field. Let $\rho_{\mathrm{i}}\left(t_{\mathrm{i}}\right) \equiv \rho_{\mathrm{i}}\left[\phi_{+}\left(t_{\mathrm{i}}\right), \phi_{-}\left(t_{\mathrm{i}}\right)\right]$ be the matrix element of the density operator $\hat{\rho}^{\mathrm{S}}\left(t_{\mathrm{i}}\right)$ describing the initial state of the scalar field. The Feynman-Vernon influence functional is defined as the following path integral over the two copies of the scalar field:

$$
\mathcal{F}_{\mathrm{IF}}\left[g^{ \pm}\right] \equiv \int \mathcal{D} \phi_{+} \mathcal{D} \phi_{-} \rho_{\mathrm{i}}\left(t_{\mathrm{i}}\right) \delta\left[\phi_{+}\left(t_{\mathrm{f}}\right)-\phi_{-}\left(t_{\mathrm{f}}\right)\right] e^{i\left(S_{\mathrm{m}}\left[g^{+}, \phi_{+}\right]-S_{\mathrm{m}}\left[g^{-}, \phi_{-}\right]\right)}
$$

Alternatively, the above double path integral can be rewritten as a CTP integral, namely, as a single path integral in a complex time contour with two different time branches, one going forward in time from $t_{\mathrm{i}}$ to $t_{\mathrm{f}}$ and the other going backward in time from $t_{\mathrm{f}}$ to $t_{\mathrm{i}}$ (in practice one usually takes $\left.t_{\mathrm{i}} \rightarrow-\infty\right)$. From this influence functional, the influence action $S_{\mathrm{IF}}\left[g^{+}, g^{-}\right]$, or $S_{\mathrm{IF}}\left[g^{ \pm}\right]$for short, defined by

$$
\mathcal{F}_{\mathrm{IF}}\left[g^{ \pm}\right] \equiv e^{i S_{\mathrm{IF}}\left[g^{ \pm}\right]}
$$

carries all the information about the environment (the matter fields) relevant to the system (the gravitational field). Then we can define the CTP effective action for the gravitational field, $S_{\text {eff }}\left[g^{ \pm}\right]$, as

$$
S_{\text {eff }}\left[g^{ \pm}\right] \equiv S_{\mathrm{g}}\left[g^{+}\right]-S_{\mathrm{g}}\left[g^{-}\right]+S_{\mathrm{IF}}\left[g^{ \pm}\right]
$$

This is the effective action for the classical gravitational field in the CTP formalism. However, since the gravitational field is treated only at the tree level, this is also the effective classical action from which the classical equations of motion can be derived.

Expression (22) contains ultraviolet divergences and must be regularized. We shall assume that dimensional regularization can be applied, that is, it makes sense to dimensionally continue all the quantities that appear in Equation (22). For this we need to work with the $n$-dimensional actions corresponding to $S_{\mathrm{m}}$ in Equation (22) and $S_{\mathrm{g}}$ in Equation (9). For example, the parameters $G$, $\Lambda, \alpha$, and $\beta$ of Equation (9) are the bare parameters $G_{\mathrm{B}}, \Lambda_{\mathrm{B}}, \alpha_{\mathrm{B}}$, and $\beta_{\mathrm{B}}$, and in $S_{\mathrm{g}}[g]$, instead of the square of the Weyl tensor in Equation (9), one must use $\frac{2}{3}\left(R_{a b c d} R^{a b c d}-R_{a b} R^{a b}\right)$, which by the Gauss-Bonnet theorem leads to the same equations of motion as the action (9) when $n=4$. The form of $S_{\mathrm{g}}$ in $n$ dimensions is suggested by the Schwinger-DeWitt analysis of the ultraviolet divergences in the matter stress-energy tensor using dimensional regularization. One can then write the Feynman-Vernon effective action $S_{\text {eff }}\left[g^{ \pm}\right]$in Equation (24) in a form suitable for dimensional regularization. Since both $S_{\mathrm{m}}$ and $S_{\mathrm{g}}$ contain second-order derivatives of the metric, one should also add some boundary terms [206, 361]. The effect of these terms is to cancel out the boundary terms, which appear when taking variations of $S_{\text {eff }}\left[g^{ \pm}\right]$, keeping the value of $g_{a b}^{+}$and $g_{a b}^{-}$ fixed at $\Sigma_{t_{\mathrm{i}}}$ and $\Sigma_{t_{\mathrm{f}}}$. Alternatively, in order to obtain the equations of motion for the metric in the semiclassical regime, we can work with the action terms without boundary terms and neglect all boundary terms when taking variations with respect to $g_{a b}^{ \pm}$. From now on, all the functional derivatives with respect to the metric will be understood in this sense.

The semiclassical Einstein equation (8) can now be derived. Using the definition of the stressenergy tensor $T^{a b}(x)=(2 / \sqrt{-g}) \delta S_{\mathrm{m}} / \delta g_{a b}$ and the definition of the influence functional, Equations (22) and (23), we see that

$$
\left\langle\hat{T}^{a b}[g ; x)\right\rangle=\left.\frac{2}{\sqrt{-g(x)}} \frac{\delta S_{\mathrm{IF}}\left[g^{ \pm}\right]}{\delta g_{a b}^{+}(x)}\right|_{g^{ \pm}=g}
$$


where the expectation value is taken in the $n$-dimensional spacetime generalization of the state described by $\hat{\rho}^{\mathrm{S}}\left(t_{\mathrm{i}}\right)$. Therefore, differentiating $S_{\text {eff }}\left[g^{ \pm}\right]$in Equation (24) with respect to $g_{a b}^{+}$and then setting $g_{a b}^{+}=g_{a b}^{-}=g_{a b}$, we get the semiclassical Einstein equation in $n$ dimensions. This equation is then renormalized by absorbing the divergences in the regularized $\left\langle\hat{T}^{a b}[g]\right\rangle$ into the bare parameters. Taking the limit $n \rightarrow 4$ we obtain the physical semiclassical Einstein equation (8).

\subsection{Influence action for stochastic gravity}

In the spirit of the previous derivation of the Einstein-Langevin equation, we now seek a dynamical equation for a linear perturbation $h_{a b}$ to the semiclassical metric $g_{a b}$, solution of Equation (8). Strictly speaking, if we use dimensional regularization we must consider the $n$-dimensional version of that equation. From the results just described, if such an equation were simply a linearized semiclassical Einstein equation, it could be obtained from an expansion of the effective action $S_{\text {eff }}\left[g+h^{ \pm}\right]$. In particular, since, from Equation (25), we have that

$$
\left\langle\hat{T}^{a b}[g+h ; x)\right\rangle=\left.\frac{2}{\sqrt{-\operatorname{det}(g+h)(x)}} \frac{\delta S_{\mathrm{IF}}\left[g+h^{ \pm}\right]}{\delta h_{a b}^{+}(x)}\right|_{h^{ \pm}=h}
$$

the expansion of $\left\langle\hat{T}^{a b}[g+h]\right\rangle$ to linear order in $h_{a b}$ can be obtained from an expansion of the influence action $S_{\mathrm{IF}}\left[g+h^{ \pm}\right]$up to second order in $h_{a b}^{ \pm}$.

To perform the expansion of the influence action, we have to compute the first and second order functional derivatives of $S_{\mathrm{IF}}\left[g+h^{ \pm}\right]$and then set $h_{a b}^{+}=h_{a b}^{-}=h_{a b}$. If we do so using the path integral representation (22), we can interpret these derivatives as expectation values of operators. The relevant second order derivatives are

$$
\begin{aligned}
& \left.\frac{4}{\sqrt{-g(x)} \sqrt{-g(y)}} \frac{\delta^{2} S_{\mathrm{IF}}\left[g+h^{ \pm}\right]}{\delta h_{a b}^{+}(x) \delta h_{c d}^{+}(y)}\right|_{h^{ \pm}=h}=-H_{\mathrm{S}}^{a b c d}[g ; x, y)-K^{a b c d}[g ; x, y)+i N^{a b c d}[g ; x, y), \\
& \left.\frac{4}{\sqrt{-g(x)} \sqrt{-g(y)}} \frac{\delta^{2} S_{\mathrm{IF}}\left[g^{ \pm}\right]}{\delta h_{a b}^{+}(x) \delta h_{c d}^{-}(y)}\right|_{h^{ \pm}=h}=-H_{\mathrm{A}}^{a b c d}[g ; x, y)-i N^{a b c d}[g ; x, y),
\end{aligned}
$$

where

$$
\begin{aligned}
N^{a b c d}[g ; x, y) & \equiv \frac{1}{2}\left\langle\left\{\hat{t}^{a b}[g ; x), \hat{t}^{c d}[g ; y)\right\}\right\rangle, \\
H_{\mathrm{S}}^{a b c d}[g ; x, y) & \equiv \operatorname{Im}\left\langle\mathrm{T}^{*}\left(\hat{T}^{a b}[g ; x) \hat{T}^{c d}[g ; y)\right)\right\rangle, \\
H_{\mathrm{A}}^{a b c d}[g ; x, y) & \equiv-\frac{i}{2}\left\langle\left[\hat{T}^{a b}[g ; x), \hat{T}^{c d}[g ; y)\right]\right\rangle, \\
K^{a b c d}[g ; x, y) & \equiv \frac{-4}{\sqrt{-g(x)} \sqrt{-g(y)}}\left\langle\left.\frac{\delta^{2} S_{\mathrm{m}}[g+h, \phi]}{\delta h_{a b}(x) \delta h_{c d}(y)}\right|_{\phi=\hat{\phi}}\right\rangle,
\end{aligned}
$$

with $\hat{t}^{a b}$ defined in Equation (13); [, ] denotes the commutator and $\{$,$\} the anti-commutator.$ Here we use a Weyl ordering prescription for the operators. The symbol $\mathrm{T}^{*}$ denotes the following ordered operations: First, time order the field operators $\hat{\phi}$ and then apply the derivative operators, which appear in each term of the product $T^{a b}(x) T^{c d}(y)$, where $T^{a b}$ is the functional (4). This $\mathrm{T}^{*}$ "time ordering" arises because we have path integrals containing products of derivatives of the field, which can be expressed as derivatives of the path integrals, which do not contain such derivatives. Notice, from their definitions, that all the kernels, which appear in expressions (27), are real and also $H_{\mathrm{A}}^{a b c d}$ is free of ultraviolet divergences in the limit as $n \rightarrow 4$. 
From Equations (25) and (25), since $S_{\mathrm{IF}}[g, g]=0$ and $S_{\mathrm{IF}}\left[g^{-}, g^{+}\right]=-S_{\mathrm{IF}}^{*}\left[g^{+}, g^{-}\right]$, we can write the expansion for the influence action $S_{\mathrm{IF}}\left[g+h^{ \pm}\right]$around a background metric $g_{a b}$ in terms of the previous kernels. Taking into account that these kernels satisfy the symmetry relations

$$
H_{\mathrm{S}}^{a b c d}(x, y)=H_{\mathrm{S}}^{c d a b}(y, x), \quad H_{\mathrm{A}}^{a b c d}(x, y)=-H_{\mathrm{A}}^{c d a b}(y, x), \quad K^{a b c d}(x, y)=K^{c d a b}(y, x),
$$

and introducing the new kernel

$$
H^{a b c d}(x, y) \equiv H_{\mathrm{S}}^{a b c d}(x, y)+H_{\mathrm{A}}^{a b c d}(x, y),
$$

the expansion of $S_{\mathrm{IF}}$ can be finally written as

$$
\begin{aligned}
S_{\mathrm{IF}}\left[g+h^{ \pm}\right]= & \frac{1}{2} \int d^{4} x \sqrt{-g(x)}\left\langle\hat{T}^{a b}[g ; x)\right\rangle\left[h_{a b}(x)\right] \\
& -\frac{1}{8} \int d^{4} x d^{4} y \sqrt{-g(x)} \sqrt{-g(y)}\left[h_{a b}(x)\right]\left(H^{a b c d}[g ; x, y)+K^{a b c d}[g ; x, y)\right)\left\{h_{c d}(y)\right\} \\
& +\frac{i}{8} \int d^{4} x d^{4} y \sqrt{-g(x)} \sqrt{-g(y)}\left[h_{a b}(x)\right] N^{a b c d}[g ; x, y)\left[h_{c d}(y)\right]+\mathcal{O}\left(h^{3}\right),
\end{aligned}
$$

where we have used the notation

$$
\left[h_{a b}\right] \equiv h_{a b}^{+}-h_{a b}^{-}, \quad\left\{h_{a b}\right\} \equiv h_{a b}^{+}+h_{a b}^{-} .
$$

From Equations (26) and (30) it is clear that the imaginary part of the influence action does not contribute to the perturbed semiclassical Einstein equation (the expectation value of the stressenergy tensor is real). However, as it depends on the noise kernel, it contains information on the fluctuations of the operator $\hat{T}^{a b}[g]$.

We are now in a position to carry out the derivation of the semiclassical Einstein-Langevin equation. The procedure is well known [38, 58, 73, 132, 206, 308, 379]: It consists of deriving a new "stochastic" effective action from the observation that the effect of the imaginary part of the influence action (30) on the corresponding influence functional is equivalent to the averaged effect of the stochastic source $\xi^{a b}$ coupled linearly to the perturbations $h_{a b}^{ \pm}$. This observation follows from the identity first invoked by Feynman and Vernon for such purpose:

$$
\begin{array}{r}
\exp \left(-\frac{1}{8} \int d^{4} x d^{4} y \sqrt{-g(x)} \sqrt{-g(y)}\left[h_{a b}(x)\right] N^{a b c d}(x, y)\left[h_{c d}(y)\right]\right)= \\
\int \mathcal{D} \xi \mathcal{P}[\xi] \exp \left(\frac{i}{2} \int d^{4} x \sqrt{-g(x)} \xi^{a b}(x)\left[h_{a b}(x)\right]\right),
\end{array}
$$

where $\mathcal{P}[\xi]$ is the probability distribution functional of a Gaussian stochastic tensor $\xi^{a b}$ characterized by the correlators (14) with $N^{a b c d}$ given by Equation (12), and where the path integration measure is assumed to be a scalar under diffeomorphisms of $\left(\mathcal{M}, g_{a b}\right)$. The above identity follows from the identification of the right-hand side of Equation (32) with the characteristic functional for the stochastic field $\xi^{a b}$. The probability distribution functional for $\xi^{a b}$ is explicitly given by

$$
\mathcal{P}[\xi]=\operatorname{det}(2 \pi N)^{-1 / 2} \exp \left[-\frac{1}{2} \int d^{4} x d^{4} y \sqrt{-g(x)} \sqrt{-g(y)} \xi^{a b}(x) N_{a b c d}^{-1}(x, y) \xi^{c d}(y)\right] .
$$

We may now introduce the stochastic effective action as

$$
S_{\mathrm{eff}}^{\mathrm{s}}\left[g+h^{ \pm}, \xi\right] \equiv S_{\mathrm{g}}\left[g+h^{+}\right]-S_{\mathrm{g}}\left[g+h^{-}\right]+S_{\mathrm{IF}}^{\mathrm{s}}\left[g+h^{ \pm}, \xi\right],
$$

where the "stochastic" influence action is defined as

$$
S_{\mathrm{IF}}^{\mathrm{s}}\left[g+h^{ \pm}, \xi\right] \equiv \operatorname{Re} S_{\mathrm{IF}}\left[g+h^{ \pm}\right]+\frac{1}{2} \int d^{4} x \sqrt{-g(x)} \xi^{a b}(x)\left[h_{a b}(x)\right]+\mathcal{O}\left(h^{3}\right) .
$$


Note that, in fact, the influence functional can now be written as a statistical average over $\xi^{a b}$ :

$$
\mathcal{F}_{\mathrm{IF}}\left[g+h^{ \pm}\right]=\left\langle\exp \left(i S_{\mathrm{IF}}^{\mathrm{s}}\left[g+h^{ \pm}, \xi\right]\right)\right\rangle_{\mathrm{s}} .
$$

The stochastic equation of motion for $h_{a b}$ reads

$$
\left.\frac{\delta S_{\mathrm{eff}}^{\mathrm{s}}\left[g+h^{ \pm}, \xi\right]}{\delta h_{a b}^{+}(x)}\right|_{h^{ \pm}=h}=0
$$

which is the Einstein-Langevin equation (15); notice that only the real part of $S_{\mathrm{IF}}$ contributes to the expectation value (26). To be precise, we first get the regularized $n$-dimensional equations with the bare parameters, with the tensor $A^{a b}$ replaced by $\frac{2}{3} D^{a b}$, where

$$
\begin{aligned}
D^{a b} & \equiv \frac{1}{\sqrt{-g}} \frac{\delta}{\delta g_{a b}} \int d^{n} x \sqrt{-g}\left(R_{c d e f} R^{c d e f}-R_{c d} R^{c d}\right) \\
& =\frac{g^{a b}}{2}\left(R_{c d e f} R^{c d e f}-R_{c d} R^{c d}+\square R\right)-2 R^{a c d e} R_{c d e}^{b}-2 R^{a c b d} R_{c d}+4 R^{a c} R_{c}{ }^{b}-3 \square R^{a b}+\nabla^{a} \nabla^{b} R .
\end{aligned}
$$

Of course, when $n=4$, these tensors are related, $A^{a b}=\frac{2}{3} D^{a b}$. After that we renormalize and take the limit $n \rightarrow 4$ to obtain the Einstein-Langevin equations in the physical spacetime.

\subsection{Explicit form of the Einstein-Langevin equation}

We can write the Einstein-Langevin equation in a more explicit form by working out the expansion of $\left\langle\hat{T}^{a b}[g+h]\right\rangle$ up to linear order in the perturbation $h_{a b}$. From Equation (26), we see that this expansion can be easily obtained from Equation (30). The result is

$$
\left\langle\hat{T}_{n}^{a b}[g+h ; x)\right\rangle=\left\langle\hat{T}_{n}^{a b}[g, x)\right\rangle+\left\langle\hat{T}_{n}^{(1) a b}[g, h ; x)\right\rangle-\frac{1}{2} \int d^{n} y \sqrt{-g(y)} H_{n}^{a b c d}[g ; x, y) h_{c d}(y)+\mathcal{O}\left(h^{2}\right) .
$$

Here we use a subscript $n$ on a given tensor to indicate that we are explicitly working in $n$ dimensions, as we use dimensional regularization, and we also use the superindex ${ }^{(1)}$ to generally indicate that the tensor is the first-order correction, linear in $h_{a b}$, in a perturbative expansion around the background $g_{a b}$.

Using the Klein-Gordon equation (2) and expression (4) for the stress-energy tensor and the corresponding operator, we can write

$$
\hat{T}_{n}^{(1) a b}[g, h]=\left(\frac{1}{2} g^{a b} h_{c d}-\delta_{c}^{a} h_{d}^{b}-\delta_{c}^{b} h_{d}^{a}\right) \hat{T}_{n}^{c d}[g]+\mathcal{F}^{a b}[g, h] \hat{\phi}_{n}^{2}[g],
$$

where $\mathcal{F}^{a b}[g ; h]$ is the differential operator

$$
\begin{aligned}
\mathcal{F}^{a b} \equiv & \left(\xi-\frac{1}{4}\right)\left(h^{a b}-\frac{1}{2} g^{a b} h_{c}^{c}\right) \square \\
& +\frac{x i}{2}\left[\nabla^{c} \nabla^{a} h_{c}^{b}+\nabla^{c} \nabla^{b} h_{c}^{a}-\square h^{a b}-\nabla^{a} \nabla^{b} h_{c}^{c}-g^{a b} \nabla^{c} \nabla^{d} h_{c d}+g^{a b} \square h_{c}^{c}\right. \\
& \left.+\left(\nabla^{a} h_{c}^{b}+\nabla^{b} h_{c}^{a}-\nabla_{c} h^{a b}-2 g^{a b} \nabla^{d} h_{c d}+g^{a b} \nabla_{c} h_{d}^{d}\right) \nabla^{c}-g^{a b} h_{c d} \nabla^{c} \nabla^{d}\right] .
\end{aligned}
$$

It is understood that indices are raised with the background inverse metric $g^{a b}$, and that all the covariant derivatives are associated with the metric $g_{a b}$. 
Substituting Equation (38) into the $n$-dimensional version of the Einstein-Langevin Equation (15), taking into account that $g_{a b}$ satisfies the semiclassical Einstein equation (8), and substituting expression (39), we can write the Einstein-Langevin equation in dimensional regularization as

$$
\begin{aligned}
& \frac{1}{8 \pi G_{\mathrm{B}}}\left[G^{(1) a b}-\frac{1}{2} g^{a b} G^{c d} h_{c d}+G^{a c} h_{c}^{b}+G^{b c} h_{c}^{a}+\Lambda_{\mathrm{B}}\left(h^{a b}-\frac{1}{2} g^{a b} h_{c}^{c}\right)\right] \\
& -\frac{4 \alpha_{\mathrm{B}}}{3}\left(D^{(1) a b}-\frac{1}{2} g^{a b} D^{c d} h_{c d}+D^{a c} h_{c}^{b}+D^{b c} h_{c}^{a}\right) \\
& -2 \beta_{\mathrm{B}}\left(B^{(1) a b}-\frac{1}{2} g^{a b} B^{c d} h_{c d}+B^{a c} h_{c}^{b}+B^{b c} h_{c}^{a}\right) \\
& -\mu^{-(n-4)} \mathcal{F}_{x}^{a b}\left\langle\hat{\phi}_{n}^{2}[g ; x)\right\rangle+\frac{1}{2} \int d^{n} y \sqrt{-g(y)} \mu^{-(n-4)} H_{n}^{a b c d}[g ; x, y) h_{c d}(y) \\
= & \mu^{-(n-4)} \xi_{n}^{a b},
\end{aligned}
$$

where the tensors $G^{a b}, D^{a b}$, and $B^{a b}$ are computed from the semiclassical metric $g_{a b}$, and where we have omitted the functional dependence on $g_{a b}$ and $h_{a b}$ in $G^{(1) a b}, D^{(1) a b}, B^{(1) a b}$, and $\mathcal{F}^{a b}$ to simplify the notation. The parameter $\mu$ is a mass scale, which relates the dimensions of the physical field $\phi$ with the dimensions of the corresponding field in $n$ dimensions, $\phi_{n}=\mu^{(n-4) / 2} \phi$. Notice that, in Equation (41), all the ultraviolet divergences in the limit $n \rightarrow 4$, which must be removed by renormalization of the coupling constants, are in $\left\langle\hat{\phi}_{n}^{2}(x)\right\rangle$ and the symmetric part $H_{\mathrm{S}_{n}}^{a b c d}(x, y)$ of the kernel $H_{n}^{a b c d}(x, y)$, whereas the kernels $N_{n}^{a b c d}(x, y)$ and $H_{\mathrm{A}_{n}}^{a b c d}(x, y)$ are free of ultraviolet divergences. If we introduce the bitensor $F_{n}^{a b c d}[g ; x, y)$ defined by

$$
F_{n}^{a b c d}[g ; x, y) \equiv\left\langle\hat{t}_{n}^{a b}[g ; x) \hat{t}_{n}^{\rho \sigma}[g ; y)\right\rangle,
$$

where $\hat{t}^{a b}$ is defined by Equation (13), then the kernels $N$ and $H_{\mathrm{A}}$ can be written as

$$
N_{n}^{a b c d}[g ; x, y)=\operatorname{Re} F_{n}^{a b c d}[g ; x, y), \quad H_{\mathrm{A}_{n}}^{a b c d}[g ; x, y)=\operatorname{Im} F_{n}^{a b c d}[g ; x, y),
$$

where we have used

$$
2\left\langle\hat{t}^{a b}(x) \hat{t}^{c d}(y)\right\rangle=\left\langle\left\{\hat{t}^{a b}(x), \hat{t}^{c d}(y)\right\}\right\rangle+\left\langle\left[\hat{t}^{a b}(x), \hat{t}^{c d}(y)\right]\right\rangle,
$$

and the fact that the first term on the right-hand side of this identity is real, whereas the second one is purely imaginary. Once we perform the renormalization procedure in Equation (41), setting $n=4$ will yield the physical Einstein-Langevin equation. Due to the presence of the kernel $H_{n}^{a b c d}(x, y)$, this equation will usually be non-local in the metric perturbation. In Section 6 we will carry out an explicit evaluation of the physical Einstein-Langevin equation, which will illustrate the procedure.

\subsubsection{The kernels for the vacuum state}

When the expectation values in the Einstein-Langevin equation are taken in a vacuum state $|0\rangle$, such as, an "in" vacuum, we can be more explicit, since we can write the expectation values in terms of the Wightman and Feynman functions, defined as

$$
G_{n}^{+}[g ; x, y) \equiv\left\langle 0\left|\hat{\phi}_{n}[g ; x) \hat{\phi}_{n}[g ; y)\right| 0\right\rangle, \quad i G_{F_{n}}[g ; x, y) \equiv\left\langle 0\left|\mathrm{~T}\left(\hat{\phi}_{n}[g ; x) \hat{\phi}_{n}[g ; y)\right)\right| 0\right\rangle .
$$

These expressions for the kernels in the Einstein-Langevin equation will be very useful for explicit calculations. To simplify the notation, we omit the functional dependence on the semiclassical metric $g_{a b}$, which will be understood in all the expressions below. 
From Equations (43), we see that the kernels $N_{n}^{a b c d}(x, y)$ and $H_{\mathrm{A}_{n}}^{a b c d}(x, y)$ are the real and imaginary parts, respectively, of the bitensor $F_{n}^{a b c d}(x, y)$. From expression (5) we see that the stress-energy operator $\hat{T}_{n}^{a b}$ can be written as a sum of terms of the form $\left\{\mathcal{A}_{x} \hat{\phi}_{n}(x), \mathcal{B}_{x} \hat{\phi}_{n}(x)\right\}$, where $\mathcal{A}_{x}$ and $\mathcal{B}_{x}$ are differential operators. It then follows that we can express the bitensor $F_{n}^{a b c d}(x, y)$ in terms of the Wightman function as

$$
\begin{aligned}
F_{n}^{a b c d}(x, y)= & \nabla_{x}^{a} \nabla_{y}^{c} G_{n}^{+}(x, y) \nabla_{x}^{b} \nabla_{y}^{d} G_{n}^{+}(x, y)+\nabla_{x}^{a} \nabla_{y}^{d} G_{n}^{+}(x, y) \nabla_{x}^{b} \nabla_{y}^{c} G_{n}^{+}(x, y) \\
& +2 \mathcal{D}_{x}^{a b}\left(\nabla_{y}^{c} G_{n}^{+}(x, y) \nabla_{y}^{d} G_{n}^{+}(x, y)\right)+2 \mathcal{D}_{y}^{c d}\left(\nabla_{x}^{a} G_{n}^{+}(x, y) \nabla_{x}^{b} G_{n}^{+}(x, y)\right) \\
& +2 \mathcal{D}_{x}^{a b} \mathcal{D}_{y}^{c d}\left(G_{n}^{+2}(x, y)\right),
\end{aligned}
$$

where $\mathcal{D}_{x}^{a b}$ is the differential operator (6). From this expression and relations (43), we get expressions for the kernels $N_{n}$ and $H_{\mathrm{A}_{n}}$ in terms of the Wightman function $G_{n}^{+}(x, y)$.

Similarly, the kernel $H_{\mathrm{S}_{n}}^{a b c d}(x, y)$ can be written in terms of the Feynman function as

$$
\begin{aligned}
H_{\mathrm{S}_{n}}^{a b c d}(x, y)=-\operatorname{Im}[ & \nabla_{x}^{a} \nabla_{y}^{c} G_{F_{n}}(x, y) \nabla_{x}^{b} \nabla_{y}^{d} G_{F_{n}}(x, y)+\nabla_{x}^{a} \nabla_{y}^{d} G_{F_{n}}(x, y) \nabla_{x}^{b} \nabla_{y}^{c} G_{F_{n}}(x, y) \\
& -g^{a b}(x) \nabla_{x}^{e} \nabla_{y}^{c} G_{F_{n}}(x, y) \nabla_{e}^{x} \nabla_{y}^{d} G_{F_{n}}(x, y) \\
& -g^{c d}(y) \nabla_{x}^{a} \nabla_{y}^{e} G_{F_{n}}(x, y) \nabla_{x}^{b} \nabla_{e}^{y} G_{F_{n}}(x, y) \\
& +\frac{1}{2} g^{a b}(x) g^{c d}(y) \nabla_{x}^{e} \nabla_{y}^{f} G_{F_{n}}(x, y) \nabla_{e}^{x} \nabla_{f}^{y} G_{F_{n}}(x, y) \\
& +\mathcal{K}_{x}^{a b}\left(2 \nabla_{y}^{c} G_{F_{n}}(x, y) \nabla_{y}^{d} G_{F_{n}}(x, y)-g^{c d}(y) \nabla_{y}^{e} G_{F_{n}}(x, y) \nabla_{e}^{y} G_{F_{n}}(x, y)\right) \\
& +\mathcal{K}_{y}^{c d}\left(2 \nabla_{x}^{a} G_{F_{n}}(x, y) \nabla_{x}^{b} G_{F_{n}}(x, y)-g^{a b}(x) \nabla_{x}^{e} G_{F_{n}}(x, y) \nabla_{e}^{x} G_{F_{n}}(x, y)\right) \\
& \left.+2 \mathcal{K}_{x}^{a b} \mathcal{K}_{y}^{c d}\left(G_{F_{n}}^{2}(x, y)\right)\right],
\end{aligned}
$$

where $\mathcal{K}_{x}^{a b}$ is the differential operator

$$
\mathcal{K}_{x}^{a b} \equiv \xi\left(g^{a b}(x) \square_{x}-\nabla_{x}^{a} \nabla_{x}^{b}+G^{a b}(x)\right)-\frac{1}{2} m^{2} g^{a b}(x) .
$$

Note that, in the vacuum state $|0\rangle$, the term $\left\langle\hat{\phi}_{n}^{2}(x)\right\rangle$ in Equation (41) can also be written as $\left\langle\hat{\phi}_{n}^{2}(x)\right\rangle=i G_{F_{n}}(x, x)=G_{n}^{+}(x, x)$.

Finally, the causality of the Einstein-Langevin equation (41) can be explicitly seen as follows. The non-local terms in that equation are due to the kernel $H(x, y)$, which is defined in Equation (29) as the sum of $H_{\mathrm{S}}(x, y)$ and $H_{\mathrm{A}}(x, y)$. Now, when the points $x$ and $y$ are spacelike separated, $\hat{\phi}_{n}(x)$ and $\hat{\phi}_{n}(y)$ commute and, thus, $G_{n}^{+}(x, y)=i G_{F_{n}}(x, y)=\frac{1}{2}\left\langle 0\left|\left\{\hat{\phi}_{n}(x), \hat{\phi}_{n}(y)\right\}\right| 0\right\rangle$, which is real. Hence, from the above expressions, we have that $H_{\mathrm{A}_{n}}^{a b c d}(x, y)=H_{\mathrm{S}_{n}}^{a b c d}(x, y)=0$, and thus $H_{n}^{a b c d}(x, y)=0$. This fact is expected since, from the causality of the expectation value of the stress-energy operator [359], we know that the non-local dependence on the metric perturbation in the Einstein-Langevin equation, see Equation (15), must be causal. See [208] for an alternative proof of the causal nature of the Einstein-Langevin equation. 


\section{$5 \quad$ Noise Kernel and Point Separation}

In this section we explore further the properties of the noise kernel and the stress-energy bitensor. Similar to what was done for the stress-energy tensor, it is desirable to relate the noise kernel defined at separated points to the Green's function of a quantum field. We pointed out earlier [187] that field quantities defined at two separated points may possess important information, which could be the starting point for probes into possible extended structures of spacetime. Of more practical concern is how one can define a finite quantity at one point or in some small region around it from the noise kernel defined at two separated points. When we refer to, say, the fluctuations of energy density in ordinary (point-wise) quantum field theory, we are in actuality asking such a question. This is essential for addressing fundamental issues like

- the validity of semiclassical gravity [237], e.g., whether the fluctuations to mean ratio is a correct criterion [10, 11, 113, 115, 198, 303];

- whether the fluctuations in the vacuum energy density, which drive some models of inflationary cosmology, violate the positive-energy condition;

- the physical effects of black-hole horizon fluctuations and Hawking radiation backreaction to begin with, are the fluctuations finite or infinite?

- general relativity as a low-energy effective theory in the geometro-hydrodynamic limit towards a kinetic-theory approach to quantum gravity [185, 187, 188].

Thus, for comparison with ordinary phenomena at low energy, we need to find a reasonable prescription for obtaining a finite quantity of the noise kernel in the limit of ordinary (point-defined) quantum field theory. It is well-known that several regularization methods can work equally well for the removal of ultraviolet divergences in the stress-energy tensor of quantum fields in curved spacetime. Their mutual relations are known and discrepancies explained. This formal structure of regularization schemes for quantum fields in curved spacetime should remain intact when applied to the regularization of the noise kernel in general curved spacetimes; it is the meaning and relevance of regularization of the noise kernel, which is of more concern (see comments below). Specific considerations will, of course, enter for each method. But for the methods employed so far (such as zeta-function, point separation, dimensional and smeared-field) applied to simple cases (Casimir, Einstein, thermal fields) there is no new inconsistency or discrepancy.

Regularization schemes used in obtaining a finite expression for the stress-energy tensor have been applied to the noise kernel. This includes the simple normal-ordering [237, 378] and smearedfield operator [303] methods applied to the Minkowski and Casimir spaces, zeta-function [68, 106, 232] for spacetimes with an Euclidean section, applied to the Casimir effect [86] and the Einstein universe [302], or the covariant point-separation methods applied to the Minkowski [303], hot flat space and Schwarzschild spacetime [305]. There are differences and deliberations on whether it is meaningful to seek a point-wise expression for the noise kernel, and if so what is the correct way to proceed, e.g., regularization by a subtraction scheme or by integrating over a test field. Intuitively the smear-field method [303] may better preserve the integrity of the noise kernel, as it provides a sampling of the two-point function rather than using a subtraction scheme, which alters its innate properties by forcing a nonlocal quantity into a local one. More investigation is needed to clarify these points, which bear on important issues like the validity of semiclassical gravity. We shall set a more modest goal here, to derive a general expression for the noise kernel for quantum fields in an arbitrary curved spacetime in terms of Green's functions and leave the discussion of point-wise limit to a later date. For this purpose the covariant point-separation method that highlights the bitensor features, when used not as a regularization scheme, is perhaps closest to the spirit of stochastic gravity. 
The task of finding a general expression for the noise-kernel for quantum fields in curved spacetimes was carried out by Phillips and $\mathrm{Hu}$ in two papers using the "modified" point-separation scheme [1, 358, 360]. Their first paper [304] begins with a discussion of the procedures for dealing with the quantum stress-tensor bi-operator at two separated points, and ends with a general expression for the noise kernel, defined at separated points expressed as products of covariant derivatives up to the fourth order of the quantum field's Green's function. (The stress tensor involves up to two covariant derivatives.) This result holds for $x \neq y$ without recourse to renormalization of the Green's function, showing that $N_{a b c^{\prime} d^{\prime}}(x, y)$ is always finite for $x \neq y$ (and off the light cone for massless theories). In particular, for a massless conformally-coupled free scalar field on a fourdimensional manifold, they computed the trace of the noise kernel at both points and found this double trace vanishes identically. This implies that there is no stochastic correction to the trace anomaly for massless conformal fields, in agreement with results arrived at in [58, 73, 258] (see also Section 3). In their second paper [305] a Gaussian approximation for the Green's function (which is what limits the accuracy of the results) is used to derive finite expressions for two specific classes of spacetimes, ultrastatic spacetimes, such as the hot flat space, and the conformally- ultrastatic spacetimes, such as the Schwarzschild spacetime. Again, the validity of these results may depend on how we view the relevance and meaning of regularization. We will only report the result of their first paper here.

\subsection{Point separation}

The point separation scheme introduced in the 1960s by DeWitt [92] was brought to more popular use in the 1970s in the context of quantum field theory in curved spacetimes [83, 84, 93] as a means for obtaining a finite quantum stress tensor. Since the stress-energy tensor is built from the product of a pair of field operators evaluated at a single point, it is not well-defined. In this scheme, one introduces an artificial separation of the single point $x$ into a pair of closely separated points $x$ and $x^{\prime}$. The problematic terms involving field products such as $\hat{\phi}(x)^{2}$ become $\hat{\phi}(x) \hat{\phi}\left(x^{\prime}\right)$, whose expectation value is well-defined. If one is interested in the low-energy behavior captured by the point-defined quantum field theory - as the effort in the 1970s was directed one takes the coincidence limit. Once the divergences present are identified, they may be removed (regularization) or moved (by renormalizing the coupling constants), to produce a well-defined, finite stress tensor at a single point.

Thus, the first order of business is the construction of the stress tensor and then to derive the symmetric stress-energy tensor two-point function, the noise kernel, in terms of the Wightman Green's function. In this section we will use the traditional notation for index tensors in the point-separation context.

\subsection{1 n-tensors and end-point expansions}

An object like the Green's function $G(x, y)$ is an example of a bi-scalar; it transforms as a scalar at both points $x$ and $y$. We can also define a bitensor $T_{a_{1} \ldots a_{n} b_{1}^{\prime} \ldots b_{m}^{\prime}}(x, y)$ : Upon a coordinate transformation, this transforms as a rank $n$ tensor at $x$ and a rank $m$ tensor at $y$. We will extend

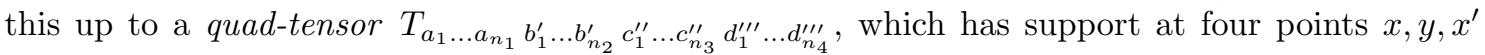
and $y^{\prime}$, transforming as rank $n_{1}, n_{2}, n_{3}$ and $n_{4}$ tensors at each of the four points. This also sets the notation we will use: unprimed indices refer to the tangent space constructed above $x$, single primed indices to $y$, double primed to $x^{\prime}$ and triple primed to $y^{\prime}$. For each point, there is the covariant derivative $\nabla_{a}$ at that point. Covariant derivatives at different points commute, and the covariant derivative at, say, point $x^{\prime}$ does not act on a bitensor defined at, say, $x$ and $y$ :

$$
T_{a b^{\prime} ; c ; d^{\prime}}=T_{a b^{\prime} ; d^{\prime} ; c} \quad \text { and } \quad T_{a b^{\prime} ; c^{\prime \prime}}=0 .
$$


To simplify notation, we will henceforth eliminate the semicolons after the first one for multiple covariant derivatives at multiple points.

Having objects defined at different points, the coincident limit is defined as evaluation "on the diagonal", in the sense of the spacetime support of the function or tensor, and the usual shorthand $[G(x, y)] \equiv G(x, x)$ is used. This extends to $n$-tensors as

$$
\left[T_{a_{1} \ldots a_{n_{1}} b_{1}^{\prime} \ldots b_{n_{2}}^{\prime} c_{1}^{\prime \prime} \ldots c_{n_{3}}^{\prime \prime} d_{1}^{\prime \prime \prime} \ldots d_{n_{4}}^{\prime \prime \prime}}\right]=T_{a_{1} \ldots a_{n_{1}} b_{1} \ldots b_{n_{2}} c_{1} \ldots c_{n_{3}} d_{1} \ldots d_{n_{4}}}
$$

i.e., this becomes a rank $\left(n_{1}+n_{2}+n_{3}+n_{4}\right)$ tensor at $x$. The multi-variable chain rule relates covariant derivatives acting at different points, when we are interested in the coincident limit:

$$
\left[T_{\left.a_{1} \ldots a_{m} b_{1}^{\prime} \ldots b_{n}^{\prime}\right] ; c}=\left[T_{a_{1} \ldots a_{m} b_{1}^{\prime} \ldots b_{n}^{\prime} ; c}\right]+\left[T_{a_{1} \ldots a_{m} b_{1}^{\prime} \ldots b_{n}^{\prime} ; c^{\prime}}\right]\right.
$$

This result is referred to as Synge's theorem in this context; we follow Fulling's discussion [121].

The bitensor of parallel transport $g_{a}{ }^{b^{\prime}}$ is defined such that when it acts on a vector $v_{b^{\prime}}$ at $y$, it parallel transports the vector along the geodesics connecting $x$ and $y$. This allows us to add vectors and tensors defined at different points. We cannot directly add a vector $v_{a}$ at $x$ and vector $w_{a^{\prime}}$ at $y$. But by using $g_{a}{ }^{b^{\prime}}$, we can construct the sum $v^{a}+g_{a}{ }^{b^{\prime}} w_{b^{\prime}}$. We will also need the obvious property $\left[g_{a}{ }^{b^{\prime}}\right]=g_{a}{ }^{b}$.

The main bi-scalar we need is the world function $\sigma(x, y)$. This is defined as a half of the square of the geodesic distance between the points $x$ and $y$. It satisfies the equation

$$
\sigma=\frac{1}{2} \sigma^{; p} \sigma_{; p}
$$

Often in the literature, a covariant derivative is implied when the world function appears with indices, $\sigma^{a} \equiv \sigma^{; a}$, i.e., taking the covariant derivative at $x$, while $\sigma^{a^{\prime}}$ means the covariant derivative at $y$. This is done since the vector $-\sigma^{a}$ is the tangent vector to the geodesic with length equal to the distance between $x$ and $y$. As $\sigma^{a}$ records information about distance and direction for the two points, this makes it ideal for constructing a series expansion of a bi-scalar. The end point expansion of a bi-scalar $S(x, y)$ is of the form

$$
S(x, y)=A^{(0)}+\sigma^{p} A_{p}^{(1)}+\sigma^{p} \sigma^{q} A_{p q}^{(2)}+\sigma^{p} \sigma^{q} \sigma^{r} A_{p q r}^{(3)}+\sigma^{p} \sigma^{q} \sigma^{r} \sigma^{s} A_{p q r s}^{(4)}+\ldots,
$$

where, following our convention, the expansion tensors $A_{a_{1} \ldots a_{n}}^{(n)}$ with unprimed indices have support at $x$ (hence the name end point expansion). Only the symmetric part of these tensors contribute to the expansion. For the purposes of multiplying series expansions it is convenient to separate the distance dependence from the direction dependence. This is done by introducing the unit vector $p^{a}=\sigma^{a} / \sqrt{2 \sigma}$. Then the series expansion can be written

$$
S(x, y)=A^{(0)}+\sigma^{\frac{1}{2}} A^{(1)}+\sigma A^{(2)}+\sigma^{\frac{3}{2}} A^{(3)}+\sigma^{2} A^{(4)}+\ldots
$$

The expansion scalars are related, via $A^{(n)}=2^{n / 2} A_{p_{1} \ldots p_{n}}^{(n)} p^{p_{1}} \ldots p^{p_{n}}$, to the expansion tensors.

The last object we need is the VanVleck-Morette determinant $D(x, y)$, defined as $D(x, y) \equiv$ $-\operatorname{det}\left(-\sigma_{; a b^{\prime}}\right)$. The related bi-scalar

$$
\Delta^{1 / 2}=\left(\frac{D(x, y)}{\sqrt{g(x) g(y)}}\right)^{\frac{1}{2}}
$$

satisfies the equation

$$
\Delta^{1 / 2}\left(4-\sigma_{; p}^{p}\right)-2 \Delta^{1 / 2}{ }_{; p} \sigma^{; p}=0
$$


with the boundary condition $\left[\Delta^{1 / 2}\right]=1$.

Further details on these objects and discussions of the definitions and properties are contained in $[83,84]$ and $[90,301]$. There it is shown how the defining equations for $\sigma$ and $\Delta^{1 / 2}$ are used to determine the coincident limit expression for the various covariant derivatives of the world function $\left(\left[\sigma_{; a}\right],\left[\sigma_{; a b}\right]\right.$, etc.) and how the defining differential equation for $\Delta^{1 / 2}$ can be used to determine the series expansion of $\Delta^{1 / 2}$. We show how the expansion tensors $A_{a_{1} \ldots a_{n}}^{(n)}$ are determined in terms of the coincident limits of covariant derivatives of the bi-scalar $S(x, y)$. ([301] details how point separation can be implemented on the computer to provide easy access to a wider range of applications involving higher derivatives of the curvature tensors.)

\subsection{Stress-energy bitensor operator and noise kernel}

Even though we believe that the point-separated results are more basic in the sense that it reflects a deeper structure of the quantum theory of spacetime, we will nevertheless start with quantities defined at one point, because they are what enter in conventional quantum field theory. We will use point separation to introduce the bi-quantities. The key issue here is thus the distinction between point-defined ( $p t)$ and point-separated (bi) quantities.

For a free classical scalar field $\phi$ with the action $S_{\mathrm{m}}[g, \phi]$ defined in Equation (1), the classical stress-energy tensor is

$$
\begin{aligned}
T_{a b}= & (1-2 \xi) \phi_{; a} \phi_{; b}+\left(2 \xi-\frac{1}{2}\right) \phi_{; p} \phi^{p p} g_{a b}+2 \xi \phi\left(\phi_{; p}^{p}-\phi_{; a b} g_{a b}\right) \\
& +\phi^{2} \xi\left(R_{a b}-\frac{1}{2} R g_{a b}\right)-\frac{1}{2} m^{2} \phi^{2} g_{a b},
\end{aligned}
$$

which is equivalent to the tensor of Equation (4), but written in a slightly different form for convenience. When we make the transition to quantum field theory, we promote the field $\phi(x)$ to a field operator $\hat{\phi}(x)$. The fundamental problem of defining a quantum operator for the stress tensor is immediately visible; the field operator appears quadratically. Since $\hat{\phi}(x)$ is an operator-valued distribution, products at a single point are not well-defined. But if the product is point separated, $\hat{\phi}^{2}(x) \rightarrow \hat{\phi}(x) \hat{\phi}\left(x^{\prime}\right)$, they are finite and well-defined.

Let us first seek a point-separated extension of these classical quantities and then consider the quantum field operators. Point separation is symmetrically extended to products of covariant derivatives of the field according to

$$
\begin{aligned}
\left(\phi_{; a}\right)\left(\phi_{; b}\right) & \rightarrow \frac{1}{2}\left(g_{a}{ }^{p^{\prime}} \nabla_{p^{\prime}} \nabla_{b}+g_{b}{ }^{p^{\prime}} \nabla_{a} \nabla_{p^{\prime}}\right) \phi(x) \phi\left(x^{\prime}\right), \\
\phi\left(\phi_{; a b}\right) & \rightarrow \frac{1}{2}\left(\nabla_{a} \nabla_{b}+g_{a}{ }^{p^{\prime}} g_{b}{ }^{q^{\prime}} \nabla_{p^{\prime}} \nabla_{q^{\prime}}\right) \phi(x) \phi\left(x^{\prime}\right) .
\end{aligned}
$$

The bi-vector of parallel displacement $g_{a}{ }^{a^{\prime}}\left(x, x^{\prime}\right)$ is included so that we may have objects that are rank two tensors at $x$ and scalars at $x^{\prime}$.

To carry out point separation on Equation (56), we first define the differential operator

$$
\begin{aligned}
\mathcal{T}_{a b}= & \frac{1}{2}(1-2 \xi)\left(g_{a}{ }^{a^{\prime}} \nabla_{a^{\prime}} \nabla_{b}+g_{b}{ }^{b^{\prime}} \nabla_{a} \nabla_{b^{\prime}}\right)+\left(2 \xi-\frac{1}{2}\right) g_{a b} g^{c d^{\prime}} \nabla_{c} \nabla_{d^{\prime}} \\
& -\xi\left(\nabla_{a} \nabla_{b}+g_{a}{ }^{a^{\prime}} g_{b}{ }^{b^{\prime}} \nabla_{a^{\prime}} \nabla_{b^{\prime}}\right)+\xi g_{a b}\left(\nabla_{c} \nabla^{c}+\nabla_{c^{\prime}} \nabla^{c^{\prime}}\right)+\xi\left(R_{a b}-\frac{1}{2} g_{a b} R\right)-\frac{1}{2} m^{2} g_{a b},
\end{aligned}
$$

from which we obtain the classical stress tensor as

$$
T_{a b}(x)=\lim _{x^{\prime} \rightarrow x} \mathcal{T}_{a b} \phi(x) \phi\left(x^{\prime}\right) .
$$


That the classical tensor field no longer appears as a product of scalar fields at a single point allows a smooth transition to the quantum tensor field. From the viewpoint of the stress tensor, the separation of points is an artificial construct, so when promoting the classical field to a quantum one, neither point should be favored. The product of field configurations is taken to be the symmetrized operator product, denoted by curly brackets:

$$
\phi(x) \phi(y) \rightarrow \frac{1}{2}\{\hat{\phi}(x), \hat{\phi}(y)\}=\frac{1}{2}(\hat{\phi}(x) \hat{\phi}(y)+\hat{\phi}(y) \hat{\phi}(x))
$$

With this, the point separated stress-energy tensor operator is defined as

$$
\hat{T}_{a b}\left(x, x^{\prime}\right) \equiv \frac{1}{2} \mathcal{T}_{a b}\left\{\hat{\phi}(x), \hat{\phi}\left(x^{\prime}\right)\right\}
$$

While the classical stress tensor was defined at the coincidence limit $x^{\prime} \rightarrow x$, we cannot attach any physical meaning to the quantum stress tensor at one point until the issue of regularization is dealt with, which will happen in the next section. For now, we will maintain point separation so as to have a mathematically meaningful operator.

The expectation value of the point-separated stress tensor can now be taken. This amounts to replacing the field operators by their expectation values, which is given by the Hadamard (or Schwinger) function

$$
G^{(1)}\left(x, x^{\prime}\right)=\left\langle\left\{\hat{\phi}(x), \hat{\phi}\left(x^{\prime}\right)\right\}\right\rangle,
$$

and the point-separated stress tensor is defined as

$$
\left\langle\hat{T}_{a b}\left(x, x^{\prime}\right)\right\rangle=\frac{1}{2} \mathcal{T}_{a b} G^{(1)}\left(x, x^{\prime}\right),
$$

where, since $\mathcal{T}_{a b}$ is a differential operator, it can be taken "outside" the expectation value. The expectation value of the point-separated quantum stress tensor for a free, massless $(m=0)$ conformally-coupled $\left(\xi=\frac{1}{6}\right)$ scalar field on a four-dimensional spacetime with scalar curvature $R$ is

$$
\begin{aligned}
\left\langle\hat{T}_{a b}\left(x, x^{\prime}\right)\right\rangle= & \frac{1}{6}\left(g^{p^{\prime}}{ }_{b} G_{; p^{\prime} a}^{(1)}+g^{p^{\prime}}{ }_{a} G^{(1)} ; p^{\prime} b\right)-\frac{1}{12} g^{p^{\prime}}{ }_{q} G_{; p^{\prime}}^{(1)} g_{a b} \\
& -\frac{1}{12}\left(g^{p^{\prime}}{ }_{a} g^{q^{\prime}}{ }_{b} G^{(1)} p_{p^{\prime} q^{\prime}}+G_{; a b}^{(1)}\right)+\frac{1}{12}\left[\left(G_{; p^{\prime}}^{(1)}+G_{; p}^{(1)} p^{p}\right) g_{a b}\right] \\
& +\frac{1}{12} G^{(1)}\left(R_{a b}-\frac{1}{2} R g_{a b}\right) .
\end{aligned}
$$

\subsubsection{Finiteness of the noise kernel}

We now turn our attention to the noise kernel introduced in Equation (12), which is the symmetrized product of the (mean subtracted) stress-tensor operator:

$$
\begin{aligned}
8 N_{a b, c^{\prime} d^{\prime}}(x, y) & =\left\langle\left\{\hat{T}_{a b}(x)-\left\langle\hat{T}_{a b}(x)\right\rangle, \hat{T}_{c^{\prime} d^{\prime}}(y)-\left\langle\hat{T}_{c^{\prime} d^{\prime}}(y)\right\rangle\right\}\right\rangle \\
& =\left\langle\left\{\hat{T}_{a b}(x), \hat{T}_{c^{\prime} d^{\prime}}(y)\right\}\right\rangle-2\left\langle\hat{T}_{a b}(x)\right\rangle\left\langle\hat{T}_{c^{\prime} d^{\prime}}(y)\right\rangle .
\end{aligned}
$$

Since $\hat{T}_{a b}(x)$ defined at one point can be ill-behaved, as it is generally divergent, one can question the soundness of these quantities. But as will be shown later, the noise kernel is finite for $y \neq x$. All field-operator products present in the first expectation value that could be divergent, are canceled by similar products in the second term. We will replace each of the stress-tensor operators in 
the above expression for the noise kernel by their point-separated versions, effectively separating the two points $(x, y)$ into the four points $\left(x, x^{\prime}, y, y^{\prime}\right)$. This will allow us to express the noise kernel in terms of a pair of differential operators acting on a combination of four and two-point functions. Wick's theorem will allow the four-point functions to be re-expressed in terms of twopoint functions. From this we see that all possible divergences for $y \neq x$ will cancel. When the coincidence limit is taken, divergences do occur. The above procedure will allow us to isolate the divergences and to obtain a finite result.

Taking the point-separated quantities as more basic, one should replace each of the stress-tensor operators in the above with the corresponding point-separated version (60), with $\mathcal{T}_{a b}$ acting at $x$ and $x^{\prime}$ and $\mathcal{T}_{c^{\prime} d^{\prime}}$ acting at $y$ and $y^{\prime}$. In this framework the noise kernel is defined as

$$
8 N_{a b, c^{\prime} d^{\prime}}(x, y)=\lim _{x^{\prime} \rightarrow x} \lim _{y^{\prime} \rightarrow y} \mathcal{T}_{a b} \mathcal{T}_{c^{\prime} d^{\prime}} G\left(x, x^{\prime}, y, y^{\prime}\right),
$$

where the four-point function is

$$
G\left(x, x^{\prime}, y, y^{\prime}\right)=\frac{1}{4}\left[\left\langle\left\{\left\{\hat{\phi}(x), \hat{\phi}\left(x^{\prime}\right)\right\},\left\{\hat{\phi}(y), \hat{\phi}\left(y^{\prime}\right)\right\}\right\}\right\rangle-2\left\langle\left\{\hat{\phi}(x), \hat{\phi}\left(x^{\prime}\right)\right\}\right\rangle\left\langle\left\{\hat{\phi}(y), \hat{\phi}\left(y^{\prime}\right)\right\}\right\rangle\right] .
$$

We assume that the pairs $\left(x, x^{\prime}\right)$ and $\left(y, y^{\prime}\right)$ are each within their respective Riemann normalcoordinate neighborhoods so as to avoid the problem that possible geodesic caustics might be present. When we later turn our attention to computing the limit $y \rightarrow x$, after issues of regularization are addressed, we will want to assume that all four points are within the same Riemann normal-coordinate neighborhood.

Wick's theorem, for the case of the free fields that we are considering, gives the simple product four-point function in terms of a sum of products of Wightman functions (we use the shorthand notation $\left.G_{x y} \equiv G_{+}(x, y)=\langle\hat{\phi}(x) \hat{\phi}(y)\rangle\right)$ :

$$
\left\langle\hat{\phi}(x) \hat{\phi}(y) \hat{\phi}\left(x^{\prime}\right) \hat{\phi}\left(y^{\prime}\right)\right\rangle=G_{x y^{\prime}} G_{y x^{\prime}}+G_{x x^{\prime}} G_{y y^{\prime}}+G_{x y} G_{x^{\prime} y^{\prime}} .
$$

Expanding out the anti-commutators in Equation (66) and applying Wick's theorem, the four-point function becomes

$$
G\left(x, x^{\prime}, y, y^{\prime}\right)=G_{x y^{\prime}} G_{x^{\prime} y}+G_{x y} G_{x^{\prime} y^{\prime}}+G_{y x^{\prime}} G_{y^{\prime} x}+G_{y x} G_{y^{\prime} x^{\prime}} .
$$

We can now easily see that the noise kernel defined via this function is indeed well-defined for the limit $\left(x^{\prime}, y^{\prime}\right) \rightarrow(x, y)$ :

$$
G(x, x, y, y)=2\left(G_{x y}^{2}+G_{y x}^{2}\right) .
$$

From this we can see that the noise kernel is also well-defined for $y \neq x$; any divergence present in the first expectation value of Equation (66) has been cancelled by those present in the pair of Green's functions in the second term, in agreement with the results of Section 3.

\subsubsection{Explicit form of the noise kernel}

We will keep the points separated for a while so we can keep track of which covariant derivative acts on which arguments of which Wightman function. As an example (the complete calculation is quite long), consider the result of the first set of covariant derivative operators in the differential operator (57), from both $\mathcal{T}_{a b}$ and $\mathcal{T}_{c^{\prime} d^{\prime}}$, acting on $G\left(x, x^{\prime}, y, y^{\prime}\right)$ :

$$
\frac{1}{4}(1-2 \xi)^{2}\left(g_{a}{ }^{p^{\prime \prime}} \nabla_{p^{\prime \prime}} \nabla_{b}+g_{b}{ }^{p^{\prime \prime}} \nabla_{p^{\prime \prime}} \nabla_{a}\right)\left(g_{c^{\prime}}{ }^{\prime \prime \prime \prime} \nabla_{q^{\prime \prime \prime}} \nabla_{d^{\prime}}+g_{d^{\prime}}{ }^{\prime \prime \prime \prime} \nabla_{q^{\prime \prime \prime}} \nabla_{c^{\prime}}\right) G\left(x, x^{\prime}, y, y^{\prime}\right) .
$$

(Our notation is that $\nabla_{a}$ acts at $x, \nabla_{c^{\prime}}$ at $y, \nabla_{b^{\prime \prime}}$ at $x^{\prime}$, and $\nabla_{d^{\prime \prime \prime}}$ at $y^{\prime}$.) Expanding out the differential operator above, we can determine which derivatives act on which Wightman function: 


$$
\begin{aligned}
& \frac{(1-2 \xi)^{2}}{4} \times\left[g_{c^{\prime}} p^{p^{\prime \prime \prime}} g^{q^{\prime \prime}}{ }_{a}\right.\left(G_{x y^{\prime} ; b p^{\prime \prime \prime}} G_{x^{\prime} y ; q^{\prime \prime} d^{\prime}}+G_{x y ; b d^{\prime}} G_{x^{\prime} y^{\prime} ; q^{\prime \prime} p^{\prime \prime \prime}}\right. \\
&\left.+G_{y x^{\prime} ; q^{\prime \prime} d^{\prime}} G_{y^{\prime} x ; b p^{\prime \prime \prime}}+G_{y x ; b d^{\prime}} G_{y^{\prime} x^{\prime} ; q^{\prime \prime} p^{\prime \prime \prime}}\right) \\
&+g_{d^{\prime}} p^{\prime \prime \prime} g^{q^{\prime \prime}}{ }_{a}\left(G_{x y^{\prime} ; b p^{\prime \prime \prime}} G_{x^{\prime} y ; q^{\prime \prime} c^{\prime}}+G_{x y ; b c^{\prime}} G_{x^{\prime} y^{\prime} ; q^{\prime \prime} p^{\prime \prime \prime}}\right. \\
&\left.+G_{y x^{\prime} ; q^{\prime \prime} c^{\prime}} G_{y^{\prime} x ; b p^{\prime \prime \prime}}+G_{y x ; b c^{\prime}} G_{y^{\prime} x^{\prime} ; q^{\prime \prime} p^{\prime \prime \prime}}\right) \\
&+g_{c^{\prime}} p^{\prime \prime \prime \prime} g^{q^{\prime \prime}}{ }_{b}\left(G_{x y^{\prime} ; a p^{\prime \prime \prime}} G_{x^{\prime} y ; q^{\prime \prime} d^{\prime}}+G_{x y ; a d^{\prime}} G_{x^{\prime} y^{\prime} ; q^{\prime \prime} p^{\prime \prime \prime}}\right. \\
&\left.+G_{y x^{\prime} ; q^{\prime \prime} d^{\prime}} G_{y^{\prime} x ; a p^{\prime \prime \prime}}+G_{y x ; a d^{\prime}} G_{y^{\prime} x^{\prime} ; q^{\prime \prime} p^{\prime \prime \prime}}\right) \\
&+g_{d^{\prime}} p^{\prime \prime \prime \prime} g^{q^{\prime \prime}}{ }_{b}\left(G_{x y^{\prime} ; a p^{\prime \prime \prime}} G_{x^{\prime} y ; q^{\prime \prime} c^{\prime}}+G_{x y ; a c^{\prime}} G_{x^{\prime} y^{\prime} ; q^{\prime \prime} p^{\prime \prime \prime}}\right. \\
&\left.\left.+G_{y x^{\prime} ; q^{\prime \prime} c^{\prime}} G_{y^{\prime} x ; a p^{\prime \prime \prime}}+G_{y x ; a c^{\prime}} G_{y^{\prime} x^{\prime} ; q^{\prime \prime} p^{\prime \prime \prime}}\right)\right] .
\end{aligned}
$$

If we now let $x^{\prime} \rightarrow x$ and $y^{\prime} \rightarrow y$, the contribution to the noise kernel is (including the factor of $\frac{1}{8}$ present in the definition of the noise kernel):

$$
\frac{1}{8}\left[(1-2 \xi)^{2}\left(G_{x y ; a d^{\prime}} G_{x y ; b c^{\prime}}+G_{x y ; a c^{\prime}} G_{x y ; b d^{\prime}}\right)+(1-2 \xi)^{2}\left(G_{y x ; a d^{\prime}} G_{y x ; b c^{\prime}}+G_{y x ; a c^{\prime}} G_{y x ; b d^{\prime}}\right)\right]
$$

That this term can be written as the sum of a part involving $G_{x y}$ and one involving $G_{y x}$ is a general property of the entire noise kernel. It thus takes the form

$$
N_{a b c^{\prime} d^{\prime}}(x, y)=N_{a b c^{\prime} d^{\prime}}\left[G_{+}(x, y)\right]+N_{a b c^{\prime} d^{\prime}}\left[G_{+}(y, x)\right]
$$

We will present the form of the functional $N_{a b c^{\prime} d^{\prime}}[G]$ shortly. First we note, that for $x$ and $y$ time-like separated, the above split of the noise kernel allows us to express it in terms of the Feynman (time ordered) Green's function $G_{\mathrm{F}}(x, y)$ and the Dyson (anti-time ordered) Green's function $G_{\mathrm{D}}(x, y)$ :

$$
N_{a b c^{\prime} d^{\prime}}(x, y)=N_{a b c^{\prime} d^{\prime}}\left[G_{\mathrm{F}}(x, y)\right]+N_{a b c^{\prime} d^{\prime}}\left[G_{\mathrm{D}}(x, y)\right]
$$

This can be connected with the zeta-function approach to this problem [302] as follows: Recall that when the quantum stress-tensor fluctuations determined in the Euclidean section is analytically continued back to Lorentzian signature $(\tau \rightarrow i t)$, the time-ordered product results. On the other hand, if the continuation is $\tau \rightarrow-i t$, the anti-time ordered product results. With this in mind, the noise kernel is seen to be related to the quantum stress-tensor fluctuations derived via the effective action as

$$
16 N_{a b c^{\prime} d^{\prime}}=\left.\Delta T_{a b c^{\prime} d^{\prime}}^{2}\right|_{t=-i \tau, t^{\prime}=-i \tau^{\prime}}+\left.\Delta T_{a b c^{\prime} d^{\prime}}^{2}\right|_{t=i \tau, t^{\prime}=i \tau^{\prime}}
$$

The complete form of the functional $N_{a b c^{\prime} d^{\prime}}[G]$ is

$$
N_{a b c^{\prime} d^{\prime}}[G]=\tilde{N}_{a b c^{\prime} d^{\prime}}[G]+g_{a b} \tilde{N}_{c^{\prime} d^{\prime}}[G]+g_{c^{\prime} d^{\prime}} \tilde{N}_{a b}^{\prime}[G]+g_{a b} g_{c^{\prime} d^{\prime}} \tilde{N}[G]
$$


with

$$
\begin{aligned}
& 8 \tilde{N}_{a b c^{\prime} d^{\prime}}[G]=(1-2 \xi)^{2}\left(G_{; c^{\prime} b} G_{; d^{\prime} a}+G_{; c^{\prime} a} G_{; d^{\prime} b}\right)+4 \xi^{2}\left(G_{; c^{\prime} d^{\prime}} G_{; a b}+G G_{; a b c^{\prime} d^{\prime}}\right) \\
& -2 \xi(1-2 \xi)\left(G_{; b} G_{; c^{\prime} a d^{\prime}}+G_{; a} G_{; c^{\prime} b d^{\prime}}+G_{; d^{\prime}} G_{; a b c^{\prime}}+G_{; c^{\prime}} G_{; a b d^{\prime}}\right) \\
& +2 \xi(1-2 \xi)\left(G_{; a} G_{; b} R_{c^{\prime} d^{\prime}}+G_{; c^{\prime}} G_{; d^{\prime}} R_{a b}\right) \\
& -4 \xi^{2}\left(G_{; a b} R_{c^{\prime} d^{\prime}}+G_{; c^{\prime} d^{\prime}} R_{a b}\right) G+2 \xi^{2} R_{c^{\prime} d^{\prime}} R_{a b} G^{2}, \\
& 8 \tilde{N}_{a b}^{\prime}[G]=2(1-2 \xi)\left[\left(2 \xi-\frac{1}{2}\right) G_{; p^{\prime} b} G_{;}^{p^{\prime}}{ }_{a}+\xi\left(G_{; b} G_{; p^{\prime} a}{ }^{p^{\prime}}+G_{; a} G_{; p^{\prime} b} p^{p^{\prime}}\right)\right] \\
& -4 \xi\left[\left(2 \xi-\frac{1}{2}\right) G_{;}^{p^{\prime}} G_{; a b p^{\prime}}+\xi\left(G_{; p^{\prime}}{ }^{p^{\prime}} G_{; a b}+G G_{; a b p^{\prime}}{ }^{p^{\prime}}\right)\right] \\
& -\left(m^{2}+\xi R^{\prime}\right)\left[(1-2 \xi) G_{; a} G_{; b}-2 G \xi G_{; a b}\right] \\
& +2 \xi\left[\left(2 \xi-\frac{1}{2}\right) G_{; p^{\prime}} G_{;}{ }^{p^{\prime}}+2 G \xi G_{; p^{\prime}}{ }^{p^{\prime}}\right] R_{a b}-\left(m^{2}+\xi R^{\prime}\right) \xi R_{a b} G^{2}, \\
& 8 \tilde{N}[G]=2\left(2 \xi-\frac{1}{2}\right)^{2} G_{; p^{\prime} q} G_{;}^{p^{\prime} q}+4 \xi^{2}\left(G_{; p^{\prime}} p^{\prime} G_{; q}{ }^{q}+G G_{; p}{ }^{p} q^{q^{\prime}}\right) \\
& +4 \xi\left(2 \xi-\frac{1}{2}\right)\left(G_{; p} G_{; q^{\prime}} p q^{\prime}+G_{;}^{p^{\prime}} G_{; q} q^{p^{\prime}}\right) \\
& \text { - }\left(2 \xi-\frac{1}{2}\right)\left[\left(m^{2}+\xi R\right) G_{; p^{\prime}} G_{;}^{p^{\prime}}+\left(m^{2}+\xi R^{\prime}\right) G_{; p} G^{; p}\right] \\
& -2 \xi\left[\left(m^{2}+\xi R\right) G_{; p^{\prime}} p^{\prime}+\left(m^{2}+\xi R^{\prime}\right) G_{; p}^{p}\right] G \\
& +\frac{1}{2}\left(m^{2}+\xi R\right)\left(m^{2}+\xi R^{\prime}\right) G^{2} \text {. }
\end{aligned}
$$

\subsubsection{Trace of the noise kernel}

One of the most interesting and surprising results to come out of the investigations of the quantum stress tensor undertaken in the 1970s was the discovery of the trace anomaly [77, 102]. When the trace of the stress tensor $T=g^{a b} T_{a b}$ is evaluated for a field configuration that satisfies the field equation (2), the trace is seen to vanish for massless conformally-coupled fields. When this analysis is carried over to the renormalized expectation value of the quantum stress tensor, the trace no longer vanishes. Wald [360] showed that this was due to the failure of the renormalized Hadamard function $G_{\text {ren }}\left(x, x^{\prime}\right)$ to be symmetric in $x$ and $x^{\prime}$, implying that it does not necessarily satisfy the field equation (2) in the variable $x^{\prime}$. (The definition of $G_{\text {ren }}\left(x, x^{\prime}\right)$ in the context of point separation will come next.)

With this in mind, we can now determine the noise associated with the trace. Taking the trace 
at both points $x$ and $y$ of the noise kernel functional (74) yields

$$
\begin{aligned}
N[G]= & g^{a b} g^{c^{\prime} d^{\prime}} N_{a b c^{\prime} d^{\prime}}[G] \\
= & -3 G \xi\left[\left(m^{2}+\frac{1}{2} \xi R\right) G_{; p^{\prime}} p^{\prime}+\left(m^{2}+\frac{1}{2} \xi R^{\prime}\right) G_{; p} p\right] \\
& +\frac{9 \xi^{2}}{2}\left(G_{; p^{\prime}}{ }^{p^{\prime}} G_{; p}{ }^{p}+G G_{; p}{ }^{p^{\prime}} p^{\prime}\right)+\left(m^{2}+\frac{1}{2} \xi R\right)\left(m^{2}+\frac{1}{2} \xi R^{\prime}\right) G^{2} \\
& +3\left(\frac{1}{6}-\xi\right)\left[3\left(\frac{1}{6}-\xi\right) G_{; p^{\prime} p} G_{;}^{p^{\prime} p}-3 \xi\left(G_{; p} G_{; p^{\prime}} p^{\prime}+G_{; p^{\prime}} G_{; p} p p^{\prime}\right)\right. \\
& \left.+\left(m^{2}+\frac{1}{2} \xi R\right) G_{; p^{\prime}} G_{;}^{p^{\prime}}+\left(m^{2}+\frac{1}{2} \xi R^{\prime}\right) G_{; p} G^{; p}\right]
\end{aligned}
$$

For the massless conformal case, this reduces to

$$
N[G]=\frac{1}{144}\left\{R R^{\prime} G^{2}-6 G\left(R \square^{\prime}+R^{\prime} \square\right) G+18\left[(\square G)\left(\square^{\prime} G\right)+\square^{\prime} \square G\right]\right\},
$$

which holds for any function $G(x, y)$. For $G$ as the Green's function, it satisfies the field equation (2):

$$
\square G=\left(m^{2}+\xi R\right) G .
$$

We will assume only that the Green's function satisfies the field equation in its first variable. Using the fact $\square^{\prime} R=0$ (because the covariant derivatives act at a different point than at which $R$ is supported), it follows that

$$
\square^{\prime} \square G=\left(m^{2}+\xi R\right) \square^{\prime} G .
$$

With these results, the noise kernel trace becomes

$$
\begin{aligned}
N[G]= & \frac{1}{2}\left[m^{2}(1-3 \xi)+3 R\left(\frac{1}{6}-\xi\right) \xi\right]\left[G^{2}\left(2 m^{2}+R^{\prime} \xi\right)+(1-6 \xi) G_{; p^{\prime}} G_{;}^{p^{\prime}}-6 G \xi G_{; p^{\prime}} p^{\prime}\right] \\
& +\frac{1}{2}\left(\frac{1}{6}-\xi\right)\left[3\left(2 m^{2}+R^{\prime} \xi\right) G_{; p} G^{; p}-18 \xi G_{; p} G_{; p^{\prime}} p p^{\prime}+18\left(\frac{1}{6}-\xi\right) G_{; p^{\prime} p} G_{;}^{p^{\prime} p}\right],
\end{aligned}
$$

which vanishes for the massless conformal case. We have thus shown, based solely on the definition of the point-separated noise kernel, that there is no noise associated with the trace anomaly. This result obtained in [305] is completely general since it is assumed that the Green's function is only satisfying the field equations in its first variable; an alternative proof of this result was given in [258]. This condition holds not just for the classical field case, but also for the regularized quantum case, where one does not expect the Green's function to satisfy the field equation in both variables. One can see this result from the simple observation used in Section 3; since the trace anomaly is known to be locally determined and quantum-state independent, whereas the noise present in the quantum field is non-local, it is hard to find a noise associated with it. This general result is in agreement with previous findings [58, 73, 206] derived from the Feynman-Vernon influence-functional formalism [107, 108] for some particular cases. 


\section{Metric Fluctuations in Minkowski Spacetime}

Although the Minkowski vacuum is an eigenstate of the total four-momentum operator of a field in Minkowski spacetime, it is not an eigenstate of the stress-energy operator. Hence, even for those solutions of semiclassical gravity, such as the Minkowski metric, for which the expectation value of the stress-energy operator can always be chosen to be zero, the fluctuations of this operator are non-vanishing. This fact leads us to consider the stochastic metric perturbations induced by these fluctuations.

Here we derive the Einstein-Langevin equation for the metric perturbations in a Minkowski background. We solve this equation for the linearized Einstein tensor and compute the associated two-point correlation functions, as well as the two-point correlation functions for the metric perturbations. Even though in this case we expect to have negligibly small values for these correlation functions for points separated by lengths larger than the Planck length, there are several reasons why it is worth carrying out this calculation.

On the one hand, these are the first backreaction solutions of the full Einstein-Langevin equation. There are analogous solutions to a "reduced" version of this equation inspired in a "minisuperspace" model [52, 74], and there is also a previous attempt to obtain a solution to the Einstein-Langevin equation in [73], but there the non-local terms in the Einstein-Langevin equation are neglected.

On the other hand, the results of this calculation, which confirm our expectations that gravitational fluctuations are negligible at length scales larger than the Planck length, but also predict that the fluctuations are strongly suppressed on small scales, can be considered a first test of stochastic semiclassical gravity. These results also reveal an important connection between stochastic gravity and the large $N$ expansion of quantum gravity. In addition, they are used in Section 6.5 to study the stability of the Minkowski metric as a solution of semiclassical gravity, which constitutes an application of the validity criterion introduced in Section 3.3. This calculation also requires a discussion of the problems posed by the so-called runaway solutions, which arise in the backreaction equations of semiclassical and stochastic gravity, and some of the methods to deal with them. As a result we conclude that Minkowski spacetime is a stable and valid solution of semiclassical gravity.

We advise the reader that Section 6 is rather technical since it deals with an explicit non trivial backreaction computation in stochastic gravity. We tried to make it reasonable self-contained and detailed, however a more detailed exposition can be found in [259].

\subsection{Perturbations around Minkowski spacetime}

The Minkowski metric $\eta_{a b}$ in a manifold $\mathcal{M}$, which is topologically $\mathbb{R}^{4}$, together with the usual Minkowski vacuum, denoted as $|0\rangle$, is the simplest solution to the semiclassical Einstein equation (8), the so-called trivial solution of semiclassical gravity [110]. It constitutes the ground state of semiclassical gravity. In fact, we can always choose a renormalization scheme in which the renormalized expectation value $\left\langle 0\left|\hat{T}_{\mathrm{R}}^{a b}[\eta]\right| 0\right\rangle=0$. Thus, the Minkowski spacetime $\left(\mathbb{R}^{4}, \eta_{a b}\right)$ plus the vacuum state $|0\rangle$ is a solution to the semiclassical Einstein equation with renormalized cosmological constant $\Lambda=0$. The fact that the vacuum expectation value of the renormalized stress-energy operator in Minkowski spacetime should vanish was originally proposed by Wald [359], and it may be understood as a renormalization convention [121, 135]. Note that other possible solutions of semiclassical gravity with zero vacuum expectation value of the stress-energy tensor are the exact gravitational plane waves, since they are known to be vacuum solutions of Einstein equations, which induce neither particle creation nor vacuum polarization [91, 125, 128].

As we have already mentioned, the vacuum $|0\rangle$ is an eigenstate of the total four-momentum operator in Minkowski spacetime, but not an eigenstate of $\hat{T}_{a b}^{R}[\eta]$. Hence, even in the Minkowski background there are quantum fluctuations in the stress-energy tensor and, as a result, the noise 
kernel does not vanish. This fact leads us to consider stochastic corrections to this class of trivial solutions of semiclassical gravity. Since in this case the Wightman and Feynman functions (44), their values in the two-point coincidence limit and the products of derivatives of two of such functions appearing in expressions (45) and (46) are known in dimensional regularization, we can compute the Einstein-Langevin equation using the methods outlined in Sections 3 and 4.

To perform explicit calculations it is convenient to work in a global inertial coordinate system $\left\{x^{\mu}\right\}$ and in the associated basis, in which the components of the flat metric are simply $\eta_{\mu \nu}=$ $\operatorname{diag}(-1,1, \ldots, 1)$. In Minkowski spacetime, the components of the classical stress-energy tensor (4) reduce to

$$
T^{\mu \nu}[\eta, \phi]=\partial^{\mu} \phi \partial^{\nu} \phi-\frac{1}{2} \eta^{\mu \nu} \partial^{\rho} \phi \partial_{\rho} \phi-\frac{1}{2} \eta^{\mu \nu} m^{2} \phi^{2}+\xi\left(\eta^{\mu \nu} \square-\partial^{\mu} \partial^{\nu}\right) \phi^{2},
$$

where $\square \equiv \partial_{\mu} \partial^{\mu}$ and the formal expression for the components of the corresponding "operator" in dimensional regularization, see Equation (5), is

$$
\hat{T}_{n}^{\mu \nu}[\eta]=\frac{1}{2}\left\{\partial^{\mu} \hat{\phi}_{n}, \partial^{\nu} \hat{\phi}_{n}\right\}+\mathcal{D}^{\mu \nu} \hat{\phi}_{n}^{2}
$$

where $\mathcal{D}^{\mu \nu}$ is the differential operator (6), with $g_{\mu \nu}=\eta_{\mu \nu}, R_{\mu \nu}=0$, and $\nabla_{\mu}=\partial_{\mu}$. The field $\hat{\phi}_{n}(x)$ is the field operator in the Heisenberg representation in an $n$-dimensional Minkowski spacetime, which satisfies the Klein-Gordon equation (2). We use here a stress-energy tensor, which differs from the canonical one that corresponds to $\xi=0$; both tensors, however, define the same total momentum.

The Wightman and Feynman functions (44) for $g_{\mu \nu}=\eta_{\mu \nu}$ are well known:

$$
G_{n}^{+}(x, y)=i \Delta_{n}^{+}(x-y), \quad G_{F_{n}}(x, y)=\Delta_{F_{n}}(x-y)
$$

with

$$
\begin{aligned}
\Delta_{n}^{+}(x) & =-2 \pi i \int \frac{d^{n} k}{(2 \pi)^{n}} e^{i k x} \delta\left(k^{2}+m^{2}\right) \theta\left(k^{0}\right), \\
\Delta_{F_{n}}(x) & =-\int \frac{d^{n} k}{(2 \pi)^{n}} \frac{e^{i k x}}{k^{2}+m^{2}-i \epsilon} \quad \text { for } \epsilon \rightarrow 0^{+},
\end{aligned}
$$

where $k^{2} \equiv \eta_{\mu \nu} k^{\mu} k^{\nu}$ and $k x \equiv \eta_{\mu \nu} k^{\mu} x^{\nu}$. Note that the derivatives of these functions satisfy $\partial_{\mu}^{x} \Delta_{n}^{+}(x-y)=\partial_{\mu} \Delta_{n}^{+}(x-y)$ and $\partial_{\mu}^{y} \Delta_{n}^{+}(x-y)=-\partial_{\mu} \Delta_{n}^{+}(x-y)$, and similarly for the Feynman propagator $\Delta_{F_{n}}(x-y)$.

To write down the semiclassical Einstein equation (8) in $n$ dimensions for this case, we need to compute the vacuum expectation value of the stress-energy operator components (86). Since, from (87), we have that $\left\langle 0\left|\hat{\phi}_{n}^{2}(x)\right| 0\right\rangle=i \Delta_{F_{n}}(0)=i \Delta_{n}^{+}(0)$, which is a constant (independent of $x$ ), we have simply

$$
\left\langle 0\left|\hat{T}_{n}^{\mu \nu}[\eta]\right| 0\right\rangle=-i \int \frac{d^{n} k}{(2 \pi)^{n}} \frac{k^{\mu} k^{\nu}}{k^{2}+m^{2}-i \epsilon}=\frac{\eta^{\mu \nu}}{2}\left(\frac{m^{2}}{4 \pi}\right)^{n / 2} \Gamma\left(-\frac{n}{2}\right),
$$

where the integrals in dimensional regularization have been computed in the standard way [259], and where $\Gamma(z)$ is Euler's gamma function. The semiclassical Einstein equation (8) in $n$ dimensions before renormalization reduces now to

$$
\frac{\Lambda_{\mathrm{B}}}{8 \pi G_{\mathrm{B}}} \eta^{\mu \nu}=\mu^{-(n-4)}\left\langle 0\left|\hat{T}_{n}^{\mu \nu}[\eta]\right| 0\right\rangle .
$$

Thus, this equation simply sets the value of the bare coupling constant $\Lambda_{\mathrm{B}} / G_{\mathrm{B}}$. Note from Equation (89) that in order to have $\left\langle 0\left|\hat{T}_{\mathrm{R}}^{\mu \nu}\right| 0\right\rangle[\eta]=0$, the renormalized and regularized stress-energy 
tensor "operator" for a scalar field in Minkowski spacetime, see Equation (7), has to be defined as

$$
\hat{T}_{\mathrm{R}}^{\mu \nu}[\eta]=\mu^{-(n-4)} \hat{T}_{n}^{\mu \nu}[\eta]-\frac{\eta^{\mu \nu}}{2} \frac{m^{4}}{(4 \pi)^{2}}\left(\frac{m^{2}}{4 \pi \mu^{2}}\right)^{\frac{n-4}{2}} \Gamma\left(-\frac{n}{2}\right),
$$

which corresponds to a renormalization of the cosmological constant

$$
\frac{\Lambda_{\mathrm{B}}}{G_{\mathrm{B}}}=\frac{\Lambda}{G}-\frac{2}{\pi} \frac{m^{4}}{n(n-2)} \kappa_{n}+\mathcal{O}(n-4),
$$

where

$$
\kappa_{n} \equiv \frac{1}{n-4}\left(\frac{e^{\gamma} m^{2}}{4 \pi \mu^{2}}\right)^{\frac{n-4}{2}}=\frac{1}{n-4}+\frac{1}{2} \ln \left(\frac{e^{\gamma} m^{2}}{4 \pi \mu^{2}}\right)+\mathcal{O}(n-4),
$$

with $\gamma$ being Euler's constant. In the case of a massless scalar field, $m^{2}=0$, one simply has $\Lambda_{\mathrm{B}} / G_{\mathrm{B}}=\Lambda / G$. Introducing this renormalized coupling constant into Equation (90), we can take the limit $n \rightarrow 4$. We find that, for $\left(\mathbb{R}^{4}, \eta_{a b},|0\rangle\right)$ to satisfy the semiclassical Einstein equation, we must take $\Lambda=0$.

We can now write down the Einstein-Langevin equations for the components $h_{\mu \nu}$ of the stochastic metric perturbation in dimensional regularization. In our case, using $\left\langle 0\left|\hat{\phi}_{n}^{2}(x)\right| 0\right\rangle=i \Delta_{F_{n}}(0)$ and the explicit expression of Equation (41), we obtain

$$
\begin{aligned}
\frac{1}{8 \pi G_{\mathrm{B}}}[ & \left.G^{(1) \mu \nu}+\Lambda_{\mathrm{B}}\left(h^{\mu \nu}-\frac{1}{2} \eta^{\mu \nu} h\right)\right](x)-\frac{4}{3} \alpha_{\mathrm{B}} D^{(1) \mu \nu}(x)-2 \beta_{\mathrm{B}} B^{(1) \mu \nu}(x) \\
& \quad-\xi G^{(1) \mu \nu}(x) \mu^{-(n-4)} i \Delta_{F_{n}}(0)+\frac{1}{2} \int d^{n} y \mu^{-(n-4)} H_{n}^{\mu \nu \alpha \beta}(x, y) h_{\alpha \beta}(y)=\xi^{\mu \nu}(x) .
\end{aligned}
$$

The indices in $h_{\mu \nu}$ are raised with the Minkowski metric and $h \equiv h_{\rho}^{\rho}$; here a superindex ${ }^{(1)}$ denotes the components of a tensor linearized around the flat metric. Note that in $n$ dimensions the two-point correlation functions for the field $\xi^{\mu \nu}$ is written as

$$
\left\langle\xi^{\mu \nu}(x) \xi^{\alpha \beta}(y)\right\rangle_{\mathrm{s}}=\mu^{-2(n-4)} N_{n}^{\mu \nu \alpha \beta}(x, y) .
$$

Explicit expressions for $D^{(1) \mu \nu}$ and $B^{(1) \mu \nu}$ are given by

$$
D^{(1) \mu \nu}(x)=\frac{1}{2} \mathcal{F}_{x}^{\mu \nu \alpha \beta} h_{\alpha \beta}(x), \quad B^{(1) \mu \nu}(x)=2 \mathcal{F}_{x}^{\mu \nu} \mathcal{F}_{x}^{\alpha \beta} h_{\alpha \beta}(x),
$$

with the differential operators $\mathcal{F}_{x}^{\mu \nu} \equiv \eta^{\mu \nu} \square_{x}-\partial_{x}^{\mu} \partial_{x}^{\nu}$ and $\mathcal{F}_{x}^{\mu \nu \alpha \beta} \equiv 3 \mathcal{F}_{x}^{\mu(\alpha} \mathcal{F}_{x}^{\beta) \nu}-\mathcal{F}_{x}^{\mu \nu} \mathcal{F}_{x}^{\alpha \beta}$.

\subsection{The kernels in the Minkowski background}

Since the two kernels (43) are free of ultraviolet divergences in the limit $n \rightarrow 4$, we can deal directly with the $F^{\mu \nu \alpha \beta}(x-y) \equiv \lim _{n \rightarrow 4} \mu^{-2(n-4)} F_{n}^{\mu \nu \alpha \beta}$ in Equation (42). The kernels $N^{\mu \nu \alpha \beta}(x, y)=$ $\operatorname{Re} F^{\mu \nu \alpha \beta}(x-y)$ and $H_{\mathrm{A}}^{\mu \nu \alpha \beta}(x, y)=\operatorname{Im} F^{\mu \nu \alpha \beta}(x-y)$ are actually the components of the "physical" noise and dissipation kernels that will appear in the Einstein-Langevin equations once the renormalization procedure has been carried out. The bitensor $F^{\mu \nu \alpha \beta}$ can be expressed in terms of the Wightman function in four spacetime dimensions, according to Equation (45). The different terms in this kernel can be easily computed using the integrals

$$
I(p) \equiv \int \frac{d^{4} k}{(2 \pi)^{4}} \delta\left(k^{2}+m^{2}\right) \theta\left(-k^{0}\right) \delta\left[(k-p)^{2}+m^{2}\right] \theta\left(k^{0}-p^{0}\right)
$$


and $I^{\mu_{1} \ldots \mu_{r}}(p)$, which are defined as in Equation (97) by inserting the momenta $k^{\mu_{1}} \ldots k^{\mu_{r}}$ with $r=1, \ldots, 4$ into the integrand. All these integrals can be expressed in terms of $I(p)$; see [259] for the explicit expressions. It is convenient to separate $I(p)$ into its even and odd parts with respect to the variables $p^{\mu}$ as

$$
I(p)=I_{\mathrm{S}}(p)+I_{\mathrm{A}}(p),
$$

where $I_{\mathrm{S}}(-p)=I_{\mathrm{S}}(p)$ and $I_{\mathrm{A}}(-p)=-I_{\mathrm{A}}(p)$. These two functions are explicitly given by

$$
\begin{aligned}
& I_{\mathrm{S}}(p)=\frac{1}{8(2 \pi)^{3}} \theta\left(-p^{2}-4 m^{2}\right) \sqrt{1+4 \frac{m^{2}}{p^{2}}} \\
& I_{\mathrm{A}}(p)=\frac{-1}{8(2 \pi)^{3}} \operatorname{sign}\left(p^{0}\right) \theta\left(-p^{2}-4 m^{2}\right) \sqrt{1+4 \frac{m^{2}}{p^{2}}} .
\end{aligned}
$$

After some manipulations, we find

$$
\begin{aligned}
F^{\mu \nu \alpha \beta}(x)= & \frac{\pi^{2}}{45} \mathcal{F}_{x}^{\mu \nu \alpha \beta} \int \frac{d^{4} p}{(2 \pi)^{4}} e^{-i p x}\left(1+4 \frac{m^{2}}{p^{2}}\right)^{2} I(p) \\
& +\frac{8 \pi^{2}}{9} \mathcal{F}_{x}^{\mu \nu} \mathcal{F}_{x}^{\alpha \beta} \int \frac{d^{4} p}{(2 \pi)^{4}} e^{-i p x}\left(3 \Delta \xi+\frac{m^{2}}{p^{2}}\right)^{2} I(p),
\end{aligned}
$$

where $\Delta \xi \equiv \xi-\frac{1}{6}$. The real and imaginary parts of the last expression, which yield the noise and dissipation kernels, are easily recognized as the terms containing $I_{\mathrm{S}}(p)$ and $I_{\mathrm{A}}(p)$, respectively. To write them explicitly, it is useful to introduce the new kernels

$$
\begin{aligned}
N_{\mathrm{A}}\left(x ; m^{2}\right) & \equiv \frac{1}{480 \pi} \int \frac{d^{4} p}{(2 \pi)^{4}} e^{i p x} \theta\left(-p^{2}-4 m^{2}\right) \sqrt{1+4 \frac{m^{2}}{p^{2}}}\left(1+4 \frac{m^{2}}{p^{2}}\right)^{2}, \\
N_{\mathrm{B}}\left(x ; m^{2}, \Delta \xi\right) & \equiv \frac{1}{72 \pi} \int \frac{d^{4} p}{(2 \pi)^{4}} e^{i p x} \theta\left(-p^{2}-4 m^{2}\right) \sqrt{1+4 \frac{m^{2}}{p^{2}}}\left(3 \Delta \xi+\frac{m^{2}}{p^{2}}\right)^{2}, \\
D_{\mathrm{A}}\left(x ; m^{2}\right) & \equiv \frac{-i}{480 \pi} \int \frac{d^{4} p}{(2 \pi)^{4}} e^{i p x} \operatorname{sign}\left(p^{0}\right) \theta\left(-p^{2}-4 m^{2}\right) \sqrt{1+4 \frac{m^{2}}{p^{2}}}\left(1+4 \frac{m^{2}}{p^{2}}\right)^{2}, \\
D_{\mathrm{B}}\left(x ; m^{2}, \Delta \xi\right) & \equiv \frac{-i}{72 \pi} \int \frac{d^{4} p}{(2 \pi)^{4}} e^{i p x} \operatorname{sign}\left(p^{0}\right) \theta\left(-p^{2}-4 m^{2}\right) \sqrt{1+4 \frac{m^{2}}{p^{2}}}\left(3 \Delta \xi+\frac{m^{2}}{p^{2}}\right)^{2} .
\end{aligned}
$$

Finally, we get

$$
\begin{aligned}
& N^{\mu \nu \alpha \beta}(x, y)=\frac{1}{6} \mathcal{F}_{x}^{\mu \nu \alpha \beta} N_{\mathrm{A}}\left(x-y ; m^{2}\right)+\mathcal{F}_{x}^{\mu \nu} \mathcal{F}_{x}^{\alpha \beta} N_{\mathrm{B}}\left(x-y ; m^{2}, \Delta \xi\right) \\
& H_{\mathrm{A}}^{\mu \nu \alpha \beta}(x, y)=\frac{1}{6} \mathcal{F}_{x}^{\mu \nu \alpha \beta} D_{\mathrm{A}}\left(x-y ; m^{2}\right)+\mathcal{F}_{x}^{\mu \nu} \mathcal{F}_{x}^{\alpha \beta} D_{\mathrm{B}}\left(x-y ; m^{2}, \Delta \xi\right)
\end{aligned}
$$

Notice that the noise and dissipation kernels defined in Equation (101) are actually real because, for the noise kernels, only the $\cos p x$ terms of the exponentials $e^{i p x}$ contribute to the integrals, and, for the dissipation kernels, the only contribution of such exponentials comes from the $i \sin p x$ terms.

The evaluation of the kernel $H_{\mathrm{S}_{n}}^{\mu \nu \alpha \beta}(x, y)$ is a more involved task. Since this kernel contains divergences in the limit $n \rightarrow 4$, we use dimensional regularization. Using Equation (46), this kernel can be written in terms of the Feynman propagator (88) as

$$
\mu^{-(n-4)} H_{\mathrm{S}_{n}}^{\mu \nu \alpha \beta}(x, y)=\operatorname{Im} K^{\mu \nu \alpha \beta}(x-y)
$$


where

$$
\begin{aligned}
K^{\mu \nu \alpha \beta}(x) \equiv-\mu^{-(n-4)}\{ & 2 \partial^{\mu} \partial^{(\alpha} \Delta_{F_{n}}(x) \partial^{\beta)} \partial^{\nu} \Delta_{F_{n}}(x)+2 \mathcal{D}^{\mu \nu}\left(\partial^{\alpha} \Delta_{F_{n}}(x) \partial^{\beta} \Delta_{F_{n}}(x)\right) \\
+ & 2 \mathcal{D}^{\alpha \beta}\left(\partial^{\mu} \Delta_{F_{n}}(x) \partial^{\nu} \Delta_{F_{n}}(x)\right)+2 \mathcal{D}^{\mu \nu} \mathcal{D}^{\alpha \beta}\left(\Delta_{F_{n}}^{2}(x)\right) \\
+ & {\left[\eta^{\mu \nu} \partial^{(\alpha} \Delta_{F_{n}}(x) \partial^{\beta)}+\eta^{\alpha \beta} \partial^{(\mu} \Delta_{F_{n}}(x) \partial^{\nu)}+\Delta_{F_{n}}(0)\left(\eta^{\mu \nu} \mathcal{D}^{\alpha \beta}+\eta^{\alpha \beta} \mathcal{D}^{\mu \nu}\right)\right.} \\
& \left.\left.+\frac{1}{4} \eta^{\mu \nu} \eta^{\alpha \beta}\left(\Delta_{F_{n}}(x) \square-m^{2} \Delta_{F_{n}}(0)\right)\right] \delta^{n}(x)\right\} .
\end{aligned}
$$

Let us define the integrals

$$
J_{n}(p) \equiv \mu^{-(n-4)} \int \frac{d^{n} k}{(2 \pi)^{n}} \frac{1}{\left(k^{2}+m^{2}-i \epsilon\right)\left[(k-p)^{2}+m^{2}-i \epsilon\right]},
$$

and $J_{n}^{\mu_{1} \ldots \mu_{r}}(p)$ obtained by inserting the momenta $k^{\mu_{1}} \ldots k^{\mu_{r}}$ into Equation (105), together with

$$
I_{0_{n}} \equiv \mu^{-(n-4)} \int \frac{d^{n} k}{(2 \pi)^{n}} \frac{1}{\left(k^{2}+m^{2}-i \epsilon\right)},
$$

and $I_{0_{n}}^{\mu_{1} \ldots \mu_{r}}$, which are also obtained by inserting momenta into the integrand. Then the different terms in Equation (104) can be computed; these integrals are explicitly given in [259]. It is found that $I_{0_{n}}^{\mu}=0$, and the remaining integrals can be written in terms of $I_{0_{n}}$ and $J_{n}(p)$. It is useful to introduce the projector $P^{\mu \nu}$ orthogonal to $p^{\mu}$ and the tensor $P^{\mu \nu \alpha \beta}$ as

$$
p^{2} P^{\mu \nu} \equiv \eta^{\mu \nu} p^{2}-p^{\mu} p^{\nu}, \quad P^{\mu \nu \alpha \beta} \equiv 3 P^{\mu(\alpha} P^{\beta) \nu}-P^{\mu \nu} P^{\alpha \beta} .
$$

Then the action of the operator $\mathcal{F}_{x}^{\mu \nu}$ is simply written as $\mathcal{F}_{x}^{\mu \nu} \int d^{n} p e^{i p x} f(p)=-\int d^{n} p e^{i p x} f(p) p^{2} P^{\mu \nu}$, where $f(p)$ is an arbitrary function of $p^{\mu}$.

Finally, after a rather long but straightforward calculation, and after expanding around $n=4$, we get

$$
\begin{aligned}
& K^{\mu \nu \alpha \beta}(x)=\frac{i}{(4 \pi)^{2}}\left\{\kappa _ { n } \left[\frac{1}{90} \mathcal{F}_{x}^{\mu \nu \alpha \beta} \delta^{n}(x)+4 \Delta \xi^{2} \mathcal{F}_{x}^{\mu \nu} \mathcal{F}_{x}^{\alpha \beta} \delta^{n}(x)\right.\right. \\
&+\frac{2}{3} \frac{m^{2}}{(n-2)}\left(\eta^{\mu \nu} \eta^{\alpha \beta} \square_{x}-\eta^{\mu(\alpha} \eta^{\beta) \nu} \square_{x}+\eta^{\mu(\alpha} \partial_{x}^{\beta)} \partial_{x}^{\nu}\right. \\
&\left.+\eta^{\nu(\alpha} \partial_{x}^{\beta)} \partial_{x}^{\mu}-\eta^{\mu \nu} \partial_{x}^{\alpha} \partial_{x}^{\beta}-\eta^{\alpha \beta} \partial_{x}^{\mu} \partial_{x}^{\nu}\right) \delta^{n}(x) \\
&\left.\quad+\frac{4 m^{4}}{n(n-2)}\left(2 \eta^{\mu(\alpha} \eta^{\beta) \nu}-\eta^{\mu \nu} \eta^{\alpha \beta}\right) \delta^{n}(x)\right] \\
&+\frac{1}{180} \mathcal{F}_{x}^{\mu \nu \alpha \beta} \int \frac{d^{n} p}{(2 \pi)^{n}} e^{i p x}\left(1+4 \frac{m^{2}}{p^{2}}\right)^{2} \bar{\phi}\left(p^{2}\right) \\
&+\frac{2}{9} \mathcal{F}_{x}^{\mu \nu} \mathcal{F}_{x}^{\alpha \beta} \int \frac{d^{n} p}{(2 \pi)^{n}} e^{i p x}\left(3 \Delta \xi+\frac{m^{2}}{p^{2}}\right)^{2} \bar{\phi}\left(p^{2}\right) \\
&-\left[\frac{4}{675} \mathcal{F}_{x}^{\mu \nu \alpha \beta}+\frac{1}{270}(60 \xi-11) \mathcal{F}_{x}^{\mu \nu} \mathcal{F}_{x}^{\alpha \beta}\right] \delta^{n}(x) \\
&-\left.m^{2}\left[\frac{2}{135} \mathcal{F}_{x}^{\mu \nu \alpha \beta}+\frac{1}{27} \mathcal{F}_{x}^{\mu \nu} \mathcal{F}_{x}^{\alpha \beta}\right] \Delta_{n}(x)\right\}+\mathcal{O}(n-4),
\end{aligned}
$$


where $\kappa_{n}$ has been defined in Equation (93), and $\bar{\phi}\left(p^{2}\right)$ and $\Delta_{n}(x)$ are given by

$$
\begin{aligned}
\bar{\phi}\left(p^{2}\right) & \equiv \int_{0}^{1} d \alpha \ln \left(1+\frac{p^{2}}{m^{2}} \alpha(1-\alpha)-i \epsilon\right)=-i \pi \theta\left(-p^{2}-4 m^{2}\right) \sqrt{1+4 \frac{m^{2}}{p^{2}}}+\varphi\left(p^{2}\right), \\
\Delta_{n}(x) & \equiv \int \frac{d^{n} p}{(2 \pi)^{n}} e^{i p x} \frac{1}{p^{2}}
\end{aligned}
$$

where

$$
\varphi\left(p^{2}\right) \equiv \int_{0}^{1} d \alpha \ln \left|1+\frac{p^{2}}{m^{2}} \alpha(1-\alpha)\right|
$$

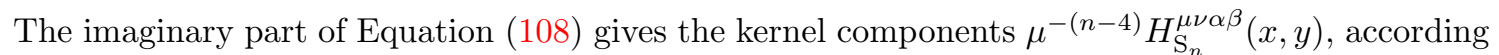
to Equation (103). It can be easily obtained by multiplying this expression by $-i$ and retaining only the real part $\varphi\left(p^{2}\right)$ of the function $\bar{\phi}\left(p^{2}\right)$.

\subsection{The Einstein-Langevin equation}

With the previous results for the kernels we can now write the $n$-dimensional Einstein-Langevin equation (94), previous to the renormalization. Also taking into account Equations (89) and (90), we may finally write:

$$
\begin{aligned}
& \frac{1}{8 \pi G_{\mathrm{B}}} G^{(1) \mu \nu}(x)-\frac{4}{3} \alpha_{\mathrm{B}} D^{(1) \mu \nu}(x)-2 \beta_{\mathrm{B}} B^{(1) \mu \nu}(x) \\
& +\frac{\kappa_{n}}{(4 \pi)^{2}}\left[-4 \Delta \xi \frac{m^{2}}{(n-2)} G^{(1) \mu \nu}+\frac{1}{90} D^{(1) \mu \nu} \Delta \xi^{2} B^{(1) \mu \nu}\right](x) \\
& +\frac{1}{2880 \pi^{2}}\left\{-\frac{16}{15} D^{(1) \mu \nu}(x)+\left(\frac{1}{6}-10 \Delta \xi\right) B^{(1) \mu \nu}(x)\right. \\
& +\int d^{n} y \int \frac{d^{n} p}{(2 \pi)^{n}} e^{i p(x-y)} \varphi\left(p^{2}\right)\left[\left(1+4 \frac{m^{2}}{p^{2}}\right)^{2} D^{(1) \mu \nu}(y)+10\left(3 \Delta \xi+\frac{m^{2}}{p^{2}}\right)^{2} B^{(1) \mu \nu}(y)\right] \\
& \left.\quad-\frac{m^{2}}{3} \int d^{n} y \Delta_{n}(x-y)\left(8 D^{(1) \mu \nu}+5 B^{(1) \mu \nu}\right)(y)\right\} \\
& +\frac{1}{2} \int d^{n} y \mu^{-(n-4)} H_{\mathrm{A}_{n}}^{\mu \nu \alpha \beta}(x, y) h_{\alpha \beta}(y)+\mathcal{O}(n-4)=\xi^{\mu \nu}(x) .
\end{aligned}
$$

Notice that the terms containing the bare cosmological constant have cancelled. These equations can now be renormalized; that is, we can now write the bare coupling constants as renormalized coupling constants plus some suitably-chosen counterterms, and take the limit as $n \rightarrow 4$. In order to carry out such a procedure, it is convenient to distinguish between massive and massless scalar fields. The details of the calculation can be found in [259]. 
It is convenient to introduce the two new kernels

$$
\begin{aligned}
H_{\mathrm{A}}\left(x ; m^{2}\right) \equiv & \frac{1}{480 \pi^{2}} \int \frac{d^{4} p}{(2 \pi)^{4}} e^{i p x} \\
& \times\left\{\left(1+4 \frac{m^{2}}{p^{2}}\right)^{2}\left[-i \pi \operatorname{sign}\left(p^{0}\right) \theta\left(-p^{2}-4 m^{2}\right) \sqrt{1+4 \frac{m^{2}}{p^{2}}}+\varphi\left(p^{2}\right)\right]-\frac{8}{3} \frac{m^{2}}{p^{2}}\right\}, \\
H_{\mathrm{B}}\left(x ; m^{2}, \Delta \xi\right) \equiv & \frac{1}{72 \pi^{2}} \int \frac{d^{4} p}{(2 \pi)^{4}} e^{i p x} \\
& \times\left\{\left(3 \Delta \xi+\frac{m^{2}}{p^{2}}\right)^{2}\left[-i \pi \operatorname{sign}\left(p^{0}\right) \theta\left(-p^{2}-4 m^{2}\right) \sqrt{1+4 \frac{m^{2}}{p^{2}}}+\varphi\left(p^{2}\right)\right]-\frac{1}{6} \frac{m^{2}}{p^{2}}\right\},
\end{aligned}
$$

where $\varphi\left(p^{2}\right)$ is given by the restriction to $n=4$ of expression (109). The renormalized coupling constants $1 / G, \alpha$ and $\beta$ are easily computed as in Equation (92). Substituting their expressions into Equation (111), we can take the limit as $n \rightarrow 4$. Using the fact that, for $n=4, D^{(1) \mu \nu}(x)=$ $\frac{3}{2} A^{(1) \mu \nu}(x)$, we obtain the corresponding semiclassical Einstein-Langevin equation.

For the massless case one needs the limit as $m \rightarrow 0$ of Equation (111). In this case it is convenient to separate $\kappa_{n}$ in Equation (93) as $\kappa_{n}=\tilde{\kappa}_{n}+\frac{1}{2} \ln \left(m^{2} / \mu^{2}\right)+\mathcal{O}(n-4)$, where

$$
\tilde{\kappa}_{n} \equiv \frac{1}{n-4}\left(\frac{e^{\gamma}}{4 \pi}\right)^{\frac{n-4}{2}}=\frac{1}{n-4}+\frac{1}{2} \ln \left(\frac{e^{\gamma}}{4 \pi}\right)+\mathcal{O}(n-4),
$$

and use that, from Equation (109), we have

$$
\lim _{m^{2} \rightarrow 0}\left[\varphi\left(p^{2}\right)+\ln \frac{m^{2}}{\mu^{2}}\right]=-2+\ln \left|\frac{p^{2}}{\mu^{2}}\right| .
$$

The coupling constants are then easily renormalized. We note that in the massless limit, the Newtonian gravitational constant is not renormalized and, in the conformal coupling case, $\Delta \xi=0$, we have that $\beta_{\mathrm{B}}=\beta$. Note also that, by making $m=0$ in Equation (101), the noise and dissipation kernels can be written as

$$
\begin{array}{ll}
N_{\mathrm{A}}\left(x ; m^{2}=0\right)=N(x), & N_{\mathrm{B}}\left(x ; m^{2}=0, \Delta \xi\right)=60 \Delta \xi^{2} N(x), \\
D_{\mathrm{A}}\left(x ; m^{2}=0\right)=D(x), & D_{\mathrm{B}}\left(x ; m^{2}=0, \Delta \xi\right)=60 \Delta \xi^{2} D(x),
\end{array}
$$

where

$$
N(x) \equiv \frac{1}{480 \pi} \int \frac{d^{4} p}{(2 \pi)^{4}} e^{i p x} \theta\left(-p^{2}\right), \quad D(x) \equiv \frac{-i}{480 \pi} \int \frac{d^{4} p}{(2 \pi)^{4}} e^{i p x} \operatorname{sign}\left(p^{0}\right) \theta\left(-p^{2}\right) .
$$

It is also convenient to introduce the new kernel

$$
\begin{aligned}
H\left(x ; \mu^{2}\right) & \equiv \frac{1}{480 \pi^{2}} \int \frac{d^{4} p}{(2 \pi)^{4}} e^{i p x}\left[\ln \left|\frac{p^{2}}{\mu^{2}}\right|-i \pi \operatorname{sign}\left(p^{0}\right) \theta\left(-p^{2}\right)\right] \\
& =\frac{1}{480 \pi^{2}} \lim _{\epsilon \rightarrow 0^{+}} \int \frac{d^{4} p}{(2 \pi)^{4}} e^{i p x} \ln \left(\frac{-\left(p^{0}+i \epsilon\right)^{2}+p^{i} p_{i}}{\mu^{2}}\right) .
\end{aligned}
$$

This kernel is real and can be written as the sum of an even part and an odd part in the variables $x^{\mu}$, where the odd part is the dissipation kernel $D(x)$. The Fourier transforms (116) and (117) can actually be computed and, thus, in this case, we have explicit expressions for the kernels in position space; see, for instance, [71, 169, 220]. 
Finally, the Einstein-Langevin equation for the physical stochastic perturbations $h_{\mu \nu}$ can be written in both cases, for $m \neq 0$ and for $m=0$, as

$$
\begin{gathered}
\frac{1}{8 \pi G} G^{(1) \mu \nu}(x)-2\left(\bar{\alpha} A^{(1) \mu \nu}(x)+\bar{\beta} B^{(1) \mu \nu}(x)\right) \\
+\frac{1}{4} \int d^{4} y\left[H_{\mathrm{A}}(x-y) A^{(1) \mu \nu}(y)+H_{\mathrm{B}}(x-y) B^{(1) \mu \nu}(y)\right]=\xi^{\mu \nu}(x),
\end{gathered}
$$

where, in terms of the renormalized constants $\alpha$ and $\beta$, the new constants are $\bar{\alpha}=\alpha+\left(3600 \pi^{2}\right)^{-1}$ and $\bar{\beta}=\beta-\left(\frac{1}{12}-5 \Delta \xi\right)\left(2880 \pi^{2}\right)^{-1}$. The kernels $H_{\mathrm{A}}(x)$ and $H_{\mathrm{B}}(x)$ are given by Equations $(112)$ when $m \neq 0$, and by $H_{\mathrm{A}}(x)=H\left(x ; \mu^{2}\right)$ and $H_{\mathrm{B}}(x)=60 \Delta \xi^{2} H\left(x ; \mu^{2}\right)$ when $m=0$. In the massless case, we can use the arbitrariness of the mass scale $\mu$ to eliminate one of the parameters $\bar{\alpha}$ or $\bar{\beta}$. The components of the Gaussian stochastic source $\xi^{\mu \nu}$ have zero mean value, and their two-point correlation functions are given by $\left\langle\xi^{\mu \nu}(x) \xi^{\alpha \beta}(y)\right\rangle_{\mathrm{s}}=N^{\mu \nu \alpha \beta}(x, y)$, where the noise kernel is given in Equation (102), which in the massless case reduces to Equation (115).

It is interesting to consider the massless conformally-coupled scalar field, i.e., the case $\Delta \xi=$ 0 , which is of particular interest because of its similarities with the electromagnetic field, and also because of its interest to cosmology; massive fields become conformally invariant when their masses are negligible compared to the spacetime curvature. We have already mentioned that, for a conformally coupled field, the stochastic source tensor must be traceless (up to first order perturbations around semiclassical gravity), in the sense that the stochastic variable $\xi_{\mu}^{\mu} \equiv \eta_{\mu \nu} \xi^{\mu \nu}$ behaves deterministically as a vanishing scalar field. This can be directly checked by noticing from Equations (102) and (115) that when $\Delta \xi=0$, one has $\left\langle\xi_{\mu}^{\mu}(x) \xi^{\alpha \beta}(y)\right\rangle_{\mathrm{s}}=0$, since $\mathcal{F}_{\mu}^{\mu}=3 \square$ and $\mathcal{F}^{\mu \alpha} \mathcal{F}_{\mu}^{\beta}=\square \mathcal{F}^{\alpha \beta}$. The Einstein-Langevin equations for this particular case (and generalized to a spatially-flat Robertson-Walker background) were first obtained in [73], where the coupling constant $\beta$ was fixed to be zero. See also [208] for a discussion of this result and its connection to the problem of structure formation in the trace anomaly driven inflation [162, 339, 355].

Note that the expectation value of the renormalized stress-energy tensor for a scalar field can be obtained by comparing Equation (118) with the Einstein-Langevin equation (15); its explicit expression is given in [259]. The results agree with the general form found by Horowitz [169, 170] using an axiomatic approach and coincide with that given in [110]. The particular cases of conformal coupling, $\Delta \xi=0$, and minimal coupling, $\Delta \xi=-1 / 6$, are also in agreement with the results for these cases given in $[72,169,170,223,340]$, modulo local terms proportional to $A^{(1) \mu \nu}$ and $B^{(1) \mu \nu}$ due to different choices of the renormalization scheme. For the case of a massive minimally-coupled scalar field, $\Delta \xi=-\frac{1}{6}$, our result is equivalent to that of [347].

\subsection{Correlation functions for gravitational perturbations}

Here we solve the Einstein-Langevin equations (118) for the components $G^{(1) \mu \nu}$ of the linearized Einstein tensor. Then we use these solutions to compute the corresponding two-point correlation functions, which give a measure of the gravitational fluctuations predicted by the stochastic semiclassical theory of gravity in the present case. Since the linearized Einstein tensor is invariant under gauge transformations of the metric perturbations, these two-point correlation functions are also gauge invariant. Once we have computed the two-point correlation functions for the linearized Einstein tensor, we find the solutions for the metric perturbations and compute the associated two-point correlation functions. The procedure used to solve the Einstein-Langevin equation is similar to the one used by Horowitz [169] (see also [110]) to analyze the stability of Minkowski spacetime in semiclassical gravity.

We first note that the tensors $A^{(1) \mu \nu}$ and $B^{(1) \mu \nu}$ can be written in terms of $G^{(1) \mu \nu}$ as

$$
A^{(1) \mu \nu}=\frac{2}{3}\left(\mathcal{F}^{\mu \nu} G_{\alpha}^{(1) \alpha}-\mathcal{F}_{\alpha}^{\alpha} G^{(1) \mu \nu}\right), \quad B^{(1) \mu \nu}=2 \mathcal{F}^{\mu \nu} G_{\alpha}^{(1) \alpha},
$$


where we have used $3 \square=\mathcal{F}_{\alpha}^{\alpha}$. Therefore, the Einstein-Langevin equation (118) can be seen as a linear integro-differential stochastic equation for the components $G^{(1) \mu \nu}$. In order to find solutions to Equation (118), it is convenient to Fourier transform it. With the convention $\tilde{f}(p)=$ $\int d^{4} x e^{-i p x} f(x)$ for a given field $f(x)$, one finds, from Equation (119),

$$
\begin{aligned}
& \tilde{A}^{(1) \mu \nu}(p)=2 p^{2} \tilde{G}^{(1) \mu \nu}(p)-\frac{2}{3} p^{2} P^{\mu \nu} \tilde{G}_{\alpha}^{(1) \alpha}(p), \\
& \tilde{B}^{(1) \mu \nu}(p)=-2 p^{2} P^{\mu \nu} \tilde{G}_{\alpha}^{(1) \alpha}(p) .
\end{aligned}
$$

The Fourier transform of the Einstein-Langevin Equation (118) now reads

$$
F_{\alpha \beta}^{\mu \nu}(p) \tilde{G}^{(1) \alpha \beta}(p)=8 \pi G \tilde{\xi}^{\mu \nu}(p),
$$

where

$$
F_{\alpha \beta}^{\mu \nu}(p) \equiv F_{1}(p) \delta_{(\alpha}^{\mu} \delta_{\beta)}^{\nu}+F_{2}(p) p^{2} P^{\mu \nu} \eta_{\alpha \beta},
$$

with

$$
\begin{aligned}
& F_{1}(p) \equiv 1+16 \pi G p^{2}\left[\frac{1}{4} \tilde{H}_{\mathrm{A}}(p)-2 \bar{\alpha}\right], \\
& F_{2}(p) \equiv-\frac{16}{3} \pi G\left[\frac{1}{4} \tilde{H}_{\mathrm{A}}(p)+\frac{3}{4} \tilde{H}_{\mathrm{B}}(p)-2 \bar{\alpha}-6 \bar{\beta}\right] .
\end{aligned}
$$

In the Fourier transformed Einstein-Langevin Equation (121), $\tilde{\xi}^{\mu \nu}(p)$, the Fourier transform of $\xi^{\mu \nu}(x)$, is a Gaussian stochastic source of zero average, and

$$
\left\langle\tilde{\xi}^{\mu \nu}(p) \tilde{\xi}^{\alpha \beta}\left(p^{\prime}\right)\right\rangle_{\mathrm{s}}=(2 \pi)^{4} \delta^{4}\left(p+p^{\prime}\right) \tilde{N}^{\mu \nu \alpha \beta}(p),
$$

where we have introduced the Fourier transform of the noise kernel. The explicit expression for $\tilde{N}^{\mu \nu \alpha \beta}(p)$ is found from Equations (101) and (102) to be

$$
\tilde{N}^{\mu \nu \alpha \beta}(p)=\frac{\theta\left(-p^{2}-4 m^{2}\right)}{720 \pi} \sqrt{1+4 \frac{m^{2}}{p^{2}}}\left[\frac{1}{4}\left(1+4 \frac{m^{2}}{p^{2}}\right)^{2}\left(p^{2}\right)^{2} P^{\mu \nu \alpha \beta}+10\left(3 \Delta \xi+\frac{m^{2}}{p^{2}}\right)^{2}\left(p^{2}\right)^{2} P^{\mu \nu} P^{\alpha \beta}\right],
$$

which in the massless case reduces to

$$
\lim _{m \rightarrow 0} \tilde{N}^{\mu \nu \alpha \beta}(p)=\frac{1}{480 \pi} \theta\left(-p^{2}\right)\left[\frac{1}{6}\left(p^{2}\right)^{2} P^{\mu \nu \alpha \beta}+60 \Delta \xi^{2}\left(p^{2}\right)^{2} P^{\mu \nu} P^{\alpha \beta}\right] .
$$

\subsubsection{Correlation functions for the linearized Einstein tensor}

In general we can write $G^{(1) \mu \nu}=\left\langle G^{(1) \mu \nu}\right\rangle_{\mathrm{s}}+G_{\mathrm{f}}^{(1) \mu \nu}$, where $G_{\mathrm{f}}^{(1) \mu \nu}$ is a solution to Equations (118) with zero average, or Equation (121) in the Fourier transformed version. The averages $\left\langle G^{(1) \mu \nu}\right\rangle_{\mathrm{S}}$ must be a solution of the linearized semiclassical Einstein equations obtained by averaging Equations (118) or (121). Solutions to these equations (especially in the massless case, $m=0$ ) have been studied by several authors [110, 152, 169, 170, 174, 223, 309, 310, 330, 344, 345], particularly in connection with the problem of the stability of the ground state of semiclassical gravity. The two-point correlation functions for the linearized Einstein tensor are defined by

$$
\begin{aligned}
\mathcal{G}^{\mu \nu \alpha \beta}\left(x, x^{\prime}\right) & \equiv\left\langle G^{(1) \mu \nu}(x) G^{(1) \alpha \beta}\left(x^{\prime}\right)\right\rangle_{\mathrm{s}}-\left\langle G^{(1) \mu \nu}(x)\right\rangle_{\mathrm{s}}\left\langle G^{(1) \alpha \beta}\left(x^{\prime}\right)\right\rangle_{\mathrm{s}} \\
& =\left\langle G_{\mathrm{f}}^{(1) \mu \nu}(x) G_{\mathrm{f}}^{(1) \alpha \beta}\left(x^{\prime}\right)\right\rangle_{\mathrm{s}} .
\end{aligned}
$$


Now we shall seek the family of solutions to the Einstein-Langevin equation, which can be written as a linear functional of the stochastic source, and whose Fourier transform $\tilde{G}^{(1) \mu \nu}(p)$ depends locally on $\tilde{\xi}^{\alpha \beta}(p)$. Each of these such solutions is a Gaussian stochastic field and thus can be completely characterized by the averages $\left\langle G^{(1) \mu \nu}\right\rangle_{\mathrm{s}}$ and the two-point correlation functions (127). For such a family of solutions, $\tilde{G}_{\mathrm{f}}^{(1) \mu \nu}(p)$ is the most general solution to Equation (121), which is linear, homogeneous, and local in $\tilde{\xi}^{\alpha \beta}(p)$. It can be written as

$$
\tilde{G}_{\mathrm{f}}^{(1) \mu \nu}(p)=8 \pi G D^{\mu \nu}{ }_{\alpha \beta}(p) \tilde{\xi}^{\alpha \beta}(p),
$$

where $D^{\mu \nu}{ }_{\alpha \beta}(p)$ are the components of a Lorentz-invariant tensor-field distribution in Minkowski spacetime (by "Lorentz-invariant" we mean invariant under transformations of the orthochronous Lorentz subgroup; see [169] for more details on the definition and properties of these tensor distributions). This tensor is symmetric under the interchanges of $\alpha \leftrightarrow \beta$ and $\mu \leftrightarrow \nu$, and is the most general solution of

$$
F_{\rho \sigma}^{\mu \nu}(p) D_{\alpha \beta}^{\rho \sigma}(p)=\delta_{(\alpha}^{\mu} \delta_{\beta)}^{\nu} .
$$

In addition, we must impose the conservation condition, $p_{\nu} \tilde{G}_{\mathrm{f}}^{(1) \mu \nu}(p)=0$, where this zero must be understood as a stochastic variable, which behaves deterministically as a zero vector field. We can write $D^{\mu \nu}{ }_{\alpha \beta}(p)=D_{\mathrm{p}}^{\mu \nu}{ }_{\alpha \beta}(p)+D_{\mathrm{h} \alpha \beta}^{\mu \nu}(p)$, where $D_{\mathrm{p}}^{\mu \nu}{ }_{\alpha \beta}(p)$ is a particular solution to Equation (129) and $D_{\mathrm{h} \quad \alpha \beta}^{\mu \nu}(p)$ is the most general solution to the homogeneous equation. Consequently, see Equation (128), we can write $\tilde{G}_{\mathrm{f}}^{(1) \mu \nu}(p)=\tilde{G}_{\mathrm{p}}^{(1) \mu \nu}(p)+\tilde{G}_{\mathrm{h}}^{(1) \mu \nu}(p)$. To find the particular solution, we try an ansatz of the form

$$
D_{\mathrm{p} \alpha \beta}^{\mu \nu}(p)=d_{1}(p) \delta_{(\alpha}^{\mu} \delta_{\beta)}^{\nu}+d_{2}(p) p^{2} P^{\mu \nu} \eta_{\alpha \beta}
$$

Substituting this ansatz into Equations (129), it is easy to see that it solves these equations if we take

with

$$
d_{1}(p)=\left[\frac{1}{F_{1}(p)}\right]_{\mathrm{r}}, \quad d_{2}(p)=-\left[\frac{F_{2}(p)}{F_{1}(p) F_{3}(p)}\right]_{\mathrm{r}},
$$

$$
F_{3}(p) \equiv F_{1}(p)+3 p^{2} F_{2}(p)=1-48 \pi G p^{2}\left[\frac{1}{4} \tilde{H}_{\mathrm{B}}(p)-2 \bar{\beta}\right],
$$

and where the notation [ $]_{\mathrm{r}}$ means that the zeros of the denominators are regulated with appropriate prescriptions in such a way that $d_{1}(p)$ and $d_{2}(p)$ are well-defined Lorentz-invariant scalar distributions. This yields a particular solution to the Einstein-Langevin equations,

$$
\tilde{G}_{\mathrm{p}}^{(1) \mu \nu}(p)=8 \pi G D_{\mathrm{p} \alpha \beta}^{\mu \nu}(p) \tilde{\xi}^{\alpha \beta}(p),
$$

which, since the stochastic source is conserved, satisfies the conservation condition. Note that, in the case of a massless scalar field $(m=0)$, the above solution has a functional form analogous to that of the solutions of linearized semiclassical gravity found in the appendix of [110]. Notice also that, for a massless conformally-coupled field ( $m=0$ and $\Delta \xi=0)$, the second term on the right-hand side of Equation (130) will not contribute in the correlation functions (127), since in this case the stochastic source is traceless.

A detailed analysis given in [259] concludes that the homogeneous solution $\tilde{G}_{\mathrm{h}}^{(1) \mu \nu}(p)$ gives no contribution to the correlation functions (127). Consequently $\mathcal{G}^{\mu \nu \alpha \beta}\left(x, x^{\prime}\right)=\left\langle G_{\mathrm{p}}^{(1) \mu \nu}(x) G_{\mathrm{p}}^{(1) \alpha \beta}\left(x^{\prime}\right)\right\rangle_{\mathrm{s}}$, where $G_{\mathrm{p}}^{(1) \mu \nu}(x)$ is the inverse Fourier transform of Equation (133), and the correlation functions (127) are

$$
\left\langle\tilde{G}_{\mathrm{p}}^{(1) \mu \nu}(p) \tilde{G}_{\mathrm{p}}^{(1) \alpha \beta}\left(p^{\prime}\right)\right\rangle_{\mathrm{s}}=64(2 \pi)^{6} G^{2} \delta^{4}\left(p+p^{\prime}\right) D_{\mathrm{p} \rho}^{\mu \nu} \rho(p) D_{\mathrm{p}}^{\alpha \beta}{ }_{\lambda \gamma}(-p) \tilde{N}^{\rho \sigma \lambda \gamma}(p) .
$$


It is easy to see from the above analysis that the prescriptions [ $]_{\mathrm{r}}$ in the factors $D_{\mathrm{p}}$ are irrelevant in the last expression and thus can be suppressed. Taking into account that $F_{l}(-p)=F_{l}^{*}(p)$, with $l=1,2,3$, we get from Equations (130) and (131)

$$
\begin{aligned}
\left\langle\tilde{G}_{\mathrm{p}}^{(1) \mu \nu}(p) \tilde{G}_{\mathrm{p}}^{(1) \alpha \beta}\left(p^{\prime}\right)\right\rangle_{\mathrm{s}}=64(2 \pi)^{6} G^{2} \frac{\delta^{4}\left(p+p^{\prime}\right)}{\left|F_{1}(p)\right|^{2}}\left[\tilde{N}^{\mu \nu \alpha \beta}(p)-\frac{F_{2}(p)}{F_{3}(p)} p^{2} P^{\mu \nu} \tilde{N}^{\alpha \beta \rho}{ }_{\rho}(p)\right. \\
\left.-\frac{F_{2}^{*}(p)}{F_{3}^{*}(p)} p^{2} P^{\alpha \beta} \tilde{N}^{\mu \nu \rho}(p)+\frac{\left|F_{2}(p)\right|^{2}}{\left|F_{3}(p)\right|^{2}} p^{2} P^{\mu \nu} p^{2} P^{\alpha \beta} \tilde{N}_{\rho}^{\rho}{ }_{\rho}{ }_{\sigma}(p)\right] .
\end{aligned}
$$

This last expression is well-defined as a bi-distribution and can be easily evaluated using Equation (125). The final explicit result for the Fourier-transformed correlation function for the Einstein tensor is thus

$$
\begin{aligned}
\left\langle\tilde{G}_{\mathrm{p}}^{(1) \mu \nu}(p) \tilde{G}_{\mathrm{p}}^{(1) \alpha \beta}\left(p^{\prime}\right)\right\rangle_{\mathrm{s}}= & \frac{2}{45}(2 \pi)^{5} G^{2} \frac{\delta^{4}\left(p+p^{\prime}\right)}{\left|F_{1}(p)\right|^{2}} \theta\left(-p^{2}-4 m^{2}\right) \sqrt{1+4 \frac{m^{2}}{p^{2}}} \\
\times & {\left[\frac{1}{4}\left(1+4 \frac{m^{2}}{p^{2}}\right)^{2}\left(p^{2}\right)^{2} P^{\mu \nu \alpha \beta}\right.} \\
& \left.+10\left(3 \Delta \xi+\frac{m^{2}}{p^{2}}\right)^{2}\left(p^{2}\right)^{2} P^{\mu \nu} P^{\alpha \beta}\left|1-3 p^{2} \frac{F_{2}(p)}{F_{3}(p)}\right|^{2}\right] .
\end{aligned}
$$

To obtain the correlation functions in coordinate space, Equation (127), we take the inverse Fourier transform. The final result is

$$
\mathcal{G}^{\mu \nu \alpha \beta}\left(x, x^{\prime}\right)=\frac{\pi}{45} G^{2} \mathcal{F}_{x}^{\mu \nu \alpha \beta} \mathcal{G}_{\mathrm{A}}\left(x-x^{\prime}\right)+\frac{8 \pi}{9} G^{2} \mathcal{F}_{x}^{\mu \nu} \mathcal{F}_{x}^{\alpha \beta} \mathcal{G}_{\mathrm{B}}\left(x-x^{\prime}\right),
$$

with

$$
\begin{aligned}
& \tilde{\mathcal{G}}_{\mathrm{A}}(p) \equiv \theta\left(-p^{2}-4 m^{2}\right) \sqrt{1+4 \frac{m^{2}}{p^{2}}}\left(1+4 \frac{m^{2}}{p^{2}}\right)^{2} \frac{1}{\left|F_{1}(p)\right|^{2}}, \\
& \tilde{\mathcal{G}}_{\mathrm{B}}(p) \equiv \theta\left(-p^{2}-4 m^{2}\right) \sqrt{1+4 \frac{m^{2}}{p^{2}}}\left(3 \Delta \xi+\frac{m^{2}}{p^{2}}\right)^{2} \frac{1}{\left|F_{1}(p)\right|^{2}}\left|1-3 p^{2} \frac{F_{2}(p)}{F_{3}(p)}\right|^{2},
\end{aligned}
$$

where $F_{l}(p), l=1,2,3$, are given in Equations (123) and (132). Notice that, for a massless field $(m=0)$, we have

$$
\begin{aligned}
& F_{1}(p)=1+4 \pi G p^{2} \tilde{H}\left(p ; \bar{\mu}^{2}\right), \\
& F_{2}(p)=-\frac{16}{3} \pi G\left[\left(1+180 \Delta \xi^{2}\right) \frac{1}{4} \tilde{H}\left(p ; \bar{\mu}^{2}\right)-6 \Upsilon\right], \\
& F_{3}(p)=1-48 \pi G p^{2}\left[15 \Delta \xi^{2} \tilde{H}\left(p ; \bar{\mu}^{2}\right)-2 \Upsilon\right],
\end{aligned}
$$

with $\bar{\mu} \equiv \mu \exp \left(1920 \pi^{2} \bar{\alpha}\right)$ and $\Upsilon \equiv \bar{\beta}-60 \Delta \xi^{2} \bar{\alpha}$, and where $\tilde{H}\left(p ; \mu^{2}\right)$ is the Fourier transform of $H\left(x ; \mu^{2}\right)$ given in Equation (117).

\subsubsection{Correlation functions for the metric perturbations}

Starting from the solutions found for the linearized Einstein tensor, which are characterized by the two-point correlation functions (137) (or, in terms of Fourier transforms, Equation (136)), we can 
now solve the equations for the metric perturbations. Working in the harmonic gauge, $\partial_{\nu} \bar{h}^{\mu \nu}=0$ (this zero must be understood in a statistical sense), where $\bar{h}_{\mu \nu} \equiv h_{\mu \nu}-\frac{1}{2} \eta_{\mu \nu} h_{\alpha}^{\alpha}$, the equations for the metric perturbations in terms of the Einstein tensor are

$$
\square \bar{h}^{\mu \nu}(x)=-2 G^{(1) \mu \nu}(x),
$$

or, in terms of Fourier transforms, $p^{2} \tilde{\bar{h}}^{\mu \nu}(p)=2 \tilde{G}^{(1) \mu \nu}(p)$. Similarly to the analysis of the equation for the Einstein tensor, we can write $\bar{h}^{\mu \nu}=\left\langle\bar{h}^{\mu \nu}\right\rangle_{\mathrm{s}}+\bar{h}_{\mathrm{f}}^{\mu \nu}$, where $\bar{h}_{\mathrm{f}}^{\mu \nu}$ is a solution to these equations with zero average, and the two-point correlation functions are defined by

$$
\begin{aligned}
\mathcal{H}^{\mu \nu \alpha \beta}\left(x, x^{\prime}\right) & \equiv\left\langle\bar{h}^{\mu \nu}(x) \bar{h}^{\alpha \beta}\left(x^{\prime}\right)\right\rangle_{\mathrm{s}}-\left\langle\bar{h}^{\mu \nu}(x)\right\rangle_{\mathrm{s}}\left\langle\bar{h}^{\alpha \beta}\left(x^{\prime}\right)\right\rangle_{\mathrm{s}} \\
& =\left\langle\bar{h}_{\mathrm{f}}^{\mu \nu}(x) \bar{h}_{\mathrm{f}}^{\alpha \beta}\left(x^{\prime}\right)\right\rangle_{\mathrm{s}}
\end{aligned}
$$

We can now seek solutions of the Fourier transform of Equation (140) of the form $\tilde{\bar{h}}_{\mathrm{f}}^{\mu \nu}(p)=$ $2 D(p) \tilde{G}_{\mathrm{f}}^{(1) \mu \nu}(p)$, where $D(p)$ is a Lorentz-invariant scalar distribution in Minkowski spacetime, which is the most general solution of $p^{2} D(p)=1$. Note that, since the linearized Einstein tensor is conserved, solutions of this form automatically satisfy the harmonic gauge condition. As in Section 6.4.1 we can write $D(p)=\left[1 / p^{2}\right]_{\mathrm{r}}+D_{\mathrm{h}}(p)$, where $D_{\mathrm{h}}(p)$ is the most general solution to the associated homogeneous equation and, correspondingly, we have $\tilde{\bar{h}}_{\mathrm{f}}^{\mu \nu}(p)=\tilde{\bar{h}}_{\mathrm{p}}^{\mu \nu}(p)+\tilde{\bar{h}}_{\mathrm{h}}^{\mu \nu}(p)$. However, since $D_{\mathrm{h}}(p)$ has support on the set of points for which $p^{2}=0$, it is easy to see from Equation (136) (from the factor $\theta\left(-p^{2}-4 m^{2}\right)$ ) that $\left\langle\tilde{\bar{h}}_{\mathrm{h}}^{\mu \nu}(p) \tilde{G}_{\mathrm{f}}^{(1) \alpha \beta}\left(p^{\prime}\right)\right\rangle_{\mathrm{s}}=0$ and, thus, the twopoint correlation functions (141) can be computed from $\left\langle\tilde{\bar{h}}_{\mathrm{f}}^{\mu \nu}(p) \tilde{\bar{h}}_{\mathrm{f}}^{\alpha \beta}\left(p^{\prime}\right)\right\rangle_{\mathrm{s}}=\left\langle\tilde{\bar{h}}_{\mathrm{p}}^{\mu \nu}(p) \tilde{\bar{h}}_{\mathrm{p}}^{\alpha \beta}\left(p^{\prime}\right)\right\rangle_{\mathrm{s}}$. From Equation (136), and due to the factor $\theta\left(-p^{2}-4 m^{2}\right)$, it is also easy to see that the prescription [ $]_{\mathrm{r}}$ is irrelevant in this correlation function, and we obtain

$$
\left\langle\tilde{\bar{h}}_{\mathrm{p}}^{\mu \nu}(p) \tilde{\bar{h}}_{\mathrm{p}}^{\alpha \beta}\left(p^{\prime}\right)\right\rangle_{\mathrm{s}}=\frac{4}{\left(p^{2}\right)^{2}}\left\langle\tilde{G}_{\mathrm{p}}^{(1) \mu \nu}(p) \tilde{G}_{\mathrm{p}}^{(1) \alpha \beta}\left(p^{\prime}\right)\right\rangle_{\mathrm{s}}
$$

where $\left\langle\tilde{G}_{\mathrm{p}}^{(1) \mu \nu}(p) \tilde{G}_{\mathrm{p}}^{(1) \alpha \beta}\left(p^{\prime}\right)\right\rangle_{\mathrm{s}}$ is given by Equation (136). The right-hand side of this equation is a well-defined bi-distribution, at least for $m \neq 0$ (the $\theta$ function provides the suitable cutoff). In the massless field case, since the noise kernel is obtained as the limit $m \rightarrow 0$ of the noise kernel for a massive field, it seems that the natural prescription to avoid divergences on the lightcone $p^{2}=0$ is a Hadamard finite part (see $[322,388]$ for its definition). Taking this prescription, we also get a well-defined bi-distribution for the massless limit of the last expression.

The final result for the two-point correlation function for the field $\bar{h}^{\mu \nu}$ is

$$
\mathcal{H}^{\mu \nu \alpha \beta}\left(x, x^{\prime}\right)=\frac{4 \pi}{45} G^{2} \mathcal{F}_{x}^{\mu \nu \alpha \beta} \mathcal{H}_{\mathrm{A}}\left(x-x^{\prime}\right)+\frac{32 \pi}{9} G^{2} \mathcal{F}_{x}^{\mu \nu} \mathcal{F}_{x}^{\alpha \beta} \mathcal{H}_{\mathrm{B}}\left(x-x^{\prime}\right),
$$

where $\tilde{\mathcal{H}}_{\mathrm{A}}(p) \equiv\left[1 /\left(p^{2}\right)^{2}\right] \tilde{\mathcal{G}}_{\mathrm{A}}(p)$ and $\tilde{\mathcal{H}}_{\mathrm{B}}(p) \equiv\left[1 /\left(p^{2}\right)^{2}\right] \tilde{\mathcal{G}}_{\mathrm{B}}(p)$, with $\tilde{\mathcal{G}}_{\mathrm{A}}(p)$ and $\tilde{\mathcal{G}}_{\mathrm{B}}(p)$ given by Equation (138). The two-point correlation functions for the metric perturbations can be easily obtained using $h_{\mu \nu}=\bar{h}_{\mu \nu}-\frac{1}{2} \eta_{\mu \nu} \bar{h}_{\alpha}^{\alpha}$.

\subsubsection{Conformally-coupled field}

For a conformally coupled field, i.e., when $m=0$ and $\Delta \xi=0$, the previous correlation functions are greatly simplified and can be approximated explicitly in terms of analytic functions. The detailed results are given in [259]; here we outline the main features.

When $m=0$ and $\Delta \xi=0$, we have $\mathcal{G}_{\mathrm{B}}(x)=0$ and $\tilde{\mathcal{G}}_{\mathrm{A}}(p)=\theta\left(-p^{2}\right)\left|F_{1}(p)\right|^{-2}$. Thus the two-point correlations functions for the Einstein tensor are written

$$
\mathcal{G}^{\mu \nu \alpha \beta}\left(x, x^{\prime}\right)=\frac{\pi}{45} G^{2} \mathcal{F}_{x}^{\mu \nu \alpha \beta} \int \frac{d^{4} p}{(2 \pi)^{4}} \frac{e^{i p\left(x-x^{\prime}\right)} \theta\left(-p^{2}\right)}{\left|1+4 \pi G p^{2} \tilde{H}\left(p ; \bar{\mu}^{2}\right)\right|^{2}},
$$


where $\tilde{H}\left(p ; \bar{\mu}^{2}\right)=\left(480 \pi^{2}\right)^{-1} \ln \left[-\left(\left(p^{0}+i \epsilon\right)^{2}+p^{i} p_{i}\right) / \mu^{2}\right]$; (see Equation (117)).

To estimate this integral, let us consider spacelike separated points $\left(x-x^{\prime}\right)^{\mu}=\left(0, \mathbf{x}-\mathbf{x}^{\prime}\right)$, and define $\mathbf{y}=\mathbf{x}-\mathbf{x}^{\prime}$. We may now formally change the momentum variable $p^{\mu}$ by the dimensionless vector $s^{\mu}, p^{\mu}=s^{\mu} /|\mathbf{y}|$. Then the previous integral denominator is $\left|1+16 \pi\left(L_{\mathrm{P}} /|\mathbf{y}|\right)^{2} s^{2} \tilde{H}(s)\right|^{2}$, where we have introduced the Planck length $L_{\mathrm{P}}=\sqrt{G}$. It is clear that we can consider two regimes: (a) when $L_{\mathrm{P}} \ll|\mathbf{y}|$, and (b) when $|\mathbf{y}| \sim L_{\mathrm{P}}$. In case (a) the correlation function, for the 0000 component, say, will be of the order

$$
\mathcal{G}^{0000}(\mathbf{y}) \sim \frac{L_{\mathrm{P}}^{4}}{|\mathbf{y}|^{8}} .
$$

In case (b), when the denominator has zeros, a detailed calculation carried out in [259] shows that

$$
\mathcal{G}^{0000}(\mathbf{y}) \sim e^{-|\mathbf{y}| / L_{\mathrm{P}}}\left(\frac{L_{\mathrm{P}}}{|\mathbf{y}|^{5}}+\cdots+\frac{1}{L_{\mathrm{P}}^{2}|\mathbf{y}|^{2}}\right),
$$

which indicates an exponential decay at distances around the Planck scale. Thus short scale fluctuations are strongly suppressed.

For the two-point metric correlation the results are similar. In this case we have

$$
\mathcal{H}^{\mu \nu \alpha \beta}\left(x, x^{\prime}\right)=\frac{4 \pi}{45} G^{2} \mathcal{F}_{x}^{\mu \nu \alpha \beta} \int \frac{d^{4} p}{(2 \pi)^{4}} \frac{e^{i p\left(x-x^{\prime}\right)} \theta\left(-p^{2}\right)}{\left(p^{2}\right)^{2}\left|1+4 \pi G p^{2} \tilde{H}\left(p ; \bar{\mu}^{2}\right)\right|^{2}} .
$$

The integrand has the same behavior as the correlation function of Equation (144), thus matter fields tends to suppress the short-scale metric perturbations. In this case we find, as for the correlation of the Einstein tensor, that for case (a) above we have

$$
\mathcal{H}^{0000}(\mathbf{y}) \sim \frac{L_{\mathrm{P}}^{4}}{|\mathbf{y}|^{4}}
$$

and for case (b) we have

$$
\mathcal{H}^{0000}(\mathbf{y}) \sim e^{-|\mathbf{y}| / L_{\mathrm{P}}}\left(\frac{L_{\mathrm{P}}}{|\mathbf{y}|}+\ldots\right) .
$$

It is interesting to write expression (145) in an alternative way. If we use the dimensionless tensor $P^{\mu \nu \alpha \beta}$ introduced in Equation (107), which accounts for the effect of the operator $\mathcal{F}_{x}^{\mu \nu \alpha \beta}$, we can write

$$
\mathcal{H}^{\mu \nu \alpha \beta}\left(x, x^{\prime}\right)=\frac{4 \pi}{45} G^{2} \int \frac{d^{4} p}{(2 \pi)^{4}} \frac{e^{i p\left(x-x^{\prime}\right)} P^{\mu \nu \alpha \beta} \theta\left(-p^{2}\right)}{\left|1+4 \pi G p^{2} \tilde{H}\left(p ; \bar{\mu}^{2}\right)\right|^{2}} .
$$

This expression allows a direct comparison with the graviton propagator for linearized quantum gravity in the $1 / N$ expansion found by Tomboulis [348]. One can see that the imaginary part of the graviton propagator leads, in fact, to Equation (146). In [317] it is shown that the two-point correlation functions for the metric perturbations derived from the Einstein-Langevin equation are equivalent to the symmetrized quantum two-point correlation functions for the metric fluctuations in the large $N$ expansion of quantum gravity interacting with $N$ matter fields.

\subsection{Stability of Minkowski spacetime}

In this section we apply the validity criterion for semiclassical gravity introduced in Section 3.3 to flat spacetime. The Minkowski metric is a particularly simple and interesting solution of semiclassical gravity. In fact, as we have seen in Section 6.1, when the quantum fields are in the Minkowski 
vacuum state, one may take the renormalized expectation value of the stress tensor as $\left\langle\hat{T}_{a b}^{R}[\eta]\right\rangle=0$; this is equivalent to assuming that the cosmological constant is zero. Then the Minkowski metric $\eta_{a b}$ is a solution of the semiclassical Einstein equation (8). Thus, we can look for the stability of Minkowski spacetime against quantum matter fields. According to the criteria we have established, we have to look for the behavior of the two-point quantum correlations for the metric perturbations $h_{a b}(x)$ over the Minkowski background, which are given by Equations (16) and (17). As we have emphasized before, these metric fluctuations separate in two parts: the first term on the right-hand side of Equation (17), which corresponds to the intrinsic metric fluctuations, and the second term, which corresponds to the induced metric fluctuations.

\subsubsection{Intrinsic metric fluctuations}

Let us first consider the intrinsic metric fluctuations,

$$
\left\langle h_{a b}(x) h_{c d}(y)\right\rangle_{\mathrm{int}}=\left\langle h_{a b}^{0}(x) h_{c d}^{0}(y)\right\rangle_{s}
$$

where $h_{a b}^{0}$ are the homogeneous solutions of the Einstein-Langevin equation (15), or equivalently the linearly-perturbed semiclassical equation, and where the statistical average is taken with respect to the Wigner distribution that describes the initial quantum state of the metric perturbations. Since these solutions are described by the linearized semiclassical equation around flat spacetime, we can make use of the results derived in $[10,11,110,169]$. The solutions for the case of a massless scalar field were first discussed in [169] and an exhaustive description can be found in Appendix A of [110]. It is convenient to decompose the perturbation around Minkowski spacetime into scalar, vectorial and tensorial parts, as

$$
h_{a b}=\bar{\phi} \eta_{a b}+\left(\nabla_{(a} \nabla_{b)}-\eta_{a b} \nabla_{c} \nabla^{c}\right) \psi+2 \nabla_{(a} v_{b)}+h_{a b}^{\mathrm{TT}},
$$

where $v^{a}$ is a transverse vector and $h_{a b}^{\mathrm{TT}}$ is a transverse and traceless symmetric tensor, i.e., $\nabla_{a} v^{a}=0, \nabla^{a} h_{a b}^{\mathrm{TT}}=0$ and $\left(h^{\mathrm{TT}}\right)_{a}^{a}=0$. A vector field $\zeta^{a}$ characterizes the gauge freedom due to infinitesimal diffeomorphisms as $h_{a b} \rightarrow h_{a b}+\nabla_{a} \zeta_{b}+\nabla_{b} \zeta_{a}$. We may use this freedom to choose a gauge; a convenient election is the Lorentz or harmonic gauge defined as

$$
\nabla^{a}\left(h_{a b}-\frac{1}{2} \eta_{a b} h_{c}^{c}\right)=0
$$

When this gauge is imposed we have the following conditions on the metric perturbations $\nabla_{a} \nabla^{a} v^{b}=$ 0 and $\nabla_{b} \bar{\phi}=0$, which implies $\bar{\phi}=$ const. A remaining gauge freedom compatible with the Lorentz gauge is still possible provided the vector field $\zeta^{a}$ satisfies the condition $\nabla_{a} \nabla^{a} \zeta^{b}=0$. One can easily see [203] that the vectorial and scalar part $\bar{\phi}$ can be eliminated, as well as the contribution of the scalar part $\psi$, which corresponds to Fourier modes $\tilde{\psi}(p)$ with $p^{2}=0$. Thus, we will assume that we impose the Lorentz gauge with additional gauge transformations, which leave only the tensorial component and the modes of the scalar component $\psi$ with $p^{2} \neq 0$ in Fourier space.

Using the metric decomposition (148) we may compute the linearized Einstein tensor $G_{a b}^{(1)}$. It is found that the vectorial part of the metric perturbation gives no contribution to this tensor, and the scalar and tensorial components give rise, respectively, to scalar and tensorial components: $G_{a b}^{(1)(S)}$ and $G_{a b}^{(1)(T)}$. Thus, let us now write the Fourier transform of the homogeneous Einstein-Langevin equation (121), which is equivalent to the linearized semiclassical Einstein equation,

$$
F_{\alpha \beta}^{\mu \nu}(p) \tilde{G}^{(1) \alpha \beta}(p)=0 .
$$


Using the previous decomposition of the Einstein tensor this equation can be re-written in terms of its scalar and tensorial parts as

$$
\begin{aligned}
{\left[F_{1}(p)+3 p^{2} F_{2}(p)\right] \tilde{G}_{\mu \nu}^{(1)(\mathrm{S})}(p) } & =0, \\
F_{1}(p) \tilde{G}_{\mu \nu}^{(1)(\mathrm{T})}(p) & =0 .
\end{aligned}
$$

where $F_{1}(p)$ and $F_{2}(p)$ are given by Equations $(123)$, and $\tilde{G}_{\mu \nu}^{(1)(\mathrm{S})}$ and $\tilde{G}_{\mu \nu}^{(1)(\mathrm{T})}$ denote, respectively, the Fourier-transformed scalar and tensorial parts of the linearized Einstein tensor. To simplify the problem and to illustrate, in particular, how the runaway solutions arise, we will consider the case of a massless and conformally coupled field (see [110] for the massless case with arbitrary coupling and $[10,259]$ for the general massive case). Thus substituting $m=0$ and $\xi=1 / 6$ into the functions $F_{1}(p)$ and $F_{2}(p)$, and using Equation (117), the above equations become

$$
\begin{aligned}
& \left(1+12 \kappa \bar{\beta} p^{2}\right) \tilde{G}_{\mu \nu}^{(1)(\mathrm{S})}(p)=0, \\
& \lim _{\epsilon \rightarrow 0^{+}}\left[1+\frac{\kappa p^{2}}{960 \pi^{2}} \ln \left(\frac{-\left(p^{0}+i \epsilon\right)^{2}+\vec{p}^{2}}{\mu^{2}}\right)\right] \tilde{G}_{\mu \nu}^{(1)(\mathrm{T})}(p)=0,
\end{aligned}
$$

where $\kappa=8 \pi G$. Let us consider these two equations separately.

For the scalar component when $\bar{\beta}=0$ the only solution is $\tilde{G}_{\mu \nu}^{(1)(\mathrm{S})}(p)=0$. When $\bar{\beta}>0$ the solutions for the scalar component exhibit an oscillatory behavior in spacetime coordinates, which corresponds to a massive scalar field with $m^{2}=(12 \kappa|\bar{\beta}|)^{-1}$; for $\bar{\beta}<0$ the solutions correspond to a tachyonic field with $m^{2}=-(12 \kappa|\bar{\beta}|)^{-1}$. In spacetime coordinates they exhibit an exponential behavior in time, growing or decreasing, for wavelengths larger than $4 \pi(3 \kappa|\bar{\beta}|)^{1 / 2}$ and an oscillatory behavior for wavelengths smaller than $4 \pi(3 \kappa|\bar{\beta}|)^{1 / 2}$. On the other hand, the solution $\tilde{G}_{\mu \nu}^{(1)(\mathrm{S})}(p)=0$ is completely trivial since any scalar metric perturbation $\tilde{h}_{\mu \nu}(p)$ giving rise to a vanishing linearized Einstein tensor can be eliminated by a gauge transformation.

For the tensorial component, when $\mu \leq \mu_{\text {crit }}=l_{p}^{-1}(120 \pi)^{1 / 2} e^{\gamma}$, where $l_{p}$ is the Planck length $\left(l_{p}^{2} \equiv \kappa / 8 \pi\right)$, the first factor in Equation (154) vanishes for four complex values of $p^{0}$ of the form $\pm \omega$ and $\pm \omega^{*}$, where $\omega$ is some complex value. This means that, in the corresponding propagator, there are two poles on the upper half-plane of the complex $p^{0}$ plane and two poles in the lower half-plane. We will consider here the case in which $\mu<\mu_{\text {crit }}$; a detailed description of the situation for $\mu \geq \mu_{\text {crit }}$ can be found in Appendix A of [110]. The two zeros on the upper half of the complex plane correspond to solutions in spacetime coordinates, which exponentially grow in time, whereas the two on the lower half correspond to solutions exponentially decreasing in time. Strictly speaking, these solutions only exist in spacetime coordinates, since their Fourier transform is not well-defined. They are commonly referred to as runaway solutions and for $\mu \sim l_{p}^{-1}$ they grow exponentially in time scales comparable to the Planck time.

Consequently, in addition to the solutions with $G_{a b}^{(1)}(x)=0$, there are other solutions that in Fourier space take the form $\tilde{G}_{a b}^{(1)}(p) \propto \delta\left(p^{2}-p_{0}^{2}\right)$ for some particular values of $p_{0}$, but all of them exhibit exponential instabilities with characteristic Planckian time scales. In order to deal with those unstable solutions, one possibility is to make use of the order-reduction prescription [295], which we will briefly summarize in Section 6.5.3. Note that the $p^{2}$ terms in Equations (153) and (154) come from two spacetime derivatives of the Einstein tensor, moreover, the $p^{2} \ln p^{2}$ term comes from the nonlocal term of the expectation value of the stress tensor. The order-reduction prescription amounts here to neglecting these higher derivative terms. Thus, neglecting the terms proportional to $p^{2}$ in Equations (153) and (154), we are left with only the solutions, which satisfy $\tilde{G}_{a b}^{(1)}(p)=0$. The result for the metric perturbation in the gauge introduced above can be obtained 
by solving for the Einstein tensor, which in the Lorentz gauge of Equation (149) reads:

$$
\tilde{G}_{a b}^{(1)}(p)=\frac{1}{2} p^{2}\left(\tilde{h}_{a b}(p)-\frac{1}{2} \eta_{a b} \tilde{h}_{c}^{c}(p)\right)
$$

These solutions for $\tilde{h}_{a b}(p)$ simply correspond to free linear gravitational waves propagating in Minkowski spacetime expressed in the transverse and traceless (TT) gauge. When substituting back into Equation (147) and averaging over the initial conditions we simply get the symmetrized quantum correlation function for free gravitons in the TT gauge for the state given by the Wigner distribution. As far as the intrinsic fluctuations are concerned, it seems that the order-reduction prescription is too drastic, at least in the case of Minkowski spacetime, since no effects due to the interaction with the quantum matter fields are left.

A second possibility, proposed by Hawking et al. [161, 162], is to impose boundary conditions, which discard the runaway solutions that grow unbounded in time. These boundary conditions correspond to a special prescription for the integration contour when Fourier transforming back to spacetime coordinates. As we will discuss in more detail in Section 6.5.2, this prescription reduces here to integrating along the real axis in the $p^{0}$ complex plane. Following that procedure we get, for example, that for a massless conformally-coupled matter field with $\bar{\beta}>0$ the intrinsic contribution to the symmetrized quantum correlation function coincides with that of free gravitons plus an extra contribution for the scalar part of the metric perturbations. This extra-massive scalar renders Minkowski spacetime stable, but also plays a crucial role in providing a graceful exit in inflationary models driven by the vacuum polarization of a large number of conformal fields. Such a massive scalar field would not be in conflict with present observations because, for the range of parameters considered, the mass would be far too large to have observational consequences [162].

\subsubsection{Induced metric fluctuations}

Induced metric fluctuations are described by the second term in Equation (17). They are dependent on the noise kernel that describes the stress-tensor fluctuations of the matter fields,

$$
\left\langle h_{a b}(x) h_{c d}(y)\right\rangle_{\mathrm{ind}}=\frac{\bar{\kappa}^{2}}{N} \int d^{4} x^{\prime} d^{4} y^{\prime} \sqrt{g\left(x^{\prime}\right) g\left(y^{\prime}\right)} G_{a b e f}^{\mathrm{ret}}\left(x, x^{\prime}\right) N^{e f g h}\left(x^{\prime}, y^{\prime}\right) G_{c d g h}^{\mathrm{ret}}\left(y, y^{\prime}\right)
$$

where here we have written the expression in the large $N$ limit, so that $\bar{\kappa}=N \kappa$, where $\kappa=8 \pi G$ and $N$ is the number of independent free scalar fields. The contribution corresponding to the induced quantum fluctuations is equivalent to the stochastic correlation function obtained by considering just the inhomogeneous part of the solution to the Einstein-Langevin equation. We can make use of the results for the metric correlations obtained in Sections 6.3 and 6.4 for solving the EinsteinLangevin equation. In fact, one should simply take $N=1$ to transform our expressions here to those of Sections 6.3 and 6.4 or, more precisely, one should multiply the noise kernel in these expressions by $N$ in order to use those expressions here, as follows from the fact that we now have $N$ independent matter fields.

As we have seen in Section 6.4, following [259], the Einstein-Langevin equation can be entirely written in terms of the linearized Einstein tensor. The equation involves second spacetime derivatives of that tensor and, in terms of its Fourier components, is given in Equation (121) as

$$
F_{\alpha \beta}^{\mu \nu}(p) \tilde{G}^{(1) \alpha \beta}(p)=\bar{\kappa} \tilde{\xi}^{\mu \nu}(p),
$$

where we have now used the rescaled coupling $\bar{\kappa}$. The solution for the linearized Einstein tensor is given in Equation (133) in terms of the retarded propagator $D_{\mu \nu \rho \sigma}(p)$ defined in Equation (129). Now this propagator, which is written in Equation (130), exhibits two poles in the upper half complex $p^{0}$ plane and two poles in the lower half-plane, as we have seen analyzing the zeros in 
Equations (153) and (154) for the massless and conformally coupled case. The retarded propagator in spacetime coordinates is obtained, as usual, by taking the appropriate integration contour in the $p^{0}$ plane. It is convenient in this case to deform the integration path along the real $p^{0}$ axis so as to leave the two poles of the upper half-plane below that path. In this way, when closing the contour by an upper half-circle, in order to compute the anti-causal part of the propagator, there will be no contribution. The problem now is that when closing the contour on the lower half-plane, in order to compute the causal part, the contribution of the upper half-plane poles gives an unbounded solution, a runaway instability. If we adopt the Hawking et al. [161, 162] criterion of imposing final boundary conditions, which discard solutions growing unboundedly in time, this implies that we just need to take the integral along the real axis, as was done in Section 6.4.2. But now that the propagator is no longer strictly retarded, there are causality violations in time scales on the order of $\sqrt{N} l_{p}$, which should have no observable consequences. This propagator, however, has a well-defined Fourier transform.

Following the steps after Equation (133), the Fourier transform of the two-point correlation for the linearized Einstein tensor can be written in our case as,

$$
\left\langle\tilde{G}_{\mu \nu}^{(1)}(p) \tilde{G}_{\alpha \beta}^{(1)}\left(p^{\prime}\right)\right\rangle_{\mathrm{ind}}=\frac{\bar{\kappa}^{2}}{N}(2 \pi)^{4} \delta^{4}\left(p+p^{\prime}\right) D_{\mu \nu \rho \sigma}(p) D_{\alpha \beta \lambda \gamma}(-p) \tilde{N}^{\rho \sigma \lambda \gamma}(p),
$$

where the noise kernel $\tilde{N}^{\rho \sigma \lambda \gamma}(p)$ is given by Equation (125). Note that these correlation functions are invariant under gauge transformations of the metric perturbations because the linearized Einstein tensor is invariant under those transformations.

We may also use the order-reduction prescription, which amounts in this case to neglecting terms in the propagator, which are proportional to $p^{2}$, corresponding to two spacetime derivatives of the Einstein tensor. The propagator then becomes a constant, and we have

$$
\left\langle\tilde{G}_{\mu \nu}^{(1)}(p) \tilde{G}_{\alpha \beta}^{(1)}\left(p^{\prime}\right)\right\rangle_{\text {ind }}=\frac{\bar{\kappa}^{2}}{N}(2 \pi)^{4} \delta^{4}\left(p+p^{\prime}\right) \tilde{N}_{\mu \nu \alpha \beta}(p) .
$$

Finally, we may derive the correlations for the metric perturbations from Equations (158) or (159). In the Lorentz or harmonic gauge the linearized Einstein tensor takes the particularly simple form of Equation (155) in terms of the metric perturbation. One may derive the correlation functions for $\tilde{h}_{\mu \nu}(p)$ as it was done in Section 6.4.2 to get

$$
\left\langle\tilde{\bar{h}}_{\mu \nu}(p) \tilde{\bar{h}}_{\alpha \beta}\left(p^{\prime}\right)\right\rangle_{\text {ind }}=\frac{4}{\left(p^{2}\right)^{2}}\left\langle\tilde{G}_{\mu \nu}(p) \tilde{G}_{\alpha \beta}\left(p^{\prime}\right)\right\rangle_{\text {ind }} .
$$

There will be one possible expression for the two-point metric correlation, which corresponds to the Einstein-tensor correlation of Equation (158), and another expression corresponding to Equation (158), when the order-reduction prescription is used. We should note that, contrary to the correlation functions for the Einstein tensor, the two-point metric correlation is not gauge invariant (it is given in the Lorentz gauge). Moreover, when taking the Fourier transform to get the correlations in spacetime coordinates, there is an apparent infrared divergence when $p^{2}=0$ in the massless case. This can be seen from the expression for the noise kernel $\tilde{N}_{\mu \nu \alpha \beta}(p)$ defined in Equation (125). For the massive case no such divergence due to the factor $\theta\left(-p^{2}-4 m^{2}\right)$ exists, but as one takes the limit $m \rightarrow 0$ it will show up. This infrared divergence, however, is a gauge artifact that has been enforced by the use of the Lorentz gauge. A gauge different from the Lorentz gauge should be used in the massless case; see [203] for a more detailed discussion of this point.

Let us now write the two-point metric correlation function in spacetime coordinates for the massless and conformally coupled fields. In order to avoid runaway solutions we use the prescription that the propagator should have a well-defined Fourier transform by integrating along the real 
axis in the complex $p^{0}$ plane. This was, in fact, done in Section 6.4.3 and we may now write Equation (146) as

$$
\left\langle\tilde{\bar{h}}_{\mu \nu}(x) \tilde{\bar{h}}_{\alpha \beta}(y)\right\rangle_{\text {ind }}=\frac{\bar{\kappa}^{2}}{720 \pi N} \int \frac{d^{4} p}{(2 \pi)^{4}} \frac{e^{i p(x-y)} P_{\mu \nu \alpha \beta} \theta\left(-p^{2}\right)}{\left|1+(\bar{\kappa} / 2) p^{2} \tilde{H}\left(p ; \bar{\mu}^{2}\right)\right|^{2}},
$$

where the projector $P_{\mu \nu \alpha \beta}$ is defined in Equation (107). This correlation function for the metric perturbations is in agreement with the real part of the graviton propagator obtained by Tomboulis in [348] using a large $N$ expansion with Fermion fields. Note that when the order-reduction prescription is used the terms in the denominator of Equation (161) that are proportional to $p^{2}$ are neglected. Thus, in contrast to the intrinsic metric fluctuations, there is still a nontrivial contribution to the induced metric fluctuations due to the quantum matter fields in this case.

To estimate the above integral let us follow Section 6.4.3 and consider spacelike separated points $x-y=(0, \mathbf{r})$ and introduce the Planck length $l_{p}$. For space separations $|\mathbf{r}| \gg l_{p}$ we have that the two-point correlation (161) goes as $\sim N l_{p}^{4} /|\mathbf{r}|^{4}$, and for $|\mathbf{r}| \sim \sqrt{N} l_{p}$ we have that it goes as $\sim \exp \left(-|\mathbf{r}| / \sqrt{N} l_{p}\right) l_{p} /|\mathbf{r}|$. Since these metric fluctuations are induced by the matter stress fluctuations we infer that the effect of the matter fields is to suppress metric fluctuations at small scales. On the other hand, at large scales the induced metric fluctuations are small compared to the free graviton propagator, which goes like $l_{p}^{2} /|\mathbf{r}|^{2}$.

We thus conclude that, once the instabilities giving rise to the unphysical runaway solutions have been discarded, the fluctuations of the metric perturbations around the Minkowski spacetime induced by the interaction with quantum scalar fields are indeed stable (instabilities lead to divergent results when Fourier transforming back to spacetime coordinates). We have found that, indeed, both the intrinsic and the induced contributions to the quantum correlation functions of metric perturbations are stable, and consequently Minkowski spacetime is stable.

\subsubsection{Order-reduction prescription and large $\mathrm{N}$}

Runaway solutions are a typical feature of equations describing backreaction effects, such as in classical electrodynamics, and are due to higher than two time derivatives in the dynamical equations. Here we will give a qualitative analysis of this problem in semiclassical gravity. In a very schematic way the semiclassical Einstein equations have the form

$$
G_{h}+l_{p}^{2} \ddot{G}_{h}=0,
$$

where, say, $G_{h}$ stands for the linearized Einstein tensor over the Minkowski background and we have simplified the equation as much as possible. The second term of the equation is due to the vacuum polarization of matter fields and contains four time derivatives of the metric perturbation. Some specific examples of such an equation are, in momentum space, Equations (153) and (154). The order-reduction procedure is based on treating perturbatively the terms involving higher-order derivatives, differentiating the equation under consideration, and substituting back the higher derivative terms in the original equation, keeping only terms up to the required order in the perturbative parameter. In the case of the semiclassical Einstein equation, the perturbative parameter is $l_{p}^{2}$. If we differentiate Equation (162) with respect to time twice, it is clear that the second-order derivatives of the Einstein tensor are of order $l_{p}^{2}$. Substituting back into the original equation, we get the following equation up to order $l_{p}^{4}: G_{h}=0+O\left(l_{p}^{4}\right)$. Now there are certainly no runaway solutions but also no effect due to the vacuum polarization of matter fields. Note that the result is not so trivial when there is an inhomogeneous term on the right-hand side of Equation (162), this is what happens with the induced fluctuations predicted by the Einstein-Langevin equation.

Semiclassical gravity is expected to provide reliable results as long as the characteristic length scales under consideration, say $L$, satisfy that $L \gg l_{p}[110]$. This can be qualitatively argued by 
estimating the magnitude of the different contributions to the effective action for the gravitational field, considering the relevant Feynman diagrams and using dimensional arguments. Let us write the effective gravitational action, again in a very schematic way, as

$$
S_{\mathrm{eff}}=\int d^{4} x \sqrt{-g}\left(\frac{1}{l_{p}^{2}} R+\alpha R^{2}+l_{p}^{2} R^{3}+\ldots\right),
$$

where $R$ is the Ricci scalar. The first term is the usual classical Einstein-Hilbert term. The second stands for terms quadratic in the curvature (square of Ricci and Weyl tensors). These terms appear as radiative corrections due to vacuum polarization of matter fields. Here $\alpha$ is a dimensionless parameter presumably of order 1 and the $R^{3}$ terms are higher-order corrections, which appear, for instance, when one considers internal graviton propagators inside matter loops. Let us assume that $R \sim L^{-2}$; then the different terms in the action are on the order of $R^{2} \sim L^{-4}$ and $l_{p}^{2} R^{3} \sim l_{p}^{2} L^{-6}$. Consequently, when $L \gg l_{p}^{2}$, the term due to matter loops is a small correction to the Einstein-Hilbert term $\left(1 / l_{p}^{2}\right) R \gg R^{2}$ and this term can be treated as a perturbation. The justification for the order-reduction prescription is actually based on this fact. Therefore, significant effects from the vacuum polarization of the matter fields are only expected when their small corrections accumulate in time, as would be the case for an evaporating macroscopic black hole all the way before reaching Planckian scales (see Section 8.3).

However, if we have a large number $N$ of matter fields, the estimates for the different terms change in a remarkable way. This is interesting because the large $N$ expansion seems, as we have argued in Section 3.3.1, the best justification for semiclassical gravity. In fact, now the $N$ vacuumpolarization terms involving loops of matter are of order $N R^{2} \sim N L^{-4}$. For this reason, the contribution of the graviton loops, which is just of order $R^{2}$ as is any other loop of matter, can be neglected in front of the matter loops; this justifies the semiclassical limit. Similarly, higher-order corrections are of order $N l_{p}^{2} R^{3} \sim N l_{p}^{2} L^{-6}$. Now there is a regime, when $L \sim \sqrt{N} l_{p}$, where the Einstein-Hilbert term is comparable to the vacuum polarization of matter fields, $\left(1 / l_{p}^{2}\right) R \sim N R^{2}$, and yet the higher correction terms can be neglected because we still have $L \gg l_{p}$, provided $N \gg 1$. This is the kind of situation considered in trace anomaly driven inflationary models [162], such as that originally proposed by Starobinsky [339], see also [355], where exponential inflation is driven by a large number of massless conformal fields. The order-reduction prescription would completely discard the effect from the vacuum polarization of the matter fields even though it is comparable to the Einstein-Hilbert term. In contrast, the procedure proposed by Hawking et al. keeps the contribution from the matter fields. Note that here the actual physical Planck length $l_{p}$ is considered, not the rescaled one, $\bar{l}_{p}^{2}=\bar{\kappa} / 8 \pi$, which is related to $l_{p}$ by $l_{p}^{2}=\kappa / 8 \pi=\bar{l}_{p}^{2} / N$.

\subsubsection{Summary}

An analysis of the stability of any solution of semiclassical gravity with respect to small quantum perturbations should include not only the evolution of the expectation value of the metric perturbations around that solution, but also their fluctuations encoded in the quantum correlation functions. Making use of the equivalence (to leading order in $1 / N$, where $N$ is the number of matter fields) between the stochastic correlation functions obtained in stochastic semiclassical gravity and the quantum correlation functions for metric perturbations around a solution of semiclassical gravity, the symmetrized two-point quantum correlation function for the metric perturbations can be decomposed into two different parts: the intrinsic metric fluctuations due to the fluctuations of the initial state of the metric perturbations itself, and the fluctuations induced by their interaction with the matter fields. From the linearized perturbations of the semiclassical Einstein equation, information on the intrinsic metric fluctuations can be retrieved. On the other hand, the information on the induced metric fluctuations naturally follows from the solutions of the Einstein-Langevin equation. 
We have analyzed the symmetrized two-point quantum correlation function for the metric perturbations around the Minkowski spacetime interacting with $N$ scalar fields initially in the Minkowski vacuum state. Once the instabilities that arise in semiclassical gravity, which are commonly regarded as unphysical, have been properly dealt with by using the order-reduction prescription or the procedure proposed by Hawking et al. [161, 162], both the intrinsic and the induced contributions to the quantum correlation function for the metric perturbations are found to be stable [203]. Thus, we conclude that Minkowski spacetime is a valid solution of semiclassical gravity. 


\section{Structure Formation in the Early Universe}

Structure formation in the early universe is a key problem in modern cosmology. It is believed that galaxies, clusters and all large-scale structures observed today originated from random sources of primordial inhomogeneities (density contrast) in the early universe, amplified by the expansion of the universe. Theories of structure formation based on general relativity theory have been in existence for over 60 years [27, 241, 242] (see, e.g., [17, 284, 300]), long before the advent of inflationary cosmology [233, 245, 269]. But the inflation paradigm [2, 140, 243, 244] provided at least two major improvements in the modern theory of cosmological structure formation [19, 141, 159, 270]:

- The sources: Instead of a classical white-noise source arbitrarily specified, the seeds of structures of the new theory are from quantum fluctuations, which obey equations derivable from the dynamics of the inflaton field, which is responsible for driving inflation.

- The spectrum: The almost scale-invariant spectrum (masses of galaxies as a function of their scales) has a more natural explanation from the almost exponential expansion of the inflationary universe than from the power-law expansion of the FRW universe in the traditional theory.

Stochastic gravity provides a sound and natural formalism for the derivation of the cosmological perturbations generated during inflation. In [316] it was shown that the correlation functions that follow from the Einstein-Langevin equation, which emerges in the framework of stochastic gravity, coincide with that obtained with the usual quantization procedures [270] when both the metric perturbations and the inflaton fluctuations are linearized. Stochastic gravity, however, can naturally deal with the fluctuations of the inflaton field even beyond the linear approximation. In Section 7.4 we will enumerate possible advantages of the stochastic-gravity treatment of this problem over the usual methods based on the quantization of the linear cosmological and linear inflaton perturbations.

We should point out that the equivalence at the linearized level is proved in [316] directly from the field equations of the perturbations and by showing that the stochastic and the quantum correlations are both given by identical expressions. Within the stochastic gravity framework an explicit computation of the curvature perturbation correlations was performed by Urakawa and Maeda [353]. A convenient approximation for that computation, used by these authors, leads only to a small discrepancy with the usual approach for the observationally relevant part of the spectrum. We think the deviation from the standard result found for superhorizon modes would not arise if an exact calculation were used.

Here we illustrate the equivalence with the conventional approach with one of the simplest chaotic inflationary models in which the background spacetime is a quasi de Sitter universe [315, 316].

\subsection{The model}

In this chaotic inflationary model [245] the inflaton field $\phi$ of mass $m$ is described by the following Lagrangian density,

$$
\mathcal{L}(\phi)=\frac{1}{2} g^{a b} \nabla_{a} \phi \nabla_{b} \phi+\frac{1}{2} m^{2} \phi^{2} .
$$

The condition for the existence of an inflationary period, which is characterized by an accelerated cosmological expansion, is that the value of the field over a region with the typical size of the Hubble radius is higher than the Planck mass $m_{P}$. This is because, in order to solve the cosmological horizon and flatness problem, more than 60 e-folds of expansion are needed. To achieve this the 
scalar field should begin with a value higher than $3 m_{P}$. The inflaton mass is small; as we will see, the large scale anisotropies measured in the cosmic background radiation [334] restrict the inflaton mass to be on the order of $10^{-6} m_{P}$. We will not discuss the naturalness of this inflationary model but simply assume that if one such region is found (inside a much larger universe) it will inflate to become our observable universe.

We want to study the metric perturbations produced by the stress-energy tensor fluctuations of the inflaton field on the homogeneous background of a flat Friedmann-Robertson-Walker model, described by the cosmological scale factor $a(\eta)$, where $\eta$ is the conformal time, which is driven by the homogeneous inflaton field $\phi(\eta)=\langle\hat{\phi}\rangle$. Thus we write the inflaton field in the following form

$$
\hat{\phi}=\phi(\eta)+\hat{\varphi}(x)
$$

where $\hat{\varphi}(x)$ corresponds to a free massive quantum scalar field with zero expectation value on the homogeneous background metric: $\langle\hat{\varphi}\rangle=0$. We will restrict ourselves to scalar-type metric perturbations because these are the ones that couple to the inflaton fluctuations in the linear theory. We note that this is not so if we were to consider inflaton fluctuations beyond the linear approximation; then tensorial and vectorial metric perturbations would also arise. The perturbed metric $\tilde{g}_{a b}=g_{a b}+h_{a b}$ can be written in the longitudinal gauge as,

$$
d s^{2}=a^{2}(\eta)\left[-(1+2 \Phi(x)) d \eta^{2}+(1-2 \Psi(x)) \delta_{i j} d x^{i} d x^{j}\right]
$$

where the scalar metric perturbations $\Phi(x)$ and $\Psi(x)$ correspond to Bardeen's gauge-invariant variables [17].

\subsection{Einstein-Langevin equation for scalar metric perturbations}

The Einstein-Langevin equation as described in Section 3 is gauge invariant, and thus we can work in a desired gauge and then extract the gauge invariant quantities. The Einstein-Langevin equation (15) now reads

$$
G_{a b}^{(0)}-8 \pi G\left\langle\hat{T}_{a b}^{(0)}\right\rangle+G_{a b}^{(1)}(h)-8 \pi G\left\langle\hat{T}_{a b}^{(1)}(h)\right\rangle=8 \pi G \xi_{a b} .
$$

Note that the first two terms cancel, that is, $G_{a b}^{(0)}-8 \pi G\left\langle\hat{T}_{a b}^{(0)}\right\rangle=0$, if the background metric is a solution of the semiclassical Einstein equations. Here the superscripts (0) and (1) refer to functions in the background metric $g_{a b}$ and functions, which are linear in the metric perturbation $h_{a b}$, respectively. The stress tensor operator $\hat{T}_{a b}$ for the minimally-coupled inflaton field in the perturbed metric is

$$
\hat{T}_{a b}=\tilde{\nabla}_{a} \hat{\phi} \tilde{\nabla}_{b} \hat{\phi}+\frac{1}{2} \tilde{g}_{a b}\left(\tilde{\nabla}_{c} \hat{\phi} \tilde{\nabla}^{c} \hat{\phi}+m^{2} \hat{\phi}^{2}\right) .
$$

Using the decomposition of the scalar field into its homogeneous and inhomogeneous parts, see Equation (165), and of the metric $\tilde{g}_{a b}$ into its homogeneous background $g_{a b}$ and its perturbation $h_{a b}$, the renormalized expectation value for the stress-energy tensor operator can be written as

$$
\left\langle\hat{T}_{a b}^{R}[\tilde{g}]\right\rangle=\left\langle\hat{T}_{a b}[\tilde{g}]\right\rangle_{\phi \phi}+\left\langle\hat{T}_{a b}[\tilde{g}]\right\rangle_{\phi \varphi}+\left\langle\hat{T}_{a b}^{R}[\tilde{g}]\right\rangle_{\varphi \varphi}
$$

where the subindices indicate the degree of dependence on the homogeneous field $\phi$ and its perturbation $\varphi$. The first term in this equation depends only on the homogeneous field and it is given by the classical expression. The second term is proportional to $\langle\hat{\varphi}[\tilde{g}]\rangle$, which is not zero because the field dynamics are considered on the perturbed spacetime, i.e., this term includes the coupling of the field with $h_{a b}$ and may be obtained from the expectation value of the linearized Klein-Gordon equation,

$$
\left(\square_{g+h}-m^{2}\right) \hat{\varphi}=0 \text {. }
$$


The last term in Equation (169) corresponds to the expectation value of the stress tensor for a free scalar field on the spacetime of the perturbed metric.

After using the previous decomposition, the noise kernel $N_{a b c d}[g ; x, y)$ defined in Equation (12) can be written as

$$
\left\langle\left\{\hat{t}_{a b}[g ; x), \hat{t}_{c d}[g ; y)\right\}\right\rangle=\left\langle\left\{\hat{t}_{a b}[g ; x), \hat{t}_{c d}[g ; y)\right\}\right\rangle_{(\phi \varphi)^{2}}+\left\langle\left\{\hat{t}_{a b}[g ; x), \hat{t}_{c d}[g ; y)\right\}\right\rangle_{(\varphi \varphi)^{2}},
$$

where we have used the fact that $\langle\hat{\varphi}\rangle=0=\langle\hat{\varphi} \hat{\varphi} \hat{\varphi}\rangle$ for Gaussian states on the background geometry. We consider the vacuum state to be the Euclidean vacuum, which is Gaussian and is the preferred state in the de Sitter background. In the above equation the first term is quadratic in $\hat{\varphi}$, whereas the second one is quartic. Both contributions to the noise kernel are separately conserved, since both $\phi(\eta)$ and $\hat{\varphi}$ satisfy the Klein-Gordon field equations on the background spacetime. Consequently, the two terms can be considered separately. On the other hand, if one treats $\hat{\varphi}$ as a small perturbation the second term in (171) is of lower order than the first and may be consistently neglected. This corresponds to neglecting the last term of Equation (169). The stress tensor fluctuations due to a term of that kind were considered in [314].

We can now write down the Einstein-Langevin equations (167) to linear order in the inflaton fluctuations. It is easy to check [316] that the space-space components coming from the stress-tensor expectation-value terms and the stochastic tensor are diagonal, i.e., $\left\langle\hat{T}_{i j}\right\rangle=0=\xi_{i j}$ for $i \neq j$. This, in turn, implies that the two functions characterizing the scalar metric perturbations are equal: $\Phi=\Psi$ in agreement with [270]. The equation for $\Phi$ can be obtained from the $0 i$-component of the Einstein-Langevin equation, which, neglecting a nonlocal term, reads in Fourier space as,

$$
2 i k_{i}\left(\mathcal{H} \Phi_{k}+\Phi_{k}^{\prime}\right)=8 \pi G\left(\xi_{0 i}\right)_{k},
$$

where $k_{i}$ is the comoving momentum component associated to the comoving coordinate $x^{i}$, and we have used the definition $\Phi_{k}(\eta)=\int d^{3} x \exp (-i \vec{k} \cdot \vec{x}) \Phi(\eta, \vec{x})$. Here primes denote derivatives with respect to the conformal time $\eta$ and $\mathcal{H}=a^{\prime} / a$. A nonlocal term of dissipative character, which comes from the second term in Equation (169), should also appear on the left-hand side of Equation (172), but we have neglected it to simplify the forthcoming expressions (the large scale spectrum does not change in a substantial way). We must emphasize, however, that the proof of the equivalence of the stochastic approach to linear order in $\hat{\varphi}$ to the usual linear cosmological perturbations approach does not assume that simplification [316]. To solve Equation (172), whose left-hand side comes from the linearized Einstein tensor for the perturbed metric [270], we need the retarded propagator for the gravitational potential $\Phi_{k}$,

$$
G_{k}\left(\eta, \eta^{\prime}\right)=-i \frac{4 \pi}{k_{i} m_{P}^{2}}\left(\theta\left(\eta-\eta^{\prime}\right) \frac{a\left(\eta^{\prime}\right)}{a(\eta)}+f\left(\eta, \eta^{\prime}\right)\right),
$$

where $f$ is a homogeneous solution of Equation (172) related to the initial conditions chosen and $m_{P}^{2}=1 / G$. For instance, if we take $f\left(\eta, \eta^{\prime}\right)=-\theta\left(\eta_{0}-\eta^{\prime}\right) a\left(\eta^{\prime}\right) / a(\eta)$, the solution would correspond to "turning on" the stochastic source at $\eta_{0}$. With the solution of the Einstein-Langevin equation (172) for the scalar metric perturbations we are in position to compute the two-point correlation functions for these perturbations.

\subsection{Correlation functions for scalar metric perturbations}

The two-point correlation function for the scalar metric perturbations induced by the inflaton fluctuations is thus given by

$$
\begin{aligned}
& \left\langle\Phi_{k}(\eta) \Phi_{k^{\prime}}\left(\eta^{\prime}\right)\right\rangle_{s}=(2 \pi)^{2} \delta\left(\vec{k}+\vec{k}^{\prime}\right) \\
& \times \int^{\eta} d \eta_{1} \int^{\eta^{\prime}} d \eta_{2} G_{k}\left(\eta, \eta_{1}\right) G_{k^{\prime}}\left(\eta^{\prime}, \eta_{2}\right)\left\langle\left(\xi_{0 i}\right)_{k}\left(\eta_{1}\right)\left(\xi_{0 i}\right)_{k^{\prime}}\left(\eta_{2}\right)\right\rangle_{s} .
\end{aligned}
$$


Here the two-point correlation function for the stochastic source, which is connected to the stressenergy tensor fluctuations through the noise kernel, is given by,

$$
\begin{aligned}
\left\langle\left(\xi_{0 i}\right)_{k}\left(\eta_{1}\right)\left(\xi_{0 i}\right)_{-k}\left(\eta_{2}\right)\right\rangle_{s} & =\frac{1}{2}\left\langle\left\{\left(\hat{t}_{0 i}\right)_{k}\left(\eta_{1}\right),\left(\hat{t}_{0 i}\right)_{-k}\left(\eta_{2}\right)\right\}\right\rangle_{\phi \varphi} \\
& =\frac{1}{2} k_{i} k_{i} \phi^{\prime}\left(\eta_{1}\right) \phi^{\prime}\left(\eta_{2}\right) G_{k}^{(1)}\left(\eta_{1}, \eta_{2}\right)
\end{aligned}
$$

where $G_{k}^{(1)}\left(\eta_{1}, \eta_{2}\right)=\left\langle\left\{\hat{\varphi}_{k}\left(\eta_{1}\right), \hat{\varphi}_{-k}\left(\eta_{2}\right)\right\}\right\rangle$ is the $k$ th-mode Hadamard function for a free minimally coupled scalar field in the appropriate vacuum state on the Friedmann-Robertson-Walker background.

In practice, to make the explicit computation of the Hadamard function, we will assume that the field state is in the Euclidean vacuum and the background spacetime is de Sitter. Furthermore, we will compute the Hadamard function for a massless field, and will make a perturbative expansion in terms of the dimensionless parameter $m / m_{P}$. Thus we consider

$$
\bar{G}_{k}^{(1)}\left(\eta_{1}, \eta_{2}\right)=\left\langle 0\left|\left\{\hat{y}_{k}\left(\eta_{1}\right), \hat{y}_{-k}\left(\eta_{2}\right)\right\}\right| 0\right\rangle=2 \mathcal{R}\left(u_{k}\left(\eta_{1}\right) u_{k}^{*}\left(\eta_{2}\right)\right)
$$

with

and where

$$
\hat{y}_{k}(\eta)=a(\eta) \hat{\varphi}_{k}(\eta)=\hat{a}_{k} u_{k}(\eta)+\hat{a}_{-k}^{\dagger} u_{-k}^{*}(\eta)
$$

$$
u_{k}=(2 k)^{-1 / 2} e^{i k \eta}(1-i / \eta)
$$

are the positive frequency $k$-modes for a massless minimally-coupled scalar field on a de Sitter background, which define the Euclidean vacuum state $\hat{a}_{k}|0\rangle=0$ [34].

The assumption of a massless field for the computation of the Hadamard function is made because massless modes in de Sitter are much simpler to deal with than massive modes. We can see that this is nonetheless a reasonable approximation as follows: For a given mode, the $m=0$ approximation is reasonable when its wavelength $\lambda$ is shorter than the Compton wavelength, $\lambda_{c}=1 / m$. In our case we have a very small mass $m$ and the horizon size $H^{-1}$, where $H$ is the Hubble constant $H=\dot{a} / a$ (here $a(t)$ with $t$ the physical time $d t=a d \eta$ ), satisfies $H^{-1}<\lambda_{c}$. Thus, for modes inside the horizon, $\lambda<\lambda_{c}$ and $m=0$ are a good approximation. Outside the horizon, massive modes decay in amplitude as $\sim \exp \left(-m^{2} t / 3 H\right)$ whereas massless modes remain constant. Thus, when modes leave the horizon, the approximation will eventually break down. However, we only need to ensure that the approximation is still valid after 60 e-folds, i.e., $H \Delta t \sim 60(\Delta t$ being the time between horizon exit and the end of inflation). But this is the case provided $3 H^{2} / \mathrm{m}^{2}>60$, since the decay factor $\sim \exp \left[-\left(m^{2} / 3 H^{2}\right) H \Delta t\right]$ will not be too different from unity for those modes that left the horizon during the last sixty e-folds of inflation. This condition is indeed satisfied given that $m \ll H$ in most slow-roll inflationary models [233, 284] and in particular for the model considered here, in which $m \sim 10^{-6} m_{P}$.

We note that the background geometry is not exactly that of de Sitter spacetime, for which $a(\eta)=-(H \eta)^{-1}$ with $-\infty<\eta<0$. One can expand in terms of the "slow-roll" parameters and assume that, to first order, $\dot{\phi}(t) \simeq m_{P}^{2}\left(\mathrm{~m} / m_{P}\right)$, where $t$ is the physical time. The correlation function for the metric perturbation (175) can then be easily computed; see [315, 316] for details. The final result, however, is very weakly dependent on the initial conditions as one may understand from the fact that the accelerated expansion of the quasi-de Sitter spacetime during inflation erases the information about the initial conditions. Thus, one may take the initial time to be $\eta_{0}=-\infty$ and obtain to lowest order in $m / m_{P}$ the expression

$$
\left\langle\Phi_{k}(\eta) \Phi_{k^{\prime}}\left(\eta^{\prime}\right)\right\rangle_{s} \simeq 8 \pi^{2}\left(\frac{m}{m_{P}}\right)^{2} k^{-3}(2 \pi)^{3} \delta\left(\vec{k}+\vec{k}^{\prime}\right) \cos k\left(\eta-\eta^{\prime}\right) .
$$


From this result two main conclusions can be derived. First, the prediction of an almost scaleinvariant Harrison-Zel'dovich spectrum for large scales, i.e., small values of $k$. Second, since the correlation function is on the order of $\left(m / m_{P}\right)^{2}$, a severe bound to the mass $m$ is imposed by the gravitational fluctuations derived from the small values of the cosmic microwave background (CMB) anisotropies detected by COBE. This bound is on the order of $\left(m / m_{P}\right) \sim 10^{-6}[270,334]$.

We now comment on some differences with [53, 60, 262, 263], which used a self-interacting scalar field or a scalar field interacting nonlinearly with other fields. In these works an important relaxation of the ratio $m / m_{P}$ was found. The long wavelength modes of the inflaton field were regarded as an open system in an environment made out of the shorter wavelength modes. Then, Langevin type equations were used to compute the correlations of the long wavelength modes driven by the fluctuations of the shorter wavelength modes. In order to get a significant relaxation on the above ratio, however, one had to assume that the correlations of the free long-wavelength modes, which correspond to the dispersion of the system's initial state, were very small. Otherwise they dominate by several orders of magnitude those fluctuations that come from the noise of the environment. This would require a great amount of fine-tuning for the initial quantum state of each mode [316].

We should remark that in the linear model discussed here there is no environment for the inflaton fluctuations. When one linearizes with respect to both the scalar metric perturbations and the inflaton perturbations, the system cannot be regarded as a true open quantum system. The reason is that Fourier modes decouple and the dynamical constraints due to diffeomorphism invariance link the metric perturbations of scalar type with the perturbations of the inflaton field so that only one true dynamical degree of freedom is left for each Fourier mode. Nevertheless, the inflaton fluctuations are responsible for the noise that induces the metric perturbations.

\subsection{Summary and outlook}

Stochastic gravity provides an alternative framework to study the generation of primordial inhomogeneities in inflationary models. Besides the interest of the problem in its own right, there are also other reasons that make this problem worth discussing from the point of view of stochastic gravity. The Einstein-Langevin equation is not restricted by the use of linearized perturbations of the inflaton field. In practice this may not be very important for inflationary models, which are driven by an inflaton field, which takes a non-zero expectation value, because the linear perturbations will give the leading contribution. However, the importance of considering corrections due to one-loop contributions from the inflaton-field perturbations, beyond the tree level of the linear cosmological perturbation theory, has recently been emphasized by Weinberg [368, 369] as a means to understand the consequences of the theory even where in practice its predictions may not be observed. Note also $[225,226]$ for the effect of the quantum gravitational loop corrections on the dynamics of the inflaton field.

In the stochastic gravity approach some insights on the exact treatment of the inflaton scalar field perturbations have been discussed in [316, 353, 354]. The main features that would characterize an exact treatment of the inflaton perturbations are the following. First, the three types of metric perturbations (scalar, vectorial and tensorial perturbations) couple to the perturbations of the inflaton field. Second, the corresponding Einstein-Langevin equation for the linear metric perturbations will explicitly couple to the scalar and tensorial metric perturbations. Furthermore, although the Fourier modes (with respect to the spatial coordinates) for the metric perturbations will still decouple in the Einstein-Langevin equation, any given mode of the noise and dissipation kernels will get contributions from an infinite number of Fourier modes of the inflaton field perturbations. This fact will imply, in addition, the need to properly renormalize the ultraviolet divergences arising in the dissipation kernel, which actually correspond to the divergences associated with the expectation value of the stress-tensor operator of the quantum matter field evolving 
on the perturbed geometry.

We should remark that although the gravitational fluctuations are here assumed to be classical, the correlation functions obtained correspond to the expectation values of the symmetrized quantum metric perturbations $[66,316]$. This means that even in the absence of decoherence, the fluctuations predicted by the Einstein-Langevin equation still give the correct symmetrized quantum two-point correlation functions. In [66] it was explained how a stochastic description based on a Langevin-type equation could be introduced to gain information on fully quantum properties of simple linear open systems. In a forthcoming paper [317] it will be shown that, by carefully dealing with the gauge freedom and the consequent dynamical constraints, this result can be extended to the case of $N$ free quantum matter fields interacting with the metric perturbations around a given background. In particular, the correlation functions for the metric perturbations obtained using the Einstein-Langevin equation are equivalent to the correlation functions that would follow from a purely quantum field theory calculation up to the leading order contribution in the large $N$ limit. This will generalize the results already obtained on a Minkowski background [203, 204].

These results have important implications on the use of the Einstein-Langevin equation to address situations in which the background configuration for the scalar field vanishes. This includes not only the case of a Minkowski background spacetime, but also the remarkably interesting case of the trace anomaly-induced inflation. That is, inflationary models driven by the vacuum polarization of a large number of conformal fields [162, 339, 355], in which the usual approaches based on the linearization of both the metric perturbations and the scalar field perturbations and their subsequent quantization can no longer be applied. More specifically, the semiclassical Einstein equations (8) for massless quantum fields conformally coupled to the gravitational field admit an inflationary solution that begins in an almost de Sitter-like regime and ends up in a matter-dominated-like regime [339, 355]. In these models the standard approach based on the quantization of the gravitational and the matter fields to linear order cannot be used because the calculation of the metric perturbations correspond to having only the last term in the noise kernel in Equation (171), since there is no homogeneous field $\phi(\eta)$ as the expectation value $\langle\hat{\phi}\rangle=0$ and linearization becomes trivial.

In the trace anomaly induced inflation model Hawking et al. [162] were able to compute the two-point quantum correlation function for scalar and tensorial metric perturbations in a spatiallyclosed de Sitter universe, making use of the anti-de Sitter/conformal field theory correspondence. They find that short-scale metric perturbations are strongly suppressed by the conformal matter fields. This is similar to what we obtained in Section 6 for the induced metric fluctuations in Minkowski spacetime. In the stochastic gravity context, the noise kernel in a spatially-closed de Sitter background was derived in [314], and in a spatially-flat arbitrary Friedmann-RobertsonWalker model the Einstein-Langevin equations describing the metric perturbations were first obtained in [73]. The computation of the corresponding two-point correlation functions for the metric perturbations is now in progress. 


\section{Black Hole Backreaction and Fluctuations}

As another illustration of the application of stochastic gravity we now consider the backreaction and fluctuations in black hole spacetimes. Backreaction refers to the quantum effects of matter fields such as vacuum polarization, quantum fluctuations and particle creation on the spacetime structure and dynamics. Studying the dynamics of quantum fields in a fixed background spacetime, Hawking found that black holes emit thermal radiation with a temperature inversely proportional to their mass $[156,213,294,358]$. When the backreaction of the quantum fields on the spacetime dynamics is included, one expects that the mass of the black hole decreases as thermal radiation at higher and higher temperatures is emitted. The reduction of the mass of a black hole due to particle creation is often referred to as the black hole 'evaporation' process. Backreaction of Hawking radiation $[9,18,142,166,167,380,381,382]$ could alter the evolution of the background spacetime and change the nature of its end state, more drastically so for Planck-size black holes.

Backreaction is a technically challenging but conceptually rewarding problem. Progress is slow in this long standing problem, but it cannot be ignored because existing results from test-field approximations or semiclassical analysis are not trustworthy when backreaction becomes strong enough as to alter the structure and dynamics of the background spacetime. At the least one needs to know how strong the backreaction effects are, and under what circumstances the existing predictions make sense. Without an exact quantum solution of the black-hole-plus-quantum-field system or at least a full backreaction consideration including the intrinsic and induced effects of metric fluctuations, much of the long speculation on the end-state of black hole collapse remnants, naked singularity, baby universe formation or complete evaporation (see, e.g., [12, 47, $130,168,184,250,306,318,319,375,376]$ ) - and the information loss issue [157, 158, 160, 286] (see, e.g., $[288,307]$ for an overview and recent results from quantum information $[40,333]$ ) will remain speculation and puzzles. This issue also enters into the extension of the well-known black-hole thermodynamics $[23,25,26,171,172,214,224,253,254,337,342,346,363,364]$ to nonequilibrium conditions [105] and can lead to new inferences on the microscopic structure of spacetime and the true nature of Einstein's equations [216] from the viewpoint of general relativity as geometro-hydrodynamics and gravity as emergent phenomena. (See the non-traditional views of Volovik [356, 357] and $\mathrm{Hu}[185,186,190]$ on spacetime structure, Wen [139, 240, 372, 373] on quantum order, Seiberg [326], Horowitz and Polchinsky [173] on emergent gravity, Herzog on the hydrodynamics of M-theory [164] and the seminal work of Unruh and Jacobson [215, 351] leading to analog gravity $[15,16,320]$.

\subsection{General issues of backreaction}

Backreaction studies of quantum field-processes in cosmological spacetimes have progressed further than the corresponding black-hole problems, partly because of the relative technical simplicity associated with the higher symmetry of relevant cosmological-background geometries. (For a summary of the cosmological backreaction problem treated in stochastic-gravity theory, see [208].) How the problem is set up and approached, e.g., via effective action, in these previously-studied models can be carried over to black-hole problems. In fact, since the interior of a black hole can be described by a cosmological model (e.g., the Kantowski-Sachs universe for a spherically symmetric black hole), some aspects even convey directly. The latest important work on this problem is that of Hiscock, Larson and Anderson [165] on backreaction in the interior of a black hole, in which one can find a concise summary of earlier work.

\subsubsection{Regularized energy-momentum tensor}

The first step in a backreaction problem is to find a regularized energy-momentum tensor of the quantum fields using reasonable techniques, since the expectation value of this serves as the 
source in the semiclassical Einstein equation. For this, much work started in the 1980s (and still ongoing sparingly) is concerned with finding the right approximations for the regularized energy-momentum tensor $[6,7,8,165,217,260,292]$. Even in the simplest spherically symmetric spacetime, including the important Schwarzschild metric, it is technically quite involved. To name a few of the important landmarks in this endeavor (this is adopted from [165]), Howard and Candelas $[177,178]$ have computed the stress-energy of a conformally-invariant scalar field in the Schwarzschild geometry; Jensen and Ottewill [218] have computed the vacuum stress-energy of a massless vector field in Schwarzschild. Approximation methods have been developed by Page, Brown, and Ottewill [41, 42, 287] for conformally-invariant fields in Schwarzschild spacetime, by Frolov and Zel'nikov [120] for conformally-invariant fields in a general static spacetime, and by Anderson, Hiscock and Samuel [7, 8] for massless arbitrarily-coupled scalar fields in a general static spherically-symmetric spacetime. Furthermore, the DeWitt-Schwinger approximation has been derived by Frolov and Zel'nikov $[118,119]$ for massive fields in Kerr spacetime, and by Anderson, Hiscock and Samuel $[7,8]$ for a general (arbitrary curvature coupling and mass) scalar field in a general, static, spherically-symmetric spacetime. And they have applied their method to the Reissner-Nordström geometry [6]. Though arduous and demanding, the effort continues on because of its importance in finding the backreaction effects of Hawking radiation on the evolution of black holes and the quantum structure of spacetime.

Here we wish to address the black hole backreaction problem with new insights and methods provided by stochastic gravity. (For the latest developments, see, e.g., [183, 187, 207, 208].) It is not our intention to seek better approximations for the regularized energy-momentum tensor, but to point out new ingredients lacking in the existing semiclassical-gravity framework. In particular one needs to consider both the dissipation and the fluctuation aspects in the backreaction of particle creation and vacuum polarization.

In a short note $\mathrm{Hu}$, Raval and Sinha [199] first used the stochastic gravity formalism to address the backreaction of evaporating black holes. A more detailed analysis is given by the recent work of $\mathrm{Hu}$ and Roura [201, 202]. For the class of quasi-static black holes, the formulation of the problem in this new light was sketched out by Sinha, Raval, and $\mathrm{Hu}$ [332]. We follow these two latter works in the stochastic gravity theory approach to the black-hole fluctuations and backreaction problems.

\subsubsection{Backreaction and fluctuation-dissipation relation}

From the statistical field-theory perspective provided by stochastic gravity, one can understand that backreaction effect is the manifestation of a fluctuation-dissipation relation $[48,49,103,104$, 274, 365]. This was first conjectured by Candelas and Sciama [76, 324, 325] for a dynamic Kerr black hole emitting Hawking radiation and Mottola [268] for a static black hole (in a box) in quasiequilibrium with its radiation via linear-response theory [33, 234, 235, 236, 238]. This postulate was shown to hold for fully dynamical spacetimes. From the cosmological-backreaction problem $\mathrm{Hu}$ and Sinha [206] derived a generalized fluctuation-dissipation relation relating dissipation (of anisotropy in Bianchi Type I universes) and fluctuations (measured by particle numbers created in neighboring histories).

While the fluctuation-dissipation relation in linear-response theory captures the response of the system (e.g., dissipation of the black hole) to the environment (in these cases the quantum matter field), linear-response theory (in the way it is commonly presented in statistical thermodynamics) cannot provide a full description of self-consistent backreaction on at least two counts:

First, because it is usually based on the assumption of a specified background spacetime (static in this case) and state (thermal) of the matter field(s) (e.g., [268]). The spacetime and the state of matter should be determined in a self-consistent manner by their dynamics and mutual influence. Second, the fluctuation part represented by the noise kernel is amiss, e.g., [10, 11]. This is also a problem in the fluctuation-dissipation relation proposed by Candelas and Sciama [76, 324, 325] 
(see below). As demonstrated by many authors [73, 206] backreaction is intrinsically a dynamic process. The Einstein-Langevin equation in stochastic gravity overcomes both of these deficiencies.

For Candelas and Sciama [76, 324, 325], the classical formula they showed relating the dissipation in area linearly to the squared absolute value of the shear amplitude is suggestive of a fluctuation-dissipation relation. When the gravitational perturbations are quantized (they choose the quantum state to be the Unruh vacuum) they argue that it approximates a flux of radiation from the hole at large radii. Thus the dissipation in area due to the Hawking flux of gravitational radiation is allegedly related to the quantum fluctuations of gravitons. The criticism in [199] is that their's is not a fluctuation-dissipation relation in the truly statistical mechanical sense because it does not relate dissipation of a certain quantity (in this case, horizon area) to the fluctuations of the same quantity. To do so would require one to compute the two-point function of the area, which, being a four-point function of the graviton field, is related to a two-point function of the stress tensor. The stress tensor is the true "generalized force" acting on the spacetime via the equations of motion, and the dissipation in the metric must eventually be related to the fluctuations of this generalized force for the relation to qualify as a fluctuation-dissipation relation.

\subsubsection{Noise and fluctuations - the missing ingredient in older treatments}

From this reasoning, we see that the vacuum expectation value of the stress-energy bitensor, known as the noise kernel, is the necessary new ingredient in addition to the dissipation kernel, and that stochastic gravity as an extension of semiclassical gravity is the appropriate framework for backreaction considerations. The noise kernel for quantum fields in Minkowski and de Sitter spacetime has been carried out by Martin, Roura and Verdaguer [257, 259, 316], and for thermal fields in black-hole spacetimes and scalar fields in general spacetimes by Campos, $\mathrm{Hu}$ and Phillips [69, 70, 304, 305].

\subsection{Backreaction on black holes under quasi-static conditions}

As an illustration of the application of stochastic-gravity theory we outline the essential steps in a black-hole backreaction calculation, focusing on a more manageable quasi-static class. We adopt the Hartle-Hawking picture [151] where the black hole is bathed eternally - actually in quasithermal equilibrium - in the Hawking radiance it emits. It is described here by a massless scalar quantum field at the Hawking temperature. As is well known, this quasi-equilibrium condition is possible only if the black hole is enclosed in a box of size suitably larger than the event horizon. We can divide our consideration into the far-field case and the near-horizon case. Campos and $\mathrm{Hu}[69,70]$ have treated a relativistic thermal plasma in a weak gravitational field. Since the farfield limit of a Schwarzschild metric is just the perturbed Minkowski spacetime, one can perform a perturbation expansion of hot flat space using the thermal Green's functions [129]. Strictly speaking, the location of the box holding the black hole in equilibrium with its thermal radiation is as far as one can go, thus the metric may not reach the perturbed Minkowski form. But one can also put the black hole and its radiation in an anti-de Sitter space [163], which contains such a region. Hot flat space has been studied before for various purposes. See, e.g., [39, 89, 138, 311, 312]. Campos and Hu derived a stochastic CTP effective action and from it an equation of motion, the Einstein Langevin equation, for the dynamical effect of a scalar quantum field on a background spacetime. To perform calculations leading to the Einstein-Langevin equation one needs to begin with a self-consistent solution of the semiclassical Einstein equation for the thermal field and the perturbed background spacetime. For a black-hole background, a semiclassical gravity solution is provided by York [380, 381, 382]. For a Robertson-Walker background with thermal fields it is given by $\mathrm{Hu}[180]$.

We follow the strategy outlined by Sinha, Raval and Hu [332] for treating the near horizon case, following the same scheme of Campos and Hu. In both cases two new terms appear, which are 
absent in semiclassical gravity considerations: a nonlocal dissipation and a (generally colored) noise kernel. When one takes the noise average one recovers York's [380, 381, 382] semiclassical equations for radially perturbed quasi-static black holes. For the near-horizon case one cannot obtain the full details yet, because the Green's function for a scalar field in the Schwarzschild metric comes only in an approximate form, e.g., Page's approximation [287], which, though reasonably accurate for the stress tensor, fails badly for the noise kernel [305]. In addition, a formula is derived in [332] expressing the CTP effective action in terms of the Bogoliubov coefficients. Since it measures not only the number of particles created, but also the difference of particle creation in alternative histories, this provides a useful avenue to explore the wider set of issues in black hole physics related to noise and fluctuations.

Since backreaction calculations in semiclassical gravity have been under study for a much longer time than in stochastic gravity we will concentrate on explaining how the new stochastic features arise from the framework of semiclassical gravity, i.e., noise and fluctuations and their consequences. Technically the goal is to obtain an influence action for this model of a black hole coupled to a scalar field and to derive an Einstein-Langevin equation from it. As a by-product, from the fluctuation-dissipation relation, one can derive the vacuum-susceptibility function and the isothermal-compressibility function for black holes, two quantities of fundamental interest in characterizing the nonequilibrium thermodynamic properties of black holes.

\subsubsection{The model}

In this model the black-hole spacetime is described by a spherically-symmetric static metric with line element of the following general form written in advanced-time Eddington-Finkelstein coordinates as

$$
d s^{2}=g_{\mu \nu} d x^{\mu} d x^{\nu}=-e^{2 \psi}\left(1-\frac{2 m}{r}\right) d v^{2}+2 e^{2 \psi} d v d r+r^{2} d \Omega^{2},
$$

where $\psi=\psi(r), m=m(r), v=t+r+2 M \ln \left(\frac{r}{2 M}-1\right)$, and $d \Omega^{2}$ is the line element on the two-sphere. Hawking radiation is described by a massless, conformally-coupled quantum scalar field $\phi$ with the classical action

$$
S_{\mathrm{m}}\left[\phi, g_{\mu \nu}\right]=-\frac{1}{2} \int d^{n} x \sqrt{-g}\left[g^{\mu \nu} \partial_{\mu} \phi \partial_{\nu} \phi+\xi(n) R \phi^{2}\right]
$$

where $\xi(n)=\frac{(n-2)}{4(n-1)}$ ( $n$ is the dimension of spacetime) and $R$ is the curvature scalar of the spacetime it lives in.

Let us consider linear perturbations $h_{\mu \nu}$ of a background Schwarzschild metric $g_{\mu \nu}^{(0)}$,

$$
g_{\mu \nu}=g_{\mu \nu}^{(0)}+h_{\mu \nu}
$$

with standard line element

$$
\left(d s^{2}\right)^{0}=\left(1-\frac{2 M}{r}\right) d v^{2}+2 d v d r+r^{2} d \Omega^{2} .
$$

We look for this class of perturbed metrics in the form given by Equation (179) (thus restricting our consideration only to spherically symmetric perturbations),

$$
e^{\psi} \simeq 1+\epsilon \rho(r)
$$

and

$$
m \simeq M[1+\epsilon \mu(r)]
$$


where $\epsilon /\left(\lambda M^{2}\right)=\frac{1}{3} a T_{\mathrm{H}}^{4}$ with $a=\frac{\pi^{2}}{30}$ and $\lambda=90\left(8^{4}\right) \pi^{2}$, and where $T_{\mathrm{H}}$ is the Hawking temperature. This particular parameterization of the perturbation is chosen following York's notation [380, 381, 382]. Thus, the only non-zero components of $h_{\mu \nu}$ are

$$
h_{v v}=-\left[\left(1-\frac{2 M}{r}\right) 2 \epsilon \rho(r)+\frac{2 M \epsilon \mu(r)}{r}\right],
$$

and

$$
h_{v r}=\epsilon \rho(r) \text {. }
$$

So this represents a metric with small static and radial perturbations about a Schwarzschild black hole. The initial quantum state of the scalar field is taken to be the Hartle-Hawking vacuum, which is essentially a thermal state at the Hawking temperature, and it represents a black hole in (unstable) thermal equilibrium with its own Hawking radiation. In the far-field limit, the gravitational field is described by a linear perturbation of Minkowski spacetime. In equilibrium the thermal bath can be characterized by a relativistic fluid with a four-velocity (time-like normalized vector field) $u^{\mu}$ and temperature in its own rest frame $\beta^{-1}$.

To facilitate later comparisons with our program we briefly recall York's work [380, 381, 382]. (See also the work by Hochberg and Kephart [166] for a massless vector field, by Hochberg, Kephart, and York [167] for a massless spinor field, and by Anderson, Hiscock, Whitesell, and York [9] for a quantized massless scalar field with arbitrary coupling to spacetime curvature.) York considered the semiclassical Einstein equation,

$$
G_{\mu \nu}\left(g_{\alpha \beta}\right)=\kappa\left\langle T_{\mu \nu}\right\rangle,
$$

with $G_{\mu \nu} \simeq G_{\mu \nu}^{(0)}+\delta G_{\mu \nu}$, where $G_{\mu \nu}^{(0)}$ is the Einstein tensor for the background spacetime. The zeroth-order solution gives a background metric in empty space, i.e, the Schwarzschild metric. $\delta G_{\mu \nu}$ is the linear correction to the Einstein tensor in the perturbed metric. The semiclassical Einstein equation in this approximation therefore reduces to

$$
\delta G_{\mu \nu}\left(g^{(0)}, h\right)=\kappa\left\langle T_{\mu \nu}\right\rangle .
$$

York solved this equation to first order by using the expectation value of the energy-momentum tensor for a conformally-coupled scalar field in the Hartle-Hawking vacuum in the unperturbed (Schwarzschild) spacetime on the right-hand side and using Equations (185) and (186) to calculate $\delta G_{\mu \nu}$ on the left-hand side. Unfortunately, no exact analytical expression is available for the $\left\langle T_{\mu \nu}\right\rangle$ in a Schwarzschild metric with the quantum field in the Hartle-Hawking vacuum that goes on the right-hand side. York, therefore, used the approximate expression given by Page [287], which is known to give excellent agreement with numerical results. Page's approximate expression for $\left\langle T_{\mu \nu}\right\rangle$ was constructed using a thermal Feynman Green's function obtained by a conformal transformation of a WKB-approximated Green's function for an optical Schwarzschild metric. York then solves the semiclassical Einstein equation (188) self-consistently to obtain the corrections to the background metric induced by the backreaction encoded in the functions $\mu(r)$ and $\rho(r)$. There was no mention of fluctuations or its effects. As we shall see, in the language of Section 4, the semiclassical gravity procedure, which York followed, working at the equation of motion level, is equivalent to looking at the noise-averaged backreaction effects.

\subsubsection{CTP effective action for the black hole}

We first derive the CTP effective action for the model described in the previous section. Using the metric (182) (and neglecting the surface terms that appear in an integration by parts) we have the action for the scalar field written perturbatively as

$$
S_{\mathrm{m}}\left[\phi, h_{\mu \nu}\right]=\frac{1}{2} \int d^{n} x \sqrt{-g^{(0)}} \phi\left[\square^{(0)}+V^{(1)}+V^{(2)}+\ldots\right] \phi,
$$


where the first and second order perturbative operators $V^{(1)}$ and $V^{(2)}$ are given by

$$
\begin{aligned}
& V^{(1)} \equiv-\frac{1}{\sqrt{-g^{(0)}}}\left\{\partial_{\mu}\left(\sqrt{-g^{(0)}} \bar{h}^{\mu \nu}\right) \partial_{\nu}+\bar{h}^{\mu \nu} \partial_{\mu} \partial_{\nu}+\xi(n) R^{(1)}\right\}, \\
& V^{(2)} \equiv-\frac{1}{\sqrt{-g^{(0)}}}\left\{\partial_{\mu}\left(\sqrt{-g^{(0)}} \hat{h}^{\mu \nu}\right) \partial_{\nu}+\hat{h}^{\mu \nu} \partial_{\mu} \partial_{\nu}-\xi(n)\left(R^{(2)}+\frac{1}{2} h R^{(1)}\right)\right\} .
\end{aligned}
$$

In the above expressions, $R^{(k)}$ is the $k$-order term in the perturbation $h_{\mu \nu}(x)$ of the scalar curvature $R$ and $\bar{h}_{\mu \nu}$ and $\hat{h}_{\mu \nu}$ denote a linear and a quadratic combination of the perturbation, respectively:

$$
\begin{aligned}
& \bar{h}_{\mu \nu} \equiv h_{\mu \nu}-\frac{1}{2} h g_{\mu \nu}^{(0)} \\
& \hat{h}_{\mu \nu} \equiv h_{\mu}{ }^{\alpha} h_{\alpha \nu}-\frac{1}{2} h h_{\mu \nu}+\frac{1}{8} h^{2} g_{\mu \nu}^{(0)}-\frac{1}{4} h_{\alpha \beta} h^{\alpha \beta} g_{\mu \nu}^{(0)} .
\end{aligned}
$$

From quantum field theory in curved spacetime considerations discussed above, we take the following action for the gravitational field:

$$
\begin{aligned}
S_{\mathrm{g}}\left[g_{\mu \nu}\right]= & \frac{1}{(16 \pi G)^{\frac{n-2}{2}}} \int d^{n} x \sqrt{-g(x)} R(x)+\frac{\alpha \bar{\mu}^{n-4}}{4(n-4)} \int d^{n} x \sqrt{-g(x)} \\
& \times\left\{3 R_{\mu \nu \alpha \beta}(x) R^{\mu \nu \alpha \beta}(x)-\left[1-360\left(\xi(n)-\frac{1}{6}\right)^{2}\right] R^{2}(x)\right\} .
\end{aligned}
$$

The first term is the classical Einstein-Hilbert action; the second term is the counterterm in four dimensions used to renormalize the divergent effective action. In this action $\ell_{\mathrm{P}}^{2}=16 \pi G_{\mathrm{N}}$, $\alpha=\left(2880 \pi^{2}\right)^{-1}$ and $\bar{\mu}$ is an arbitrary mass scale.

We are interested in computing the CTP effective action (189) for the matter action and when the field $\phi$ is initially in the Hartle-Hawking vacuum. This is equivalent to saying that the initial state of the field is described by a thermal density matrix at a finite temperature $T=T_{\mathrm{H}}$. The CTP effective action at finite temperature $T \equiv 1 / \beta$ for this model is given by (for details see [69, 70])

$$
S_{\text {eff }}^{\beta}\left[h_{\mu \nu}^{ \pm}\right]=S_{\mathrm{g}}\left[h_{\mu \nu}^{+}\right]-S_{\mathrm{g}}\left[h_{\mu \nu}^{-}\right]-\frac{i}{2} \operatorname{tr}\left\{\ln \bar{G}_{a b}^{\beta}\left[h_{\mu \nu}^{ \pm}\right]\right\},
$$

where \pm denote the forward and backward time path of the CTP formalism, and $\bar{G}_{a b}^{\beta}\left[h_{\mu \nu}^{ \pm}\right]$is the complete $2 \times 2$ matrix propagator ( $a$ and $b$ take \pm values: $G_{++}, G_{+-}$, and $G_{--}$correspond to the Feynman, Wightman, and Schwinger Green's functions respectively) with thermal boundary conditions for the differential operator $\sqrt{-g^{(0)}}\left(\square+V^{(1)}+V^{(2)}+\ldots\right)$. The actual form of $\bar{G}_{a b}^{\beta}$ cannot be explicitly given. However, it is easy to obtain a perturbative expansion in terms of $V_{a b}^{(k)}$, the $k$-order matrix version of the complete differential operator defined by $V_{ \pm \pm}^{(k)} \equiv \pm V_{ \pm}^{(k)}$ and $V_{ \pm \mp}^{(k)} \equiv 0$, and $G_{a b}^{\beta}$, the thermal matrix propagator for a massless scalar field in Schwarzschild spacetime. To second order $\bar{G}_{a b}^{\beta}$ reads

$$
\bar{G}_{a b}^{\beta}=G_{a b}^{\beta}-G_{a c}^{\beta} V_{c d}^{(1)} G_{d b}^{\beta}-G_{a c}^{\beta} V_{c d}^{(2)} G_{d b}^{\beta}+G_{a c}^{\beta} V_{c d}^{(1)} G_{d e}^{\beta} V_{e f}^{(1)} G_{f b}^{\beta}+\ldots
$$

Expanding the logarithm and dropping one term independent of the perturbation $h_{\mu \nu}^{ \pm}(x)$, the CTP effective action may be perturbatively written as

$$
\begin{aligned}
S_{\mathrm{eff}}^{\beta}\left[h_{\mu \nu}^{ \pm}\right]= & S_{\mathrm{g}}\left[h_{\mu \nu}^{+}\right]-S_{\mathrm{g}}\left[h_{\mu \nu}^{-}\right] \\
& +\frac{i}{2} \operatorname{tr}\left[V_{+}^{(1)} G_{++}^{\beta}-V_{-}^{(1)} G_{--}^{\beta}+V_{+}^{(2)} G_{++}^{\beta}-V_{-}^{(2)} G_{--}^{\beta}\right] \\
& -\frac{i}{4} \operatorname{tr}\left[V_{+}^{(1)} G_{++}^{\beta} V_{+}^{(1)} G_{++}^{\beta}+V_{-}^{(1)} G_{--}^{\beta} V_{-}^{(1)} G_{--}^{\beta}-2 V_{+}^{(1)} G_{+-}^{\beta} V_{-}^{(1)} G_{-+}^{\beta}\right]
\end{aligned}
$$


In computing the traces, some terms containing divergences are canceled using counterterms introduced in the classical gravitational action after dimensional regularization.

\subsubsection{Near flat case}

At this point we divide our considerations into two cases. In the far field limit $h_{\mu \nu}$ represent perturbations about flat space, i.e., $g_{\mu \nu}^{(0)}=\eta_{\mu \nu}$. The exact "unperturbed" thermal propagators for scalar fields are known, i.e., the Euclidean propagator with periodicity $\beta$. Using the Fouriertransformed form (those quantities are denoted with a tilde) of the thermal propagators $\tilde{G}_{a b}^{\beta}(k)$, the trace terms of the form $\operatorname{tr}\left[V_{a}^{(1)} G_{m n}^{\beta} V_{b}^{(1)} G_{r s}^{\beta}\right]$ can be written as [69, 70]

$$
\begin{aligned}
& \operatorname{tr}\left[V_{a}^{(1)} G_{m n}^{\beta} V_{b}^{(1)} G_{r s}^{\beta}\right]= \\
& \quad \int d^{n} x d^{n} x^{\prime} h_{\mu \nu}^{a}(x) h_{\alpha \beta}^{b}\left(x^{\prime}\right) \int \frac{d^{n} k}{(2 \pi)^{n}} \frac{d^{n} q}{(2 \pi)^{n}} e^{i k\left(x-x^{\prime}\right)} \tilde{G}_{m n}^{\beta}(k+q) \tilde{G}_{r s}^{\beta}(q) \mathrm{T}^{\mu \nu, \alpha \beta}(q, k),
\end{aligned}
$$

where the tensor $\mathrm{T}^{\mu \nu, \alpha \beta}(q, k)$ is defined in [69, 70] after an expansion in terms of a basis of 14 tensors [311, 312]. In particular, the last trace of Equation (195) may be split into two different kernels $\mathrm{N}^{\mu \nu, \alpha \beta}\left(x-x^{\prime}\right)$ and $\mathrm{D}^{\mu \nu, \alpha \beta}\left(x-x^{\prime}\right)$,

$$
\frac{i}{2} \operatorname{tr}\left[V_{+}^{(1)} G_{+-}^{\beta} V_{-}^{(1)} G_{-+}^{\beta}\right]=-\int d^{4} x d^{4} x^{\prime} h_{\mu \nu}^{+}(x) h_{\alpha \beta}^{-}\left(x^{\prime}\right)\left[\mathrm{D}^{\mu \nu, \alpha \beta}\left(x-x^{\prime}\right)+i \mathrm{~N}^{\mu \nu, \alpha \beta}\left(x-x^{\prime}\right)\right] .
$$

One can express the Fourier transforms of these kernels as

$$
\begin{gathered}
\tilde{\mathrm{N}}^{\mu \nu, \alpha \beta}(k)=\pi^{2} \int \frac{d^{4} q}{(2 \pi)^{4}}\left\{\theta\left(k^{o}+q^{o}\right) \theta\left(-q^{o}\right)+\theta\left(-k^{o}-q^{o}\right) \theta\left(q^{o}\right)+n_{\beta}\left(\left|q^{o}\right|\right)+n_{\beta}\left(\left|k^{o}+q^{o}\right|\right)\right. \\
\left.+2 n_{\beta}\left(\left|q^{o}\right|\right) n_{\beta}\left(\left|k^{o}+q^{o}\right|\right)\right\} \delta\left(q^{2}\right) \delta\left[(k+q)^{2}\right] \mathrm{T}^{\mu \nu, \alpha \beta}(q, k), \\
\begin{aligned}
\tilde{\mathrm{D}}^{\mu \nu, \alpha \beta}(k)=-i \pi^{2} \int \frac{d^{4} q}{(2 \pi)^{4}}\left\{\theta\left(k^{o}+q^{o}\right) \theta\left(-q^{o}\right)-\theta\left(-k^{o}-q^{o}\right) \theta\left(q^{o}\right)+\operatorname{sig}\left(k^{o}+q^{o}\right) n_{\beta}\left(\left|q^{o}\right|\right)\right. \\
\left.-\operatorname{sig}\left(q^{o}\right) n_{\beta}\left(\left|k^{o}+q^{o}\right|\right)\right\} \delta\left(q^{2}\right) \delta\left[(k+q)^{2}\right] \mathrm{T}^{\mu \nu, \alpha \beta}(q, k),
\end{aligned}
\end{gathered}
$$

respectively.

Using the property $\mathrm{T}^{\mu \nu, \alpha \beta}(q, k)=\mathrm{T}^{\mu \nu, \alpha \beta}(-q,-k)$, it is easy to see that the kernel $\mathrm{N}^{\mu \nu, \alpha \beta}\left(x-x^{\prime}\right)$ is symmetric and $\mathrm{D}^{\mu \nu, \alpha \beta}\left(x-x^{\prime}\right)$ is antisymmetric in its arguments; that is, $\mathrm{N}^{\mu \nu, \alpha \beta}(x)=\mathrm{N}^{\mu \nu, \alpha \beta}(-x)$ and $\mathrm{D}^{\mu \nu, \alpha \beta}(x)=-\mathrm{D}^{\mu \nu, \alpha \beta}(-x)$.

The physical meanings of these kernels can be extracted if we write the renormalized CTP effective action at finite temperature (195) in an influence-functional form [45, 133, 196, 197]. N, the imaginary part of the CTP effective action, can be identified with the noise kernel and D, the antisymmetric piece of the real part, with the dissipation kernel. Campos and $\mathrm{Hu}[69,70]$ have shown that these kernels identified as such indeed satisfy a thermal fluctuation-dissipation relation.

If we denote the difference and the sum of the perturbations $h_{\mu \nu}^{ \pm}$, defined along each branch $C_{ \pm}$of the complex time path of integration $C$, by $\left[h_{\mu \nu}\right] \equiv h_{\mu \nu}^{+}-h_{\mu \nu}^{-}$and $\left\{h_{\mu \nu}\right\} \equiv h_{\mu \nu}^{+}+h_{\mu \nu}^{-}$, respectively, the influence-functional form of the thermal CTP effective action may be written to 
second order in $h_{\mu \nu}$ as

$$
\begin{aligned}
S_{\text {eff }}^{\beta}\left[h_{\mu \nu}^{ \pm}\right] \simeq & \frac{1}{2\left(16 \pi G_{\mathrm{N}}\right)} \int d^{4} x d^{4} x^{\prime}\left[h_{\mu \nu}\right](x) \mathrm{L}_{(o)}^{\mu \nu, \alpha \beta}\left(x-x^{\prime}\right)\left\{h_{\alpha \beta}\right\}\left(x^{\prime}\right) \\
& +\frac{1}{2} \int d^{4} x\left[h_{\mu \nu}\right](x) T_{(\beta)}^{\mu \nu} \\
& +\frac{1}{2} \int d^{4} x d^{4} x^{\prime}\left[h_{\mu \nu}\right](x) \mathrm{H}^{\mu \nu, \alpha \beta}\left(x-x^{\prime}\right)\left\{h_{\alpha \beta}\right\}\left(x^{\prime}\right) \\
& -\frac{1}{2} \int d^{4} x d^{4} x^{\prime}\left[h_{\mu \nu}\right](x) \mathrm{D}^{\mu \nu, \alpha \beta}\left(x-x^{\prime}\right)\left\{h_{\alpha \beta}\right\}\left(x^{\prime}\right) \\
& +\frac{i}{2} \int d^{4} x d^{4} x^{\prime}\left[h_{\mu \nu}\right](x) \mathrm{N}^{\mu \nu, \alpha \beta}\left(x-x^{\prime}\right)\left[h_{\alpha \beta}\right]\left(x^{\prime}\right) .
\end{aligned}
$$

The first line is the Einstein-Hilbert action to second order in the perturbation $h_{\mu \nu}^{ \pm}(x) . \mathrm{L}_{(o)}^{\mu \nu, \alpha \beta}(x)$ is a symmetric kernel, i.e., $\mathrm{L}_{(o)}^{\mu \nu, \alpha \beta}(x)=\mathrm{L}_{(o)}^{\mu \nu, \alpha \beta}(-x)$. In the near flat case, its Fourier transform is given by

$$
\tilde{\mathrm{L}}_{(o)}^{\mu \nu, \alpha \beta}(k)=\frac{1}{4}\left[-k^{2} \mathrm{~T}_{1}^{\mu \nu, \alpha \beta}(q, k)+2 k^{2} \mathrm{~T}_{4}^{\mu \nu, \alpha \beta}(q, k)+\mathrm{T}_{8}^{\mu \nu, \alpha \beta}(q, k)-2 \mathrm{~T}_{13}^{\mu \nu, \alpha \beta}(q, k)\right] .
$$

The 14 elements of the tensor basis $\mathrm{T}_{i}^{\mu \nu, \alpha \beta}(q, k), i=1, \ldots, 14$, are defined in [311, 312]. The second is a local term, linear in $h_{\mu \nu}^{ \pm}(x)$. Only far away from the black hole it takes the form of the stress tensor of massless scalar particles at temperature $\beta^{-1}$, which has the form of a perfect fluid stress-energy tensor,

$$
T_{(\beta)}^{\mu \nu}=\frac{\pi^{2}}{30 \beta^{4}}\left[u^{\mu} u^{\nu}+\frac{1}{3}\left(\eta^{\mu \nu}+u^{\mu} u^{\nu}\right)\right],
$$

where $u^{\mu}$ is the four-velocity of the plasma and the factor $\frac{\pi^{2}}{30 \beta^{4}}$ is the familiar thermal energy density for massless scalar particles at temperature $\beta^{-1}$. In the far field limit, taking into account the four-velocity $u^{\mu}$ of the fluid, a manifestly Lorentz-covariant approach to thermal field theory may be used [371]. However, in order to simplify the involved tensorial structure, we work in the co-moving coordinate system of the fluid, where $u^{\mu}=(1,0,0,0)$. In the third line, the Fourier transform of the symmetric kernel $\mathrm{H}^{\mu \nu, \alpha \beta}(x)$ can be expressed as

$$
\begin{aligned}
\tilde{\mathrm{H}}^{\mu \nu, \alpha \beta}(k)= & -\frac{\alpha k^{4}}{4}\left\{\frac{1}{r^{2}} \ln \frac{\left|k^{2}\right|}{\mu^{2}} \mathrm{Q}^{\mu \nu, \alpha \beta}(k)+\frac{1}{3} \overline{\mathrm{Q}}^{\mu \nu, \alpha \beta}(k)\right\} \\
& +\frac{\pi^{2}}{180 \beta^{4}}\left\{-\mathrm{T}_{1}^{\mu \nu, \alpha \beta}(u, k)-2 \mathrm{~T}_{2}^{\mu \nu, \alpha \beta}(u, k)+\mathrm{T}_{4}^{\mu \nu, \alpha \beta}(u, k)+2 \mathrm{~T}_{5}^{\mu \nu, \alpha \beta}(u, k)\right\} \\
& +\frac{\xi}{96 \beta^{2}}\left\{k^{2} \mathrm{~T}_{1}^{\mu \nu, \alpha \beta}(u, k)-2 k^{2} \mathrm{~T}_{4}^{\mu \nu, \alpha \beta}(u, k)-\mathrm{T}_{8}^{\mu \nu, \alpha \beta}(u, k)+2 \mathrm{~T}_{13}^{\mu \nu, \alpha \beta}(u, k)\right\} \\
& +\pi \int \frac{d^{4} q}{(2 \pi)^{4}}\left\{\delta\left(q^{2}\right) n_{\beta}\left(\left|q^{o}\right|\right) \mathcal{P}\left[\frac{1}{(k+q)^{2}}\right]+\delta\left[(k+q)^{2}\right] n_{\beta}\left(\left|k^{o}+q^{o}\right|\right) \mathcal{P}\left[\frac{1}{q^{2}}\right]\right\} \mathrm{T}^{\mu \nu, \alpha \beta}(q, k),
\end{aligned}
$$

where $\mu$ is a simple redefinition of the renormalization parameter $\bar{\mu}$ given by

$$
\mu \equiv \bar{\mu} \exp \left(\frac{23}{15}+\frac{1}{2} \ln 4 \pi-\frac{1}{2} \gamma\right)
$$


and the tensors $\mathrm{Q}^{\mu \nu, \alpha \beta}(k)$ and $\overline{\mathrm{Q}}^{\mu \nu, \alpha \beta}(k)$ are defined by

$$
\begin{aligned}
\mathrm{Q}^{\mu \nu, \alpha \beta}(k)= & \frac{3}{2}\left\{\mathrm{~T}_{1}^{\mu \nu, \alpha \beta}(q, k)-\frac{1}{k^{2}} \mathrm{~T}_{8}^{\mu \nu, \alpha \beta}(q, k)+\frac{2}{k^{4}} \mathrm{~T}_{12}^{\mu \nu, \alpha \beta}(q, k)\right\} \\
& -\left[1-360\left(\xi-\frac{1}{6}\right)^{2}\right]\left\{\mathrm{T}_{4}^{\mu \nu, \alpha \beta}(q, k)+\frac{1}{k^{4}} \mathrm{~T}_{12}^{\mu \nu, \alpha \beta}(q, k)-\frac{1}{k^{2}} \mathrm{~T}_{13}^{\mu \nu, \alpha \beta}(q, k)\right\}, \\
\overline{\mathrm{Q}}^{\mu \nu, \alpha \beta}(k)= & {\left[1+576\left(\xi-\frac{1}{6}\right)^{2}-60\left(\xi-\frac{1}{6}\right)\left(1-36 \xi^{\prime}\right)\right] } \\
& \times\left\{\mathrm{T}_{4}^{\mu \nu, \alpha \beta}(q, k)+\frac{1}{k^{4}} \mathrm{~T}_{12}^{\mu \nu, \alpha \beta}(q, k)-\frac{1}{k^{2}} \mathrm{~T}_{13}^{\mu \nu, \alpha \beta}(q, k)\right\},
\end{aligned}
$$

respectively.

In the above and subsequent equations, we denote the coupling parameter in four dimensions $\xi(4)$ by $\xi$, and consequently $\xi^{\prime}$ means $d \xi(n) / d n$ evaluated at $n=4$. $\tilde{\mathrm{H}}^{\mu \nu, \alpha \beta}(k)$ is the complete contribution of a free massless quantum scalar field to the thermal graviton polarization tensor $[39,89,311,312]$, and it is responsible for the instabilities found in flat spacetime at finite temperature $[39,89,138,311,312]$. Note that the addition of the contribution of other kinds of matter fields to the effective action, even graviton contributions, does not change the tensor structure of these kernels, and only the overall factors are different to leading order [311, 312]. Equation (203) reflects the fact that the kernel $\tilde{\mathrm{H}}^{\mu \nu, \alpha \beta}(k)$ has thermal, as well as non-thermal, contributions. Note that it reduces to the first term in the zero temperature limit $(\beta \rightarrow \infty)$,

$$
\tilde{\mathrm{H}}^{\mu \nu, \alpha \beta}(k) \simeq-\frac{\alpha k^{4}}{4}\left\{\frac{1}{2} \ln \frac{\left|k^{2}\right|}{\mu^{2}} \mathrm{Q}^{\mu \nu, \alpha \beta}(k)+\frac{1}{3} \overline{\mathrm{Q}}^{\mu \nu, \alpha \beta}(k)\right\},
$$

and at high temperatures the leading term $\left(\beta^{-4}\right)$ may be written as

$$
\tilde{\mathrm{H}}^{\mu \nu, \alpha \beta}(k) \simeq \frac{\pi^{2}}{30 \beta^{4}} \sum_{i=1}^{14} \mathrm{H}_{i}(r) \mathrm{T}_{i}^{\mu \nu, \alpha \beta}(u, K),
$$

where we have introduced the dimensionless external momentum $K^{\mu} \equiv k^{\mu} /|\vec{k}| \equiv(r, \hat{k})$. The $\mathrm{H}_{i}(r)$ coefficients were first given in [311, 312] and generalized to the next-to-leading order $\beta^{-2}$ in [39, 89]. (They are given with the MTW sign convention in [69, 70].)

Finally, as defined above, $\mathrm{N}^{\mu \nu, \alpha \beta}(x)$ is the noise kernel representing random fluctuations of thermal radiance and $\mathrm{D}^{\mu \nu, \alpha \beta}(x)$ is the dissipation kernel, describing the dissipation of energy of the gravitational field.

\subsubsection{Near-horizon case}

In this case, since the perturbation is taken around the Schwarzschild spacetime, exact expressions for the corresponding unperturbed propagators $G_{a b}^{\beta}\left[h_{\mu \nu}^{ \pm}\right]$are not known. Therefore, apart from the approximation of computing the CTP effective action to certain order in perturbation theory, an appropriate approximation scheme for the unperturbed Green's functions is also required. This feature manifested itself in York's calculation of backreaction as well, where, in writing $\left\langle T_{\mu \nu}\right\rangle$ on the right-hand side of the semiclassical Einstein equation in the unperturbed Schwarzschild metric, he had to use an approximate expression for $\left\langle T_{\mu \nu}\right\rangle$ in the Schwarzschild metric, given by Page [287]. The additional complication here is that, while to obtain $\left\langle T_{\mu \nu}\right\rangle$ as in York's calculation, the knowledge of only the thermal Feynman Green's function is required, to calculate the CTP effective action one needs the knowledge of the full matrix propagator, which involves the Feynman, Schwinger and Wightman functions. 
It is indeed possible to construct the full thermal matrix propagator $G_{a b}^{\beta}\left[h_{\mu \nu}^{ \pm}\right]$based on Page's approximate Feynman Green's function by using identities relating the Feynman Green's function with the other Green's functions with different boundary conditions. One can then proceed to explicitly compute a CTP effective action and hence the influence functional based on this approximation. However, we desist from delving into such a calculation for the following reason. Our main interest in performing such a calculation is to identify and analyze the noise term, which is the new ingredient in the backreaction. We have mentioned that the noise term gives a stochastic contribution $\xi^{\mu \nu}$ to the Einstein-Langevin equation (15). We had also stated that this term is related to the variance of fluctuations in $T_{\mu \nu}$, i.e, schematically, to $\left\langle T_{\mu \nu}^{2}\right\rangle$. However, a calculation of $\left\langle T_{\mu \nu}^{2}\right\rangle$ in the Hartle-Hawking state in a Schwarzschild background using the Page approximation was performed by Phillips and $\mathrm{Hu}[304,305]$ and it was shown that, though the approximation is excellent as far as $\left\langle T_{\mu \nu}\right\rangle$ is concerned, it gives unacceptably large errors for $\left\langle T_{\mu \nu}^{2}\right\rangle$ at the horizon. In fact, similar errors will be propagated in the non-local dissipation term as well, because both terms originate from the same source, that is, they come from the last trace term in (195), which contains terms quadratic in the Green's function. However, the influence functional or CTP formalism itself does not depend on the nature of the approximation, so we will attempt to exhibit the general structure of the calculation without resorting to a specific form for the Green's function and conjecture on what is to be expected. A more accurate computation can be performed using this formal structure once a better approximation becomes available.

The general structure of the CTP effective action arising from the calculation of the traces in Equation (195) remains the same. But to write down explicit expressions for the non-local kernels, one requires the input of the explicit form of $G_{a b}^{\beta}\left[h_{\mu \nu}^{ \pm}\right]$in the Schwarzschild metric, which is not available in closed form. We can make some general observations about the terms in there. The first line containing $\mathrm{L}$ does not have an explicit Fourier representation as given in the far-field case; neither will $T_{(\beta)}^{\mu \nu}$ in the second line representing the zeroth-order contribution to $\left\langle T_{\mu \nu}\right\rangle$ have a perfect fluid form. The third and fourth terms containing the remaining quadratic component of the real part of the effective action will not have any simple or even complicated analytic form. The symmetry properties of the kernels $H^{\mu \nu, \alpha \beta}\left(x, x^{\prime}\right)$ and $D^{\mu \nu, \alpha \beta}\left(x, x^{\prime}\right)$ remain intact, i.e., they are respectively even and odd in $x, x^{\prime}$. The last term in the CTP effective action gives the imaginary part of the effective action and the kernel $N\left(x, x^{\prime}\right)$ is symmetric.

Continuing our general observations from this CTP effective action, using the connection between this thermal CTP effective action to the influence functional $[58,343]$ via an equation in the schematic form (24), we see that the nonlocal imaginary term containing the kernel $N^{\mu \nu, \alpha \beta}\left(x, x^{\prime}\right)$ is responsible for the generation of the stochastic noise term in the Einstein-Langevin equation and the real non-local term containing kernel $D^{\mu \nu, \alpha \beta}\left(x, x^{\prime}\right)$ is responsible for the non-local dissipation term. To derive the Einstein-Langevin equation we first construct the stochastic effective action (34). We then derive the equation of motion, as shown earlier in (36), by taking its functional derivative with respect to $\left[h_{\mu \nu}\right]$ and equating it to zero. With the identification of noise and dissipation kernels, one can write down a linear, non-local relation of the form

$$
N\left(t-t^{\prime}\right)=\int d\left(s-s^{\prime}\right) K\left(t-t^{\prime}, s-s^{\prime}\right) \gamma\left(s-s^{\prime}\right)
$$

where $D\left(t, t^{\prime}\right)=-\partial_{t^{\prime}} \gamma\left(t, t^{\prime}\right)$. This is the general functional form of a fluctuation-dissipation relation and $K(t, s)$ is called the fluctuation-dissipation kernel [45, 133, 196, 197]. In the present context this relation depicts the backreaction of thermal Hawking radiance for a black hole in quasi-equilibrium.

\subsubsection{Einstein-Langevin equation}

In this section we show how a semiclassical Einstein-Langevin equation can be derived from the previous thermal CTP effective action. This equation depicts the stochastic evolution of the 
perturbations of the black hole under the influence of the fluctuations of the thermal scalar field.

The influence functional $\mathcal{F}_{\mathrm{IF}} \equiv \exp \left(i S_{\mathrm{IF}}\right)$ previously introduced in Equation (23) can be written in terms of the the CTP effective action $S_{\text {eff }}^{\beta}\left[h_{\mu \nu}^{ \pm}\right]$derived in Equation (200) using Equation (24). The Einstein-Langevin equation follows from taking the functional derivative of the stochastic effective action (34) with respect to $\left[h_{\mu \nu}\right](x)$ and imposing $\left[h_{\mu \nu}\right](x)=0$. This leads to

$$
\begin{aligned}
& \frac{1}{16 \pi G_{\mathrm{N}}} \int d^{4} x^{\prime} \mathrm{L}_{(o)}^{\mu \nu, \alpha \beta}\left(x-x^{\prime}\right) h_{\alpha \beta}\left(x^{\prime}\right)+\frac{1}{2} T_{(\beta)}^{\mu \nu} \\
& +\int d^{4} x^{\prime}\left(\mathrm{H}^{\mu \nu, \alpha \beta}\left(x-x^{\prime}\right)-\mathrm{D}^{\mu \nu, \alpha \beta}\left(x-x^{\prime}\right)\right) h_{\alpha \beta}\left(x^{\prime}\right)+\xi^{\mu \nu}(x)=0,
\end{aligned}
$$

where

$$
\left\langle\xi^{\mu \nu}(x) \xi^{\alpha \beta}\left(x^{\prime}\right)\right\rangle_{j}=\mathrm{N}^{\mu \nu, \alpha \beta}\left(x-x^{\prime}\right) .
$$

In the far-field limit this equation should reduce to that obtained by Campos and $\mathrm{Hu}[69,70]$; for gravitational perturbations $h^{\mu \nu}$ defined in Equation (191) under the harmonic gauge $\bar{h}^{\mu \nu}{ }_{, \nu}=0$, their Einstein-Langevin equation is given by

$\square \bar{h}^{\mu \nu}(x)+\frac{1}{16 \pi G_{\mathrm{N}}^{2}}\left\{T_{(\beta)}^{\mu \nu}+2 P_{\rho \sigma, \alpha \beta} \int d^{4} x^{\prime}\left(\mathrm{H}^{\mu \nu, \alpha \beta}\left(x-x^{\prime}\right)-\mathrm{D}^{\mu \nu, \alpha \beta}\left(x-x^{\prime}\right)\right) \bar{h}^{\rho \sigma}\left(x^{\prime}\right)+2 \xi^{\mu \nu}(x)\right\}=0$,

where the tensor $P_{\rho \sigma, \alpha \beta}$ is given by

$$
P_{\rho \sigma, \alpha \beta}=\frac{1}{2}\left(\eta_{\rho \alpha} \eta_{\sigma \beta}+\eta_{\rho \beta} \eta_{\sigma \alpha}-\eta_{\rho \sigma} \eta_{\alpha \beta}\right) .
$$

The expression for $P_{\rho \sigma, \alpha \beta}$ in the near-horizon limit, of course, cannot be expressed in such a simple form. Note that this differential stochastic equation includes a non-local term responsible for the dissipation of the gravitational field and a noise-source term, which accounts for the fluctuations of the quantum field. Note also that this equation, in combination with the correlation for the stochastic variable (211) determines, the two-point correlation for the stochastic metric fluctuations $\left\langle\bar{h}_{\mu \nu}(x) \bar{h}_{\alpha \beta}\left(x^{\prime}\right)\right\rangle_{\xi}$ self-consistently.

As we have seen before and here, the Einstein-Langevin equation is a dynamical equation governing the dissipative evolution of the gravitational field under the influence of the fluctuations of the quantum field, which, in the case of black holes, takes the form of thermal radiance. From its form we can see that, even for the quasi-static case under study, the backreaction of Hawking radiation on the black-hole spacetime has an innate dynamical nature.

For the far-field case, making use of the explicit forms available for the noise and dissipation kernels, Campos and $\mathrm{Hu}[69,70]$ formally prove the existence of a fluctuation-dissipation relation at all temperatures between the quantum fluctuations of the thermal radiance and the dissipation of the gravitational field. They also show the formal equivalence of this method with linear-response theory for lowest order perturbations of a near-equilibrium system and how the response functions, such as the contribution of the quantum scalar field to the thermal graviton polarization tensor, can be derived. An important quantity not usually obtained in linear-response theory, but of equal importance, manifest in the CTP stochastic approach is the noise term arising from the quantum and statistical fluctuations in the thermal field. The example given in this section shows that the backreaction is intrinsically a dynamic process described (at this level of sophistication) by the Einstein-Langevin equation. By comparison, traditional linear response theory calculations cannot capture the dynamics as fully and thus cannot provide a complete description of the backreaction problem. 


\subsubsection{Comments}

As remarked earlier, except for the near-flat case, an analytic form of the Green's function is not available. Even the Page approximation [287], which gives unexpectedly good results for the stressenergy tensor, has been shown to fail in the fluctuations of the energy density [305]. Thus, using such an approximation for the noise kernel will give unreliable results for the Einstein-Langevin equation. If we confine ourselves to Page's approximation and derive the equation of motion without the stochastic term, we expect to recover York's semiclassical Einstein's equation, if one retains only the zeroth-order contribution, i.e, the first two terms in the expression for the CTP effective action in Equation (200). Thus, this offers a new route to arrive at York's semiclassical Einstein's equations. (Not only is it a derivation of York's result from a different point of view, but it also shows how his result arises as an appropriate limit of a more complete framework, i.e, it arises when one averages over the noise.) Another point worth noting is that a non-local dissipation term arises from the fourth term in Equation (200) in the CTP effective action, which is absent in York's treatment. This difference exists primarily due to the difference in the way backreaction is treated, at the level of iterative approximations on the equation of motion as in York, versus the treatment at the effective-action level, as in the influence-functional approach. In York's treatment, the Einstein tensor is computed to first order in perturbation theory, while $\left\langle T_{\mu \nu}\right\rangle$ on the right-hand side of the semiclassical Einstein equation is replaced by the zeroth-order term. In the influence-functional treatment the full effective action is computed to second order in perturbation and hence includes the higher-order non-local terms.

The other important conceptual point that comes to light from this new approach is that related to the fluctuation-dissipation relation. In the quantum Brownian motion analog (e.g., [45, 133, 196, 197] and references therein), the dissipation of the energy of the Brownian particle as it approaches equilibrium and the fluctuations at equilibrium are connected by the Fluctuation-Dissipation relation. Here the backreaction of quantum fields on black holes also consists of two forms - dissipation and fluctuation or noise - corresponding to the real and imaginary parts of the influence functional as embodied in the dissipation and noise kernels. A fluctuation-dissipation relation has been shown to exist for the near-flat case by Campos and $\mathrm{Hu}[69,70]$ and is expected to exist between the noise and dissipation kernels for the general case, as it is a categorical relation [45, 133, 183, 196, 197]. Martin and Verdaguer have also proved the existence of a fluctuation-dissipation relation when the semiclassical background is a stationary spacetime and the quantum field is in thermal equilibrium. Their result was then extended to a conformal field in a conformally-stationary background [257]. As discussed earlier, the existence of a fluctuation-dissipation relation for the black-hole case has previously been suggested by some authors previously [76, 268, 324, 325]. This relation and the relevant physical quantities contained therein, such as the black-hole susceptibility function, which characterizes the statistical mechanical and dynamical responses of a black hole interacting with its quantum field environment, will allow us to study the nonequilibrium thermodynamic properties of the black hole and through it, perhaps, the microscopic structure of spacetime.

There are limitations of a technical nature in the quasi-static case studied, as mentioned above, i.e., there is no reliable approximation to the Schwarzschild thermal Green's function to explicitly compute the noise and dissipation kernels. Another technical limitation of this example is the following: although we have allowed for backreaction effects to modify the initial state in the sense that the temperature of the Hartle-Hawking state gets affected by the backreaction, our analysis is essentially confined to a Hartle-Hawking thermal state of the field. It does not directly extend to a more general class of states, for example to the case in which the initial state of the field is in the Unruh vacuum. To study the dynamics of a radiating black hole under the influence of a quantum field and its fluctuations a different model and approach are needed, which we now discuss. 


\subsection{Metric fluctuations of an evaporating black hole}

At the semiclassical gravity level of description, black-hole evaporation results from the backreaction of particle production in Hawking effect. This is believed to be valid at least before the Planckian scale is reached $[18,260]$. However, as is explained above, semiclassical gravity [34, 110, 362] is a mean-field description that neglects the fluctuations of spacetime geometry. A number of studies have suggested the existence of large fluctuations near black-hole horizons [80, 255, 335, 336] (and even instabilities [264]) with characteristic time scales much shorter than the black hole evaporation time. For example, Casher et al. [80] and Sorkin [336, 338] have concentrated on the issue of fluctuations of the horizon induced by a fluctuating metric. Casher et al. [80] consider the fluctuations of the horizon induced by the "atmosphere" of high angular-momentum particles near the horizon, while Sorkin [336, 338] calculates fluctuations of the shape of the horizon induced by the quantum-field fluctuations under a Newtonian approximation. A relativistic generalization of this vein is given by Marolf [255]. Both groups of authors came to the conclusion that horizon fluctuations become large at scales much larger than the Planck scale (note Ford and Svaiter [114] later presented results contrary to this claim). Though these works do deal with backreaction, the fluctuations considered do not arise as an explicit stochastic noise term as in stochastic gravity. Either states, which are singular on the horizon (such as the Boulware vacuum for Schwarzschild spacetime), were explicitly considered or fluctuations were computed with respect to those states and found to be large near the horizon. Whether these huge fluctuations are of a generic nature or an artifact from the consideration of states singular on the horizon is an issue that deserves further investigation. By contrast, the fluctuations for states regular on the horizon were estimated in [377] and found to be small even when integrated over a time on the order of the evaporation time. These apparently-contradictory claims and the fact that most claims on black-hole horizon fluctuations were based on qualitative arguments and/or semi-quantitative estimates indicate that a more quantitative and self-consistent description is needed. This is what stochastic-gravity theory can provide.

Such a program of research has been pursued rigorously by Hu and Roura (HR) [200, 202] In contrast to the claims made before, they find that even for states regular on the horizon the accumulated fluctuations become significant by the time the black-hole mass has changed substantially, but well before reaching the Planckian regime. This result is different from those obtained in prior studies, but in agreement with earlier work by Bekenstein [24]. The apparent difference from the conclusions drawn in the earlier work of $\mathrm{Hu}$, Raval and Sinha [199], which was also based on stochastic gravity, will be explained later. We begin with the evolution of the mean geometry.

\subsubsection{Evolution of the mean geometry of an evaporating black hole}

Backreaction of the Hawking radiation emitted by the black hole on the dynamics of spacetime geometry has been studied in some detail for spherically-symmetric black holes [18, 260]. For a general spherically-symmetric metric there always exists a system of coordinates in which it takes the form

$$
d s^{2}=-e^{2 \psi(v, r)}\left(1-\frac{2 m(v, r)}{r}\right) d v^{2}+2 e^{\psi(v, r)} d v d r+r^{2}\left(d \theta^{2}+\sin ^{2} \theta d \varphi^{2}\right) .
$$

In general this metric exhibits an apparent horizon, at which the expansion of the outgoing radial null geodesics vanishes and which separates regions with positive and negative expansion for those geodesics, at those radii that correspond to (odd degree) zeroes of the $v v$ metric component. We mark the location of the apparent horizon by $r_{A H}(v)=2 M(v)$, where $M(v)$ satisfies the equation $2 m(2 M(v), v)=2 M(v)$.

The non-zero components of the semiclassical Einstein equation associated with the metric in 
Equation (214) become

$$
\begin{aligned}
& \frac{\partial m}{\partial v}=4 \pi r^{2}\left\langle T_{v}^{r}\right\rangle, \\
& \frac{\partial m}{\partial r}=-4 \pi r^{2}\left\langle T_{v}^{v}\right\rangle, \\
& \frac{\partial \psi}{\partial r}=4 \pi r\left\langle T_{r r}\right\rangle,
\end{aligned}
$$

where in the above and henceforth we use $\left\langle T_{\mu \nu}\right\rangle$ to denote the renormalized or regularized vacuum expectation value of the stress energy tensor $\left\langle\hat{T}_{\mu \nu}[g]\right\rangle_{\text {ren }}$ and employ Planckian units (with $m_{p}^{2}=1$ ).

Solving Equations (215)-(217) is not easy. However, one can introduce a useful adiabatic approximation in the regime, in which the mass of the black hole is much larger than the Planck mass, which is, in any case, a necessary condition for the semiclassical treatment to be valid. What this entails is that when $M \gg 1$ (remember that we are using Planckian units) for each value of $v$ one can simply substitute $\left\langle T_{\mu \nu}\right\rangle$ by its "parametric value" - by this we mean the expectation value of the stress-energy tensor of the quantum field in a Schwarzschild black hole with a mass corresponding to $M(v)$ evaluated at that value of $v$. This is in contrast to its dynamical value, which should be determined by solving self-consistently the semiclassical Einstein equation for the spacetime metric and the equations of motion for the quantum matter fields. This kind of approximation introduces errors of higher order in $L_{H} \equiv B / M^{2}$ ( $B$ is a dimensionless parameter that depends on the number of massless fields and their spins and accounts for their corresponding grey-body factors; it has been estimated to be of order $10^{-4}$ [285]), which are very small for black holes well above Planckian scales. These errors are due to the fact that $M(v)$ is not constant and that, even for a constant $M(v)$, the resulting static geometry is not exactly Schwarzschild because the vacuum polarization of the quantum fields gives rise to a non-vanishing $\left\langle\hat{T}_{a b}[g]\right\rangle_{r e n}[381]$.

The expectation value of the stress tensor for a Schwarzschild spacetime has been found to correspond to a thermal flux of radiation (with $\left\langle T_{v}^{r}\right\rangle=L_{H} /\left(4 \pi r^{2}\right)$ ) for large radii and of order $L_{H}$ near the horizon $[8,75,177,178,287]$. This shows the consistency of the adiabatic approximation for $L_{H} \ll 1$ : the right-hand side of Equations (215)-(217) contains terms of order $L_{H}$ and higher, so that the derivatives of $m(v, r)$ and $\psi(v, r)$ are indeed small. We note that the natural quantum state for a black hole formed by gravitational collapse is the Unruh vacuum, which corresponds to the absence of incoming radiation far from the horizon. The expectation value of the stress-tensor operator for that state is finite on the future horizon of Schwarzschild, which is the relevant one when identifying a region of the Schwarzschild geometry with the spacetime outside the collapsing matter for a black hole formed by gravitational collapse.

One can use the $v$ component of the stress-energy conservation equation

$$
\frac{\partial\left(r^{2}\left\langle T_{v}^{r}\right\rangle\right)}{\partial r}+r^{2} \frac{\partial\left\langle T_{v}^{v}\right\rangle}{\partial v}=0
$$

to relate the $\left\langle T_{v}^{r}\right\rangle$ components on the horizon and far from it. Integrating Equation (218) radially, one gets

$$
\left(r^{2}\left\langle T_{v}^{r}\right\rangle\right)(r=2 M(v), v)=\left(r^{2}\left\langle T_{v}^{r}\right\rangle\right)(r \approx 6 M(v), v)+O\left(L_{H}^{2}\right),
$$

where we considered a radius sufficiently far from the horizon, but not arbitrarily far, i.e., $2 M(v) \ll$ $r \ll M(v) / L_{H}$. Hence, Equation (219) relates the positive-energy flux radiated away far from the horizon and the negative-energy flux crossing the horizon. Taking into account this connection between energy fluxes and evaluating Equation (215) on the apparent horizon, we finally get the equation governing the evolution of its size:

$$
\frac{d M}{d v}=-\frac{B}{M^{2}}
$$


Unless $M(v)$ is constant, the event horizon and the apparent horizon do not coincide. However, in the adiabatic regime their radii are related, differing by a quantity of higher order in $L_{H}$ : $r_{E H}(v)=r_{A H}(v)\left(1+O\left(L_{H}\right)\right)$.

\subsubsection{Spherically-symmetric induced fluctuations}

We now consider metric fluctuations around a background metric $g_{a b}$ that corresponds to a given solution of semiclassical gravity. Their dynamics are governed by the Einstein-Langevin equation $[73,192,206,248]$

$$
G_{a b}^{(1)}[g+h]=\kappa\left\langle\hat{T}_{a b}^{(1)}[g+h]\right\rangle_{r e n}+\kappa \xi_{a b}[g] .
$$

(The superindex (1) indicates that only the terms linear in the metric perturbations should be considered.) Here $\xi_{a b}$ is a Gaussian stochastic source with vanishing expectation value and correlation function $\left\langle\xi_{a b}(x) \xi_{c d}\left(x^{\prime}\right)\right\rangle_{\xi}=(1 / 2)\left\langle\left\{\hat{t}_{a b}(x), \hat{t}_{c d}\left(x^{\prime}\right)\right\}\right\rangle$ (with $\left.\hat{t}_{a b} \equiv \hat{T}_{a b}-\left\langle\hat{T}_{a b}\right\rangle\right)$ known as the noise kernel and denoted by $N_{a b c d}\left(x, x^{\prime}\right)$.

As explained earlier, the symmetrized two-point function consists of two contributions: intrinsic and induced fluctuations. The intrinsic fluctuations are a consequence of the quantum width of the initial state of the metric perturbations; they are obtained in stochastic gravity by averaging over the initial conditions for the solutions of the homogeneous part of Equation (221), distributed according to the reduced Wigner function associated with the initial quantum state of the metric perturbations. On the other hand, the induced fluctuations are due to the quantum fluctuations of the matter fields interacting with the metric perturbations; they are obtained by solving the Einstein-Langevin equation using a retarded propagator with vanishing initial conditions.

In this section we study the spherically-symmetric sector, i.e., the monopole contribution, which corresponds to $l=0$, in a multipole expansion in terms of spherical harmonics $Y_{l m}(\theta, \phi)$, of metric fluctuations for an evaporating black hole. Restricting one's attention to the sphericallysymmetric sector of metric fluctuations necessarily implies a partial description of the fluctuations because, contrary to the case for semiclassical-gravity solutions, even if one starts with sphericallysymmetric initial conditions, the stress-tensor fluctuations will induce fluctuations involving higher multipoles. Thus, the multipole structure of the fluctuations is far richer than that of sphericallysymmetric semiclassical-gravity solutions, but this also means that obtaining a complete solution (including all multipoles) for fluctuations, rather than the mean value, is much more difficult. For spherically-symmetric fluctuations only induced fluctuations are possible. The fact that intrinsic fluctuations cannot exist can be clearly seen if one neglects vacuum-polarization effects, since Birkhoff's theorem forbids the existence of spherically-symmetric free metric perturbations in the exterior vacuum region of a spherically-symmetric black hole that keep the ADM mass constant. (This fact rings an alarm in the approach taken in [383] to the black-hole fluctuation problem. The degrees of freedom corresponding to spherically-symmetric perturbations are constrained by the Hamiltonian and momentum constraints both at the classical and quantum level. Therefore, they will not exhibit quantum fluctuations unless they are coupled to a quantum matter field.) Even when vacuum-polarization effects are included, spherically-symmetric perturbations, characterized by $m(v, r)$ and $\psi(v, r)$, are not independent degrees of freedom. This follows from Equations (215)(217), which can be regarded as constraint equations.

Considering only spherical-symmetry fluctuations is a simplification but it should be emphasized that it gives more accurate results than two-dimensional dilation-gravity models resulting from simple dimensional reduction $[249,341,349]$. This is because we project the solutions of the Einstein-Langevin equation just at the end, rather than considering only the contribution of the $s$-wave modes to the classical action for both the metric and the matter fields from the very beginning. Hence, an infinite number of modes for the matter fields with $l \neq 0$ contribute to the 
$l=0$ projection of the noise kernel, whereas only the $s$-wave modes for each matter field would contribute to the noise kernel if dimensional reduction had been imposed right from the start, as done in $[289,290,291]$ as well as in studies of two-dimensional dilation-gravity models.

The Einstein-Langevin equation for the spherically-symmetric sector of metric perturbations can be obtained by considering linear perturbations of $m(v, r)$ and $\psi(v, r)$, projecting the stochastic source that accounts for the stress-tensor fluctuations to the $l=0$ sector, and adding it to the right-hand side of Equations (215)-(217). We will focus our attention on the equation for the evolution of $\eta(v, r)$, the perturbation of $m(v, r)$ :

$$
\frac{\partial(m+\eta)}{\partial v}=-\frac{B}{(m+\eta)^{2}}+4 \pi r^{2} \xi_{v}^{r}+O\left(L_{H}^{2}\right),
$$

which reduces, after neglecting terms of order $L_{H}^{2}$ or higher, to the following equation to linear order in $\eta$ :

$$
\frac{\partial \eta}{\partial v}=\frac{2 B}{m^{3}} \eta+4 \pi r^{2} \xi_{v}^{r} .
$$

It is important to emphasize that in Equation (222) we assumed that the change in time of $\eta(v, r)$ is sufficiently slow for the adiabatic approximation, employed in the previous section to obtain the mean evolution of $m(v, r)$, to also be applied to the perturbed quantity $m(v, r)+\eta(v, r)$. This is guaranteed as long as the term corresponding to the stochastic source is of order $L_{H}$ or higher, a point that will be discussed below.

A more serious issue raised by HR is that in most previous investigations [24, 377] of the problem of metric fluctuations driven by quantum matter field fluctuations of states regular on the horizon (as far as the expectation value of the stress tensor is concerned) most authors assumed the existence of correlations between the outgoing energy flux far from the horizon and a negative energy flux crossing the horizon. (See, however, [290, 291], in which those correlators were shown to vanish in an effectively two-dimensional model.) In semiclassical gravity, using energy conservation arguments, such correlations have been confirmed for the expectation value of the energy fluxes, provided that the mass of the black hole is much larger than the Planck mass. However, a more careful analysis by HR shows that no such simple connection exists for energy flux fluctuations. It also reveals that the fluctuations on the horizon are in fact divergent. This requires that one modify the classical picture of the event horizon from a sharply defined three-dimensional hypersurface to that possessing a finite width, i.e., a fluctuating geometry. One needs to find an appropriate way of probing the metric fluctuations near the horizon and extracting physically meaningful information. It also testifies to the necessity of a complete reexamination of all cases afresh and that an evaluation of the noise kernel near the horizon seems unavoidable for the consideration of fluctuations and backreaction issues.

Having registered this cautionary note, $\mathrm{Hu}$ and Roura [202] first make the assumption that a relation between the fluctuations of the fluxes exists, so as to be able to compare with earlier work. They then show that this relation does not hold and discuss the essential elements required in understanding not only the mathematical theory but also the operational meaning of metric fluctuations.

\section{Case 1: Assuming that there is a relation between fluctuations}

Since the generation of Hawking radiation is especially sensitive to what happens near the horizon, from now on we will concentrate on the metric perturbations near the horizon and consider $\eta(v)=$ $\eta(v, 2 M(v))$. This means that possible effects on the Hawking radiation due to the fluctuations of the potential barrier for the radial mode functions will be missed by our analysis. Assuming that the fluctuations of the energy flux crossing the horizon and those far from it are exactly correlated, 
from Equation (223) we have

$$
\frac{d \eta(v)}{d v}=\frac{2 B}{M^{3}(v)} \eta(v)+\xi(v)
$$

where $\xi(v) \equiv 4 \pi\left(r^{2} \xi_{v}^{r}\right)(v, r \approx 6 M(v))$. The correlation function for the spherically-symmetric fluctuation $\xi(v)$ is determined by the integral over the whole solid angle of the $N_{v v}^{r r}$ component of the noise kernel, which is given by $(1 / 2)\left\langle\left\{\hat{t}_{v}^{r}(x), \hat{t}_{v}^{r}\left(x^{\prime}\right)\right\}\right\rangle$. The $l=0$ fluctuations of the energy flux of Hawking radiation far from a black hole formed by gravitational collapse, characterized also by $(1 / 2)\left\langle\left\{\hat{t}_{v}^{r}(x), \hat{t}_{v}^{r}\left(x^{\prime}\right)\right\}\right\rangle$ averaged over the whole solid angle, have been studied in [377]. Its main features are a correlation time of order $M$ and a characteristic fluctuation amplitude of order $\epsilon_{0} / M^{4}$ (this is the result of smearing the stress tensor two-point function, which diverges in the coincidence limit, over a period of time on the order of the correlation time). The order of magnitude of $\epsilon_{0}$ has been estimated to lie between $0.1 B$ and $B$ [24,377]. For simplicity, we will consider quantities smeared over a time of order $M$. We can then introduce the Markovian approximation $\left(\epsilon_{0} / M^{3}(v)\right) \delta\left(v-v^{\prime}\right)$, which coarse-grains the information on features corresponding to time scales shorter than the correlation time $M$. Under those conditions $r^{2} \xi_{v}^{r}$ is of order $1 / M^{2}$ and, thus, the adiabatic approximation made when deriving Equation (222) is justified.

The stochastic equation (224) for $\eta$ can be solved in the usual way and the correlation function for $\eta(v)$ can then be computed. Alternatively, one can obtain an equation for $\left\langle\eta^{2}(v)\right\rangle_{\xi}$ by first multiplying Equation (224) by $\eta(v)$ and then taking the expectation value. This brings out a term $\langle\eta(v) \xi(v)\rangle_{\xi}$ on the right-hand side. For delta-correlated noise (the Stratonovich prescription is the appropriate one here), it is equal to one half the time-dependent coefficient multiplying the delta function $\delta\left(v-v^{\prime}\right)$ in the correlator $\left\langle\xi(v) \xi\left(v^{\prime}\right)\right\rangle_{\xi}$, which is given by $\epsilon_{0} / M^{3}(v)$ in our case. Finally, changing from the $v$ coordinate to the mass function $M(v)$ for the background solution, we obtain

$$
\frac{d}{d M}\left\langle\eta^{2}(M)\right\rangle_{\xi}=-\frac{4}{M}\left\langle\eta^{2}(M)\right\rangle_{\xi}-\frac{\left(\epsilon_{0} / B\right)}{M} .
$$

The solutions of this equation are given by

$$
\left\langle\eta^{2}(M)\right\rangle_{\xi}=\left\langle\eta^{2}\left(M_{0}\right)\right\rangle_{\xi}\left(\frac{M_{0}}{M}\right)^{4}+\frac{\epsilon_{0}}{4 B}\left[\left(\frac{M_{0}}{M}\right)^{4}-1\right] .
$$

Provided that the fluctuations at the initial time corresponding to $M=M_{0}$ are negligible (much smaller than $\sqrt{\epsilon_{0} / 4 B} \sim 1$ ), the fluctuations become comparable to the background solution when $M \sim M_{0}^{2 / 3}$. Note that fluctuations of the horizon radius of order one in Planckian units do not correspond to Planck-scale physics because near the horizon $\Delta R=r-2 M$ corresponds to a physical distance $L \sim \sqrt{M \Delta R}$, as can be seen from the line element for Schwarzschild, $d s^{2}=-(1-2 M / r) d t^{2}+(1-2 M / r)^{-1} d r^{2}+r^{2}\left(d \theta^{2}+\sin ^{2} \theta d \varphi^{2}\right)$, by considering pairs of points at constant $t$. So $\Delta R \sim 1$ corresponds to $L \sim \sqrt{M}$, whereas a physical distance of order one is associated with $\Delta R \sim 1 / M$, which corresponds to an area change of order one for spheres with those radii. One can, therefore, have initial fluctuations of the horizon radius of order one for physical distances well above the Planck length for a black hole with a mass much larger than the Planck mass. One expects that the fluctuations for states that are regular on the horizon correspond to physical distances not much larger than the Planck length, so that the horizon-radius fluctuations would be much smaller than one for sufficiently large black hole masses. Nevertheless, that may not be the case when dealing with states, which are singular on the horizon, with estimated fluctuations of order $M^{1 / 3}$ or even $\sqrt{M}[80,255,264]$.

The result of HR for the growth of the fluctuations in size of the black-hole horizon agrees with the result obtained by Bekenstein in [24] and implies that, for a sufficiently massive black hole (a few solar masses or a supermassive black hole), the fluctuations become important before the Planckian regime is reached. 
This growth of the fluctuations, which was found by Bekenstein and confirmed here via the Einstein-Langevin equation, seems to be in conflict with the estimate given by $\mathrm{Wu}$ and Ford in [377]. According to their estimate, the accumulated mass fluctuations over a period on the order of the black hole evaporation time $\left(\Delta t \sim M_{0}^{3}\right)$ would be on the order of the Planck mass. The discrepancy is due to the fact that the first term on the right-hand side of Equation (224), which corresponds to the perturbed expectation value $\left\langle\hat{T}_{a b}^{(1)}[g+h]\right\rangle_{\text {ren }}$ in Equation (221), was not taken into account in [377]. The larger growth obtained here is a consequence of the secular effect of that term, which builds up in time (slowly at first, during most of the evaporation time, and becoming more significant at late times when the mass has changed substantially) and reflects the unstable nature of the background solution for an evaporating black hole.

As for the relation between HR's results reported here and earlier results of $\mathrm{Hu}$, Raval and Sinha in [199], there should not be any discrepancy, since both adopted the stochastic gravity framework and performed their analysis based on the Einstein-Langevin equation. The claim in [199] was based on a qualitative argument that focused on the dynamics of the stochastic source alone. If one adds in the consideration that the perturbations around the mean are unstable for an evaporating black hole, their results agree.

All this can be qualitatively understood as follows. Consider an evaporating black hole with initial mass $M_{0}$ and suppose that the initial mass is perturbed by an amount $\delta M_{0}=1$. The mean evolution for the perturbed black hole (without taking into account any fluctuations) leads to a mass perturbation that grows like $\delta M=\left(M_{0} / M\right)^{2} \delta M_{0}=\left(M_{0} / M\right)^{2}$, so that it becomes comparable to the unperturbed mass $M$ when $M \sim M_{0}^{2 / 3}$, which coincides with the result obtained above. Such a coincidence has a simple explanation: the fluctuations of the Hawking flux, which are on the order of the Planck mass, slowly accumulated during most of the evaporating time, as found by $\mathrm{Wu}$ and Ford, and gave a dispersion of that order for the mass distribution at the time when the instability of the small perturbations around the background solution start to become significant.

\section{Case 2: When no such relation exists and the consequences}

For conformal fields in two-dimensional spacetimes, HR shows that the correlations between the energy flux crossing the horizon and the flux far from it vanish. The correlation function for the outgoing and ingoing null-energy fluxes in an effectively two-dimensional model is explicitly computed in [290, 291] and is also found to vanish. On the other hand, in four dimensions the correlation function does not vanish in general and correlations between outgoing and ingoing fluxes do exist near the horizon (at least partially).

For black-hole masses much larger than the Planck mass one can use the adiabatic approximation for the background mean evolution. Therefore, to lowest order in $L_{H}$ one can compute the fluctuations of the stress tensor in Schwarzschild spacetime. In Schwarzschild, the amplitude of the fluctuations of $r^{2}\left\langle T_{v}^{r}\right\rangle$ far from the horizon is of order $1 / M^{2}\left(=M^{2} / M^{4}\right)$ when smearing over a correlation time of order $M$, which one can estimate for a hot thermal plasma in flat space [69, 70] (see also [377] for a computation of the fluctuations of $r^{2}\left\langle T_{v}^{r}\right\rangle$ far from the horizon). The amplitude of the fluctuations of $r^{2}\left\langle T_{v}^{r}\right\rangle$ is, thus, of the same order as its expectation value. However, their derivatives with respect to $v$ are rather different: since the characteristic variation times for the expectation value and the fluctuations are $M^{3}$ and $M$, respectively, $\partial\left(r^{2}\left\langle T_{v}^{r}\right\rangle\right) / \partial v$ is of order $1 / M^{5}$, whereas $\partial\left(r^{2} \xi_{v}^{r}\right) / \partial v$ is of order $1 / M^{3}$. This implies an additional contribution of order $L_{H}$ due to the second term in Equation (218) if one radially integrates the same equation applied to stress-tensor fluctuations (the stochastic source in the Einstein-Langevin equation). Hence, in contrast to the case of the mean value, the contribution from the second term in Equation (218) cannot be neglected when radially integrating, since it is of the same order as the contributions from the first term, and one can no longer obtain a simple relation between the outgoing energy flux far from the horizon and the energy flux crossing the horizon. 
What then? Without this convenience (which almost all earlier researchers have taken for granted), to get a more precise depiction we need to compute the noise kernel near the horizon. However, as shown by $\mathrm{Hu}$ and Phillips earlier [305] when they examine the coincidence limit of the noise kernel and confirmed by the careful analysis of HR using smearing functions [202], the noise kernel smeared over the horizon is divergent and so are the induced metric fluctuations. Hence, one cannot study the fluctuations of the horizon as a three-dimensional hypersurface for each realization of the stochastic source because the amplitude of the fluctuations is infinite, even when restricting one's attention to the $l=0$ sector. Instead, one should regard the horizon as possessing a finite effective width due to quantum fluctuations. In order to characterize its width one must find a sensible way of probing the metric fluctuations near the horizon and extracting physically-meaningful information, such as their effect on the Hawking radiation emitted by the black hole. How to probe metric fluctuations is an issue at the root base, which needs be dealt with in all discussions of metric fluctuations.

\subsubsection{Summary and prospects}

The work of HR [202], based on the stochastic-gravity program, found that the sphericallysymmetric fluctuations of the horizon size of an evaporating black hole become important at late times, and even comparable to its mean value when $M \sim M_{0}^{2 / 3}$, where $M_{0}$ is the mass of the black hole at some initial time when the fluctuations of the horizon radius are much smaller than the Planck length (remember that for large black-hole masses this can still correspond to physical distances much larger than the Planck length, as explained in Section 4). This is consistent with the result previously obtained by Bekenstein in [24].

It is important to realize that, for a sufficiently massive black hole, the fluctuations become significant well before the Planckian regime is reached. More specifically, for a solar-mass black hole, they become comparable to the mean value when the black-hole radius is on the order of $10 \mathrm{~nm}$, whereas for a supermassive black hole with $M \sim 10^{7} M_{\odot}$, that happens when the radius reaches a size on the order of $1 \mathrm{~mm}$. One expects that in those circumstances the low-energy effective-field theory approach of stochastic gravity should provide a reliable description.

Due to the nonlinear nature of the backreaction equations, such as Equation (222), the fact that the fluctuations of the horizon size can grow and become comparable to the mean value implies non-negligible corrections to the dynamics of the mean value itself. This can be seen by expanding Equation (222) (evaluated on the horizon) in powers of $\eta$ and taking the expectation value. Through order $\eta^{2}$ we get

$$
\begin{aligned}
\frac{d\left(M(v)+\langle\eta(v)\rangle_{\xi}\right)}{d v} & =-\left\langle\frac{B}{(M(v)+\eta(v))^{2}}\right\rangle_{\xi} \\
& =-\frac{B}{M^{2}(v)}\left[1-\frac{2}{M(v)}\langle\eta(v)\rangle_{\xi}+\frac{3}{M^{2}(v)}\left\langle\eta^{2}(v)\right\rangle_{\xi}+O\left(\frac{\eta^{3}}{M^{3}}\right)\right] .
\end{aligned}
$$

When the fluctuations become comparable to the mass itself, the third term (and higher-order terms) on the right-hand side is no longer negligible and we get non-trivial corrections to Equation (220) for the dynamics of the mean value. These corrections can be interpreted as higher-order radiative corrections to semiclassical gravity that include the effects of metric fluctuations on the evolution of the mean value.

Finally, we remark on the relation of this finding to earlier well-known results. Does the existence of significant deviations for the mean evolution mentioned above invalidate the earlier results by Bardeen and Massar based on semiclassical gravity in [18, 260]? First, those deviations start to become significant only after a period on the order of the evaporation time, when the mass of the black hole has decreased substantially. Second, since fluctuations were not considered 
in those references, a direct comparison cannot be established. Nevertheless, we can compare the average of the fluctuating ensemble. Doing so exhibits an evolution that deviates significantly when the fluctuations become important. However, if one considers a single member of the ensemble at that time, its evolution will be accurately described by the corresponding semiclassical gravity solution until the fluctuations around that particular solution become important again, after a period on the order of the evaporation time associated with the new initial value of the mass at that time.

\subsection{Other work on metric fluctuations but without backreaction}

In closing we mention some work on metric fluctuations where no backreaction is considered. Barrabes et al. [20, 21] have considered the propagation of null rays and massless fields in a black hole fluctuating geometry and have shown that the stochastic nature of the metric leads to a modified dispersion relation and helps to confront the trans-Planckian frequency problem. However, in this case the stochastic noise is put in by hand and does not naturally arise from coarse-graining as in a quantum open systems approach, in terms of which stochastic gravity can be interpreted. It also does not take backreaction into account. It will be interesting to explore how a stochastic black hole metric, arising as a solution to the Einstein-Langevin equation, hence fully incorporating backreaction, would affect the trans-Planckian problem.

As mentioned earlier, Ford and his collaborators [114, 115, 377] have also explored the issue of metric fluctuations in detail and in particular have studied the fluctuations of the black hole horizon induced by metric fluctuations. However, the fluctuations they considered are in the context of a fixed background and do not relate to the backreaction.

Another work on metric fluctuations with no backreaction is that of $\mathrm{Hu}$ and Shiokawa [205], who study effects associated with electromagnetic wave propagation in a Robertson-Walker universe and Schwarzschild spacetime with a small amount of given metric stochasticity. They find that time-independent randomness can decrease the total luminosity of Hawking radiation due to multiple scattering of waves outside the black hole and gives rise to event horizon fluctuations and fluctuations in the Hawking temperature. The stochasticity in the background metric in their work is assumed rather than derived (as induced by quantum-field fluctuations). But it is interesting to compare their results with those obtained in stochastic gravity with backreaction, as one can begin to get a sense of the different sources of stochasticity and their weights (see, e.g., [187] for a list of possible sources of stochasticity.)

In a subsequent paper, Shiokawa [328] shows that the scalar and spinor waves in a stochastic spacetime behave similarly to the electrons in a disordered system. Viewing this as a quantumtransport problem, he expresses the conductance and its fluctuations in terms of a nonlinear sigma model in the closed time-path formalism and shows that the conductance fluctuations are universal, independent of the volume of the stochastic region and the amount of stochasticity. This result has significant importance in characterizing the mesoscopic behavior of spacetimes resting between the semiclassical and the quantum regimes. 


\section{Concluding Remarks}

In the first part of this review on the fundamentals of the theory, we have given two routes to the establishment of stochastic gravity and derived a general (finite) expression for the noise kernel. In the second part, we gave three applications: the correlation functions of gravitons in a perturbed Minkowski metric, structure formation in stochastic-gravity theory and the outline of a program for the study of black-hole fluctuations and backreaction. We have also discussed the problem of the validity of semiclassical gravity, a central issue, which stochastic gravity is in a unique position to address.

We have pointed out a number of ongoing research projects related to the topics discussed in this review, such as the equivalence of the correlation functions to the metric perturbations obtained using the Einstein-Langevin equations and the quantum-correlation functions that follow from a pure quantum-field-theory calculation up to leading order in the large $N$ limit, the calculation of the spectrum of metric fluctuations in inflationary models driven by the trace anomaly due to conformally-coupled fields, the related problem of runaway solutions in backreaction equations and the issue of the coincidence limit in the noise kernel for black-hole fluctuations.

Theoretically, stochastic gravity is at the front line of the 'bottom-up' approach to quantum gravity $[185,187,188,190]$. Its pathway or angle starts from the well-defined and well-understood theory of semiclassical gravity. Structurally, as can be seen from the issues discussed and the applications given, stochastic gravity has a very rich constituency because it is based on quantum field theory and nonequilibrium statistical mechanics in a curved-spacetime context. The open systems concepts and the closed-time-path/influence-functional methods constitute an extended framework suitable for treating the backreaction and fluctuation problems of dynamical spacetimes interacting with quantum fields. We have seen applications to cosmological-structure formation and black-hole backreaction from particle creation. A more complete understanding of the backreaction of Hawking radiation in a fully-dynamical black-hole situation will enable one to address fundamental issues such as the black-hole end state and information-loss puzzles. The main reason why this program has not progressed as swiftly as desired is due more to technical rather than programatic difficulties (such as finding reasonable analytic approximations for the Green's function or the numerical evaluation of mode-sums near the black-hole horizon). Finally, the multiplex structure of this theory could be used to explore new lines of inquiry and launch new programs of research, such as nonequilibrium black-hole thermodynamics and the microscopic structures of spacetime. 


\section{Acknowledgements}

The materials presented here originated from the research of BLH with Antonio Campos, Nicholas Phillips, Alpan Raval, Albert Roura and Sukanya Sinha, and of EV with Rosario Martin and Albert Roura. We thank them as well as Daniel Arteaga, Andrew Matacz, Tom Shiokawa, and Yuhong Zhang for fruitful collaboration and their cordial friendship since their Ph.D. days. We enjoy lively discussions with our friends and colleagues Esteban Calzetta, Diego Mazzitelli and Juan Pablo Paz, whose work in the early years contributed towards the establishment of this field. We acknowledge useful discussions with Paul Anderson, Larry Ford, Ted Jacobson, Emil Mottola, Renaud Parentani, Raphael Sorkin and Richard Woodard. This work is supported in part by NSF grants PHY06-01550 and PHY05-51164, the MEC Research projects FPA-2004-04582C02 and FPA-2007-66665C02 and by DURSI 2005SGR00082. 


\section{References}

[1] Adler, S.L., Lieberman, J., and Ng, Y.J., "Regularization of the stress energy tensor for vector and scalar particles propagating in a general background metric", Ann. Phys. (N.Y.), 106, 279-321, (1977). 5

[2] Albrecht, A., and Steinhardt, P.J., "Cosmology for Grand Unified Theories with Radiatively Induced Symmetry Breaking", Phys. Rev. Lett., 48, 1220-1223, (1982). 2, 7

[3] Anderson, P.R., "Effects of quantum fields on singularities and particle horizons in the early universe", Phys. Rev. D, 28, 271-285, (1983). 1(b)ii, 2, 4

[4] Anderson, P.R., "Effects of quantum fields on singularities and particle horizons in the early universe. II", Phys. Rev. D, 29, 615-627, (1984). 1(b)ii, 2, 4

[5] Anderson, P.R., Binkley, M., Calderon, H., Hiscock, W.A., Mottola, E., and Vaulin, R., "Effects of quantized fields on the spacetime geometries of static spherically symmetric black holes", (2007). URL (cited on 22 January 2008):

http://arXiv.org/abs/arXiv:0709.4457. 1(b)ii

[6] Anderson, P.R., Hiscock, W.A., and Loranz, D.J., "Semiclassical stability of the extreme Reissner-Nordström black hole", Phys. Rev. Lett., 74, 4365-4368, (1995). Related online version (cited on 31 March 2003):

http://arXiv.org/abs/gr-qc/9504019. 8.1.1

[7] Anderson, P.R., Hiscock, W.A., and Samuel, D.A., "Stress energy tensor of quantized scalar fields in static black hole space-times", Phys. Rev. Lett., 70, 1739-1742, (1993). 8.1.1

[8] Anderson, P.R., Hiscock, W.A., and Samuel, D.A., "Stress-energy tensor of quantized scalar fields in static spherically symmetric space-times", Phys. Rev. D, 51, 4337-4358, (1995). 8.1.1, 8.3.1

[9] Anderson, P.R., Hiscock, W.A., Whitesell, J., and York Jr, J.W., "Semiclassical black hole in thermal equilibrium with a nonconformal scalar field", Phys. Rev. D, 50, 6427-6434, (1994). $8,8.2 .1$

[10] Anderson, P.R., Molina-Paris, C., and Mottola, E., "Linear response, validity of semiclassical gravity, and the stability of flat space", Phys. Rev. D, 67, 024026, 1-19, (2003). Related online version (cited on 31 March 2003):

http://arXiv.org/abs/gr-qc/0209075. 3a, 2.1, 3.1, 3.2, 3.3, 5, 6.5.1, 6.5.1, 8.1.2

[11] Anderson, P.R., Molina-Paris, C., and Mottola, E., "Linear response and the validity of the semi-classical approximation in gravity", (April 2004). URL (cited on 31 March 2003):

http://arXiv.org/abs/gr-qc/0204083. 2.1, 3.1, 3.2, 3.3, 5, 6.5.1, 8.1.2

[12] Anglin, J.R., Laflamme, R., Zurek, W.H., and Paz, J.P., "Decoherence, recoherence, and the black hole information paradox", Phys. Rev. D, 52, 2221-2231, (1995). Related online version (cited on 22 January 2008):

http://arXiv.org/abs/gr-qc/9411073. 8

[13] Ashtekar, A., "Large quantum gravity effects: Unforseen limitations of the classical theory", Phys. Rev. Lett., 77, 4864-4867, (1996). Related online version (cited on 22 January 2008): http://arXiv.org/abs/gr-qc/9610008. 3.3 
[14] Bakshi, P.M., and Mahanthappa, K.T., "Expectation value formalism in quantum field theory. 1", J. Math. Phys., 4, 1-11, (1963). 1, 1(b)iii, 4

[15] Balbinot, R., Fabbri, A., Fagnocchi, S., and Parentani, R., "Hawking radiation from acoustic black holes, short distance and back-reaction effects", Riv. Nuovo Cimento, 28(03), 1-55, (2005). Related online version (cited on 22 January 2008): http://arXiv.org/abs/gr-qc/0601079. 1(b)ii, 8

[16] Barceló, C., Liberati, S., and Visser, M., "Analogue Gravity", Living Rev. Relativity, 8, lrr2005-12, (2005). Related online version (cited on 22 January 2008): http://www. livingreviews .org/lrr-2005-12. 1(b)ii, 8

[17] Bardeen, J.M., "Gauge invariant cosmological perturbations", Phys. Rev. D, 22, 1882-1905, (1980). 7, 7.1

[18] Bardeen, J.M., "Black holes do evaporate thermally", Phys. Rev. Lett., 46, 382-385, (1981). $8,8.3,8.3 .1,8.3 .3$

[19] Bardeen, J.M., Steinhardt, P.J., and Turner, M.S., "Spontaneous Creation of Almost Scale - Free Density Perturbations in an Inflationary Universe", Phys. Rev. D, 28, 679, (1983). 7

[20] Barrabès, C., Frolov, V.P., and Parentani, R., "Metric fluctuation corrections to Hawking radiation", Phys. Rev. D, 59, 124010, 1-14, (1999). Related online version (cited on 31 March 2003):

http://arXiv.org/abs/gr-qc/9812076. 4b, 5a, 8.4

[21] Barrabès, C., Frolov, V.P., and Parentani, R., "Stochastically fluctuating black-hole geometry, Hawking radiation and the trans-Planckian problem", Phys. Rev. D, 62, 044020, 1-19, (2000). Related online version (cited on 31 March 2003):

http://arXiv.org/abs/gr-qc/0001102. 4b, 5a, 8.4

[22] Beetle, C., "Midi-superspace quantization of non-compact toroidally symmetric gravity", Adv. Theor. Math. Phys., 2, 471-495, (1998). Related online version (cited on 22 January 2008):

http://arXiv.org/abs/gr-qc/9801107. 3.3

[23] Bekenstein, J.D., "Black Holes and Entropy", Phys. Rev. D, 7, 2333-2346, (1973). 8

[24] Bekenstein, J.D., "Black hole fluctuations", in Christensen, S.M., ed., Quantum Theory of Gravity: Essays in Honor of the 60th Birthday of Bryce S. DeWitt, pp. 148-159, (Adam Hilger, Bristol, U.K., 1984). 8.3, 8.3.2, 8.3.2, 8.3.2, 8.3.3

[25] Bekenstein, J.D., "Do We Understand Black Hole Entropy?", in Jantzen, R.T., and Mac Keiser, G., eds., The Seventh Marcel Grossmann Meeting on Recent Developments in Theoretical and Experimental General Relativity, Gravitation and Relativistic Field Theories, Proceedings of the meeting held at Stanford University, 24-30 July 1994, pp. 39-58, (World Scientific, Singapore; River Edge, U.S.A., 1994). Related online version (cited on 31 March 2003):

http://arXiv.org/abs/gr-qc/9409015. 8

[26] Bekenstein, J.D., and Mukhanov, V.F., "Spectroscopy of the quantum black hole", Phys. Lett. B, 360, 7-12, (1995). Related online version (cited on 31 March 2003):

http://arXiv.org/abs/gr-qc/9505012. 8 
[27] Belinskii, V.A., Khalatnikov, I.M., and Lifshitz, E.M., "Oscillatory approach to a singular point in the relativistic cosmology", Adv. Phys., 19, 525-573, (1970). 2, 7

[28] Belinskii, V.A., Khalatnikov, I.M., and Lifshitz, E.M., "A general solution of the Einstein equations with a time singularity", Adv. Phys., 13, 639-667, (1982). 2

[29] Berger, B.K., "Quantum graviton creation in a model universe", Ann. Phys. (N.Y.), 83, 458-490, (1974). 2

[30] Berger, B.K., "Quantum cosmology: Exact solution for the Gowdy T3 model", Phys. Rev. D, 11, 2770-2780, (1975). 2

[31] Berger, B.K., "Scalar particle creation in an anisotropic universe", Phys. Rev. D, 12, 368$375,(1975) .2$

[32] Bernadotte, S., and Klinkhamer, F.R., "Bounds on length scales of spacetime foam models", Phys. Rev. D, 75, 024028, (2007). Related online version (cited on 22 January 2008):

http://arXiv.org/abs/hep-ph/0610216. 5b

[33] Bernard, W., and Callen, H.B., "Irreversible thermodynamics of nonlinear processes and noise in driven systems", Rev. Mod. Phys., 31, 1017-1044, (1959). 8.1.2

[34] Birrell, N.D., and Davies, P.C.W., Quantum fields in curved space, Cambridge Monographs on Mathematical Physics, (Cambridge University Press, Cambridge, U.K.; New York, U.S.A., 1982). 1b, 2, 3.1, 7.3, 8.3

[35] Borgman, J., and Ford, L.H., "Effects of quantum stress tensor fluctuations with compact extra dimensions", Phys. Rev. D, 70, 127701, (2004). Related online version (cited on 22 January 2008):

http://arXiv.org/abs/gr-qc/0406066. 3.3

[36] Borgman, J., and Ford, L.H., "The effects of stress tensor fluctuations upon focusing", Phys. Rev. D, 70, 064032, (2004). Related online version (cited on 22 January 2008):

http://arXiv.org/abs/gr-qc/0307043. 3.3

[37] Borgman, J., and Ford, L.H., "Stochastic gravity and the Langevin-Raychaudhuri equation", Int. J. Mod. Phys. A, 20, 2364-2373, (2005). 3.3

[38] Boyanovsky, D., de Vega, H.J., Holman, R., Lee, D.S., and Singh, A., "Dissipation via particle production in scalar field theories", Phys. Rev. D, 51, 4419-4444, (1995). Related online version (cited on 31 March 2003):

http://arXiv.org/abs/hep-ph/9408214. 4.2

[39] Brandt, F.T., and Frenkel, J., "The structure of the graviton self-energy at finite temperature", Phys. Rev. D, 58, 085012, 1-11, (1998). Related online version (cited on 31 March 2003):

http://arXiv.org/abs/hep-th/9803155. 8.2, 8.2.3, 8.2.3

[40] Braunstein, S.L., and Pati, A.K., "Quantum information cannot be completely hidden in correlations: Implications for the black-hole information paradox", Phys. Rev. Lett., 98, 080502, (2007). Related online version (cited on 22 January 2008):

http://arXiv.org/abs/gr-qc/0603046. 8

[41] Brown, M.R., and Ottewill, A.C., "Effective actions and conformal transformations", Phys. Rev. D, 31, 2514-2520, (1985). 8.1.1 
[42] Brown, M.R., Ottewill, A.C., and Page, D.N., "Conformally invariant quantum field theory in static Einstein space-times", Phys. Rev. D, 33, 2840-2850, (1986). 8.1.1

[43] Brun, T.A., "Quasiclassical equations of motion for nonlinear Brownian systems", Phys. Rev. D, 47, 3383-3393, (1993). Related online version (cited on 31 March 2003): http://arXiv.org/abs/gr-qc/9306013. 1(c)iii, 2.1

[44] Bunch, T.S., "On the renormalization of the quantum stress tensor in curved space-time by dimensional regularization", J. Phys. A, 12, 517-531, (1979). 3.1

[45] Caldeira, A.O., and Leggett, A.J., "Path integral approach to quantum Brownian motion", Physica A, 121, 587-616, (1983). 8.2.3, 8.2.4, 8.2.6

[46] Caldeira, A.O., and Leggett, A.J., "Influence of damping on quantum interference: An exactly soluble model", Phys. Rev. A, 31, 1059-1066, (1985). 1(c)iii, 2.1, 4

[47] Callan Jr, C.G., Giddings, S.B., Harvey, J.A., and Strominger, A., "Evanescent black holes", Phys. Rev. D, 45, 1005-1009, (1992). Related online version (cited on 22 January 2008): http://arXiv.org/abs/hep-th/9111056. 8

[48] Callen, H.B., and Greene, R.F., "On a theorem of irreversible thermodynamics", Phys. Rev., 86, 702-710, (1952). 8.1.2

[49] Callen, H.B., and Welton, T.A., "Irreversibility and generalized noise", Phys. Rev., 83, 3440, (1951). 8.1.2

[50] Calzetta, E., "Memory loss and asymptotic behavior in minisuperspace cosmological models", Class. Quantum Grav., 6, L227-L231, (1989). 1d, 4

[51] Calzetta, E., "Anisotropy dissipation in quantum cosmology", Phys. Rev. D, 43, 2498-2509, (1991). 1d, 4

[52] Calzetta, E.A., Campos, A., and Verdaguer, E., "Stochastic semiclassical cosmological models", Phys. Rev. D, 56, 2163-2172, (1997). Related online version (cited on 31 March 2003): http://arXiv.org/abs/gr-qc/9704010. 2c, 2.1, 6

[53] Calzetta, E.A., and Gonorazky, S., "Primordial fluctuations from nonlinear couplings", Phys. Rev. D, 55, 1812-1821, (1997). Related online version (cited on 31 March 2003): http://arXiv.org/abs/gr-qc/9608057. 4d, 7.3

[54] Calzetta, E.A., and Hu, B.L., "Closed time path functional formalism in curved space-time: application to cosmological backreaction problems", Phys. Rev. D, 35, 495-509, (1987). 1, 1(b)iii, 2a, 4

[55] Calzetta, E.A., and Hu, B.L., "Nonequilibrium quantum fields: closed time path effective action Wigner function and Boltzmann equation", Phys. Rev. D, 37, 2878-2900, (1988). 1

[56] Calzetta, E.A., and Hu, B.L., "Dissipation of quantum fields from particle creation", Phys. Rev. D, 40, 656-659, (1989). 1, 1(b)iii, 4

[57] Calzetta, E.A., and Hu, B.L., "Decoherence of Correlation Histories", in Hu, B.L., and Jacobson, T.A., eds., Directions in General Relativity, Vol. 2, Proceedings of the 1993 International Symposium, Maryland: Papers in honor of Dieter Brill, pp. 38-65, (Cambridge University Press, Cambridge, U.K.; New York, U.S.A., 1993). Related online version (cited on 3 May 2005):

http://arxiv.org/abs/gr-qc/9302013. 1, 6(c)i 
[58] Calzetta, E.A., and Hu, B.L., "Noise and fluctuations in semiclassical gravity", Phys. Rev. $D$, 49, 6636-6655, (1994). Related online version (cited on 31 March 2003): http://arXiv.org/abs/gr-qc/9312036. 2b, 2c, 2.1, 4, 4.2, 5, 5.2.3, 8.2.4

[59] Calzetta, E.A., and Hu, B.L., "Correlations, Decoherence, Dissipation, and Noise in Quantum Field Theory", in Fulling, S.A., ed., Heat Kernel Techniques and Quantum Gravity, Discourses in Mathematics and Its Applications, vol. 4, pp. 261-302, (Texas A\&M University, College Station, U.S.A., 1995). 1

[60] Calzetta, E.A., and Hu, B.L., "Quantum fluctuations, decoherence of the mean field, and structure formation in the early universe", Phys. Rev. D, 52, 6770-6788, (1995). Related online version (cited on 31 March 2003):

http://arXiv.org/abs/gr-qc/9505046. 4d, 7.3

[61] Calzetta, E.A., and Hu, B.L., "Stochastic dynamics of correlations in quantum field theory: From Schwinger-Dyson to Boltzmann-Langevin equation", Phys. Rev. D, 61, 025012, 1-22, (2000). Related online version (cited on 31 March 2003):

http://arXiv.org/abs/hep-ph/9903291. 1, 6(c)i, 3.2

[62] Calzetta, E.A., and Hu, B.L., Nonequilibrium Quantum Field Theory, (Cambridge University Press, Cambridge, England, U.K., 2008). 1c

[63] Calzetta, E.A., and Kandus, A., "Spherically symmetric nonlinear structures", Phys. Rev. $D, \mathbf{5 5}, 1795-1811,(1997)$. Related online version (cited on 31 March 2003):

http://arXiv.org/abs/astro-ph/9603125. 4.1

[64] Calzetta, E.A., Roura, A., and Verdaguer, E., "Vacuum decay in quantum field theory", Phys. Rev. D, 64, 105008, 1-21, (2001). Related online version (cited on 31 March 2003): http://arXiv.org/abs/hep-ph/0106091. 3.2

[65] Calzetta, E.A., Roura, A., and Verdaguer, E., "Dissipation, Noise, and Vacuum Decay in Quantum Field Theory", Phys. Rev. Lett., 88, 010403, 1-4, (2002). Related online version (cited on 31 March 2003):

http://arXiv.org/abs/hep-ph/0101052. 3.2

[66] Calzetta, E.A., Roura, A., and Verdaguer, E., "Stochastic description for open quantum systems", Physica A, 319, 188-212, (2003). Related online version (cited on 31 March 2003): http://arXiv.org/abs/quant-ph/0011097. 3.2, 3.2, 7.4

[67] Calzetta, E.A., and Verdaguer, E., "Noise induced transitions in semiclassical cosmology", Phys. Rev. D, 59, 083513, 1-24, (1999). Related online version (cited on 31 March 2003): http://arXiv.org/abs/gr-qc/9807024. 4c

[68] Camporesi, R., "Harmonic analysis and propagators on homogeneous spaces", Phys. Rep., 196, 1-134, (1990). 5

[69] Campos, A., and Hu, B.L., "Nonequilibrium dynamics of a thermal plasma in a gravitational field", Phys. Rev. D, 58, 125021, 1-15, (1998). Related online version (cited on 31 March 2003):

http://arXiv.org/abs/hep-ph/9805485. 2a, 4e, 4, 8.1.3, 8.2, 8.2.2, 8.2.3, 8.2.3, 8.2.3, 8.2.3, 8.2.5, 8.2.5, 8.2.6, 8.3.2

[70] Campos, A., and Hu, B.L., "Fluctuations in a thermal field and dissipation of a black hole spacetime: Far-field limit", Int. J. Theor. Phys., 38, 1253-1271, (1999). Related online 
version (cited on 31 March 2003):

http://arXiv.org/abs/gr-qc/9812034. 4e, 4, 8.1.3, 8.2, 8.2.2, 8.2.3, 8.2.3, 8.2.3, 8.2.3, $8.2 .5,8.2 .5,8.2 .6,8.3 .2$

[71] Campos, A., Martín, R., and Verdaguer, E., "Back reaction in the formation of a straight cosmic string", Phys. Rev. D, 52, 4319-4336, (1995). Related online version (cited on 31 March 2003):

http://arXiv.org/abs/gr-qc/9505003. 6.3

[72] Campos, A., and Verdaguer, E., "Semiclassical equations for weakly inhomogeneous cosmologies", Phys. Rev. D, 49, 1861-1880, (1994). Related online version (cited on 31 March 2003): http://arXiv.org/abs/gr-qc/9307027. 2a, 4, 6.3

[73] Campos, A., and Verdaguer, E., "Stochastic semiclassical equations for weakly inhomogeneous cosmologies", Phys. Rev. D, 53, 1927-1937, (1996). Related online version (cited on 31 March 2003):

http://arXiv.org/abs/gr-qc/9511078. 2c, 2.1, 4, 4.2, 5, 5.2.3, 6, 6.3, 7.4, 8.1.2, 8.3.2

[74] Campos, A., and Verdaguer, E., "Back-reaction equations for isotropic cosmologies when nonconformal particles are created", Int. J. Theor. Phys., 36, 2525-2543, (1997). 2c, 2.1, 6

[75] Candelas, P., "Vacuum Polarization in Schwarzschild Space-Time", Phys. Rev. D, 21, 21852202, (1980). 8.3.1

[76] Candelas, P., and Sciama, D.W., "Irreversible thermodynamics of black holes", Phys. Rev. Lett., 38, 1372-1375, (1977). 2a, 4e, 8.1.2, 8.2.6

[77] Capper, D.M., and Duff, M.J., "Trace anomalies in dimensional regularization", Nuovo Cimento A, 23, 173-183, (1974). 5.2 .3

[78] Carlip, S., "Spacetime Foam and the Cosmological Constant", Phys. Rev. Lett., 79, 40714074, (1997). Related online version (cited on 31 March 2003):

http://arXiv.org/abs/gr-qc/9708026. 5b

[79] Carlip, S., "Dominant topologies in Euclidean quantum gravity", Class. Quantum Grav., 15, 2629-2638, (1998). Related online version (cited on 31 March 2003):

http://arXiv.org/abs/gr-qc/9710114. 5b

[80] Casher, A., Englert, F., Itzhaki, N., Massar, S., and Parentani, R., "Black hole horizon fluctuations", Nucl. Phys. B, 484, 419-434, (1997). Related online version (cited on 31 March 2003):

http://arXiv.org/abs/hep-th/9606106. 8.3, 8.3.2

[81] Cespedes, J., and Verdaguer, E., "Particle production in inhomogeneous cosmologies", Phys. Rev. D, 41, 1022-1033, (1990). 2

[82] Chou, K., Su, Z., Hao, B., and Yu, L., "Equilibrium and non equilibrium formalisms made unified", Phys. Rep., 118, 1-131, (1985). 1, 1(b)iii, 4

[83] Christensen, S.M., "Vacuum expectation value of the stress tensor in an arbitrary curved background: The covariant point separation method", Phys. Rev. D, 14, 2490-2501, (1976). $2,3.1,3.1,5.1,5.1 .1$

[84] Christensen, S.M., "Regularization, renormalization, and covariant geodesic point separation", Phys. Rev. D, 17, 946-963, (1978). 2, 3.1, 3.1, 5.1, 5.1.1 
[85] Christiansen, W.A., Ng, Y.J., and van Dam, H., "Probing spacetime foam with extragalactic sources", Phys. Rev. Lett., 96, 051301, (2006). Related online version (cited on 22 January 2008):

http://arXiv.org/abs/gr-qc/0508121.5b

[86] Cognola, G., Elizalde, E., and Zerbini, S., "Fluctuations of quantum fields via zeta function regularization", Phys. Rev. D, 65, 085031, 1-8, (2002). Related online version (cited on 31 March 2003):

http://arXiv.org/abs/hep-th/0201152. 2.1, 3.1, 5

[87] Cooper, F., Habib, S., Kluger, Y., Mottola, E., Paz, J.P., and Anderson, P.R., "Nonequilibrium quantum fields in the large- $N$ expansion", Phys. Rev. D, 50, 2848-2869, (1994). Related online version (cited on 31 March 2003): http://arXiv.org/abs/hep-ph/9405352. 1, 1(b)iii, 4

[88] Davies, E.B., Quantum Theory of Open Systems, (Academic Press, London, U.K.; New York, U.S.A., 1976). 1, 1(c)i

[89] de Almeida, A.P., Brandt, F.T., and Frenkel, J., "Thermal matter and radiation in a gravitational field", Phys. Rev. D, 49, 4196-4208, (1994). Related online version (cited on 31 March 2003):

http://arXiv.org/abs/hep-th/9309098. 8.2, 8.2.3, 8.2.3

[90] Décanini, Y., and Folacci, A., "Off-diagonal coefficients of the DeWitt-Schwinger and Hadamard representations of the Feynman propagator", Phys. Rev. D, 73, 044027, 1-38, (2006). Related online version (cited on 15 April 2008):

http://arXiv.org/abs/gr-qc/0511115. 5.1.1

[91] Deser, S., "Plane waves do not polarize the vacuum", J. Phys. A, 8, 1972-1974, (1975). 6.1

[92] DeWitt, B.S., Dynamical Theory of Groups and Fields, (Gordon and Breach, New York, U.S.A., 1965). 5.1

[93] DeWitt, B.S., "Quantum field theory in curved space-time", Phys. Rep., 19, 295-357, (1975). $2,5.1$

[94] DeWitt, B.S., "Effective action for expectation values", in Penrose, R., and Isham, C.J., eds., Quantum concepts in space and time, (Clarendon Press; Oxford University Press, Oxford, U.K.; New York, U.S.A., 1986). 1, 1(b)iii, 2a, 4

[95] Donoghue, J.F., "General relativity as an effective field theory: The leading quantum corrections", Phys. Rev. D, 50, 3874-3888, (1994). Related online version (cited on 31 March 2003):

http://arXiv.org/abs/gr-qc/9405057. 4, 4.1

[96] Donoghue, J.F., "Leading quantum correction to the Newtonian potential", Phys. Rev. Lett., 72, 2996-2999, (1994). Related online version (cited on 31 March 2003):

http://arXiv.org/abs/gr-qc/9310024. 4, 4.1

[97] Donoghue, J.F., "The quantum theory of general relativity at low energies", Helv. Phys. Acta, 69, 269-275, (1996). Related online version (cited on 31 March 2003):

http://arXiv.org/abs/gr-qc/9607039. 4, 4.1 
[98] Donoghue, J.F., "Introduction to the Effective Field Theory Description of Gravity", in Cornet, F., and Herrero, M.J., eds., Advanced School on Effective Theories, Proceedings of the conference held in Almuñecar, Granada, Spain, 26 June-1 July 1995, pp. 217-240, (World Scientific, Singapore, 1997). Related online version (cited on 31 March 2003): http://arXiv.org/abs/gr-qc/9512024. 4, 4.1

[99] Dowker, F., and Kent, A., "Properties of consistent histories", Phys. Rev. Lett., 75, 30383041, (1995). Related online version (cited on 31 March 2003): http://arXiv.org/abs/gr-qc/9409037. 1(c)iii, 2.1

[100] Dowker, F., and Kent, A., "On the consistent histories approach to quantum mechanics", $J$. Stat. Phys., 82, 1575-1646, (1996). Related online version (cited on 31 March 2003): http://arXiv.org/abs/gr-qc/9412067. 1(c)iii, 2.1

[101] Dowker, H.F., and Halliwell, J.J., "The Quantum mechanics of history: The Decoherence functional in quantum mechanics", Phys. Rev. D, 46, 1580-1609, (1992). 1(c)iii, 2.1, 3.2

[102] Duff, M.J., "Covariant Quantization of Gravity", in Isham, C.J., Penrose, R., and Sciama, D.W., eds., Quantum Gravity: An Oxford Symposium, Symposium held at the Rutherford Laboratory on February 15-16, 1974, (Clarendon Press, Oxford, U.K., 1975). 5.2.3

[103] Einstein, A., "Über die von der molekularkinetischen Theorie der Wärme geforderte Bewegung von in ruhenden Flüssigkeiten suspendierten Teilchen", Ann. Phys. (Leipzig), 17, 549-560, (1905). 8.1.2

[104] Einstein, A., "Zur Theorie der Brownschen Bewegung", Ann. Phys. (Leipzig), 19, 371-381, (1906). 8.1.2

[105] Eling, C., Guedens, R., and Jacobson, T.A., "Nonequilibrium Thermodynamics of Spacetime", Phys. Rev. Lett., 96, 121301, (2006). Related online version (cited on 22 January 2008):

http://arXiv.org/abs/gr-qc/0602001.6a, 8

[106] Elizalde, E., Odintsov, S.D., Romeo, A., Bytsenko, A.A., and Zerbini, S., Zeta Regularization Techniques with Applications, (World Scientific, Singapore; River Edge, U.S.A., 1994). 5

[107] Feynman, R.P., and Hibbs, A.R., Quantum Mechanics and Path Integrals, International Series in Pure and Applied Physics, (McGraw-Hill, New York, U.S.A., 1965). 1, 1(c)ii, 5.2.3

[108] Feynman, R.P., and Vernon Jr, F.L., "The theory of a general quantum system interacting with a linear dissipative system", Ann. Phys. (N.Y.), 24, 118-173, (1963). 1, 1(c)ii, 2.1, 4, 5.2 .3

[109] Fischetti, M.V., Hartle, J.B., and Hu, B.L., "Quantum fields in the early universe. I. Influence of trace anomalies on homogeneous, isotropic, classical geometries", Phys. Rev. D, 20, 17571771, (1979). 1(b)ii, 2, 4

[110] Flanagan, É.É., and Wald, R.M., "Does back reaction enforce the averaged null energy condition in semiclassical gravity?", Phys. Rev. D, 54, 6233-6283, (1996). Related online version (cited on 31 March 2003):

http://arXiv.org/abs/gr-qc/9602052. 3.3, 6.1, 6.3, 6.4, 6.4.1, 6.4.1, 6.5.1, 6.5.1, 6.5.1, 6.5.3, 8.3

[111] Ford, L.H., "Gravitational radiation by quantum systems", Ann. Phys. (N.Y.), 144, 238-248, (1982). 3a, 2.1, 3.1, 3.3 
[112] Ford, L.H., "Spacetime metric and lightcone fluctuations", Int. J. Theor. Phys., 38, 29412958, (1999). 3.3

[113] Ford, L.H., "Stress tensor fluctuations and stochastic space-times", Int. J. Theor. Phys., 39, 1803-1815, (2000). 2.1, 3.1, 5

[114] Ford, L.H., and Svaiter, N.F., "Cosmological and black hole horizon fluctuations", Phys. Rev. D, 56, 2226-2235, (1997). Related online version (cited on 31 March 2003):

http://arXiv.org/abs/gr-qc/9704050. 4b, 3.3, 8.3, 8.4

[115] Ford, L.H., and Wu, C.-H., "Stress Tensor Fluctuations and Passive Quantum Gravity", Int. J. Theor. Phys., 42, 15-26, (2003). Related online version (cited on 31 March 2003): http://arXiv.org/abs/gr-qc/0102063. 2.1, 3.1, 3.3, 5, 8.4

[116] Freidel, L., and Krasnov, K., "A New Spin Foam Model for 4d Gravity", (2007). URL (cited on 22 January 2008):

http://arXiv.org/abs/arXiv:0708.1595. 5b

[117] Frieman, J.A., "Particle creation in inhomogeneous spacetimes", Phys. Rev. D, 39, 389-398, (1989). 2

[118] Frolov, V.P., and Zel'nikov, A.I., "Vacuum polarization by a massive scalar field in Schwarzschild space-time", Phys. Lett. B, 115, 372-374, (1982). 8.1.1

[119] Frolov, V.P., and Zel'nikov, A.I., "Vacuum polarization of massive fields near rotating black holes", Phys. Rev. D, 29, 1057-1066, (1984). 8.1.1

[120] Frolov, V.P., and Zel'nikov, A.I., "Killing approximation for vacuum and thermal stressenergy tensor in static space-times", Phys. Rev., D35, 3031-3044, (1987). 8.1.1

[121] Fulling, S.A., Aspects of Quantum Field Theory in Curved Space-Time, (Cambridge University Press, Cambridge, U.K.; New York, U.S.A., 1989). 1b, 2, 3.1, 3.1, 5.1.1, 6.1

[122] Garay, L.J., "Spacetime foam as a quantum thermal bath", Phys. Rev. Lett., 80, 2508-2511, (1998). Related online version (cited on 31 March 2003):

http://arXiv.org/abs/gr-qc/9801024. 5b

[123] Garay, L.J., "Thermal properties of spacetime foam", Phys. Rev. D, 58, 124015, 1-11, (1998). Related online version (cited on 31 March 2003):

http://arXiv.org/abs/gr-qc/9806047. 5b

[124] Garay, L.J., "Quantum evolution in spacetime foam", Int. J. Mod. Phys. A, 14, 4079-4120, (1999). Related online version (cited on 31 March 2003):

http://arXiv.org/abs/gr-qc/9911002. 5b

[125] Garriga, J., and Verdaguer, E., "Scattering of quantum particles by gravitational plane waves", Phys. Rev. D, 43, 391-401, (1991). 6.1

[126] Gell-Mann, M., and Hartle, J.B., "Quantum mechanics in the light of quantum cosmology", in Zurek, W.H., ed., Complexity, Entropy and the Physics of Information, Proceedings of the workshop, held May-June, 1989, in Santa Fe, New Mexico, Santa Fe Institute Studies in the Sciences of Complexity, vol. 8, pp. 425-458, (Addison-Wesley, Redwood City, U.S.A., 1990). 1(c)iii, 2.1 
[127] Gell-Mann, M., and Hartle, J.B., "Classical equations for quantum systems", Phys. Rev. D, 47, 3345-3382, (1993). Related online version (cited on 31 March 2003): http://arXiv.org/abs/gr-qc/9210010. 2.1, 3.2

[128] Gibbons, G.W., "Quantized fields propagating in plane wave spacetimes", Commun. Math. Phys., 45, 191-202, (1975). 6.1

[129] Gibbons, G.W., and Perry, M.J., "Black holes and thermal Green functions", Proc. R. Soc. London, Ser. A, 358, 467-494, (1978). 8.2

[130] Giddings, S.B., "(Non)perturbative gravity, nonlocality, and nice slices", Phys. Rev. D, 74, 106009, (2006). Related online version (cited on 22 January 2008): http://arXiv.org/abs/hep-th/0606146. 8

[131] Giulini, D., Joos, E., Kiefer, C., Kupsch, J., Stamatescu, I.O., Zeh, H.D., Stamatescu, I.-O., and Zeh, H.-D., Decoherence and the Appearance of a Classical World in Quantum Theory, (Springer, Berlin, Germany; New York, U.S.A., 1996). 1(c)iii, 2.1, 4

[132] Gleiser, M., and Ramos, R.O., "Microphysical approach to nonequilibrium dynamics of quantum fields", Phys. Rev. D, 50, 2441-2455, (1994). Related online version (cited on 31 March 2003):

http://arXiv.org/abs/hep-ph/9311278. 4.2

[133] Grabert, H., Schramm, P., and Ingold, G.L., "Quantum Brownian motion: the functional integral approach", Phys. Rep., 168, 115-207, (1988). 8.2.3, 8.2.4, 8.2.6

[134] Greiner, C., and Müller, B., "Classical Fields Near Thermal Equilibrium", Phys. Rev. D, 55, 1026-1046, (1997). Related online version (cited on 31 March 2003):

http://arXiv.org/abs/hep-th/9605048. 4

[135] Grib, A.A., Mamayev, S.G., and Mostepanenko, V.M., Vacuum quantum effects in strong fields, (Friedmann Laboratory Publishing, St. Petersburg, Russia, 1994). 1b, 2, 6.1

[136] Griffiths, R.B., "Consistent histories and the interpretation of quantum mechanics", J. Stat. Phys., 36, 219-272, (1984). 1(c)iii, 2.1

[137] Grishchuk, L.P., "Graviton creation in the early universe", Ann. N.Y. Acad. Sci., 302, 439444, (1976). 1(b)ii, 2, 4

[138] Gross, D.J., Perry, M.J., and Yaffe, L.G., "Instability of flat space at finite temperature", Phys. Rev. D, 25, 330-355, (1982). 8.2, 8.2.3

[139] Gu, Z.-C., and Wen, X.-G., "A lattice bosonic model as a quantum theory of gravity", (2006). URL (cited on 22 January 2008):

http://arXiv.org/abs/gr-qc/0606100. 6a, 8

[140] Guth, A.H., "The inflationary universe: A possible solution to the horizon and flatness problems", Phys. Rev. D, 23, 347-356, (1981). 2, 7

[141] Guth, A.H., and Pi, S.Y., "Fluctuations in the New Inflationary Universe", Phys. Rev. Lett., 49, 1110-1113, (1982). 7

[142] Hájíček, P., and Israel, W., "What, no black hole evaporation?", Phys. Lett. A, 80, 9-10, (1980). 8 
[143] Halliwell, J.J., "Decoherence in quantum cosmology", Phys. Rev. D, 39, 2912-2923, (1989). $1 \mathrm{~d}, 4$

[144] Halliwell, J.J., "Quantum mechanical histories and the uncertainty principle. 2. Fluctuations about classical predictability", Phys. Rev. D, 48, 4785-4799, (1993). Related online version (cited on 31 March 2003):

http://arXiv.org/abs/gr-qc/9307013. 1(c)iii, 2.1, 3.2

[145] Halliwell, J.J., "A Review of the decoherent histories approach to quantum mechanics", Ann. N.Y. Acad. Sci., 755, 726-740, (1995). Related online version (cited on 31 March 2003): http://arXiv.org/abs/gr-qc/9407040. 1(c)iii, 2.1

[146] Halliwell, J.J., "Effective theories of coupled classical and quantum variables from decoherent histories: A new approach to the backreaction problem", Phys. Rev. D, 57, 2337-2348, (1998). Related online version (cited on 31 March 2003):

http://arXiv.org/abs/quant-ph/9705005. 1(c)iii, 2.1, 3.2

[147] Hartle, J.B., "Effective potential approach to graviton production in the early universe", Phys. Rev. Lett., 39, 1373-1376, (1977). 1(b)ii, 2, 4

[148] Hartle, J.B., "Quantum effects in the early universe. 5. Finite particle production without trace anomalies", Phys. Rev. D, 23, 2121-2128, (1981). 1(b)ii, 2, 4

[149] Hartle, J.B., "The Quantum Mechanics of Closed Systems", in Hu, B.L., Ryan Jr, M.P., and Vishveswara, C.V., eds., Directions in General Relativity, Vol. 1, Proceedings of the 1993 International Symposium, Maryland: Papers in honor of Charles Misner, pp. 104-124, (Cambridge University Press, Cambridge, U.K.; New York, U.S.A., 1993). 1(c)iii, 2.1

[150] Hartle, J.B., "Spacetime quantum mechanics and the quantum mechanics of spacetime", in Julia, B., and Zinn-Justin, J., eds., Gravitation and Quantizations, Proceedings of the Les Houches Summer School, Session LVII, 5 July-1 August 1992, (Elsevier, Amsterdam, Netherlands, New York, U.S.A., 1995). 3.2

[151] Hartle, J.B., and Hawking, S.W., "Path-integral derivation of black-hole radiance", Phys. Rev. D, 13, 2188-2203, (1976). 8.2

[152] Hartle, J.B., and Horowitz, G.T., "Ground state expectation value of the metric in the $1 / N$ or semiclassical approximation to quantum gravity", Phys. Rev. D, 24, 257-274, (1981). 3.1, 3.3.1, 6.4.1

[153] Hartle, J.B., and Hu, B.L., "Quantum effects in the early universe. II. Effective action for scalar fields in homogeneous cosmologies with small anisotropy", Phys. Rev. D, 20, 1772 1782, (1979). 1(b)ii, 2, 4

[154] Hartle, J.B., and Hu, B.L., "Quantum effects in the early universe. III. Dissipation of anisotropy by scalar particle production", Phys. Rev. D, 21, 2756-2769, (1980). 1(b)ii, 2,4

[155] Hawking, S.W., "Black hole explosions?", Nature, 248, 30-31, (1974). 2

[156] Hawking, S.W., "Particle creation by black holes", Commun. Math. Phys., 43, 199-220, (1975). 2, 8

[157] Hawking, S.W., "Black Holes and Thermodynamics", Phys. Rev. D, 13, 191-197, (1976). 8 
[158] Hawking, S.W., "Breakdown of Predictability in Gravitational Collapse", Phys. Rev. D, 14, 2460-2473, (1976). 8

[159] Hawking, S.W., "The Development of Irregularities in a Single Bubble Inflationary Universe", Phys. Lett. B, 115, 295, (1982). 7

[160] Hawking, S.W., "Information loss in black holes", Phys. Rev. D, 72, 084013, (2005). Related online version (cited on 22 January 2008):

http://arXiv.org/abs/hep-th/0507171. 8

[161] Hawking, S.W., and Hertog, T., "Living with ghosts", Phys. Rev. D, 65, 103515, (2002). Related online version (cited on 22 January 2008):

http://arXiv.org/abs/hep-th/0107088. 6.5.1, 6.5.2, 6.5.4

[162] Hawking, S.W., Hertog, T., and Reall, H.S., "Trace anomaly driven inflation", Phys. Rev. $D$, 63, 083504, 1-23, (2001). Related online version (cited on 31 March 2003):

http://arXiv .org/abs/hep-th/0010232. 4d, 6.3, 6.5.1, 6.5.2, 6.5.3, 6.5.4, 7.4

[163] Hawking, S.W., and Page, D.N., "Thermodynamics of Black Holes in Anti-de Sitter Space", Commun. Math. Phys., 87, 577-588, (1983). 8.2

[164] Herzog, C.P., "The hydrodynamics of M-theory", J. High Energy Phys., 2002(12), 026, (2002). Related online version (cited on 22 January 2008):

http://arXiv.org/abs/hep-th/0210126. 6a, 8

[165] Hiscock, W.A., Larson, S.L., and Anderson, P.R., "Semiclassical effects in black hole interiors", Phys. Rev. D, 56, 3571-3581, (1997). Related online version (cited on 31 March 2003): http://arXiv.org/abs/gr-qc/9701004.1(b)ii, 8.1, 8.1.1

[166] Hochberg, D., and Kephart, T.W., "Gauge field back reaction on a black hole", Phys. Rev. D, 47, 1465-1470, (1993). Related online version (cited on 31 March 2003):

http://arXiv.org/abs/gr-qc/9211008. 8, 8.2.1

[167] Hochberg, D., Kephart, T.W., and York Jr, J.W., "Positivity of entropy in the semiclassical theory of black holes and radiation", Phys. Rev. D, 48, 479-484, (1993). Related online version (cited on 31 March 2003):

http://arXiv.org/abs/gr-qc/9211009. 8, 8.2.1

[168] Holzhey, C.F.E., and Wilczek, F., "Black holes as elementary particles", Nucl. Phys. B, 380, 447-477, (1992). Related online version (cited on 22 January 2008):

http://arXiv.org/abs/hep-th/9202014. 8

[169] Horowitz, G.T., "Semiclassical relativity: The weak field limit", Phys. Rev. D, 21, 1445-1461, (1980). 3a, 2.1, 3.3, 6.3, 6.3, 6.4, 6.4.1, 6.4.1, 6.5.1

[170] Horowitz, G.T., "Is flat space-time unstable?", in Isham, C.J., Penrose, R., and Sciama, D.W., eds., Quantum Gravity 2: A Second Oxford Symposium, Proceedings of the Second Oxford Symposium on Quantum Gravity, held in April 1980 in Oxford, pp. 106-130, (Clarendon Press; Oxford University Press, Oxford, U.K.; New York, U.S.A., 1981). 3a, 3.3, 6.3, 6.4 .1

[171] Horowitz, G.T., "The Origin of Black Hole Entropy in String Theory", in Cho, Y.M., Lee, C.H., and Kim, S.-W., eds., Gravitation and Cosmology, Proceedings of the Pacific Conference, February 1-6, 1996, Sheraton Walker-Hill, Seoul, Korea, pp. 46-63, (World Scientific, Singapore; River Edge, U.S.A., 1999). Related online version (cited on 31 March 2003):

http://arXiv.org/abs/gr-qc/9604051. 8 
[172] Horowitz, G.T., and Polchinski, J., "A correspondence principle for black holes and strings", Phys. Rev. D, 55, 6189-6197, (1997). Related online version (cited on 31 March 2003): http://arXiv.org/abs/hep-th/9612146. 8

[173] Horowitz, G.T., and Polchinski, J., "Gauge/gravity duality", (2006). URL (cited on 22 January 2008): http://arXiv.org/abs/gr-qc/0602037. 6a, 8

[174] Horowitz, G.T., and Wald, R.M., "Dynamics of Einstein's equations modified by a higher order derivative term", Phys. Rev. D, 17, 414-416, (1978). 6.4.1

[175] Horowitz, G.T., and Wald, R.M., "Quantum stress energy in nearly conformally flat spacetimes", Phys. Rev. D, 21, 1462-1465, (1980). 3.1

[176] Horowitz, G.T., and Wald, R.M., "Quantum stress energy in nearly conformally flat spacetimes. II. Correction of formula", Phys. Rev. D, 25, 3408-3409, (1982). 3.1

[177] Howard, K.W., "Vacuum in Schwarzschild space-time", Phys. Rev. D, 30, 2532-2547, (1984). 8.1.1, 8.3.1

[178] Howard, K.W., and Candelas, P., "Quantum stress tensor in Schwarzschild space-time", Phys. Rev. Lett., 53, 403-406, (1984). 8.1.1, 8.3.1

[179] Hu, B.L., "Scalar waves in the mixmaster universe. II. Particle creation", Phys. Rev. D, 9, 3263-3281, (1974). 2

[180] Hu, B.L., "Effect of finite temperature quantum fields on the early universe", Phys. Lett. B, 103, 331-337, (1981). 8.2

[181] Hu, B.L., "Disspation in quantum fields and semiclassical gravity", Physica A, 158, 399-424, (1989). 1, 2b, 2.1, 4

[182] Hu, B.L., "Quantum and statistical effects in superspace cosmology", in Audretsch, J., and De Sabbata, V., eds., Quantum Mechanics in Curved Space-Time, Proceedings of a NATO Advanced Research Workshop, held May 2-12, 1989, in Erice, Sicily, Italy, NATO ASI Series B, vol. 230, (Plenum Press, New York, U.S.A., 1990). 1d, 4

[183] Hu, B.L., "Quantum statistical fields in gravitation and cosmology", in Kobes, R., and Kunstatter, G., eds., Third International Workshop on Thermal Field Theory and Applications, CNRS Summer Institute, Banff, 1993, (World Scientific, Singapore, 1994). 2b, 8.1.1, 8.2.6

[184] Hu, B.L., "Correlation dynamics of quantum fields and black hole information paradox", in Sánchez, N., and Zichichi, A., eds., String Gravity and Physics at the Planck Energy Scale, Proceedings of the NATO Advanced Study Institute, Erice, Italy, 8-19,September, 1995, NATO ASI Series C, vol. 476, (Kluwer, Dordrecht, Netherlands; Boston, U.S.A., 1996). 8

[185] Hu, B.L., "General Relativity as Geometro-Hydrodynamics", (July 1996). URL (cited on 31 March 2003):

http://arXiv.org/abs/gr-qc/9607070. 6a, 5, 8, 9

[186] Hu, B.L., "Semiclassical gravity and mesoscopic physics", in Feng, D.S., and Hu, B.L., eds., Quantum Classical Correspondence, Proceedings of the 4th Drexel Symposium on Quantum Nonintegrability, Drexel University, Philadelphia, USA, September 8-11, 1994, (International Press, Cambridge, U.S.A., 1997). 6b, 8 
[187] Hu, B.L., "Stochastic gravity", Int. J. Theor. Phys., 38, 2987-3037, (1999). Related online version (cited on 31 March 2003): http://arXiv.org/abs/gr-qc/9902064. 1, 6(c)i, 3.2, 4, 5, 8.1.1, 8.4, 9

[188] Hu, B.L., "A kinetic theory approach to quantum gravity", Int. J. Theor. Phys., 41, 20912119, (2002). Related online version (cited on 31 March 2003): http://arXiv.org/abs/gr-qc/0204069. 6(c)i, 3.2, 5, 9

[189] Hu, B.L., "Can spacetime be a condensate?", Int. J. Theor. Phys., 44, 1785-1806, (2005). Related online version (cited on 22 January 2008):

http://arXiv.org/abs/gr-qc/0503067. 6a

[190] Hu, B.L., "New View on Quantum Gravity and the Origin of the Universe", in Where Do We Come From? On the Origin of the Universe, (Commercial Press, Hong Kong, 2007). Related online version (cited on 22 January 2008): http://arXiv.org/abs/gr-qc/0611058. In Chinese. 6b, 8, 9

[191] Hu, B.L., and Matacz, A., "Quantum Brownian motion in a bath of parametric oscillators: A Model for system-field interactions", Phys. Rev. D, 49, 6612-6635, (1994). Related online version (cited on 31 March 2003):

http://arXiv.org/abs/gr-qc/9312035. 4

[192] Hu, B.L., and Matacz, A., "Back reaction in semiclassical cosmology: The Einstein-Langevin equation", Phys. Rev. D, 51, 1577-1586, (1995). Related online version (cited on 31 March 2003):

http://arXiv.org/abs/gr-qc/9403043. 2c, 2.1, 8.3.2

[193] Hu, B.L., and Parker, L., "Effect of graviton creation in isotropically expanding universes", Phys. Lett. A, 63, 217-220, (1977). 1(b)ii, 2, 4

[194] Hu, B.L., and Parker, L., "Anisotropy damping through quantum effects in the early universe", Phys. Rev. D, 17, 933-945, (1978). 1(b)ii, 2, 4

[195] Hu, B.L., Paz, J.P., and Sinha, S., "Minisuperspace as a Quantum Open System", in Hu, B.L., Ryan, M.P., and Vishveswara, C.V., eds., Directions in General Relativity, Vol. 1, Proceedings of the 1993 International Symposium, Maryland: Papers in honor of Charles Misner, pp. 145-165, (Cambridge University Press, Cambridge, U.K.; New York, U.S.A., 1993). 1d, 4

[196] Hu, B.L., Paz, J.P., and Zhang, Y., "Quantum Brownian motion in a general environment: 1. Exact master equation with nonlocal dissipation and colored noise", Phys. Rev. D, 45, 2843-2861, (1992). 8.2.3, 8.2.4, 8.2.6

[197] Hu, B.L., Paz, J.P., and Zhang, Y., "Quantum Brownian motion in a general environment. 2: Nonlinear coupling and perturbative approach", Phys. Rev. D, 47, 1576-1594, (1993). 8.2.3, 8.2.4, 8.2.6

[198] Hu, B.L., and Phillips, N.G., "Fluctuations of energy density and validity of semiclassical gravity", Int. J. Theor. Phys., 39, 1817-1830, (2000). Related online version (cited on 31 March 2003):

http://arXiv.org/abs/gr-qc/0004006. 3a, 2.1, 3.1, 3.3, 5

[199] Hu, B.L., Raval, A., and Sinha, S., "Notes on black hole fluctuations and backreaction", in Bhawal, B., and Iyer, B.R., eds., Black Holes, Gravitational Radiation and the Universe: Essays in Honour of C. V. Vishveshwara, Fundamental Theories of Physics, (Kluwer, Dordrecht, Netherlands; Boston, U.S.A., 1999). 4e, 8.1.1, 8.1.2, 8.3, 8.3.2 
[200] Hu, B.L., and Roura, A., "Black Hole Fluctuations and Dynamics from Back-Reaction of Hawking Radiation: Current Work and Further Studies Based on Stochastic Gravity", in Nester, J.M., Chen, C.-M., and Hsu, J.P., eds., Gravitation and Astrophysics: On the Occasion of the 90th Year of General Relativity, Proceedings of the VII Asia-Pacific International Conference, National Central University, Taiwan, 23-26 November 2005, pp. 236-250, (World Scientific, Singapore; Hackensack, U.S.A., 2007). 4e, 8.3

[201] Hu, B.L., and Roura, A., "Fluctuations of an evaporating black hole from back reaction of its Hawking radiation: Questioning a premise in earlier work", Int. J. Theor. Phys., 46, 2204-2217, (2007). Related online version (cited on 22 January 2008): http://arXiv.org/abs/gr-qc/0601088. 4e, 8.1.1

[202] Hu, B.L., and Roura, A., "Metric fluctuations of an evaporating black hole from back reaction of stress tensor fluctuations", Phys. Rev. D, 76, 124018, (2007). Related online version (cited on 22 January 2008):

http://arXiv.org/abs/arXiv:0708.3046. 8.1.1, 8.3, 8.3.2, 8.3.2, 8.3.3

[203] Hu, B.L., Roura, A., and Verdaguer, E., "Induced quantum metric fluctuations and the validity of semiclassical gravity", Phys. Rev. D, 70, 044002, 1-24, (2004). Related online version (cited on 31 March 2003):

http://arXiv.org/abs/gr-qc/0402029. 3a, 2.1, 3.1, 3.2, 3.3, 3.3.1, 6.5.1, 6.5.2, 6.5.4, 7.4

[204] Hu, B.L., Roura, A., and Verdaguer, E., "Stability of semiclassical gravity solutions with respect to quantum metric fluctuations", Int. J. Theor. Phys., 43, 749-766, (2004). Related online version (cited on 22 January 2008):

http://arXiv.org/abs/gr-qc/0508010. 3a, 2.1, 3.1, 3.3, 3.3.1, 7.4

[205] Hu, B.L., and Shiokawa, K., "Wave propagation in stochastic spacetimes: Localization, amplification and particle creation", Phys. Rev. D, 57, 3474-3483, (1998). Related online version (cited on 31 March 2003):

http://arXiv.org/abs/gr-qc/9708023.4a, 8.4

[206] Hu, B.L., and Sinha, S., "A fluctuation-dissipation relation for semiclassical cosmology", Phys. Rev. D, 51, 1587-1606, (1995). Related online version (cited on 31 March 2003): http://arXiv.org/abs/gr-qc/9403054. 2a, 2c, 2.1, 4, 4.1, 4.2, 5.2.3, 8.1.2, 8.3.2

[207] Hu, B.L., and Verdaguer, E., "Recent Advances in Stochastic Gravity: Theory and Issues", in Bergmann, P.G., and De Sabbata, V., eds., Advances in the interplay between quantum and gravity physics, Proceedings of the NATO Advanced Study Institute, held in Erice, Italy, April 30 - May 10, 2001, NATO Science Series II, vol. 60, pp. 133-218, (Kluwer, Dordrecht, Netherlands; Boston, U.S.A., 2002). Related online version (cited on 31 March 2003): http://arXiv.org/abs/gr-qc/0110092. 1, 2.1, 3.1, 8.1.1

[208] Hu, B.L., and Verdaguer, E., "Stochastic gravity: A primer with applications", Class. Quantum Grav., 20, R1-R42, (2003). Related online version (cited on 31 March 2003): http://arXiv.org/abs/gr-qc/0211090. 1, 3.2, 4.3.1, 6.3, 8.1, 8.1.1

[209] Isham, C.J., "Quantum logic and the histories approach to quantum theory", J. Math. Phys., 35, 2157-2185, (1994). Related online version (cited on 31 March 2003): http://arXiv.org/abs/gr-qc/9308006. 1(c)iii, 2.1

[210] Isham, C.J., and Linden, N., "Quantum temporal logic and decoherence functionals in the histories approach to generalized quantum theory", J. Math. Phys., 35, 5452-5476, (1994). Related online version (cited on 31 March 2003):

http://arXiv.org/abs/gr-qc/9405029. 1(c)iii, 2.1 
[211] Isham, C.J., and Linden, N., "Continuous histories and the history group in generalized quantum theory", J. Math. Phys., 36, 5392-5408, (1995). Related online version (cited on 31 March 2003):

http://arXiv.org/abs/gr-qc/9503063. 1(c)iii, 2.1

[212] Isham, C.J., Linden, N., Savvidou, K., and Schreckenberg, S., "Continuous time and consistent histories", J. Math. Phys., 39, 1818-1834, (1998). Related online version (cited on 31 March 2003):

http://arXiv.org/abs/quant-ph/9711031. 1(c)iii, 2.1

[213] Israel, W., "Thermo field dynamics of black holes", Phys. Lett. A, 57, 107-110, (1976). 2, 8

[214] Jacobson, T., "On the nature of black hole entropy", in Burgess, C.P., and Myers, R.C., eds., General Relativity and Relativistic Astrophysics, Eight Canadian Conference, Montréal, Québec June 1999, AIP Conference Proceedings, vol. 493, (American Institute of Physics, Melville, U.S.A., 1999). 8

[215] Jacobson, T.A., "Black hole radiation in the presence of a short distance cutoff", Phys. Rev. $D, 48,728-741$, (1993). Related online version (cited on 22 January 2008):

http://arXiv.org/abs/hep-th/9303103. 8

[216] Jacobson, T.A., "Thermodynamics of space-time: The Einstein equation of state", Phys. Rev. Lett., 75, 1260-1263, (1995). Related online version (cited on 22 January 2008): http://arXiv.org/abs/gr-qc/9504004.6a, 8

[217] Jensen, B.P., McLaughlin, J.G., and Ottewill, A.C., "One loop quantum gravity in Schwarzschild space-time", Phys. Rev. D, 51, 5676-5697, (1995). Related online version (cited on 31 March 2003):

http://arXiv.org/abs/gr-qc/9412075. 8.1.1

[218] Jensen, B.P., and Ottewill, A.C., "Renormalized electromagnetic stress tensor in Schwarzschild space-time", Phys. Rev. D, 39, 1130-1138, (1989). 8.1 .1

[219] Johnson, P.R., and Hu, B.L., "Stochastic theory of relativistic particles moving in a quantum field: Scalar Abraham-Lorentz-Dirac-Langevin equation, radiation reaction, and vacuum fluctuations", Phys. Rev. D, 65, 065015, 1-24, (2002). Related online version (cited on 31 March 2003):

http: //arXiv.org/abs/quant-ph/0101001. 3.2

[220] Jones, D.S., Generalised Functions, European Mathematics Series, (McGraw-Hill, London, U.K.; New York, U.S.A., 1966). 6.3

[221] Joos, E., and Zeh, H.D., "The Emergence of classical properties through interaction with the environment", Z. Phys. B, 59, 223-243, (1985). 1(c)iii, 2.1, 4

[222] Jordan, R.D., "Effective field equations for expectation values", Phys. Rev. D, 33, 444-454, (1986). 1, 1(b)iv, 2a, 4

[223] Jordan, R.D., "Stability of flat space-time in quantum gravity", Phys. Rev. D, 36, 3593-3603, (1987). 1, 1(b)iii, 2a, 3a, 3.3, 4, 6.3, 6.4.1

[224] Kabat, D., Shenker, S.H., and Strassler, M.J., "Black hole entropy in the $O(N)$ model", Phys. Rev. D, 52, 7027-7036, (1995). Related online version (cited on 31 March 2003):

http://arXiv.org/abs/hep-th/9506182. 8 
[225] Kahya, E.O., and Woodard, R.P., "Quantum Gravity Corrections to the One Loop Scalar Self-Mass during Inflation", Phys. Rev., 76, 124005, (2007). Related online version (cited on 22 January 2008):

http://arXiv.org/abs/arXiv:0709.0536. 7.4

[226] Kahya, E.O., and Woodard, R.P., "Scalar Field Equations from Quantum Gravity during Inflation", (2007). URL (cited on 22 January 2008):

http://arXiv.org/abs/arXiv:0710.5282. 7.4

[227] Keldysh, L.V., "Diagram technique for nonequilibrium processes", Zh. Eksp. Teor. Fiz., 47, 1515-1527, (1964). 1, 1(b)iii, 4

[228] Kent, A., "Quasiclassical Dynamics in a Closed Quantum System", Phys. Rev. A, 54, 46704675, (1996). Related online version (cited on 31 March 2003):

http://arXiv.org/abs/gr-qc/9512023. 1(c)iii, 2.1

[229] Kent, A., "Consistent sets contradict", Phys. Rev. Lett., 78, 2874-2877, (1997). Related online version (cited on 31 March 2003):

http://arXiv.org/abs/gr-qc/9604012. 1(c)iii, 2.1

[230] Kent, A., "Consistent Sets and Contrary Inferences in Quantum Theory: Reply to Griffiths and Hartle", Phys. Rev. Lett., 81, 1982, (1998). Related online version (cited on 31 March 2003):

http://arXiv.org/abs/gr-qc/9808016. 1(c)iii, 2.1

[231] Kiefer, C., "Continuous measurement of mini-superspace variables by higher multipoles", Class. Quantum Grav., 4, 1369-1382, (1987). 1d, 4

[232] Kirsten, K., Spectral Functions in Mathematics and Physics, (Chapman and Hall/CRC, Boca Raton, U.S.A., 2001). 5

[233] Kolb, E.W., and Turner, M.S., The Early Universe, Frontiers in Physics, vol. 69, (AddisonWesley, Reading, U.S.A., 1990). 7, 7.3

[234] Kubo, R., "Statistical-mechanical theory of irreversible processes. I. General theory and simple applications to magnetic and conduction problems", J. Phys. Soc. Jpn., 12, 570-586, (1957). 8.1.2

[235] Kubo, R., "The fluctuation-dissipation theorem", Rep. Prog. Phys., 29, 255-284, (1966). 8.1.2

[236] Kubo, R., Toda, M., and Hashitsume, N., Statistical Physics, (Springer, Berlin, Germany, 1985). 8.1.2

[237] Kuo, C., and Ford, L.H., "Semiclassical gravity theory and quantum fluctuations", Phys. Rev. D, 47, 4510-4519, (1993). Related online version (cited on 31 March 2003): http://arXiv.org/abs/gr-qc/9304008. 3a, 2.1, 3.1, 3.3, 5

[238] Landau, L.D., Lifshitz, E.M., and Pitaevskii, L.P., Statistical Physics, Part 2, Course of Theoretical Physics, vol. 9, (Pergamon Press, Oxford, U.K.; New York, U.S.A., 1980). 8.1.2

[239] Lee, D.-S., and Boyanovsky, D., "Dynamics of phase transitions induced by a heat bath", Nucl. Phys. B, 406, 631-654, (1993). Related online version (cited on 31 March 2003): http://arXiv.org/abs/hep-ph/9304272. 4 
[240] Levin, M.A., and Wen, X.-G., "Photons and electrons as emergent phenomena", Rev. Mod. Phys., 77, 871-879, (2005). Related online version (cited on 22 January 2008):

http://arXiv.org/abs/cond-mat/0407140. 6a, 8

[241] Lifshitz, E., "On the gravitational stability of the expanding universe", J. Phys. (Moscow), 10, 116, (1946). 7

[242] Lifshitz, E. M., and Khalatnikov, I. M., "Investigations in relativistic cosmology", Adv. Phys., 12, 185-249, (1963). 7

[243] Linde, A.D., "Coleman-Weinberg theory and a new inflationary universe scenario", Phys. Lett. B, 114, 431-435, (1982). 2, 7

[244] Linde, A.D., "Initial conditions for inflation", Phys. Lett. B, 162, 281-286, (1985). 2, 7

[245] Linde, A.D., Particle Physics and Inflationary Cosmology, Contemporary Concepts in Physics, vol. 5, (Harwood, Chur, Switzerland; New York, U.S.A., 1990). 7, 7.1

[246] Lindenberg, K., and West, B.J., The Nonequilibrium Statistical Mechanics of Open and Closed Systems, (VCH Publishers, New York, U.S.A., 1990). 1, 1(c)i

[247] Lombardo, F.C., and Mazzitelli, F.D., "Coarse graining and decoherence in quantum field theory", Phys. Rev. D, 53, 2001-2011, (1996). Related online version (cited on 31 March 2003): http://arXiv.org/abs/hep-th/9508052. 4

[248] Lombardo, F.C., and Mazzitelli, F.D., "Einstein-Langevin equations from running coupling constants", Phys. Rev. D, 55, 3889-3892, (1997). Related online version (cited on 31 March 2003):

http://arXiv.org/abs/gr-qc/9609073. 2c, 2.1, 8.3.2

[249] Lombardo, F.C., Mazzitelli, F.D., and Russo, J.G., "Energy-momentum tensor for scalar fields coupled to the dilaton in two dimensions", Phys. Rev. D, 59, 064007, (1999). Related online version (cited on 22 January 2008):

http://arXiv.org/abs/gr-qc/9808048. 8.3.2

[250] Lowe, D.A., and Thorlacius, L., "Comments on the black hole information problem", Phys. Rev. D, 73, 104027, (2006). Related online version (cited on 22 January 2008): http://arXiv.org/abs/hep-th/0601059. 8

[251] Lukash, V.N., and Starobinsky, A.A., "Isotropization of cosmological expansion due to particle creation effect", Sov. Phys. JETP, 39, 742, (1974). 1(b)ii, 2, 4

[252] Maia, C., and Schützhold, R., "Quantum toy model for black-hole back-reaction", Phys. Rev. $D, \mathbf{7 6}, 101502,(2007)$. Related online version (cited on 22 January 2008): http://arXiv.org/abs/arXiv:0706.4010. 1(b)ii

[253] Maldacena, J.M., "Black holes and D-branes", Nucl. Phys. A (Proc. Suppl.), 61, 111-123, (1998). Related online version (cited on 31 March 2003): http://arXiv.org/abs/hep-th/9705078. 8

[254] Maldacena, J.M., Strominger, A., and Witten, E., "Black hole entropy in M-Theory", J. High Energy Phys., 1997(12), 002, (1997). Related online version (cited on 31 March 2003): http://arXiv.org/abs/hep-th/9711053. 8 
[255] Marolf, D., "On the Quantum Width of a Black Hole Horizon", in Trampetić, J., and Wess, J., eds., Particle Physics and the Universe, Proceedings of the 9th Adriatic Meeting, September 2003, Dubrovnik, Springer Proceedings in Physics, vol. 98, pp. 99-112, (Springer, Berlin, Germany; New York, U.S.A., 2005). Related online version (cited on 22 January 2008): http://arXiv.org/abs/hep-th/0312059. 8.3, 8.3.2

[256] Martín, R., and Verdaguer, E., "An effective stochastic semiclassical theory for the gravitational field", Int. J. Theor. Phys., 38, 3049-3089, (1999). Related online version (cited on 31 March 2003):

http://arXiv.org/abs/gr-qc/9812063. 2c, 2.1, 4

[257] Martín, R., and Verdaguer, E., "On the semiclassical Einstein-Langevin equation", Phys. Lett. B, 465, 113-118, (1999). Related online version (cited on 31 March 2003):

http://arXiv.org/abs/gr-qc/9811070. 1, 2c, 2.1, 3, 8.1.3, 8.2.6

[258] Martín, R., and Verdaguer, E., "Stochastic semiclassical gravity", Phys. Rev. D, 60, 084008, 1-24, (1999). Related online version (cited on 31 March 2003):

http://arXiv.org/abs/gr-qc/9904021. 1, 2c, 2.1, 3.1, 3.2, 4, 5, 5.2.3

[259] Martín, R., and Verdaguer, E., "Stochastic semiclassical fluctuations in Minkowski spacetime", Phys. Rev. D, 61, 124024, 1-26, (2000). Related online version (cited on 31 March 2003):

http://arXiv.org/abs/gr-qc/0001098. 2d, 2.1, 3.2, 3.3.1, 6, 6.1, 6.2, 6.2, 6.3, 6.3, 6.4.1, 6.4.3, 6.4.3, 6.5.1, 6.5.2, 8.1.3

[260] Massar, S., "The semiclassical back reaction to black hole evaporation", Phys. Rev. D, 52, 5857-5864, (1995). Related online version (cited on 31 March 2003):

http://arXiv.org/abs/gr-qc/9411039. 8.1.1, 8.3, 8.3.1, 8.3.3

[261] Massar, S., and Parentani, R., "How the change in horizon area drives black hole evaporation", Nucl. Phys. B, 575, 333-356, (2000). Related online version (cited on 31 March 2003): http://arXiv.org/abs/gr-qc/9903027.4b, 5a

[262] Matacz, A., "Inflation and the fine-tuning problem", Phys. Rev. D, 56, 1836-1840, (1997). Related online version (cited on 31 March 2003):

http://arXiv.org/abs/gr-qc/9611063. 4d, 7.3

[263] Matacz, A., "A New Theory of Stochastic Inflation", Phys. Rev. D, 55, 1860-1874, (1997). Related online version (cited on 31 March 2003): http://arXiv.org/abs/gr-qc/9604022. 4d, 7.3

[264] Mazur, P.O., and Mottola, E., "Gravitational vacuum condensate stars", Proc. Natl. Acad. Sci. USA, 101, 9545-9550, (2004). Related online version (cited on 22 January 2008): http://arXiv.org/abs/gr-qc/0407075. 8.3, 8.3.2

[265] Misner, C.W., "Mixmaster Universe", Phys. Rev. Lett., 22, 1071-1074, (1969). 2

[266] Misner, C.W., Thorne, K.S., and Wheeler, J.A., Gravitation, (W.H. Freeman, San Francisco, U.S.A., 1973). 1a, 1

[267] Morikawa, M., "Classical fluctuations in dissipative quantum systems", Phys. Rev. D, 33, $3607-3612,(1986) .4$

[268] Mottola, E., "Quantum fluctuation-dissipation theorem for general relativity", Phys. Rev. $D, \mathbf{3 3}, 2136-2146,(1986)$. 2a, 4e, 8.1.2, 8.2.6 
[269] Mukhanov, V., Physical Foundations of Cosmology, (Cambridge University Press, Cambridge, U.K., 2005). 7

[270] Mukhanov, V.F., Feldman, H.A., and Brandenberger, R.H., "Theory of cosmological perturbations. Part 1. Classical perturbations. Part 2. Quantum theory of perturbations. Part 3. Extensions", Phys. Rep., 215, 203-333, (1992). 7, 7.2, 7.2, 7.3

[271] Ng, Y.J., "Selected topics in Planck-scale physics", Mod. Phys. Lett. A, 18, 1073-1098, (2003). Related online version (cited on 22 January 2008):

http://arXiv.org/abs/gr-qc/0305019. 5b

[272] Nicolai, H., and Peeters, K., "Loop and Spin Foam Quantum Gravity: A Brief Guide for Beginners", in Stamatescu, I.-O., and Seiler, E., eds., Approaches to Fundamental Physics: An Assessment of Current Theoretical Ideas, Lecture Notes in Physics, vol. 721, pp. 151-184, (Springer, Berlin, Germany; New York, U.S.A., 2007). Related online version (cited on 22 January 2008):

http://arXiv.org/abs/hep-th/0601129. 5b

[273] Niemeyer, J.C., and Parentani, R., "Trans-Planckian dispersion and scale invariance of inflationary perturbations", Phys. Rev. D, 64, 101301, 1-4, (2001). Related online version (cited on 31 March 2003):

http://arXiv.org/abs/astro-ph/0101451. 5a

[274] Nyquist, H., "Thermal agitation of electric charge in conductors", Phys. Rev., 32, 110-113, (1928). 8.1.2

[275] Omnès, R., "Logical reformulation of quantum mechanics. 1. Foundations", J. Stat. Phys., 53, 893-932, (1988). 1(c)iii, 2.1

[276] Omnès, R., "Logical reformulation of quantum mechanics. 2. Interferences and the EinsteinPodolsky-Rosen experiment", J. Stat. Phys., 53, 933-955, (1988). 1(c)iii, 2.1

[277] Omnès, R., "Logical reformulation of quantum mechanics. 3. Classical limit and irreversibility", J. Stat. Phys., 53, 957-975, (1988). 1(c)iii, 2.1

[278] Omnès, R., "From Hilbert space to common sense: A synthesis of recent progress in the interpretation of quantum mechanics", Ann. Phys. (N.Y.), 201, 354-447, (1990). 1(c)iii, 2.1

[279] Omnès, R., "Consistent interpretations of quantum mechanics", Rev. Mod. Phys., 64, 339382, (1992). 1(c)iii, 2.1

[280] Omnès, R., The Interpretation of Quantum Mechanics, (Princeton University Press, Princeton, U.S.A., 1994). 1(c)iii, 2.1

[281] Oriti, D., Approaches to Quantum Gravity: Toward a New Understanding of Space, Time, and Matter, (Cambridge University Press, Cambridge, England, U.K., 2008). 6(c)iii

[282] Osborn, H., and Shore, G.M., "Correlation functions of the energy momentum tensor on spaces of constant curvature", Nucl. Phys. B, 571, 287-357, (2000). Related online version (cited on 31 March 2003):

http://arXiv.org/abs/hep-th/9909043. 2.1

[283] Padmanabhan, T., "Decoherence in the density matrix describing quantum three geometries and the emergence of classical space-time", Phys. Rev. D, 39, 2924-2932, (1989). 1d, 4 
[284] Padmanabhan, T., Structure Formation in the Universe, (Cambridge University Press, Cambridge, U.K.; New York, U.S.A., 1993). 7, 7.3

[285] Page, D.N., "Particle Emission Rates from a Black Hole: Massless Particles from an Uncharged, Nonrotating Hole", Phys. Rev. D, 13, 198-206, (1976). 8.3.1

[286] Page, D.N., "Is black hole evaporation predictable?", Phys. Rev. Lett., 44, 301-304, (1980). 8

[287] Page, D.N., "Thermal stress tensors in static Einstein spaces", Phys. Rev. D, 25, 1499-1509, (1982). 8.1.1, 8.2, 8.2.1, 8.2.4, 8.2.6, 8.3.1

[288] Page, D.N., "Black hole information", in Mann, R.B., and McLenhagan, R.G., eds., General Relativity and Relativistic Astrophysics, Proceedings of the 5th Canadian Conference on General Relativity and Relativistic Astrophysics, University of Waterloo, 13-15 May, 1993, (World Scientific, Singapore; River Edge, U.S.A., 1994). Related online version (cited on 9 May 2005):

http://arxiv.org/abs/hep-th/9305040. 8

[289] Parentani, R., "Quantum metric fluctuations and Hawking radiation", Phys. Rev. D, 63, 041503, 1-4, (2001). Related online version (cited on 31 March 2003):

http://arXiv.org/abs/gr-qc/0009011. 4b, 5a, 8.3.2

[290] Parentani, R., "Toward a collective treatment of quantum gravitational interactions", Int. J. Theor. Phys., 40, 2201-2216, (2001). 8.3.2, 8.3.2, 8.3.2

[291] Parentani, R., "Beyond the semi-classical description of black hole evaporation", Int. J. Theor. Phys., 41, 2175-2200, (2002). Related online version (cited on 22 January 2008): http://arXiv.org/abs/arXiv:0704.2563. 8.3.2, 8.3.2, 8.3.2

[292] Parentani, R., and Piran, T., "The internal geometry of an evaporating black hole", Phys. Rev. Lett., 73, 2805-2808, (1994). Related online version (cited on 31 March 2003): http://arXiv.org/abs/hep-th/9405007. 8.1.1

[293] Parker, L., "Quantized Fields and Particle Creation in Expanding Universes. I", Phys. Rev., 183, 1057-1068, (1969). 2

[294] Parker, L., "Probability distribution of particles created by a black hole", Phys. Rev. D, 12, 1519-1525, (1975). 2, 8

[295] Parker, L., and Simon, J.Z., "Einstein equation with quantum corrections reduced to second order", Phys. Rev. D, 47, 1339-1355, (1993). Related online version (cited on 22 January 2008):

http://arXiv.org/abs/gr-qc/9211002. 3.3, 6.5.1

[296] Paz, J.P., "Anisotropy dissipation in the early Universe: Finite-temperature effects reexamined", Phys. Rev. D, 41, 1054-1066, (1990). 1, 1(b)iii, 2a, 4

[297] Paz, J.P., "Decoherence and back reaction: The origin of the semiclassical Einstein equations", Phys. Rev. D, 44, 1038-1049, (1991). 2.1, 4

[298] Paz, J.P., and Sinha, S., "Decoherence and back reaction in quantum cosmology: Multidimensional minisuperspace examples", Phys. Rev. D, 45, 2823-2842, (1992). 2.1, 4 
[299] Paz, J.P., and Zurek, W.H., "Environment induced decoherence, classicality and consistency of quantum histories", Phys. Rev. D, 48, 2728-2738, (1993). Related online version (cited on 31 March 2003):

http://arXiv.org/abs/gr-qc/9304031. 1(c)iii, 2.1

[300] Peebles, P.J.E., Large Scale Structure of the Universe, (Princeton University Press, Princeton, U.S.A., 1980). 7

[301] Phillips, N.G., "Symbolic computation of higher order correlation functions of quantum fields in curved spacetimes", unknown status. in preparation. 5.1.1

[302] Phillips, N.G., and Hu, B.L., "Fluctuations of the vacuum energy density of quantum fields in curved spacetime via generalized zeta functions", Phys. Rev. D, 55, 6123-6134, (1997). Related online version (cited on 31 March 2003):

http://arXiv .org/abs/gr-qc/9611012. 2.1, 3.1, 5, 5.2.2

[303] Phillips, N.G., and Hu, B.L., "Vacuum energy density fluctuations in Minkowski and Casimir states via smeared quantum fields and point separation", Phys. Rev. D, 62, 084017, 1-18, (2000). Related online version (cited on 31 March 2003):

http://arXiv.org/abs/gr-qc/0005133. 3a, 2.1, 3.1, 3.1, 3.3, 5

[304] Phillips, N.G., and Hu, B.L., "Noise kernel in stochastic gravity and stress energy bitensor of quantum fields in curved spacetimes", Phys. Rev. D, 63, 104001, 1-16, (2001). Related online version (cited on 31 March 2003):

http://arXiv.org/abs/gr-qc/0010019. 1, 3c, 2.1, 3.1, 3.1, 3.2, 3.2, 3.2, 5, 8.1.3, 8.2.4

[305] Phillips, N.G., and Hu, B.L., "Noise kernel and the stress energy bitensor of quantum fields in hot flat space and the Schwarzschild black hole under the Gaussian approximation", Phys. Rev. D, 67, 104002, 1-26, (2003). Related online version (cited on 31 March 2003): http://arXiv.org/abs/gr-qc/0209056. 1, 3c, 4b, 2.1, 3.1, 3.2, 3.2, 5, 5.2.3, 8.1.3, 8.2, 8.2.4, 8.2.6, 8.3.2

[306] Polchinski, J., and Strominger, A., "A Possible resolution of the black hole information puzzle", Phys. Rev. D, 50, 7403-7409, (1994). Related online version (cited on 22 January 2008):

http://arXiv.org/abs/hep-th/9407008. 8

[307] Preskil, J., "Do black holes destroy information?", in Kalara, S., and Nanopoulos, D.V., eds., Black Holes, Membranes, Wormholes and Superstrings, Proceedings of the International Symposium, Houston Advanced Research Center, USA, 16 - 18 January 1992, (World Scientific, Singapore; River Edge, U.S.A., 1993). Related online version (cited on 22 January 2008):

http://arXiv.org/abs/hep-th/9209058. 8

[308] Ramsey, S.A., Hu, B.L., and Stylianopoulos, A.M., "Nonequilibrium inflaton dynamics and reheating. II: Fermion production, noise, and stochasticity", Phys. Rev. D, 57, 6003-6021, (1998). Related online version (cited on 31 March 2003):

http://arXiv.org/abs/hep-ph/9709267. 4.2

[309] Randjbar-Daemi, S., "Stability of the Minskowski vacuum in the renormalized semiclassical theory of gravity", J. Phys. A, 14, L229-L233, (1981). 6.4.1

[310] Randjbar-Daemi, S., "A recursive formula for the evaluation of the diagonal matrix elements of the stress energy tensor operator and its application in the semiclassical theory of gravity", J. Phys. A, 15, 2209-2219, (1982). 6.4.1 
[311] Rebhan, A., "Collective phenomena and instabilities of perturbative quantum gravity at nonzero temperature", Nucl. Phys. B, 351, 706-734, (1991). 8.2, 8.2.3, 8.2.3, 8.2.3, 8.2.3

[312] Rebhan, A., "Analytical solutions for cosmological perturbations with relativistic collisionless matter", Nucl. Phys. B, 368, 479-508, (1992). 8.2, 8.2.3, 8.2.3, 8.2.3, 8.2.3

[313] Roura, A., and Verdaguer, E., "Mode decomposition and renormalization in semiclassical gravity", Phys. Rev. D, 60, 107503, 1-4, (1999). Related online version (cited on 31 March 2003):

http://arXiv.org/abs/gr-qc/9906036. 2.1

[314] Roura, A., and Verdaguer, E., "Spacelike fluctuations of the stress tensor for de Sitter vacuum", Int. J. Theor. Phys., 38, 3123-3133, (1999). Related online version (cited on 31 March 2003):

http://arXiv.org/abs/gr-qc/9904039. 7.2, 7.4

[315] Roura, A., and Verdaguer, E., "Semiclassical cosmological perturbations generated during inflation", Int. J. Theor. Phys., 39, 1831-1839, (2000). 3.2, 7, 7.3

[316] Roura, A., and Verdaguer, E., "Cosmological perturbations from stochastic gravity", (2007). URL (cited on 22 January 2008):

http://arXiv.org/abs/arXiv:0709.1940. 4d, 2.1, 7, 7.2, 7.2, 7.3, 7.3, 7.4, 8.1.3

[317] Roura, A., and Verdaguer, E., "Stochastic gravity as the large $N$ limit for quantum metric fluctuations", unknown status, (2008). in preparation. 2.1, 6.4.3, 7.4

[318] Russo, J.G., Susskind, L., and Thorlacius, L., "Black hole evaporation in $1+1$ dimensions", Phys. Lett. B, 292, 13-18, (1992). Related online version (cited on 22 January 2008):

http://arXiv.org/abs/hep-th/9201074. 8

[319] Russo, J.G., Susskind, L., and Thorlacius, L., "The Endpoint of Hawking radiation", Phys. Rev. D, 46, 3444-3449, (1992). Related online version (cited on 22 January 2008): http://arXiv.org/abs/hep-th/9206070. 8

[320] Schützhold, R., "Effective horizons in the laboratory", in Unruh, W.G., and Schützhold, R., eds., Quantum Analogues: From Phase Transitions to Black Holes and Cosmology, Selected lectures from the international workshop on "Quantum Simulations via Analogues", held at the Max Planck Institute for the Physics of Complex Systems in Dresden, Germany, July 25-28, 2005, Lecture Notes in Physics, vol. 718, pp. 5-30, (Springer, Berlin, Germany; New York, U.S.A., 2007). 1(b)ii, 8

[321] Schützhold, R., Uhlmann, M., Petersen, L., Schmitz, H., Friedenauer, A., and Schutz, T., "Analogue of cosmological particle creation in an ion trap", Phys. Rev. Lett., 99, 201301, (2007). Related online version (cited on 22 January 2008):

http://arXiv.org/abs/arXiv:0705.3755. 1(b)ii

[322] Schwartz, L., Théorie des distributions, (Hermann, Paris, France, 1978). 6.4.2

[323] Schwinger, J.S., "Brownian motion of a quantum oscillator", J. Math. Phys., 2, 407-432, (1961). 1, 1(b)iii, 4

[324] Sciama, D.W., "Thermal and quantum fluctuations in special and general relativity: an Einstein Synthesis", in de Finis, F., ed., Centenario di Einstein: Relativity, quanta, and cosmology in the development of the scientific thought of Albert Einstein, (Editrici Giunti Barbera Universitaria, Florence, Italy, 1979). 4e, 8.1.2, 8.2.6 
[325] Sciama, D.W., Candelas, P., and Deutsch, D., "Quantum field theory, horizons and thermodynamics", Adv. Phys., 30, 327-366, (1981). 4e, 8.1.2, 8.2.6

[326] Seiberg, N., "Emergent spacetime", in Gross, D., Henneaux, M., and Sevrin, A., eds., The Quantum Structure Of Space And Time, Proceedings of the 23rd Solvay Conference on Physics, Brussels, Belgium, 1-3 December, 2005, (World Scientific, Singapore; Hackensack, U.S.A., 2007). Related online version (cited on 22 January 2008):

http://arXiv.org/abs/hep-th/0601234. 6a, 8

[327] Sexl, R.U., and Urbantke, H.K., "Production of particles by gravitational fields", Phys. Rev., 179, 1247-1250, (1969). 2

[328] Shiokawa, K., "Mesoscopic fluctuations in stochastic spacetime", Phys. Rev. D, 62, 024002, 1-14, (2000). Related online version (cited on 31 March 2003):

http://arXiv.org/abs/hep-th/0001088. 5c, 8.4

[329] Simon, J.Z., "Higher derivative Lagrangians, nonlocality, problems and solutions", Phys. Rev. D, 41, 3720-3733, (1990). 3a, 3.3

[330] Simon, J.Z., "Stability of flat space, semiclassical gravity, and higher derivatives", Phys. Rev. D, 43, 3308-3316, (1991). 3.3, 6.4.1

[331] Sinha, S., and Hu, B.L., "Validity of the minisuperspace approximation: An Example from interacting quantum field theory", Phys. Rev. D, 44, 1028-1037, (1991). 4

[332] Sinha, S., Raval, A., and Hu, B.L., "Black Hole Fluctuations and Backreaction in Stochastic Gravity", Found. Phys., 33, 37-64, (2003). 4e, 4, 8.1.1, 8.2

[333] Smolin, J.A., and Oppenheim, J., "Information locking in black holes", Phys. Rev. Lett., 96, 081302, (2006). Related online version (cited on 22 January 2008):

http://arXiv.org/abs/hep-th/0507287. 8

[334] Smoot, G.F., Bennett, C.L., Kogut, A., Wright, E.L., Aymon, J., Boggess, N.W., Cheng, E.S., de Amici, G., Gulkis, S., Hauser, M.G., Hinshaw, G., Jackson, P.D., Janssen, M., Kaita, E., Kelsall, T., Keegstra, P., Lineweaver, C.H., Loewenstein, K., Lubin, P., Mather, J., Meyer, S.S., Moseley, S.H., Murdock, T., Rokke, L., Silverberg, R.F., Tenorio, L., Weiss, R., and Wilkinson, D.T., "Structure in the COBE differential microwave radiometer first-year maps", Astrophys. J. Lett., 396, L1-L5, (1992). 7.1, 7.3

[335] Sorkin, R.D., "Two Topics concerning Black Holes: Extremality of the Energy, Fractality of the Horizon", in Fulling, S.A., ed., Heat Kernel Techniques and Quantum Gravity, Winnipeg, Canada, August, 1994, Discourses Math. Appl., vol. 4, pp. 387-407, (University of Texas Press, College Station, U.S.A., 1995). Related online version (cited on 22 January 2008): http://arXiv.org/abs/gr-qc/9508002. 8.3

[336] Sorkin, R.D., "How Wrinkled is the Surface of a Black Hole?", in Wiltshire, D., ed., First Australasian Conference on General Relativity and Gravitation, Proceedings of the conference held at the Institute for Theoretical Physics, University of Adelaide, 12 - 17 February 1996, pp. 163-174, (University of Adelaide, Adelaide, Australia, 1996). Related online version (cited on 3 May 2005):

http://arxiv.org/abs/gr-qc/9701056. 4b, 8.3

[337] Sorkin, R.D., "The Statistical Mechanics of Black Hole Thermodynamics", in Wald, R.M., ed., Black Holes and Relativistic Stars, pp. 177-194, (University of Chicago Press, Chicago, U.S.A., 1998). Related online version (cited on 4 May 2005):

http://arxiv.org/abs/gr-qc/9705006. 8 
[338] Sorkin, R.D., and Sudarsky, D., "Large fluctuations in the horizon area and what they can tell us about entropy and quantum gravity", Class. Quantum Grav., 16, 3835-3857, (1999). Related online version (cited on 31 March 2003):

http://arXiv.org/abs/gr-qc/9902051. 4b, 8.3

[339] Starobinsky, A.A., "A new type of isotropic cosmological models without singularity", Phys. Lett. B, 91, 99-102, (1980). 4d, 6.3, 6.5.3, 7.4

[340] Starobinsky, A.A., "Evolution of small excitation of isotropic cosmological models with one loop quantum gravitational corrections", Zh. Eksp. Teor. Fiz., 34, 460-463, (1981). English translation: JETP Lett. 34 (1981) 438. 6.3

[341] Strominger, A., and Trivedi, S.P., "Information consumption by Reissner-Nordstrom black holes", Phys. Rev. D, 48, 5778-5783, (1993). Related online version (cited on 22 January 2008):

http://arXiv.org/abs/hep-th/9302080. 8.3.2

[342] Strominger, A., and Vafa, G., "Microscopic origin of the Bekenstein-Hawking entropy", Phys. Lett. B, 379, 99-104, (1996). Related online version (cited on 31 March 2003):

http://arXiv.org/abs/hep-th/9601029. 8

[343] Su, Z., Chen, L., Yu, X., and Chou, K., "Influence functional, closed time path Green's function and quasidistribution function", Phys. Rev. B, 37, 9810-9812, (1988). 1, 1(b)iii, 4, 8.2.4

[344] Suen, W.-M., "Minkowski space-time is unstable in semiclassical gravity", Phys. Rev. Lett., 62, 2217-2220, (1989). 6.4.1

[345] Suen, W.-M., "Stability of the semiclassical Einstein equation", Phys. Rev. D, 40, 315-326, (1989). 6.4.1

[346] Susskind, L., and Uglum, J., "Black hole entropy in canonical quantum gravity and superstring theory", Phys. Rev. D, 50, 2700-2711, (1994). Related online version (cited on 31 March 2003):

http://arXiv.org/abs/hep-th/9401070. 8

[347] Tichy, W., and Flanagan, É.É., "How unique is the expected stress-energy tensor of a massive scalar field?", Phys. Rev. D, 58, 124007, 1-18, (1998). Related online version (cited on 31 March 2003):

http://arXiv.org/abs/gr-qc/9807015. 6.3

[348] Tomboulis, E., "1/N expansion and renormalization in quantum gravity", Phys. Lett. B, 70, 361-364, (1977). 3.1, 3.2, 3.3.1, 6.4.3, 6.5.2

[349] Trivedi, S.P., "Semiclassical extremal black holes", Phys. Rev. D, 47, 4233-4238, (1993). Related online version (cited on 22 January 2008):

http://arXiv.org/abs/hep-th/9211011. 8.3.2

[350] Twamley, J., "Phase space decoherence: A comparison between consistent histories and environment induced superselection", Phys. Rev. D, 48, 5730-5745, (1993). Related online version (cited on 31 March 2003):

http://arXiv.org/abs/gr-qc/9306004. 1(c)iii, 2.1

[351] Unruh, W.G., "Experimental black hole evaporation", Phys. Rev. Lett., 46, 1351-1353, (1981). 8 
[352] Unruh, W.G., and Zurek, W.H., "Reduction of the wave packet in quantum Brownian motion", Phys. Rev. D, 40, 1071-1094, (1989). 1(c)iii, 2.1, 4

[353] Urakawa, Y., and Maeda, K., "Cosmological Density Fluctuations in Stochastic Gravity: Formalism and Linear Analysis", (2007). URL (cited on 22 January 2008):

http://arXiv.org/abs/arXiv:0710.5342. 7, 7.4

[354] Urakawa, Y., and Maeda, K., "One-loop Corrections to Scalar and Tensor Perturbations during Inflation in Stochastic Gravity", (2008). URL (cited on 22 January 2008):

http://arXiv.org/abs/arXiv:0801.0126. 7.4

[355] Vilenkin, A., "Classical and quantum cosmology of the Starobinsky inflationary model", Phys. Rev. D, 32, 2511-2512, (1985). 4d, 6.3, 6.5.3, 7.4

[356] Volovik, G.E., The Universe in a Helium Droplet, International Series of Monographs on Physics, vol. 117, (Oxford University Press, Oxford, U.K., New York, U.S.A., 2003). 6a, 8

[357] Volovik, G.E., "Fermi-point scenario for emergent gravity", (2007). URL (cited on 22 January 2008):

http://arXiv.org/abs/arXiv:0709.1258. To be published in proceedings of workshop "From Quantum To Emergent Gravity: Theory And Phenomenology", Trieste, Italy, June $11-15,2007.6 \mathrm{a}, 8$

[358] Wald, R.M., "On particle creation by black holes", Commun. Math. Phys., 45, 9-34, (1975). $2,5,8$

[359] Wald, R.M., "The backreaction effect in particle creation in curved spacetime", Commun. Math. Phys., 54, 1-19, (1977). 2, 2.1, 3.1, 4.3.1, 6.1

[360] Wald, R.M., "Trace anomaly of a conformally invariant quantum field in curved space-time", Phys. Rev. D, 17, 1477-1484, (1978). 2, 2.1, 5, 5.2.3

[361] Wald, R.M., General Relativity, (University of Chicago Press, Chicago, U.S.A., 1984). 1a, 1, 4.1

[362] Wald, R.M., Quantum Field Theory in Curved Spacetime and Black Hole Thermodynamics, Chicago Lectures in Physics, (University of Chicago Press, Chicago, U.S.A., 1994). 1b, 2, $3.1,3.1,8.3$

[363] Wald, R.M., "The Thermodynamics of Black Holes", Living Rev. Relativity, 4, lrr-2001-6, (2001). URL (cited on 31 March 2003):

http://www. livingreviews . org/lrr-2001-6. 8

[364] Wald, R.M., "The Thermodynamics of Black Holes", in Bergman, P., and De Sabbata, V., eds., Advances in the Interplay Between Quantum and Gravity Physics, NATO Science Series II, vol. 60, pp. 523-544, (Kluwer, Dordrecht, Netherlands; Boston, U.S.A., 2002). 8

[365] Weber, J., "Fluctuation dissipation theorem", Phys. Rev., 101, 1620-1626, (1956). 8.1.2

[366] Weinberg, S., The Quantum Theory of Fields, Vol. 1: Foundations, (Cambridge University Press, Cambridge, U.K.; New York, U.S.A., 1995). 4.1

[367] Weinberg, S., The Quantum Theory of Fields, Vol. 2: Modern Applications, (Cambridge University Press, Cambridge, U.K.; New York, U.S.A., 1996). 4.1 
[368] Weinberg, S., "Quantum contributions to cosmological correlations", Phys. Rev. D, 72, 043514, (2005). Related online version (cited on 22 January 2008):

http://arXiv.org/abs/hep-th/0506236. 7.4

[369] Weinberg, S., "Quantum contributions to cosmological correlations. II: Can these corrections become large?", Phys. Rev. D, 74, 023508, (2006). Related online version (cited on 22 January 2008):

http://arXiv.org/abs/hep-th/0605244. 7.4

[370] Weiss, U., Quantum Dissipative Systems, Series in Modern Condensed Matter Physics, vol. 2, (World Scientific, Singapore; River Edge, U.S.A., 1993). 1, 1(c)i

[371] Weldon, H.A., "Covariant calculations at finite temperature: The relativistic plasma", Phys. Rev. D, 26, 1394-1407, (1982). 8.2.3

[372] Wen, X.-G., Quantum Field Theory of Many-Body Systems: From the Origin of Sound to an Origin of Light and Electron, (Oxford University Press, Oxford, U.K.; New York, U.S.A., 2004). 8

[373] Wen, X.-G., "An introduction to quantum order, string-net condensation, and emergence of light and fermions", Ann. Phys. (N.Y.), 316, 1-29, (2005). Related online version (cited on 22 January 2008):

http://arXiv.org/abs/cond-mat/0406441. 8

[374] Whelan, J.T., "Modelling the decoherence of spacetime", Phys. Rev. D, 57, 768-797, (1998). Related online version (cited on 31 March 2003):

http://arXiv.org/abs/gr-qc/9612028. 3.2, 4

[375] Wilczek, F., "Quantum purity at a small price: Easing a black hole paradox", in Kalara, S., and Nanopoulos, D.V., eds., Black Holes, Membranes, Wormholes and Superstrings, Proceedings of the International Symposium, Houston Advanced Research Center, USA, 16 - 18 January 1992, (World Scientific, Singapore; River Edge, U.S.A., 1993). Related online version (cited on 22 January 2008):

http://arXiv.org/abs/hep-th/9302096. 8

[376] Witten, E., "On string theory and black holes", Phys. Rev. D, 44, 314-324, (1991). 8

[377] Wu, C.-H., and Ford, L.H., "Fluctuations of the Hawking flux", Phys. Rev. D, 60, 104013, 1-14, (1999). Related online version (cited on 31 March 2003):

http://arXiv.org/abs/gr-qc/9905012. 4b, 8.3, 8.3.2, 8.3.2, 8.3.2, 8.3.2, 8.4

[378] Wu, C.-H., and Ford, L.H., "Quantum fluctuations of radiation pressure", Phys. Rev. D, 64, 045010, 1-12, (2001). Related online version (cited on 31 March 2003):

http://arXiv.org/abs/quant-ph/0012144. 5

[379] Yamaguchi, M., and Yokoyama, J., "Numerical approach to the onset of the electroweak phase transition", Phys. Rev. D, 56, 4544-4561, (1997). Related online version (cited on 31 March 2003):

http://arXiv.org/abs/hep-ph/9707502. 4.2

[380] York Jr, J.W., "Dynamical origin of black-hole radiance", Phys. Rev. D, 28(12), 2929-2945, (1983). 4, 8, 8.2, 8.2.1, 8.2.1

[381] York Jr, J.W., "Black hole in thermal equilibrium with a scalar field: The back-reaction", Phys. Rev. D, 31, 775-784, (1985). 4, 8, 8.2, 8.2.1, 8.2.1, 8.3.1 
[382] York Jr, J.W., "Black hole thermodynamics and the Euclidean Einstein action", Phys. Rev. D, 33, 2092-2099, (1986). 4, 8, 8.2, 8.2.1, 8.2.1

[383] York Jr, J.W., and Schmekel, B.S., "Path integral over black hole fluctuations", Phys. Rev. D, 72, 024022, (2005). Related online version (cited on 22 January 2008): http://arXiv.org/abs/hep-th/0505125. 8.3.2

[384] Yu, Hong-wei, and Ford, L.H., "Lightcone fluctuations in flat spacetimes with nontrivial topology", Phys. Rev. D, 60, 084023, (1999). Related online version (cited on 22 January 2008):

http://arXiv.org/abs/gr-qc/9904082. 2.1, 3.3

[385] Yu, Hong-wei, and Ford, L.H., "Lightcone fluctuations in quantum gravity and extra dimensions", Phys. Lett. B, 496, 107-112, (2000). Related online version (cited on 22 January 2008):

http://arXiv.org/abs/gr-qc/9907037. 2.1, 3.3

[386] Zel'dovich, Y.B., "Particle production in cosmology", Pis. Zh. Eksp. Teor. Fiz., 12, 443-447, (1970). English translation: JETP Lett. 12 (1970) 307-311. 2

[387] Zel'dovich, Y.B., and Starobinsky, A.A., "Particle Production and Vacuum Polarization in an Anisotropic Gravitational Field", Zh. Eksp. Teor. Fiz., 61, 2161-2175, (1971). English translation: Sov. Phys. JETP 34 (1971) 1159-1166. 2

[388] Zemanian, A.H., Distribution Theory and Transform Analysis: An Introduction to Generalized Functions, with Applications, (Dover, New York, U.S.A., 1987). Reprint, slightly corrected, Originally published: New York, McGraw-Hill, 1965. 6.4.2

[389] Zurek, W.H., "Pointer basis of quantum apparatus: into what mixture does the wave packet collapse?", Phys. Rev. D, 24, 1516-1525, (1981). 1(c)iii, 2.1, 4

[390] Zurek, W.H., "Environment induced superselection rules", Phys. Rev. D, 26, 1862-1880, (1982). 1(c)iii, 2.1, 4

[391] Zurek, W.H., "Reduction of the wave packet: How long does it take?", in Moore, G.T., and Scully, M.O., eds., Frontiers of Nonequilibrium Statistical Physics, Proceedings of a NATO Advanced Study Institute, held June 3-16, 1984, in Santa Fe, New Mexico, NATO Science Series B, vol. 135, pp. 145-149, (Plenum Press, New York, U.S.A., 1986). 1(c)iii, 2.1, 4

[392] Zurek, W.H., "Decoherence and the transition from quantum to classical", Phys. Today, 44, 36-44, (1991). 1(c)iii, 2.1, 4

[393] Zurek, W.H., "Preferred states, predictability, classicality and the environment-induced decoherence", Prog. Theor. Phys., 89, 281-312, (1993). 1(c)iii, 2.1, 4 